University of Tennessee Health Science Center

UTHSC Digital Commons

\title{
$5-2009$
}

\section{The Use of Spectroscopic Techniques in the Characterization of Mycobacterial Metabolites}

\author{
Engy Abdelhameed Mahrous \\ University of Tennessee Health Science Center
}

Follow this and additional works at: https://dc.uthsc.edu/dissertations

Part of the Investigative Techniques Commons, Medicinal and Pharmaceutical Chemistry Commons, and the Other Pharmacy and Pharmaceutical Sciences Commons

\section{Recommended Citation}

Mahrous, Engy Abdelhameed, "The Use of Spectroscopic Techniques in the Characterization of Mycobacterial Metabolites" (2009). Theses and Dissertations (ETD). Paper 168. http://dx.doi.org/ 10.21007/etd.cghs.2009.0194.

This Dissertation is brought to you for free and open access by the College of Graduate Health Sciences at UTHSC Digital Commons. It has been accepted for inclusion in Theses and Dissertations (ETD) by an authorized administrator of UTHSC Digital Commons. For more information, please contact jwelch30@uthsc.edu. 


\title{
The Use of Spectroscopic Techniques in the Characterization of Mycobacterial Metabolites
}

\begin{abstract}
The mycobacterial cell wall metabolites have always imposed great challenges to researchers due to their unusual complexity and structural diversity. A lot of research efforts have been directed towards the evaluation of these metabolites and the role they play in the pathogenesis and virulence of different serious human pathogens including Mycobacterium tuberculosis the causative agent of tuberculosis (TB). In the genomic era, it is crucial to develop new methodologies to analyze these components from a global perspective in a comprehensive and well-validated manner.

Towards this end, we developed a rapid NMR-based approach to produce metabolic profiles in the form of $2 \mathrm{D}^{1} \mathrm{H}^{-13} \mathrm{C}$ HSQC maps. Due to NMR low sensitivity, we used ${ }^{13} \mathrm{C}$-isotope enrichment strategy to improve the detection threshold of NMR. We developed and investigated the use of three NMR-based profiles. The first is for the cell wall associated lipids, the second is for covalently bound mycolic acids and the third deals with the complex cell wall polysaccharides. Key structural features and major lipid species were readily identifiable using this technique. This approach can be used for observing changes in the cell wall due to drug treatment, gene mutation and changes in the physiological environment, species characterization and screening for virulence factor expression. It also allows for rapid comparative analysis of several cell wall metabolites within the same sample.

We have used this approach to evaluate the structural diversity displayed in the cell wall metabolites among different Mycobacteria from different clades. We have successfully used the same technique to monitor changes in the carbohydrate and lipid pools from Mycobacteria grown under laboratory conditions that simulate latency and resuscitation from latency. Moreover, we were also able to use such technique to screen for virulence factor in severalM. tuberculosis clinical isolates.
\end{abstract}

Although we have successfully developed and used an NMR approach for metabolite analysis, NMR however remains less sensitive than other spectroscopic techniques like mass spectroscopy (MS). The high sensitivity of MS makes it one of the best candidates for metabolomic studies. However in case of cellular lipids, the low polarity and the variable ionization potential deter its routine use for lipid analysis. Certain metabolites that have low cellular abundance and good ionizability are better analyzed through an MS-based approach. Mycolactones of $M$. ulcerans and other closely related species are good examples for such metabolites and in this work we have demonstrated the utility of MS as a tool for screening for mycolactones from both bacterial culture and tissue samples.

Metabolomics is currently a rapidly growing field and many researchers have directed their attention in developing the right research tools to explore it. Spectroscopic methods along with chromatographic methods have been in the front of this vigorous research effort. The work presented here represents an innovative attempt to utilize such

techniques to investigate the metabolome of some of the most serious human pathogens and discusses the potential area where the application of these techniques can be the most useful.

\section{Document Type}

Dissertation

\section{Degree Name}

Doctor of Philosophy (PhD) 


\section{Program}

Pharmaceutical Sciences

Research Advisor

Richard E. Lee, Ph. D.

\section{Keywords}

Lipidomics, Mass spectroscopy, Mycobacteria cell wall, Mycolactone, NMR spectroscopy, Tuberculosis

\section{Subject Categories}

Analytical, Diagnostic and Therapeutic Techniques and Equipment | Investigative Techniques | Medicinal and Pharmaceutical Chemistry | Medicine and Health Sciences | Other Pharmacy and Pharmaceutical Sciences | Pharmacy and Pharmaceutical Sciences 
THE USE OF SPECTROSCOPIC TECHNIQUES IN THE CHARACTERIZATION OF MYCOBACTERIAL METABOLITES

\author{
A Dissertation \\ Presented for \\ The Graduate Studies Council \\ The University of Tennessee \\ Health Science Center \\ In Partial Fulfillment \\ Of the Requirements for the Degree \\ Doctor of Philosophy \\ From The University of Tennessee
}

By

Engy Abdelhameed Mahrous

May 2009 
Portions of Chapter 2 C 2009 by Humana Press.

Portions of Chapters 3 and 6 (C) 2008

by The American Society for Biochemistry and Molecular Biology.

Parts of Chapter 5 (C) 2006 by The American Society for Microbiology.

All other materials (C) 2009 by Engy Abdelhameed Mahrous.

All rights reserved. 


\section{DEDICATION}

To my family

For all their love and all their sacrifices 


\section{ACKNOWLEDGEMENTS}

I would like to express my sincere gratitude to my advisor Dr. Richard E. Lee for the great opportunity he offered me when he accepted me as a member of his research group. Through his advice, guidance and support, I was able to improve my scientific thinking and research skills. I would like to thank all my committee members, Drs Sarka Beranova, Wei Li, Phillip D. Rogers, Pamela L.C. Small and Jie Zheng who have devoted some of their valuable time to offer me guidance and constructive criticism through the past three years. I would like to offer special thanks to Dr. Pamela L.C. Small who put her confidence in myself and included me in wonderful research experiences. Parts of this work were only possible through her support and cooperation in providing several laboratory strains. Other contributions were made to this work through the generous gifts from Dr. Gurdyal S. Besra at the University of Birmingahm, UK and Dr. Clifton E. Barry III at the NIAID and I am very grateful for them for their support. I am also thankful for Dr. Wei Li for his help in maintaining the departmental NMR spectrometer that I used quite often in this research and I am grateful for all other users for their patience with me during my long experiments. This work was also possible because of funding from the NIH through a grant to Dr. Richard E. Lee.

I would like to extend this acknowledgments to all who helped me in this long learning process that started back in Egypt more than eight years ago; my colleagues in the pharmacognosy department in the college of pharmacy Cairo University, Dr. Malak Kotb and her research group in the VA Medical center in Memphis and all faculty and colleagues in the medicinal chemistry department at the University of Tennessee, HSC. I am fully indebted to Robin E. Lee who worked very closely with me in the past five years and whose advice and discussion helped shape this work as well as my research skills. I greatly appreciate the time she devoted for my training despite her other responsibilities. She has also contributed to this work by doing all the microbiology work for strains that require handling in biosafety level-3 facility.

On a personal level, I owe everything I have achieved to my wonderful parents who supported my goal to pursue my career in the United States even when it meant that I will be thousands of miles away when they need my help. I hope they feel satisfaction that their sacrifices and prayers are paid off. I also appreciate the moral support I had from my brother, sisters and their families in Egypt. Their e-mails and phone conversations have kept me motivated. I fall short of words to describe my appreciation to my husband Dr. Mohmmed M. Nooh who has been my unwavering supporter during this journey and has always believed in me when I did not believe in myself. I am also thankful for him for the weekends and evenings that he has to watch over our children. To my children Malak and Omar, I am always grateful for your smiles and giggles. These are always my ultimate refuge in the face of frustration.

I have been also blessed with many wonderful friendships in Memphis, too many to be counted here. I am especially thankful for members of Memphis Muslim community who have surrounded me and my family with their love and care in the past five years. 


\begin{abstract}
The mycobacterial cell wall metabolites have always imposed great challenges to researchers due to their unusual complexity and structural diversity. A lot of research efforts have been directed towards the evaluation of these metabolites and the role they play in the pathogenesis and virulence of different serious human pathogens including Mycobacterium tuberculosis the causative agent of tuberculosis (TB). In the genomic era, it is crucial to develop new methodologies to analyze these components from a global perspective in a comprehensive and well-validated manner.
\end{abstract}

Towards this end, we developed a rapid NMR-based approach to produce metabolic profiles in the form of $2 \mathrm{D}^{1} \mathrm{H}_{-}{ }^{13} \mathrm{C}$ HSQC maps. Due to NMR low sensitivity, we used ${ }^{13} \mathrm{C}$-isotope enrichment strategy to improve the detection threshold of NMR. We developed and investigated the use of three NMR-based profiles. The first is for the cell wall associated lipids, the second is for covalently bound mycolic acids and the third deals with the complex cell wall polysaccharides. Key structural features and major lipid species were readily identifiable using this technique. This approach can be used for observing changes in the cell wall due to drug treatment, gene mutation and changes in the physiological environment, species characterization and screening for virulence factor expression. It also allows for rapid comparative analysis of several cell wall metabolites within the same sample.

We have used this approach to evaluate the structural diversity displayed in the cell wall metabolites among different Mycobacteria from different clades. We have successfully used the same technique to monitor changes in the carbohydrate and lipid pools from Mycobacteria grown under laboratory conditions that simulate latency and resuscitation from latency. Moreover, we were also able to use such technique to screen for virulence factor in several M. tuberculosis clinical isolates.

Although we have successfully developed and used an NMR approach for metabolite analysis, NMR however remains less sensitive than other spectroscopic techniques like mass spectrometry(MS). The high sensitivity of MS makes it one of the best candidates for metabolomic studies. However in case of cellular lipids, the low polarity and the variable ionization potential deter its routine use for lipid analysis. Certain metabolites that have low cellular abundance and good ionizability are better analyzed through an MS-based approach. Mycolactones of M. ulcerans and other closely related species are good examples for such metabolites and in this work we have demonstrated the utility of MS as a tool for screening for mycolactones from both bacterial culture and tissue samples.

Metabolomics is currently a rapidly growing field and many researchers have directed their attention in developing the right research tools to explore it. Spectroscopic methods along with chromatographic methods have been in the front of this vigorous research effort. The work presented here represents an innovative attempt to utilize such 
techniques to investigate the metabolome of some of the most serious human pathogens and discusses the potential area where the application of these techniques can be the most useful. 


\section{TABLE OF CONTENTS}

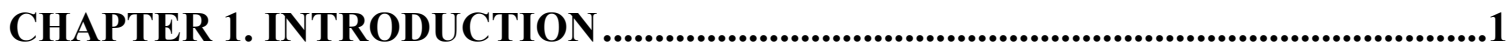

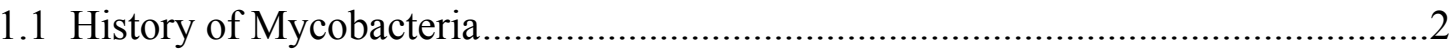

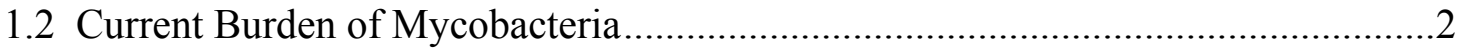

1.3 The Pathogenesis of Mycobacterium tuberculosis ................................................

1.4 Mycobacteria Cell Envelope ………………………........................................

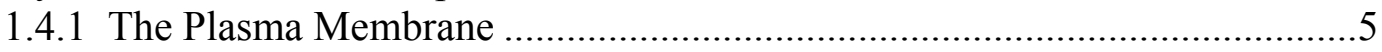

1.4.2 The Peptidoglycan Mycolyl Arabinogalactan Complex (PG-mAG) .............

1.4.3 Extractable Cell Wall Lipids...................................................................12

1.5 Role of the Mycobacterial Cell Wall in Virulance................................................25

1.6 Mycobacterial Cell Wall as Diagnostic and Taxonomic Tool ..............................26

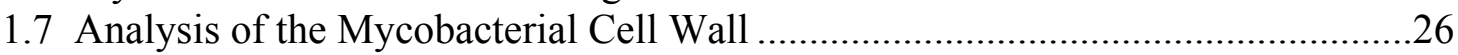

1.7.1 Analysis of Peptidoglycan Structure.......................................................26

1.7.2 Analysis of Arabinogalactan Complex …………..................................2

1.7.3 Free Lipid Extraction .........................................................................28

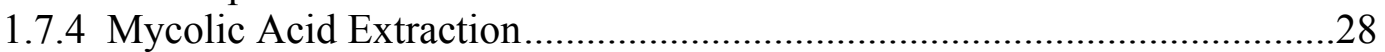

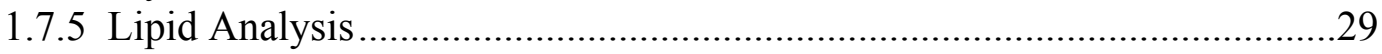

1.8 Current Challenges in Mycobacteria Research …….............................................33

1.8.1 Mycobacteria Latency and Persistence........................................................34

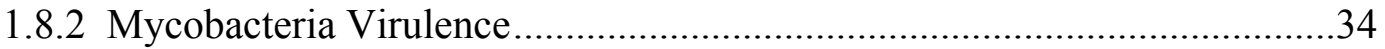

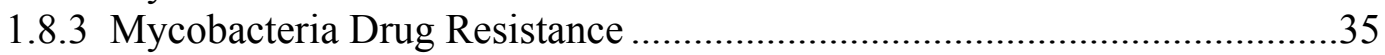

1.8.4 NTM Infections and Emerging Mycobacterial Diseases ...............................35

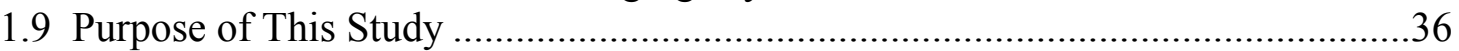

\section{CHAPTER 2. DEVELOPMENT OF A NEW METHOD FOR ANALYSIS OF}

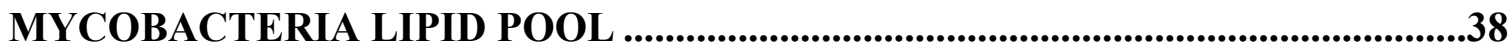

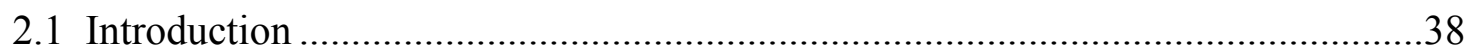

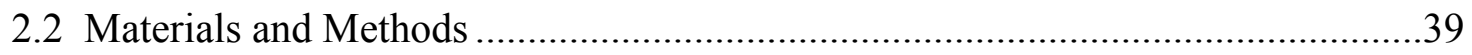

2.2.1 Strains and Growth Conditions ..................................................................39

2.2.2 Total Lipid Extraction and Fractionation..................................................40

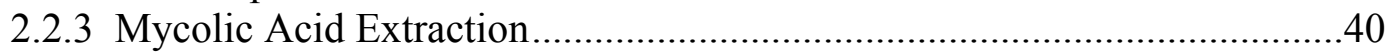

2.2.4 NMR Acquisition and Data Processing .....................................................40

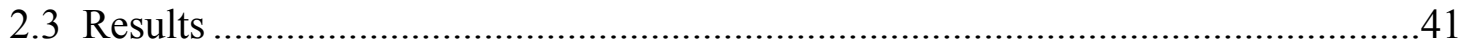

2.3.1 Production of NMR Profiles and Assigning Signals of Some Cell Wall Lipids.

2.3.2 Effect of Different Growth Conditions .........................................................41

2.3.3 Effect of Initial Culture Volume, Solvent and Duration of Extraction.........46

2.3.4 The Use of DMSO as an Internal Standard .............................................49

2.3.5 Reproducibility of the Lipid Profiles .........................................................49

2.3.6 Lipid Fractionation through Solvent Partition .............................................54

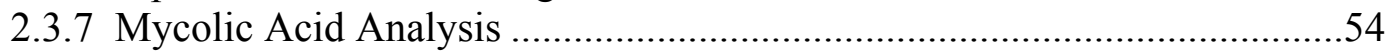

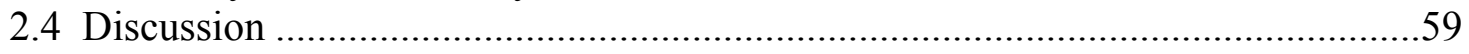

2.4.1 A Summary of a Standard Protocol for Comprehensive Analysis ..............59 
2.4.2 Amenability of the Protocol for Both Comprehensive and Targeted Analysis

2.4.3 Biomarker Assignment through Literature Source and Isolation of

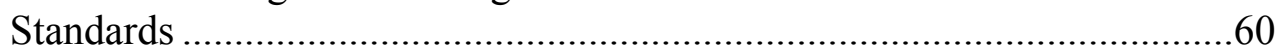

2.5 Conclusion .60

\section{CHAPTER 3. USE OF 2D-HSQC NMR FOR ANALYSIS OF} MYCOBACTERIA CELL WALL ................................................................62

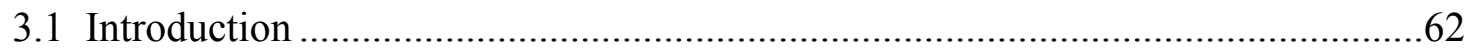

3.1.1 Mycobacteria Human Pathogens .............................................................62

3.1.2 Mycobacteria Animal Pathogens ...........................................................63

3.1.3 Old Classification of Mycobacteria ……………........................................63

3.1.4 Variability in Cellular Lipid among Mycobacteria......................................63

3.1.5 Value of Lipid Profiling of Different Mycobacteria Species........................64

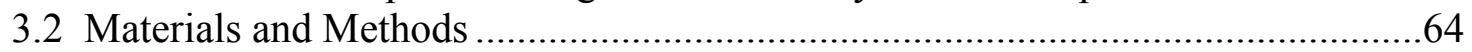

3.2.1 Strains and Growth Conditions …………………...................................64

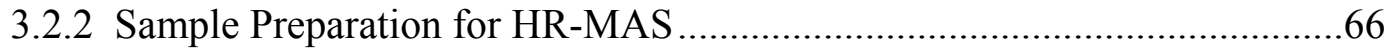

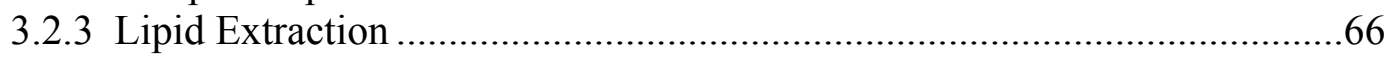

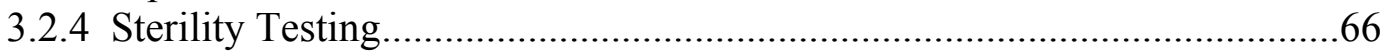

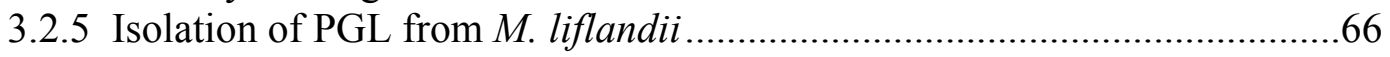

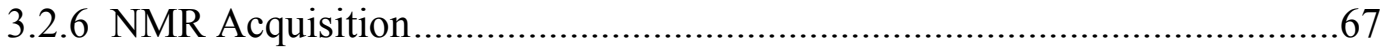

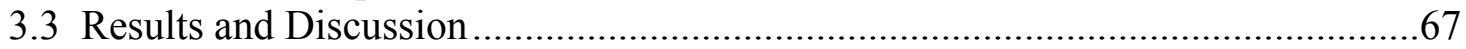

3.3.1 Characteristic Features of Some Species .....................................................67

3.3.2 Comparison between Slow Growers and Fast Growers ………………........69

3.3.3 Comparison between Intracellular and Extracellular Pathogens ...................71

3.3.4 Evidence of Evolutionary Relationship between M. liflandii and $M$.

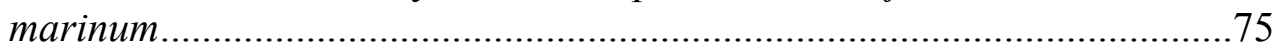

3.3.5 Different Lipid Profiles for Different Laboratory Strains and Clinical

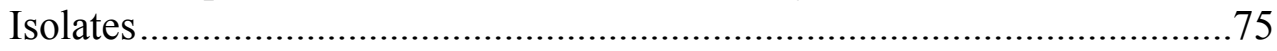

3.3.6 Lipid Profiles of Other Bacteria: Corynebacterium and Bacillus .................77

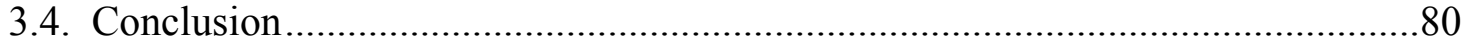

3 4.1 NMR-Based Approach for Comprehensive Cell Wall Analysis ...................80

3.4.2 Species Differentiation Using NMR Metabolic Profiling ............................80

3.4.3 Applicability to Other Bacterial Species......................................................81

\section{CHAPTER 4. METABOLOMIC STUDIES OF MYCOBACTERIA}

ADAPTATION TO STRESS CONDITIONS ...................................................................82

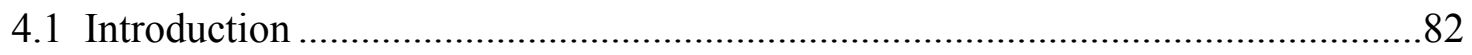

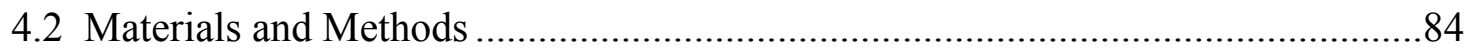

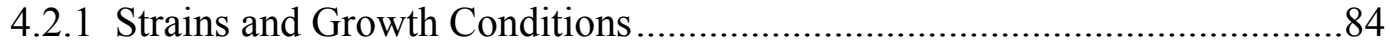

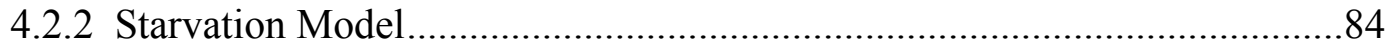

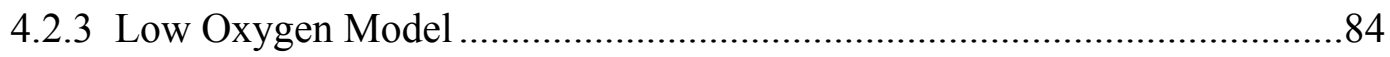

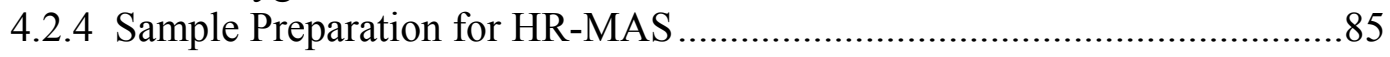

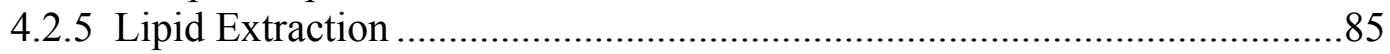

4.2.6 NMR Acquisition and Data Processing ......................................................85 


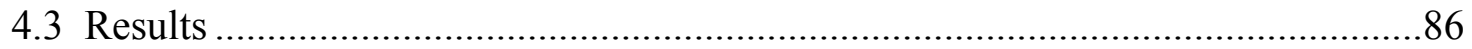

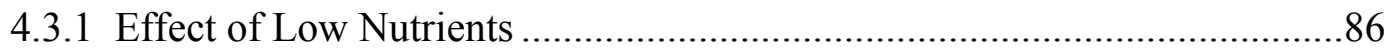

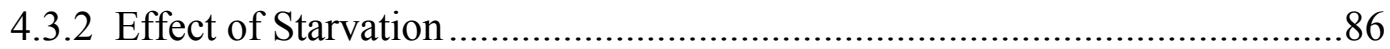

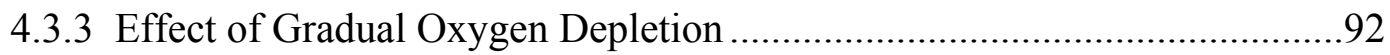

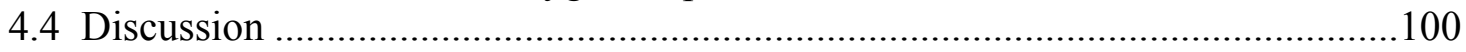

4.4.1 Stability of the Structural Components of the Mycobacteria Cell Wall ....100

4.4.2 Phospholipids as Indicators of Cell Viability .............................................100

4.4.3 Triacyl Glycerols as Favorite Energy Sources..........................................100

4.4.4 Process of Reactivation Has Optimum Goal ..............................................101

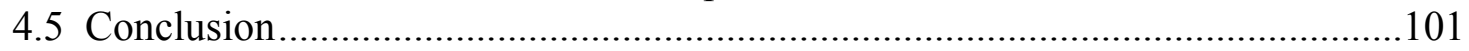

\section{CHAPTER 5. TWO CASE STUDIES FOR THE USE OF BIOANALYTICAL TOOLS IN SCREENING FOR VIRULENCE DETERMINANTS OF \\ PATHOGENIC MYCOBACTERIA ..............................................................103}

5.1 Case Study-I: Screening Various Mycobacteria Species for the Production of

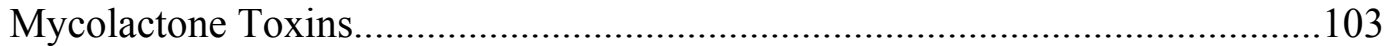

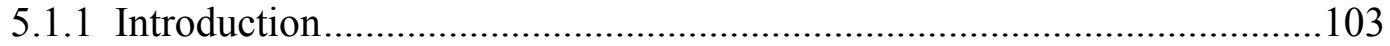

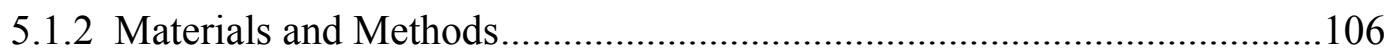

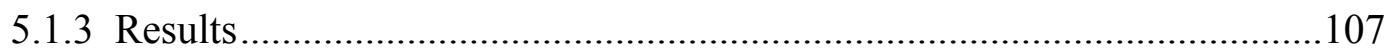

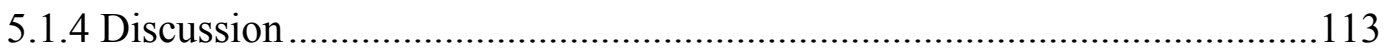

5.2 Case Study II: Screening Mycobacterium tuberculosis Clinical Isolates for

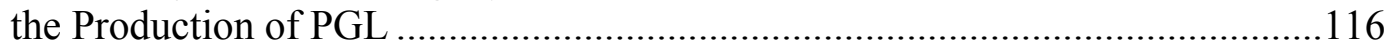

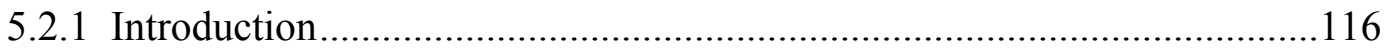

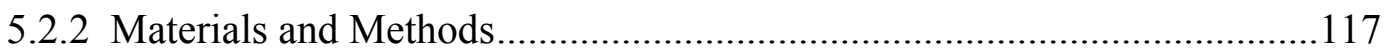

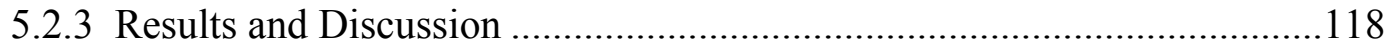

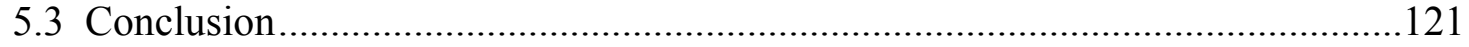

CHAPTER 6. SUMMARY AND CONCLUSION_....................................................126

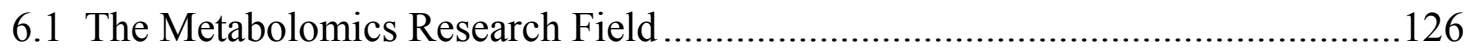

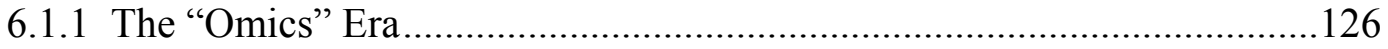

6.1.2 Challenges in Metabolomics.................................................................127

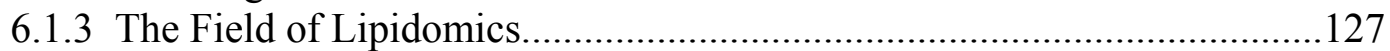

6.1.4 Designing a New Lipidomics Approach...............................................128

6.2 New Techniques in Lipidomics...................................................................128

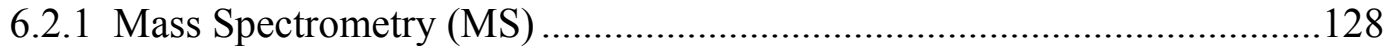

6.2.2 Chromatographic Separation Methods HPLC and GC ..............................129

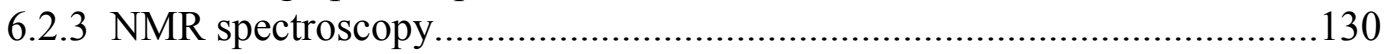

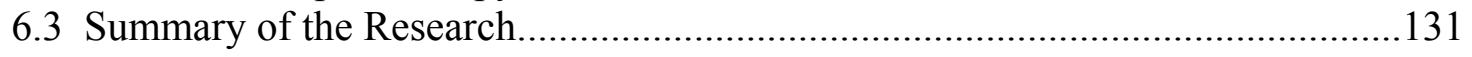

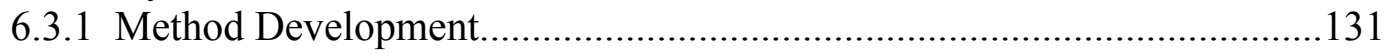

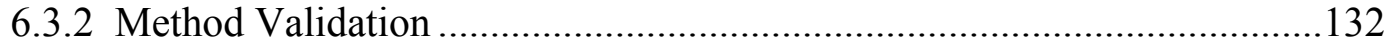

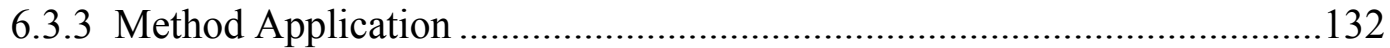

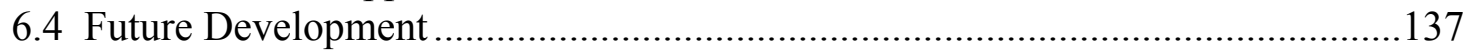

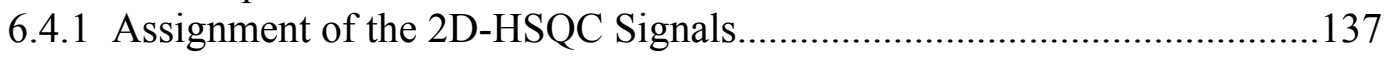

6.4.2 Method Standardization and Data Analysis..............................................138

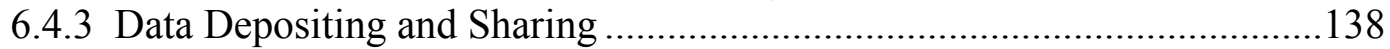

6.5 How Metabolomics May Change Life Science .................................................138 
LIST OF REFERENCES............................................................................................140

APPENDIX A. SUPPLEMENTARY FIGURES FOR CHAPTER 2 .........................168

APPENDIX B. SUPPLEMENTARY FIGURES FOR CHAPTER 3 .........................178

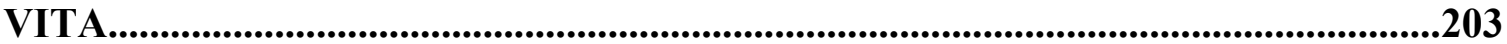




\section{LIST OF TABLES}

Table 2.1 Chemical Shift Values of Major Lipid Biomarkers.................................. 43

Table 2.2 Chemical Shift Values of Major Mycolic Acid Biomarkers ..................... 58

Table 3.1 Summary of the Mycobacteria Species Investigated in This Study ........... 65

Table 5.1 Differences in the Structures of Mycolactones Isolated So Far................. 104

Table 5.2 Mycolactone Ions Identified in Different Mycobacteria Strains ............... 111

Table 5.3 Proton Chemical Shifts of the Major Isomer of Mycolactone F............... 114

Table 5.4 Results from Screening M. tuberculosis Clinical Isolates for PGL

Production ....................................................................................... 122 


\section{LIST OF FIGURES}

Figure 1.1 Schematic Presentation of the Cell Wall of Mycobacteria .......................... 6

Figure 1.2 Phospholipids of the Mycobacterial Plasma Membrane.............................. 6

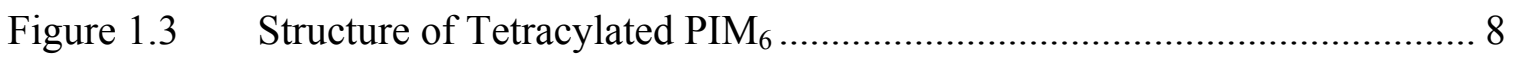

Figure 1.4 Schematic Presentation of the Structure of Mycobacteria AG ................. 10

Figure 1.5 Major Types of Cell Wall Mycolic Acids............................................... 11

Figure 1.6 Schematic Representation of the Cell Wall manLAM ............................. 14

Figure 1.7 Representative Examples of Acylated Trehalose .................................... 15

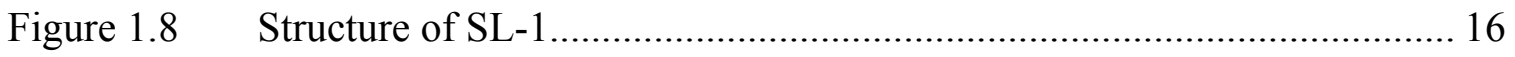

Figure 1.9 Examples of Phthienoic and Mycocerosic Acids..................................... 18

Figure 1.10 Structure of Phthiocerol Derivatives.................................................... 20

Figure 1.11 Structures of Some PGL from Different Mycobacteria............................. 21

Figure 1.12 The Tetrapeptide Core of GPL …………...................................... 22

Figure 1.13 General Structure of Mycobactins ........................................................ 24

Figure 1.14 Structure of Some Examples of Carotenoids ........................................... 24

Figure 1.15 General Structure of Menaquinone ....................................................... 24

Figure 2.1 2D-HSQC Lipid Profile of M. bovis BCG ……....................................... 42

Figure 2.2 Effect of the Composition of Growth Media on the Lipid Profile of $M$.

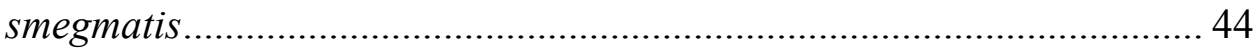

Figure 2.3 Effect of the Use of Different Growth Media on the Lipid Profile of $M$.

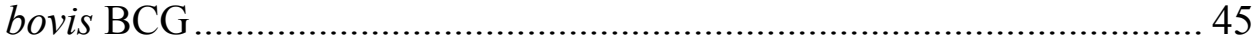

Figure 2.4 Lipid Profile of M. bovis BCG Harvested at Different Growth Stages .... 47

Figure 2.5 Effect of Acetate Labeling on the Lipid Profile of M. marinum .............. 48 
Figure 2.6 2D-HSQC Spectrum Produced by Repeated Extraction of the Cell Pellet

Figure 2.7 Effect of Using Acetone- $\mathrm{d}_{6}$ as the Extracting Solvent............................ 51

Figure 2.8 Calibrating Curve of Different Concentrations of DMSO........................ 52

Figure 2.9 Assessment of the Reproducibility of Data Obtained from 2D-HSQC Lipid Profiles ......................................................................................... 53

Figure 2.10 Fractionation of the Lipid Extract of $M$. bovis BCG ................................ 55

Figure 2.11 Fractionation of the Lipid Extract of M. avium ...................................... 56

Figure 2.12 2D-HSQC Profile of Mycolic Acids Isolated from M. bovis BCG .......... 57

Figure 3.1 Interspecies Variabilities in the Cell Wall LAM as Observed in the HR-MAS Spectra of Different Mycobacteria ............................................. 68

Figure 3.2 Comparison between the HR-MAS and Lipid Profiles of Some Fast Growing and Slow Growing Mycobacteria 70

Figure 3.3 Comparison between the Extracellular M. ulcerans and the Intracellular

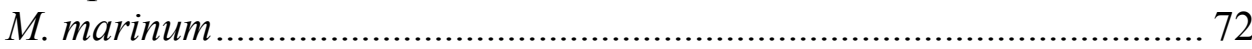

Figure 3.4 Lipidomics Characterization of M. liflandii............................................. 76

Figure 3.5 Comparison between the HR-MAS Profile of $C$. glutamicum and $M$.

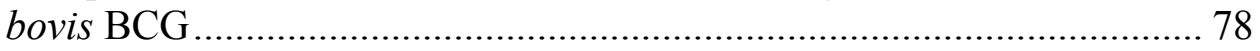

Figure 3.6 2D-HSQC Lipid Profile of C. glutamicum ……………......................... 79

Figure 3.7 2D-HSQC Lipid Profile of Bacillus Species .......................................... 79

Figure 4.1 Effect of Limited Nutrients Supply on the Cellular Lipid Content of M. bovis $\mathrm{BCG}$

Figure 4.2 Effect of Limited Nutrients Supply on the Cell Wall AG and LAM of

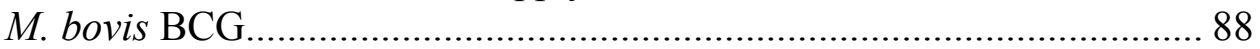

Figure 4.3 Effect of Prolonged Starvation in PBS on the Cell Wall Lipids of $M$.

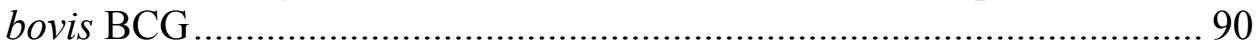

Figure 4.4 Effect of Prolonged Starvation in PBS on the Cell Wall Polysaccharides of $M$. bovis BCG 93 
Figure 4.5 Effect of Gradual Oxygen Depletion on the Cell Wall Lipids of $M$.

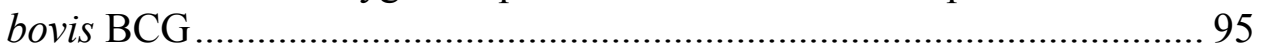

Figure 4.6 Effect of Oxygen Depletion on the Cell Wall Polysaccharides of $M$. bovis BCG........................................................................................... 97

Figure 4.7 Effect of Gradual Oxygen Depletion on the Cell Wall Mycolic Acid of M. bovis BCG...................................................................................... 99

Figure 5.1 General Structure of Mycolactones ..................................................... 104

Figure 5.2 Domain and Module Organization of Mycolactone A/B PKS Genes .... 105

Figure 5.3 Major Fragmentation Pathways of Mycolactone A/B ............................ 108

Figure 5.4 MS Analysis of Different Mycolactone Molecules ................................ 110

Figure 5.5 HPLC Profiles of the ASL Extract from Different Mycobacteria .......... 112

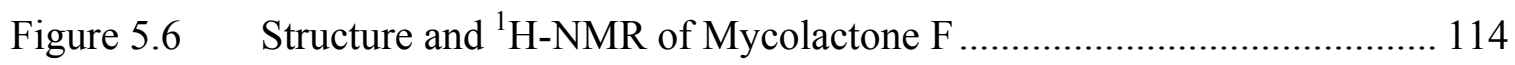

Figure 5.7 Selection of Biomarker Signals of M. tuberculosis PGL........................ 120

Figure 6.1 Effect of Gene Mutation on the Cell Wall of C. glutamicum ................. 134

Figure 6.2 Effect of Ethambutol Treatment in the Cell Wall of M. smegmatis ....... 136 


\section{LIST OF ABBREVIATIONS}

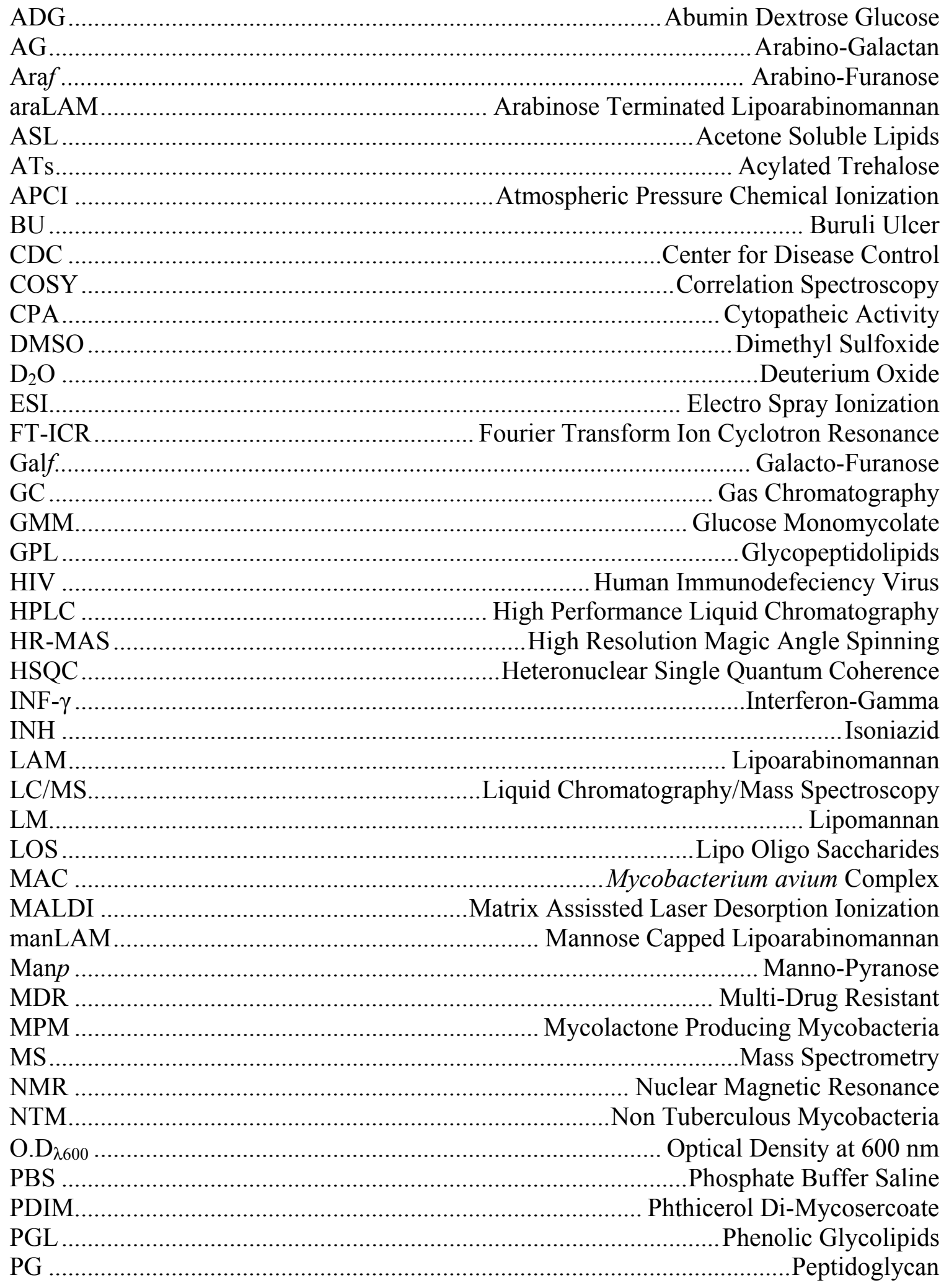


PG-mAG Peptidoglycan-mycolyl Arabinogalactan PiLAM Phosphoinositol Capped Lipoarabinomannan

PIMs Phospho Inositol Mannosides

PKS Polyketide Synthase

PL Phospholipids

PPM .Part Per Million

SL Sulfolipids

TAG Tri Acyl Glycerol

TB Tuberculosis

TLC Thin Layer Chromatography

TDM. Trehalose Dimycolate

TLR-2 Toll-Like Receptor-2

TMM Trehalose Mono Mycolate

TNF- $\alpha$ Tumor Necrosis Factor-Alpha unFA Unsaturated Fatty Acids

WHO World Health Organization

XDR Extensively Drug Resistant 2D-TLC

3D HCCH-TOCSY ... Three Dimensional Proton Carbon Total Correlation Spectroscopy 3D-HCCH COSY. Three Dimensional Proton Carbon Correlation Spectroscopy 


\section{CHAPTER 1. INTRODUCTION}

Mycobacterium, the single genus of the family Mycobacteriaceae, is described as rodshaped Gram positive aerobic to microarophilic bacilli with GC- rich DNA.

Taxonomically, it is placed in the same suborder Corynebacterianeae with Corynbacteria, Nocardia and Rhodoccoci which are all referred to as Actinomycetales. ${ }^{1}$ In general, Mycobacteria are ubiquitous organisms that can be isolated from tap water, house dust and soil but few are clinically important pathogens that are associated with serious health problems. ${ }^{2-5}$ Others are emerging as opportunistic pathogens and are increasingly imposing new health challenges. ${ }^{6}$ For many years, the diagnostic feature that was used to describe Mycobacteria was its "acid-fastness", a phrase that refers to the ability of the stained bacilli to resist decolorization by acidic ethanol when exposed to cationic triphenyl methane dyes such as fuschin. ${ }^{7}$ The chemical nature of this interaction has been widely speculated but with contradicting evidence pointing in different directions, it remains a debatable research point. ${ }^{8-10}$

Historically, several classifications of Mycobacteria have been used and most have relied on a specific morphological feature or a biochemical reaction. The most acceptable nowadays is the traditional classification of Mycobacteria into slow growers and fast growers subgroups ${ }^{1}$ (excluding the uncultivable $M$. leprae). This traditional classification is surprisingly in agreement with modern $16 \mathrm{~S}$ rRNA-based classification. ${ }^{11}$ In addition, most pathogenic Mycobacteria fall in the slow growers subgroup with few exceptions. ${ }^{1}$ Other classic descriptions such as atypical Mycobacteria and chromogenic Mycobacteria have become increasingly outdated and clinically irrelevant as they are biased to a single feature like the frequent association with mammalian diseases or the ability to produce certain pigments. ${ }^{7}$

In the clinic, the most important mycobacterium is that of $M$. tuberculosis, the causative agent of tuberculosis (TB), a disease that consumes around two million lives every year. ${ }^{12}$ Other common mycobacterial human pathogens are $M$. leprae the causative agent of leprosy, M. marinum an intracellular pathogen that causes granulomateous skin infection $^{13}$ and $M$. ulcerans a pathogen responsible for the Buruli ulcer endemic in some tropical regions. ${ }^{14}$ However, with an expanding population of immunocompromised patients, infections with environmental Mycobacteria that do not cause diseases to human under normal conditions have been on the rise. ${ }^{15}$ Diseases caused by these opportunistic Mycobacteria have contributed to the mortality and morbidity of thousands of cases of HIV patients. Such infections happen through unavoidable routine activities and can result in either tuberculosis like pulmonary symptoms, lymphaniditis or more severe systemic mycobacteriosis. ${ }^{16}$ Most common among these infections are infections with Mycobacterium avium complex (MAC) which includes mainly $M$. avium and $M$. intracellulare. ${ }^{12,17}$

Some fast growers, like members of $M$. fortuitum complex, M. abscessus and $M$. chelonae, have also been isolated from the sputum of immunocompromised patients. ${ }^{18}$ Most other fast growers, however, are saprophytic species like M. smegmatis and $M$. 
phlei that rarely cause diseases in humans but are used in the laboratory as surrogates to Mycobacterium tuberculosis which can only be handled in biosafety level-3 facilities to provide maximum protection to researchers and laboratory workers and limit the spread of this infectious agent.

\subsection{History of Mycobacteria}

Mycobacteria pathogens were among the first microorganisms to be discovered as the causative agents of human diseases. Indeed, the first human pathogen to be described was that of Mycobacterium leprae the etiological cause of leprosy when in 1873 Gerhard Hansen reported the presence of rod shaped "infectious substance" in the tissue of infected patients without identifying it as bacteria. ${ }^{19}$ It was not until 1880 that Albert Neisser have stained and identified the bacilli as the etiological cause of leprosy. During the time of Neisser's successful characterization, the work that led to the ground breaking discovery of Mycobacterium tuberculosis as the causative agent of tuberculosis was also taking place. On March 24, 1882, Robert Koch, the father of microbiology, gave one of the most important lectures in medical history to announce his discovery of the causative agent of TB. ${ }^{20}$ In his lecture that evening, Koch illustrated to his audience how he successfully isolated, stained and cultured the tubercle's bacilli. ${ }^{21}$ Koch was awarded the Nobel Prize for "his investigations and discoveries in relation to tuberculosis" in $1905 .{ }^{22}$

But long before Koch made his discovery, Mycobacteria species and their associated diseases have existed throughout human history. Molecular evidence of tuberculosis was found in Egyptian mummies. ${ }^{23,24}$ Chinese, Indian and Egyptian scriptures dated as far back as to 4000B.C contained description of both tuberculosis and leprosy. ${ }^{20} \mathrm{It}$ is estimated that TB alone, also known as consumption or white plague, has consumed at least one billion human lives between 1700 and $1900 .{ }^{20}$ Other mycobacterial diseases have surfaced more recently. The third most common mycobacterial disease, Buruli ulcer, was not defined and its causative agent M. ulcerans has not been described until 1948 despite some initial reporting of an ulcerative skin disease in Uganda by Sir Robert Cook in $1897 .{ }^{25,26}$ With the progress of scientific research tools and the ever-rapid accumulation of human knowledge, the genus mycobacterium now includes nearly 80 species, the majority of them were discovered during the last five decades although most have existed since prehistoric times.

\subsection{Current Burden of Mycobacteria}

The single most important species of the genus mycobacterium is $M$. tuberculosis, a single species that infects one third of the world population and is responsible for nearly two million deaths every year due to tuberculosis (TB). ${ }^{12}$ The World Health Organization (WHO) estimates that M. tuberculosis infects more than one person every second worldwide with the latent form of the disease. Among those infected, $10 \%$ will develop an active infectious form of the disease in their lifetime and can infect 10-15 persons a year if left untreated. ${ }^{27}$ 
Despite the long history of human encounter with TB, the fight against this pathogen has not been won yet especially with constant emergence of new challenges. Of the nine million new cases reported every year, more than $70 \%$ of these cases are reported in Asia and sub-Saharan Africa where the failure to control TB infection is attributed to the HIV/AIDS epidemic in this region. ${ }^{28,29}$ It is estimated that one third of HIV patients are co-infected with TB which is considered the number one killer of HIV patients. ${ }^{30}$ This co-infection also makes it 800 times more likely for an individual to develop an active form of the disease and thus spread the TB infection. ${ }^{30}$ With the devastating human health cost comes also a great economic burden as $80 \%$ of TB patients are between 15 and 49 years of age amidst their productive life and are in most cases the only supporters of their large families. ${ }^{31}$

In the last two decades, we were confronted with another challenge with the spread of multi drug resistant TB (MDR-TB) and more recently extensively drug resistance TB $(\mathrm{XDR}-\mathrm{TB})^{32}$ which has in turn complicated the HIV/TB problem. ${ }^{33,34} \mathrm{MDR}-\mathrm{TB}$ is a form of the disease caused by strains that resist treatment with more than one of the frontline anti-tuberculosis drugs. For example, an MDR-TB strain resistant to both rifampin and isoniazid (INH) was associated with an outbreak in New York City in the early 1990s that grabbed scientists' attention and caused public panic. ${ }^{35}$ XDR-TB is a term introduced in 2006 to describe another form of the disease caused by strains that are resistant to both rifampin and INH in addition to key second line anti-TB treatment including a fluroquinolone and one injectable drug from the aminoglycosides and capreomycin group. ${ }^{34,36}$ Although anti-TB drugs have been in use since the 1940s, the current repertoire of anti-TB drugs has now limited capabilities and low potential to provide a tool for eradicating TB. Many global health initiatives have been launched in the recent years to counteract the spread of this disease and help treat the millions affected by it. Yet, there has been no significant down turn in the global rates of incidence or mortality due to tuberculosis.

On the other hand, the prevalence of two other mycobacterial human diseases (leprosy and Buruli ulcer) stands at about 250,000 cases worldwide with their associated cost of treatment and lost productivity. ${ }^{37,38}$ Being limited in their geographic distribution to the tropical regions, these diseases receive limited attention from both research and health organizations despite the fact that a lot of questions remain regarding disease transmission and the availability of limited treatment options.

It is also worth mentioning that at least thirty Mycobacteria species are known to cause diseases to domestic and wild animals and have significant economic implications. This group includes $M$. bovis, Mycobacterium marinum, $M$. fortuitum, $M$. chelonae and M. avium subsp paratuberculosis among others. M. bovis is the cause of tuberculosis in cows while $M$. avium subsp. paratuberculosis is believed to be the causative agents of Johne's disease in cattle and some evidence points to a role of this organism in human Crohn's disease, a painful chronic disease that affect 500,000 in the US alone..$^{39,40}$ Systemic mycobacteriosis is considered a major problem for aquatic animals. A number of Mycobacteria that are natural habitat of water are known to cause systemic infections in fish. ${ }^{41}$ Recently, a new species, M. pseudoshotsii, was identified as the cause of an 
endemic mycobacteriosis in the sea striped bass in Chesapeake Bay. ${ }^{42,43}$ So, in addition to the negative human health impact of Mycobacteria, there are both economic and environmental burdens that threaten our aquatic and agricultural reserves.

\subsection{The Pathogenesis of Mycobacterium tuberculosis}

Beside the fact that Mycobacterium tuberculosis is the main cause of pulmonary tuberculosis, it is also responsible for another serious form of tuberculosis, disseminated or extra pulmonary tuberculosis, in neonates and severely immune-compromised patients. ${ }^{44,45}$ In the disseminated form of TB, the bacilli can escape the lungs and disseminate to other organs through the lymph system most commonly bones and joints. ${ }^{46}$ Both the pulmonary and the extra-pulmonary diseases spread through inhalation of the bacterium. A host can inhale contaminated droplets produced through the coughing and sneezing of an infected individual who spreads a load of Mycobacteria into the atmosphere where the bacilli can survive for several hours. ${ }^{47}$ It is estimated that the infectious dose to the new host can be as low as a single bacterium.

Once inhaled, the bacilli travel deep into the airways where they are engulfed by alveolar macrophages in an attempt to destroy the invading pathogen. ${ }^{48,49}$ However, the TB bacilli have the ability to survive and replicate inside these macrophages by inhibiting maturation and acidification the phagosome. ${ }^{50}$ At this stage, multiple cytokines are released by the infected macrophages (TNF- $\alpha$ is the most important). This cytokine cascade recruits successive waves of $\mathrm{CD} 4^{+}, \mathrm{CD} 8^{+}$and natural killers $\mathrm{T}$-cells to contain the infection. As these cells start to surround the infected macrophages, they initiate the formation of a well organized pulmonary lesion (known as tubercle or granuloma) the pathological signature of tuberculosis. ${ }^{49}$ Once the infected macrophages are surrounded with T-cells, the immune response to the infection is suppressed through the secretion of INF- $\gamma$ from these cells. With time progression, the peripheries of these tubercles become surrounded by fibrous cuff and the blood supply to the lesion diminishes. ${ }^{51}$ The bacilli at the core of these lesions can remain viable for extended period of time (dormant bacilli) but in a contained phase where the host cannot transmit the infection to others (latent infection) ${ }^{51,52}$ Reports indicate that one third of the world population are infected with this latent form of the disease and that between $5-10 \%$ of these individuals will develop an active form of the disease later on their life time. ${ }^{52,53}$

The activation of a latent infection is usually a result of a deteriorating host immune system that can be related to malnutrition, co-infection with HIV, immunosuppressive therapy or other factors. For example, when the $\mathrm{CD}^{+}$cell count diminishes as a result of HIV infection, the granuloma can no longer be contained and its walls rupture allowing the migration of the free bacilli to the airways. At this point, the acid fast bacilli start to appear in the patient sputum and the host becomes infective to those who are in close contact. ${ }^{49}$ Usually six to eight weeks of anti-tuberculosis therapy (rifampin, INH, pyrazinamide and ethambutol or RIPE) are necessary to clear the sputum but four more months of therapy (rifampin and INH) are still required to eliminate all loci of infectious bacteria with all different populations to deter the development of resistance. ${ }^{54-56}$ 


\subsection{Mycobacteria Cell Envelope}

The persistence of TB as a major health problem is attributed to the ability of Mycobacteria to adapt to different environmental conditions inside and outside the human body. ${ }^{57}$ Not only can $M$. tuberculosis survive and resist the killing mechanism inside human macrophages, but it also has the capacity to slow down its metabolism and replication to stay hidden from the host immune system for extended periods of time. Still at this latent phase, Mycobacteria retain the ability to monitor the host immune system and remain in a state of preparedness to be activated once host defense mechanisms are compromised. ${ }^{53}$

For a better understanding of Mycobacteria pathogenicity, it is crucial to examine its cell wall as it accounts for $60 \%$ of the bacterial cell weight and is commonly believed to be partially responsible for Mycobacteria drug resistance and virulence. Like all eubacteria, the Mycobacteria cell is surrounded by a peptidoglycan-based cell wall, but a unique and very complex cell wall assures its survival and resistance to different killing mechanisms by the host immune system (Figure 1.1). The cell wall of Mycobacteria (and in fact all actinomycetales) is classified on a chemical basis as chemotype IV cell wall. ${ }^{58}$ In addition to the plasma membrane and the peptidoglycan, chemotype IV is characterized by the presence of a special polysaccharide structure known as arabinogalactan (AG). This arabinogalactan is terminally substituted with long chain fatty acids that vary in length based on the bacterial species and tend to form an outer lipid layer that function as a permeability barrier. ${ }^{59,60}$ The total composition of the cell wall will be discussed in more details below.

\subsubsection{The Plasma Membrane}

\subsubsection{Membrane general features}

The plasma membrane of Mycobacteria has all the general features recognized in the membranes of other prokaryotes. It has the general arrangement of a phospholipids bilayer. The phospholipids of the plasma membrane are mainly derivatives of phosphatidic acids $^{59}$ (Figure 1.2). The most abundant of which is bis-phosphatidyl glycerol, also known as cardiolipin. ${ }^{61}$ Other phospholipids include phosphatidyl ethanolamine, phosphatidyl inositol and its corresponding mannosides PIMs, which are located mainly in the membrane outer leaflets. The fatty acid substitutions in all membrane phospholipids are mainly palmitic (C-16), hexadecenoic (unsaturated $\mathrm{C}-16$ ), oleic (C-20) and tuberculostearic acids (methylated C-20). ${ }^{62}$

The plasma membrane also contains a number of associated components that perform different cell membrane functions, menaquinone for electron transport, carotenoids for photolysis protection, and glcosyl phosphopolyprenols which serve as carriers and intermediates in the process of cell wall biosynthesis. ${ }^{63}$ Porin-like proteins are also essential in the membrane and usually span the entire cell wall to allow the selective 


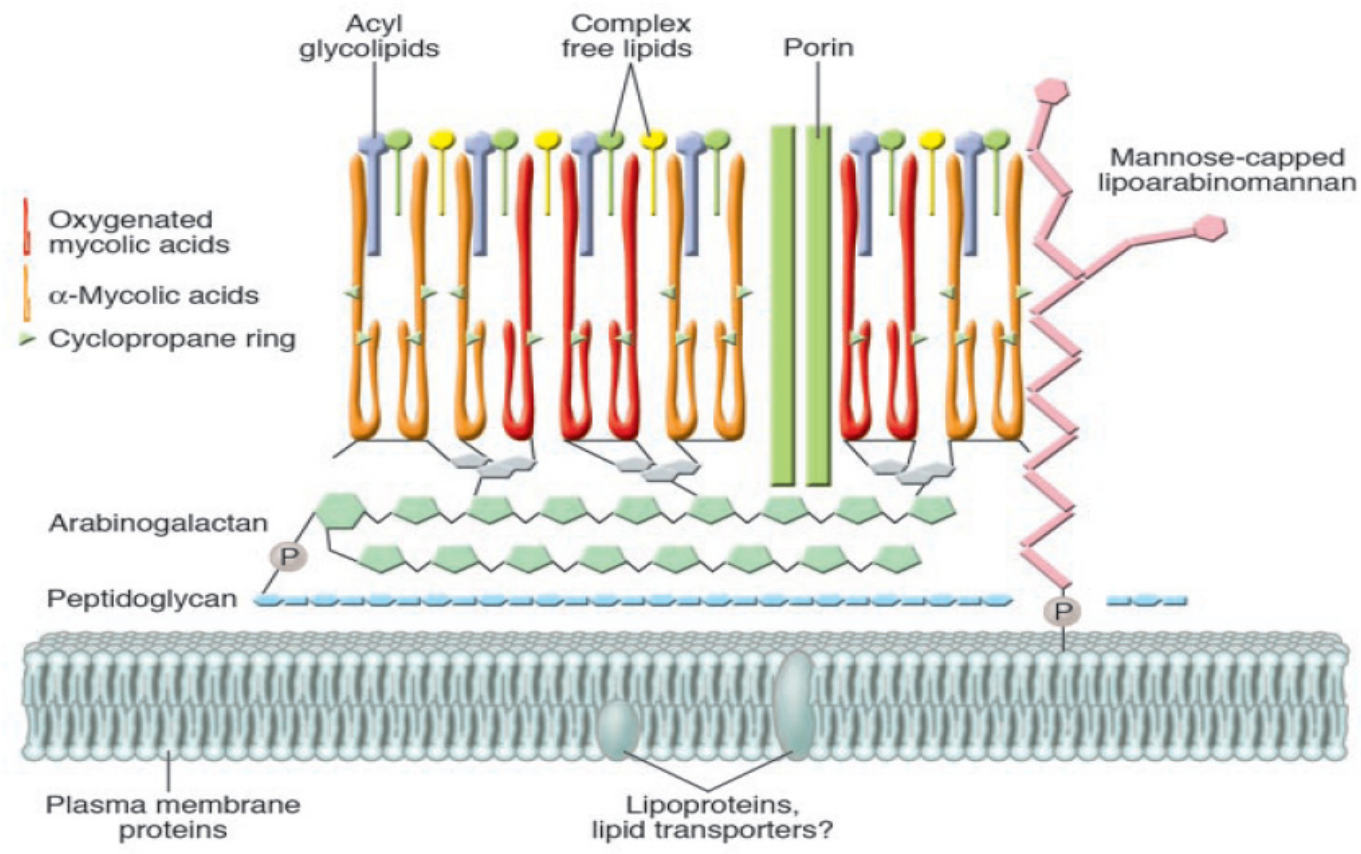

Figure 1.1 Schematic Presentation of the Cell Wall of Mycobacteria

Reprinted with permission. Riley, L.W. Of mice, men, and elephants: Mycobacterium tuberculosis cell envelope lipids and pathogenesis. J. Clinical Invest 11, 1575-8 (2006). ${ }^{64}$

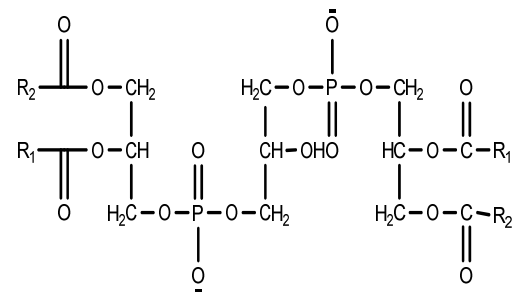<smiles>[R]C(=O)OCC(COP(=O)(O)OC)OC([2H])=O</smiles><smiles>[2H]C(=O)OCC(COP(=O)(O)OC(C)C)OC(=O)O</smiles>

Figure 1.2 Phospholipids of the Mycobacterial Plasma Membrane 
passage of hydrophilic nutrients through the permeability barrier of mycolic acids and the hydrophobic lipids enclosed within. ${ }^{59,60}$

\subsubsection{Phosphatidyl myo inositol mannosides (PIMs)}

These are highly abundant phospholipids that are located in the plasma membrane (mainly the outer leaflet) of actinomycetes. Their chemical structure has been well established and was shown to consist of phosphatidyl inositol which is glycosidically linked at positions 2 and 6 to two mannose residues to form phosphatidyl inositol dimannoside $\left(\mathrm{PIM}_{2}\right)^{65}$ (Figure 1.3). The Mannose residue at 6- position can be further glycosylated with 1-4 mannose residues to form tri, tetra, penta and hexa derivatives $\left(\mathrm{PIM}_{3}, \mathrm{PIM}_{4}, \mathrm{PIM}_{5}\right.$ and $\mathrm{PIM}_{6}$ respectively $) .{ }^{66,67}$ Variability in the acylation patterns of PIMs has been recognized lately and has received considerable research effort. Beside the two lipids chains of the phosphatidic acid, the existence of multi acylated PIMs was reported by several researchers. ${ }^{68,69}$ In fact, among PIMs isolated from M. bovis BCG, tri and tetra acylated $\mathrm{PIM}_{2}$ and $\mathrm{PIM}_{6}$ were found to be the most abundant. ${ }^{66}$

PIMs are known to react with toll like receptors-2 (TLR-2) thus activating the host innate immune response. ${ }^{66}$ Other studies have suggested the involvement of PIMs in the recruitment of natural killer T-cells. ${ }^{70}$ Some of the molecular basis of these activities can be explained through the high affinity interaction between the lipid chain of PIMs and the human $\mathrm{CDb} 1$ receptors. ${ }^{71}$ The hydrophobic nature of this interaction unequivocally explains the loss of biological activity of PIMs if they are subjected to alkaline hydrolysis. $^{72}$

\subsubsection{The Peptidoglycan Mycolyl Arabinogalactan Complex (PG-mAG)}

The covalently bound PG-mAG complex can be isolated from the cells in a pure form and it is described as the cell wall skeleton. A summary of the main structural features of individual parts of the cell wall skeleton is discussed below.

\subsubsection{Peptidoglycan (PG)}

Peptidoglycans provide the bacterial cells with the rigidity necessary to maintain a defined shape. They are universal in their distribution among bacteria with few exceptions such as mycoplasma. ${ }^{73}$ The peptidoglycan of Mycobacteria is classified as AI $\gamma$ type, in which the glycan strands of alternating $\mathrm{N}$-acetyl muramic acid and $\mathrm{N}$-acetyl glucosamine are connected via cross linkage between two peptide chains of L-alanyl-Disoglutaminyl-meso-diaminopimelyl-D-alanine. The two peptide chains are connected between one diaminopimelyl of one strand and D- alanine of the other strand. ${ }^{58}$ This type of PG is common among bacteria but that of Mycobacteria has several characteristics to it. $^{74}$ First, about $30 \%$ of the muramic acid residues are N-glycolylated instead of Nacylated. ${ }^{75}$ Second, the peptide chain link can be between two molecules of Daminopimelic acid (DAP) instead of the more common DAP/D-alanine link. Peptide chain cross linkage in general occurs with a higher than normal frequency in 


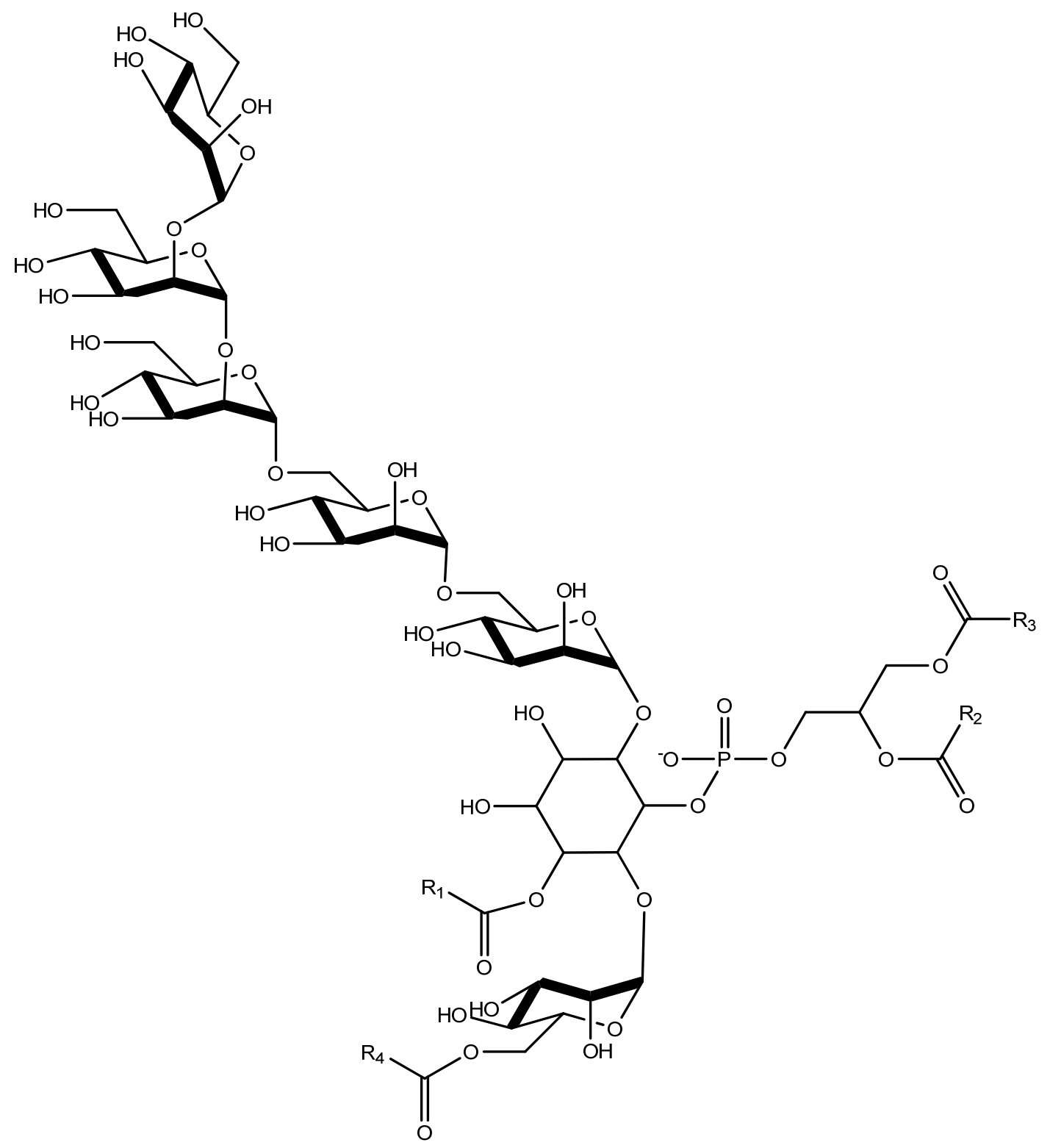

Figure 1.3 Structure of Tetracylated PIM $_{6}$ 
mycobacterial PG (75\% cross- linkage compared to $25 \%$ in case of E. coli) ${ }^{73,76}$ Third, the amino acids of the peptide chain display different chemical modifications, some DAP residues can be amidated while the L-alanine as well as the D-glutamate residues can be substituted with glycine. ${ }^{77}$ The likely purpose of these modifications of the peptidoglycan is to provide the cell wall with more rigidity and chemical stability. ${ }^{73}$

\subsubsection{Arabinogalactan (AG)}

Outside the PG and covalently attached to it, is the complex polysaccharide of arabinogalactan (AG) ${ }^{78}$ The link between $P G$ and AG is thought to be through a disaccharide phosphate linker of rhamnose and $\mathrm{N}$-acylated glucosamine where a furanogalactan residue is linked to the 4-position of the rhammonose residue in the linker region to form a polysaccharide chain of $\beta$-D-Galf-( $1 \rightarrow 5)-\beta$-D-Galf- $(1 \rightarrow 4)-\alpha-1-$ Rhap$(1 \rightarrow 3)-\alpha-d-G l c N A c-1-P$ which then attaches to the PG through about $10 \%-12 \%$ of muramic acid residues of $\mathrm{PG}{ }^{79}$

The building units of the AG complex, i.e. arabinose and galactose, are both in the unusual furanose ring structure. ${ }^{80}$ The galactan chain of AG consists of 25 residues of alternating $1 \rightarrow 5$ and $1 \rightarrow 6$ linked $\beta$-D-Gal $f^{7,81}$ (Figure 1.4). Hepta-arabinan chains are frequently attached to some of the 6 - linked galactose units through a $1 \rightarrow 6$ glycosidic linkage. ${ }^{82,83}$ These arabinan chains are further branched through an $\alpha-3,5$ Ara $f$ residue to two tetra arabinan chains. The short tetra arabinan chains terminate with a branched arabinan moiety of six arabinose units known as Ara6. ${ }^{84}$ In addition to the two basic saccharide units, a succinyl residue or a galactose amine residue can be attached to the $\mathrm{C}$ 2 oxygen of the inner 3,5- $\alpha$-D-Araf residue at the arabinan branching points. ${ }^{84}$ At the terminal end of the Ara6 structure mycolic acids are esterified to position- 6 of the terminal $\beta$-D-Araf.

\subsubsection{Mycolic acids}

The mycolic acids attached to the mAG-PG complex are very long fatty acids that are limited in their distribution to the actinomycetals. They are chemically described as $\alpha-$ alkyl- $\beta$-hydroxy fatty acids with two alkyl branches; a shorter unsubstituted $\alpha$-branch and the longer $\beta$-branch or meromycolate chain. ${ }^{85}$ This structural feature remains valid among Corynebacteria, Mycobacteria and Nocardia but mycobacterial mycolic acids are characterized by having the longest carbon chain with an average length between 70-90 carbons. $^{86,87}$

Variations in the substitution of the meromycolate chain are responsible for the presence of several types of mycolic acids. ${ }^{88-90}$ Unsaturation is the most common substitution among fast growing Mycobacteria as well as non-Mycobacteria actinomycetals. The name $\alpha$-mycolates is used to describe molecules where the chain substitution is either unsaturation or cyclopropation. ${ }^{91}$ In $\alpha$-mycolates the double bonds or cyclopropyl rings can be either in cis or trans configuration with the trans substitution adjacent to a methyl group (Figure 1.5). Other types of mycolic acids usually contain one cycloprpopyl ring together with an oxygenated functional groups like hydroxyl, epoxy, 


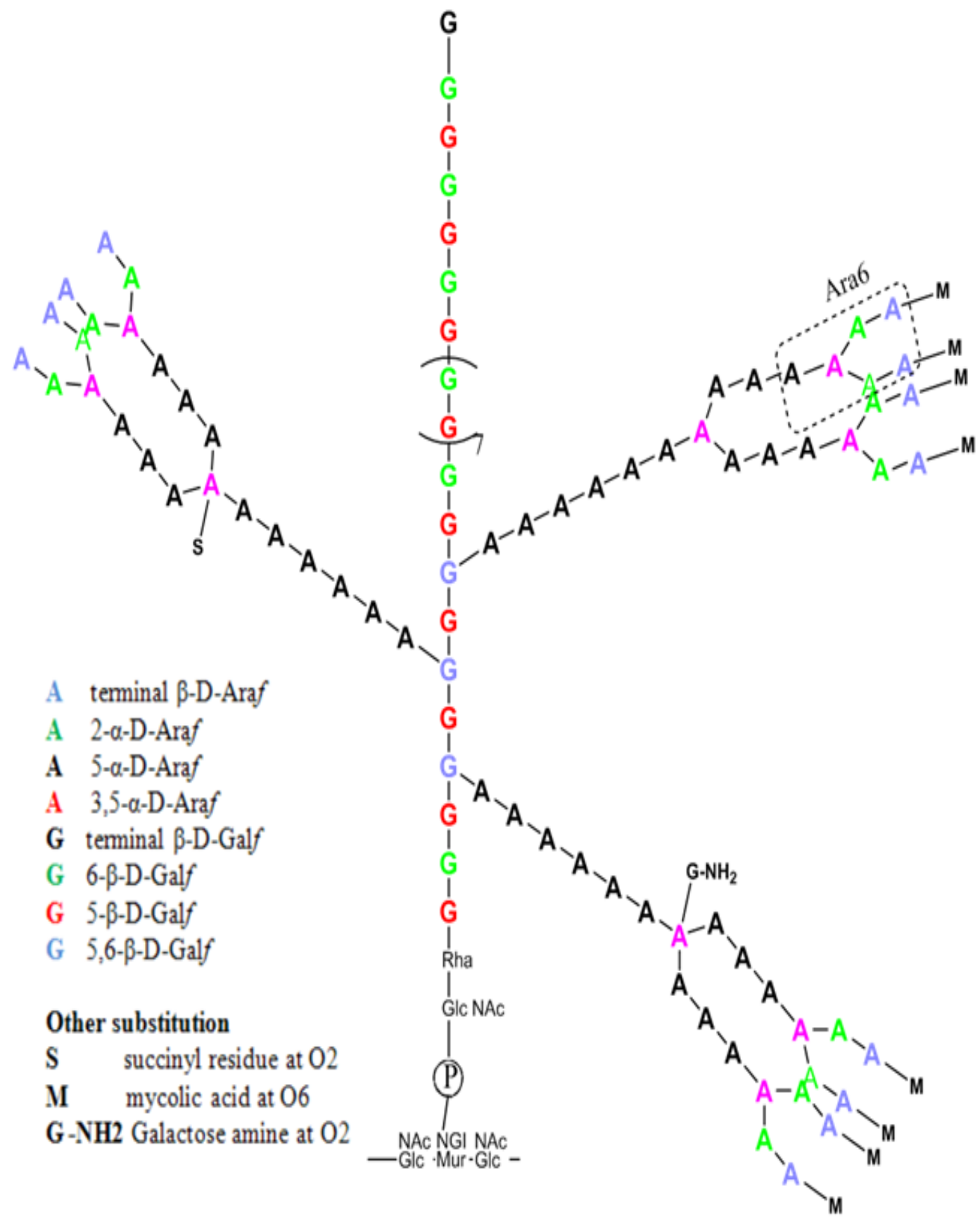

Figure 1.4 Schematic Presentation of the Structure of Mycobacteria AG 
$\alpha$ - mycolate

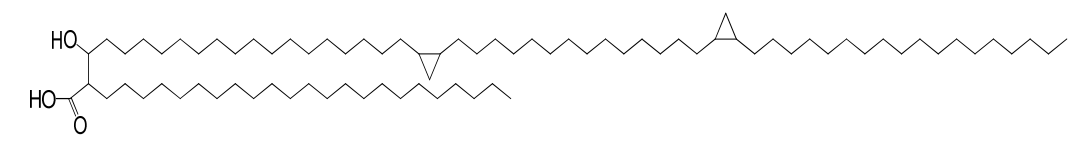

$\alpha_{1}$ - mycolate

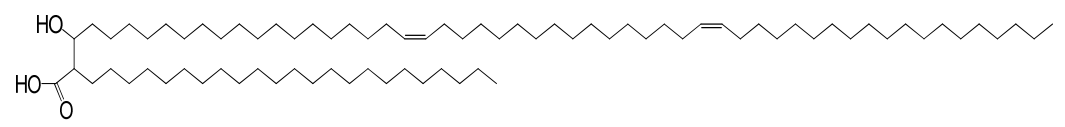

$\alpha_{2}$ - mycolate

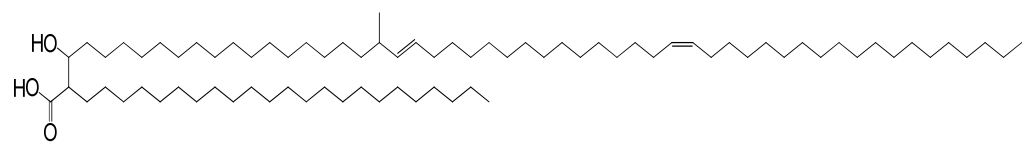

$\alpha$ - mycolate

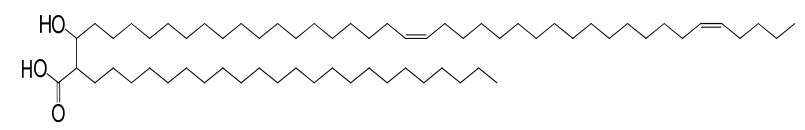

methoxy mycolate

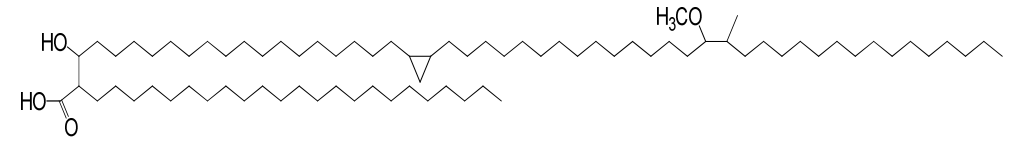

keto mycolate

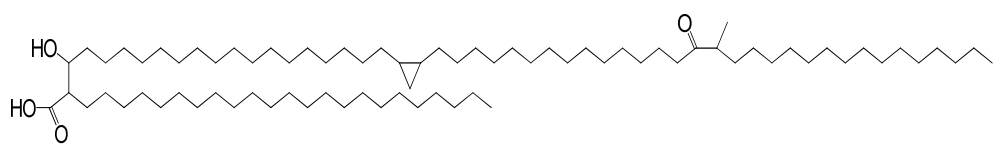

epoxy mycolate

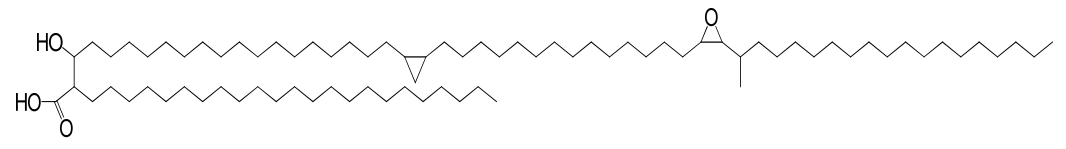

Figure 1.5 Major Types of Cell Wall Mycolic Acids 
keto or methoxy groups and are commonly known as oxygenated mycolic acids. These oxygenated mycolic acids always contain a methyl branch next to the oxygenated function as well. $^{90,91}$

Mycolic acids that terminate the arabinogalactan are tightly packed to form an outer pseudomembrane layer that appears as an electron transparent zone in the electron micrograph. ${ }^{92}$ Variation in both the chain length and the distribution of different types of mycolic acids in a certain species have had implication on the taxonomical classification of actinomycetals. ${ }^{93,94}$

\subsubsection{Extractable Cell Wall Lipids}

Apart from the cell wall skeleton, the cell envelope of Mycobacteria contains some lipids of unusual structures that are believed to play an important role in virulence and pathogenicity. The general agreement is that these lipids are loosely attached to the mycobacterial cells by hydrophobic forces that keep these lipids within the lipid pseudomembrane of mycolic acids. ${ }^{92}$ It was the common belief that these lipids do not exhibit a particular arrangement but recent evidence by improved microscopy techniques has argued for an organized lipid bilayer structure in the outer membrane. ${ }^{95}$

The different chemical nature and structural diversity exhibited by these lipids suggests a different biological role for each chemical class of lipids. In favor of this argument is the fact that certain classes of lipids can be remarkably abundant in one species yet completely absent from others. Even within a single class of lipids, inter-species and inter-strain variations do exist. ${ }^{96,97}$ Over the past few decades, researchers have gathered information that displays the unique structural diversity of the cell wall lipids. The relatively polar glycolipids of the cell wall include trehalose esters, lipooligosaccharides, phenolic glycolipids and glycopeptidolipids. More hydrophobic lipids are also found associated with the cell wall and these include triacyl glycerols, wax esters, mycocerosic and phthiocerol derivatives and acylated glycerophospholipids.

With the wealth of research on the role of different cell wall lipids and the conflicting results it produces, it is crucial to develop a consensus on their biological activities. For example, one of the most ubiquitous glycolipids, known as trehalose dimycolate (TDM), has been associated with serious pathological and immunological effects that contradict its universal distribution among both pathogenic and non pathogenic Mycobacteria. ${ }^{98} \mathrm{On}$ the other hand, it can be argued that subtle differences in the mycolic acids substitution within the TDM molecules among different species can account for some variation in their biological activity. ${ }^{64,99}$ The cell wall fractions also include some proteins and lipoproteins that can be involved in membrane transport functions. A brief description of the major extractable cell wall lipids is outlaid in the following sections. 


\subsubsection{Lipomannans LM and lipoarabinomannans (LAM)}

These are immunogenic lipoglycans that are interspersed within the cell wall and are $t$ thought to be partially surface-exposed. ${ }^{100}$ Degradative analysis of cell wall LAM indicated the presence of glycerol, inositol, arabinose, mannose and phosphate in addition to a variety of fatty acids. ${ }^{80}$ Detailed structural analysis of these glycolipids concluded that they are multiglycosylated derivatives of PIMs which strongly suggests that they may have the same cellular location as PIMs, i.e. they are anchored to the outer leaflet of the cell membrane via their phosphatidyl inositol portion.

Unlike PIMs the mannan part of both LAM and LM is further extended to a backbone of repetitive units of $\alpha-(1 \rightarrow 6)$ D-Man $p$ (Figure 1.6) which are frequently substituted at the $\mathrm{C} 2$ - position (C3-position in few cases) with another Man $p$ residue. ${ }^{101,102}$ In the case of LAM, this mannose-based backbone is further substituted with a number of arabinan side chains of $\alpha-(1 \rightarrow 5)$ D-Ara $f$ units. ${ }^{103}$ These side chains are further substituted at different points with shorter arabinan chains. The arabibnan chains are terminated with one of two arabinan moieties the first is a linear chain of $\alpha-(1 \rightarrow 5)$ Araf $($ Ara4 $)$ and the second is a branched moiety similar to the Ara6 of AG. ${ }^{104}$

Based on the substitution of the terminal arabinose residue, LAM can be classified into three classes. The first is manLAM where the arabinose reducing terminal is capped with one to three mannose residues as it is the case in both $M$. tuberculosis and $M$.

bovis. ${ }^{104-106}$ The second type is most common among fast growing Mycobacteria such as M. smegmatis where the arabinose termini are partially capped with phosphoinositol forming PiLAM. ${ }^{107}$ The third type is devoid of both mannose and phosphoinositol caps such as in M. chelonae and is called araLAM. ${ }^{108}$ On the other hand, Lipomannan (LM) as the name indicates is devoid of any arabinose residues and can be considered as a biosynthetic intermediate of LAM. ${ }^{100}$ Despite that, the two lipoglycans display different immune-modulatory properties. While manLAM is thought to down regulate the inflammatory response to subvert the host immune response and inhibit lysosome phagosome fusion, ${ }^{100,109-111}$ evidence points to a role of LM in triggering a potent proinflammatory response (induction of TNF- $\alpha$, IL-8 andIL-12) and stimulating innate immune response through TLR-2. ${ }^{111,112}$

\subsubsection{Acylated trehalose (ATs)}

Trehalose is a non reducing disaccharide formed through $\alpha, \alpha-1,1$ glycosidic linkage of two $\alpha$-glucose units. The most studied acylated trehaloses are 6,6'-trehalose dimycolates (TDM), also known as cord factor (Figure 1.7). TDM was first reported in $M$.

tuberculosis when many researchers have attributed the virulence of the tubercle bacilli to this molecule as they associated it with the ability of virulent Mycobacteria to form serpentine cords (hence the name cord factor). Further research has proved that TDMs have a more ubiquitous distribution as they exist in other non pathogenic Mycobacteria as well as Corynebacteria and Nocardia. ${ }^{60,97}$ Yet, there is no shortage of reports about the biological activities attributed to TDM that range from inducing Th1 response and initiating granuloma formation to desiccation resistance. ${ }^{98,113,114}$ Results from in vivo 


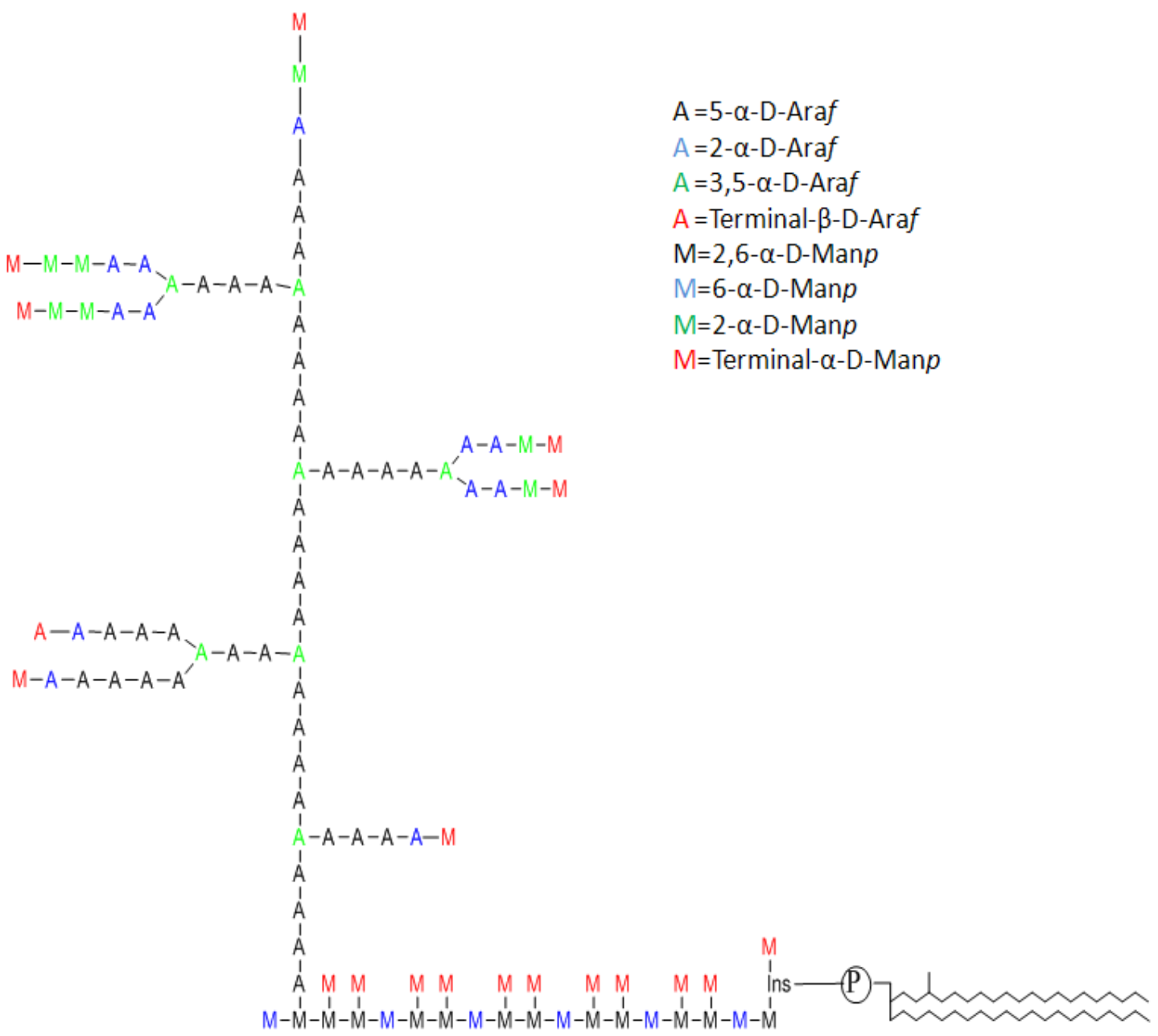

Figure 1.6 Schematic Representation of the Cell Wall manLAM 

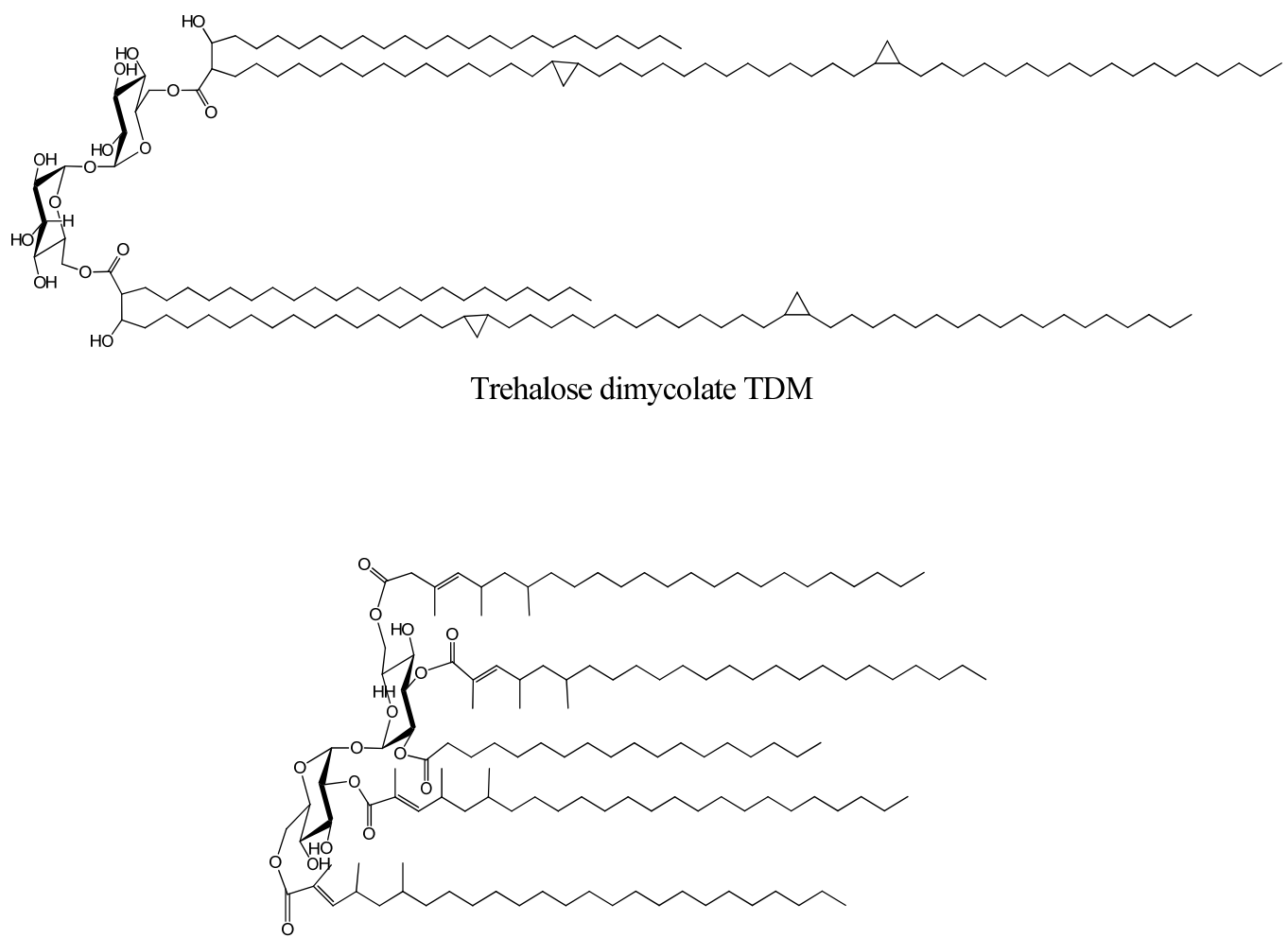

Pentaacylated trehalose PAT

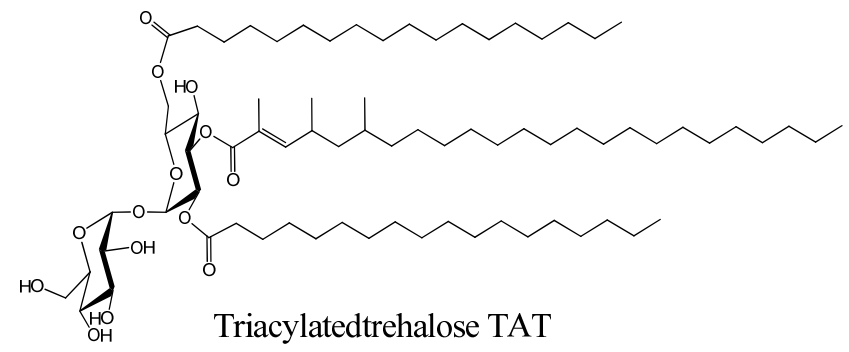

Figure 1.7 Representative Examples of Acylated Trehalose 
experiments to investigate the exact role of TDM in Mycobacteria pathogensis point in many different directions which leaves a lot of doubt about the pathological function of these molecules. It is clearly established, however, that the monomer unit of TDM, glucose monomycolate (GMM), is one of the ligand to human CD1b receptors. ${ }^{71,115}$

Beside cord factor, other forms of ATs exist in some mycobacterial species (some are shown in Figure 1.7). These include trehalose monomycolate (TMM), 2,3-diacyl trehalose, triacyl trehalose and pentaacylated trehaloses. ${ }^{116-119}$ Most of these acylated trehaolse derivatives are esterified with methyl-branched fatty acids such as tuberculostearic, phthieonic, mycocerosic and phthioceranic acids.

Other trehalose esters can be restricted in distribution to certain species. For example Mycobacterium phlei is characterized by the presence of special trehalose phleates, a family of lipids that consists of a trehalose sugar esterified by multiple molecules of polyunsaturated fatty acid (phleic acid). ${ }^{96,120}$ The most abundant of which is the fully acylated trehalose octaphleate which contains 40 double bonds. So far, trehalose phleates have been shown to be restricted in their distribution to M. phlei. ${ }^{63}$

\subsubsection{Sulfated glycolipids (SL)}

Sulfolipids or sulfatides are ATs that were discovered during the search for the acidic substance responsible for the reactivity of virulent $M$. tuberculosis towards staining with the cationic phenazine dye neutral red. ${ }^{121-123}$ They consist of a family of acylated trehalose sulfate the most abundant of which is SL-1 (2,3,6.6'-tetraacyl trehalose-2'-sulfate) ${ }^{121}$ as shown in Figure 1.8. The esterification of trehalose sulfolipids includes always a palmitic or stearic acid at $\mathrm{C} 2$ position while other substitutions are long methyl-branched fatty acids of phthioceranate or hydroxyphthioceranate. Four other sulfolipids were isolated namely SL-I', SL-II, SL-II' and SL-III. ${ }^{63}$

Until now, sulfolipids are thought to be limited in their distribution among Mycobacteria to a limited number of pathogenic species. Interestingly, the avirulent

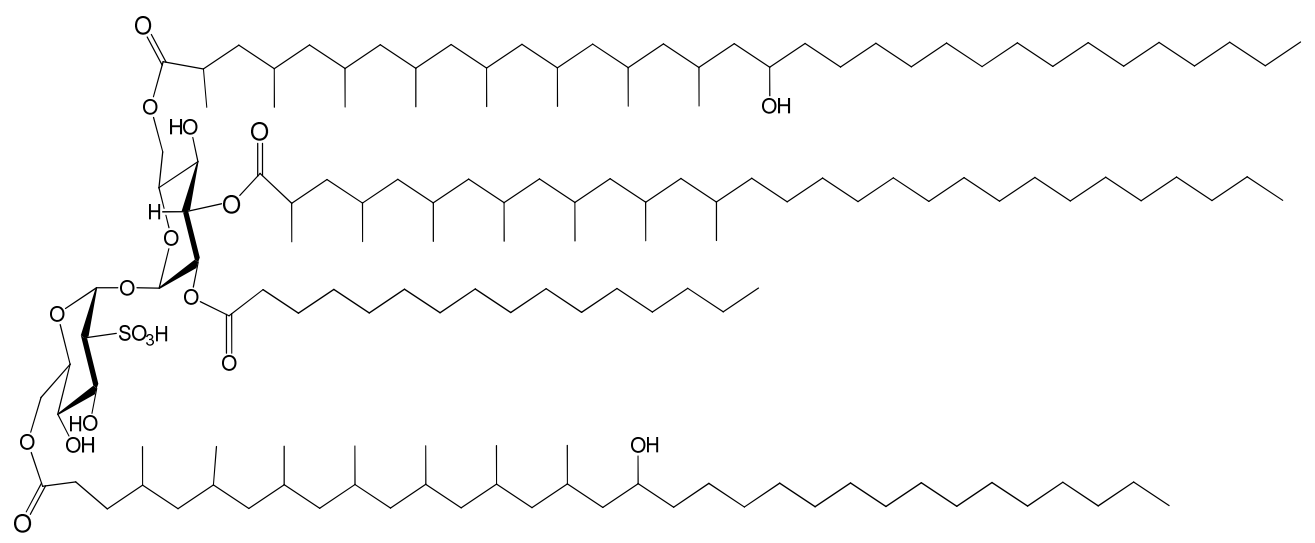

Figure 1.8 Structure of SL-1 
strain M. tuberculosis $\mathrm{H}_{37} \mathrm{Ra}$ was found to be devoid of all major sulfolipids. ${ }^{124}$ Although initial reports indicated a major role of sulfolpids in the virulence of M. tuberculosis, ${ }^{125}$ this claim has been recently refuted by mutation studies that used sulfolipid deficient strains. $^{126,127}$

\subsubsection{Lipooligosaccharides (LOS)}

The term is used to describe a variety of glycolipids that have been identified among several species of Mycobacteria and share a common core structure. The core of these LOS is based on a polyacylated trehalose to which other sugar residues are glycosidically linked. ${ }^{128,129}$ Like most glycolipids, LOS are considered as major antigenic components of the mycobacterial cell wall and their antigenic properties are mainly associated with the terminal sugar. Some of these sugars are very rare and are specific to distinct species like the $\mathrm{N}$-acyl kansosamine sugar isolated from of $M$. kansasii LOS. ${ }^{130}$ The variability among LOS in the types of sugars substituents and their acylation patterns make these compounds valuable tools in serotypying.

LOS are often substituted with methyl branched fatty acids but substitution with other groups have also been reported such as substitution with pyruvic acid in the case of $M$. smegmatis. ${ }^{129,131}$ LOS seem to have a ubiquitous distribution as they have been reported in several pathogenic as well as non pathogenic Mycobacteria including M. kansasii, $M$. malmoense, M. szulgai, M. gordonae, M. butyricum, M. marinum and M. tuberculosis Canetti strain. ${ }^{120,132-136}$

\subsubsection{Phthienoic and mycocerosic acids}

Phthienoic, also known as mycolipenic acids, are odd numbered dextrarotatory methyl branched acids of average carbon chain length between 25 and 31 carbons. ${ }^{63}$ Cason and Sumrell were able to isolate a dozen of these acids and concluded that they have an $\alpha, \beta$ unsaturated acid moiety together with three or four methyl branches in the carbon chain. ${ }^{137,138}$ These fatty acids were originally isolated from the phosphatide fractions of M. tuberculosis but were also found in small number of slow growing Mycobacteria. ${ }^{139,140}$

Some variabilities in the structure of these acids were reported (Figure 1.9). These variations include the presence of hydroxyl group (hydroxyphthienoic acid) and presence of unsaturation (mycolipdienoic acid). It is important to point out that cellular phthienoic acids and their analogs are rarely found in a free state instead they are usually esterified to sugars mainly trehalose or long chain fatty alcohols. ${ }^{116,141}$

Another class of methyl branched fatty acids, mycocerosic or mycoceranic acids, are distinguished from phthienoic acids by being levorotatory. They exist in the cell wall esterified by long chain fatty alcohols, phthiocerols. They are usually 30-32 carbons in length with tetra methyl substitution at positions 2, 4,6 and 8. Being structurally similar to phthienoic acids, both lipids have been implicated in some of the inflammatory characteristic of tuberculosis lesions. ${ }^{142}$ 


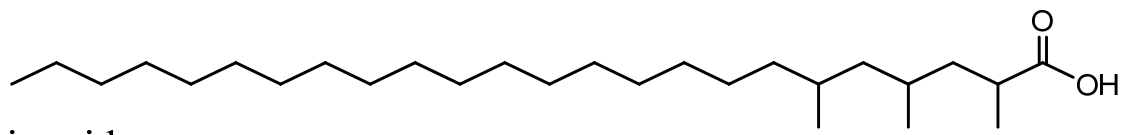

Mycolipanoic acid

Mycolipenoic acid

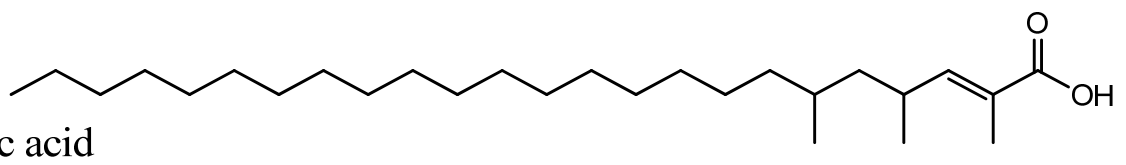

Phthieonic acid

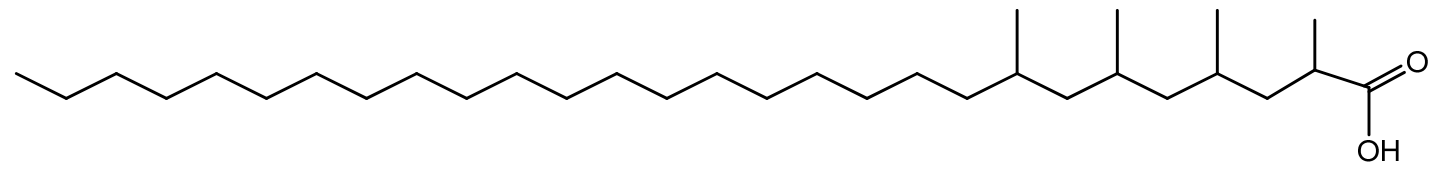

Mycocerosic acid

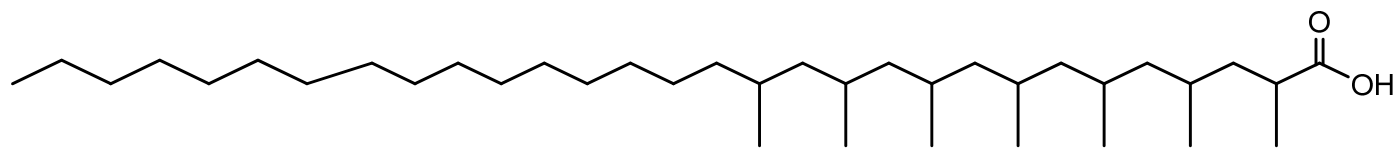

Phthioceranic acid<smiles>CCCCCCCCCCCCCCC(O)CC(C)CC(C)CC(C)CC(C)CC(C)CC(C)CC(C)C(=O)O</smiles>

Hydroxyphthioceranate

Figure 1.9 Examples of Phthienoic and Mycocerosic Acids 


\subsubsection{Phthiocerol diesters}

The phthiocerol family consists of a mixture of long chain $\beta$ diols that exist in the mycobacterial envelope in diester form. Closely related to this family are phenol phthiocerols which are basically aromatic derivatives of phthiocerol ${ }^{143,144}$ (Figure 1.10). Phthiocerol and their aromatic derivatives are found only in the cell wall of few Mycobacteria usually esterified to the multimethylated fatty acids mentioned above (namely mycocerosic and phthienoic acids). This group of lipids is produced mainly by slow growing pathogenic Mycobacteria which include M. tuberculosis, M. bovis, M. kansasii, M. marinum and M. ulcerans. ${ }^{145}$ Also, they were reported in the uncultivable $M$. leprae and the non pathogenic M. gastri. ${ }^{145}$

In order to validate the role of phthiocerol derivatives in Mycobacteria virulence, Goren and colleagues have compared the virulence and pathogenicity of M. tuberculosis $\mathrm{H}_{37} \mathrm{Rv}$ and a mutant deficient in the synthesis of phthiocerol esters in aerogenic infection model using Guinea pigs. ${ }^{146}$ The mutant strain was less virulent and was drastically unable to maintain the surface tubercles which were almost cleared within 50 days. ${ }^{146}$

\subsubsection{Phenolphthiocerol glycolipids (PGL)}

This group of lipids carries a great structural similarity to the phthiocerol diesters but they are synthesized by only a limited number of Mycobacteria species and some specific strains of certain species. ${ }^{59,147}$ The chemical structure of these lipids consists of the same core as phthiocerol diesters where the phthiocerol chain is terminated by a phenolic ring but in PGLs this ring is further substituted through glycosidical linkage with one or more sugar residues (Figure 1.11).

When first reported, PGL were named mycosides. Mycosides A, B and G were isolated from M. tuberculosis strain Canetti, M. bovis and M. marinum respectively and were shown to have very similar aglycon part. ${ }^{145,148,149}$ However, mycoside A is terminated with a trisaccharide of methylated sugars, ${ }_{150} 151,152$ while mycoside $\mathrm{B}$ and $\mathrm{G}$ are terminated with a single sugar residue, 2-O-methylrhamnose and 3-O-methyl rhamnose respectively. ${ }^{145,148}$ The use of the term mycosides has faded and now these molecules are more likely to be called phenolic glycolipids (PGL). Recently, evidence has suggested the association of PGL with increased virulence of some strains of $M$. tuberculosis due to some immune-modulatory properties linked to this molecule. ${ }^{153}$

\subsubsection{Glycopeptidolipids (GPL) or "mycoside C"}

Glycopeptidolipids were isolated initially from M. avium, M. intracellulare and M. scrofulacceum (MAIS subgroup) and later more lipids with similar structure were isolated from other Mycobacteria from different clades. ${ }^{154,155} \mathrm{C}$-mycosides are generally characterized by a peptide core of D-aminoacids mainly D-phe-D-alloThe-D-Ala and this small peptide sequence is usually terminated at the carboxy end with an L-alaninol or ethanolamine substituent ${ }^{156}$ (Figure 1.12). The "tetrapeptide core" is further substituted through its $\mathrm{N}$-terminus, the D-phenylalanine residue, to a long chain fatty acid. ${ }^{157}$ 


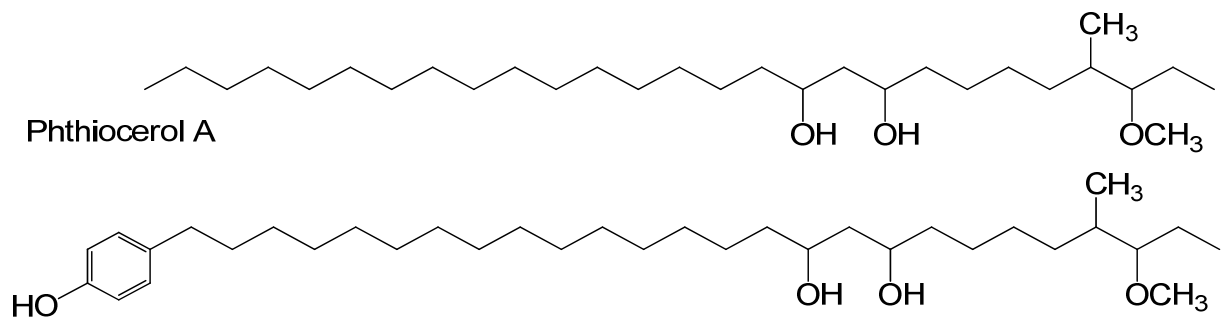

Phenol phthiocerol A

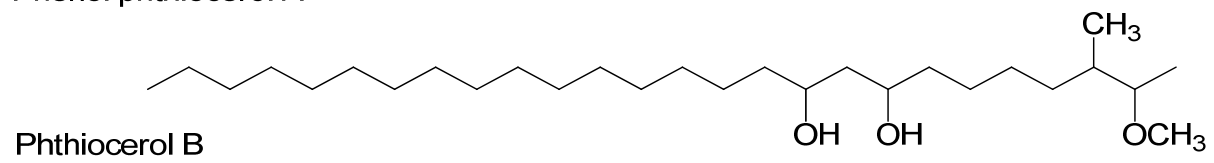<smiles>CC(C)C(C)CCCCC(O)CC(O)CCCCCCCCCCCCCCCc1ccc(O)cc1</smiles>

Phenol phthiocerol B<smiles>CCCCCCCCCCCCCCCCCC(O)CC(O)CCCCC(C)C(=O)CC</smiles>

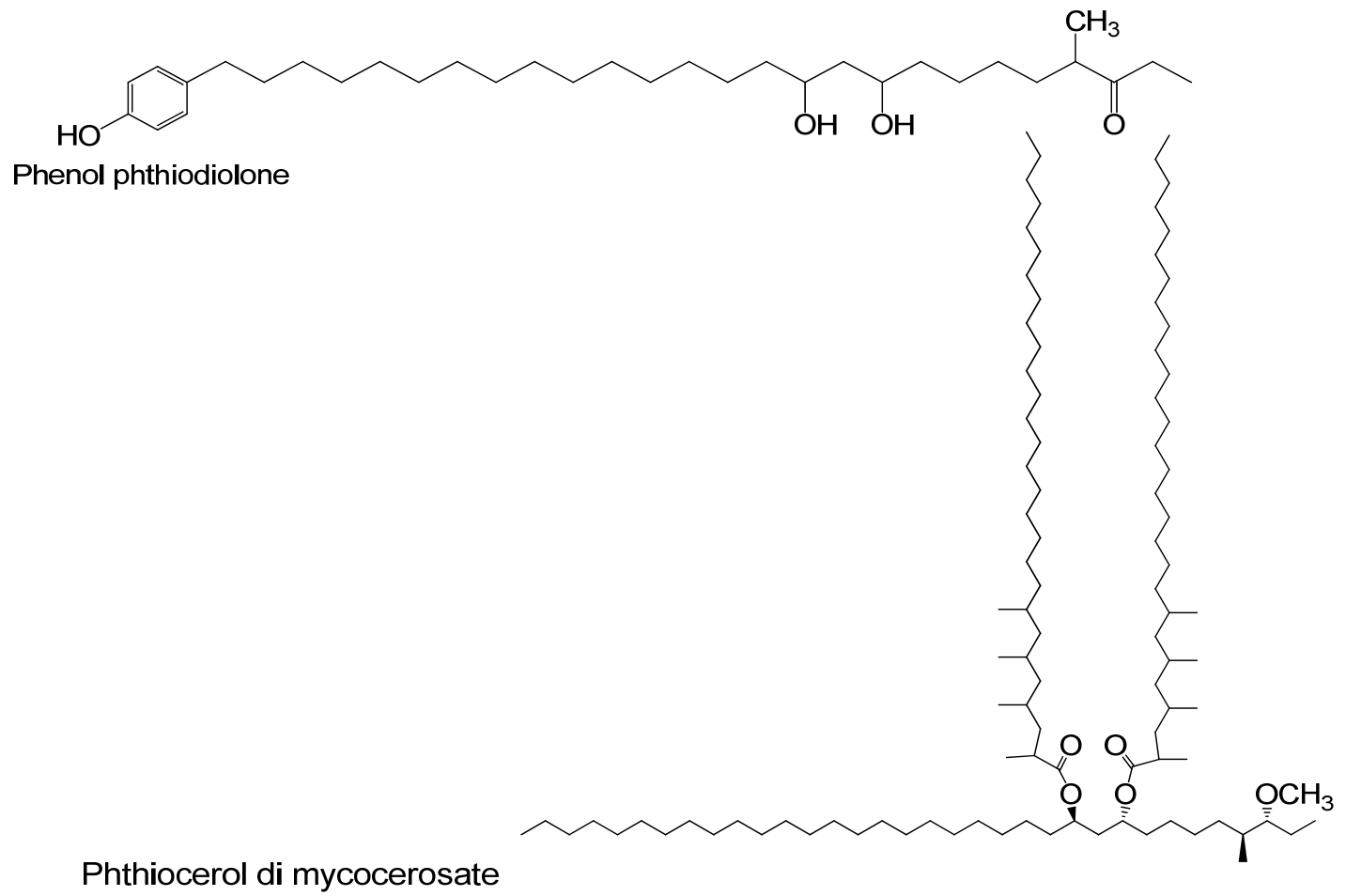

Figure 1.10 Structure of Phthiocerol Derivatives 


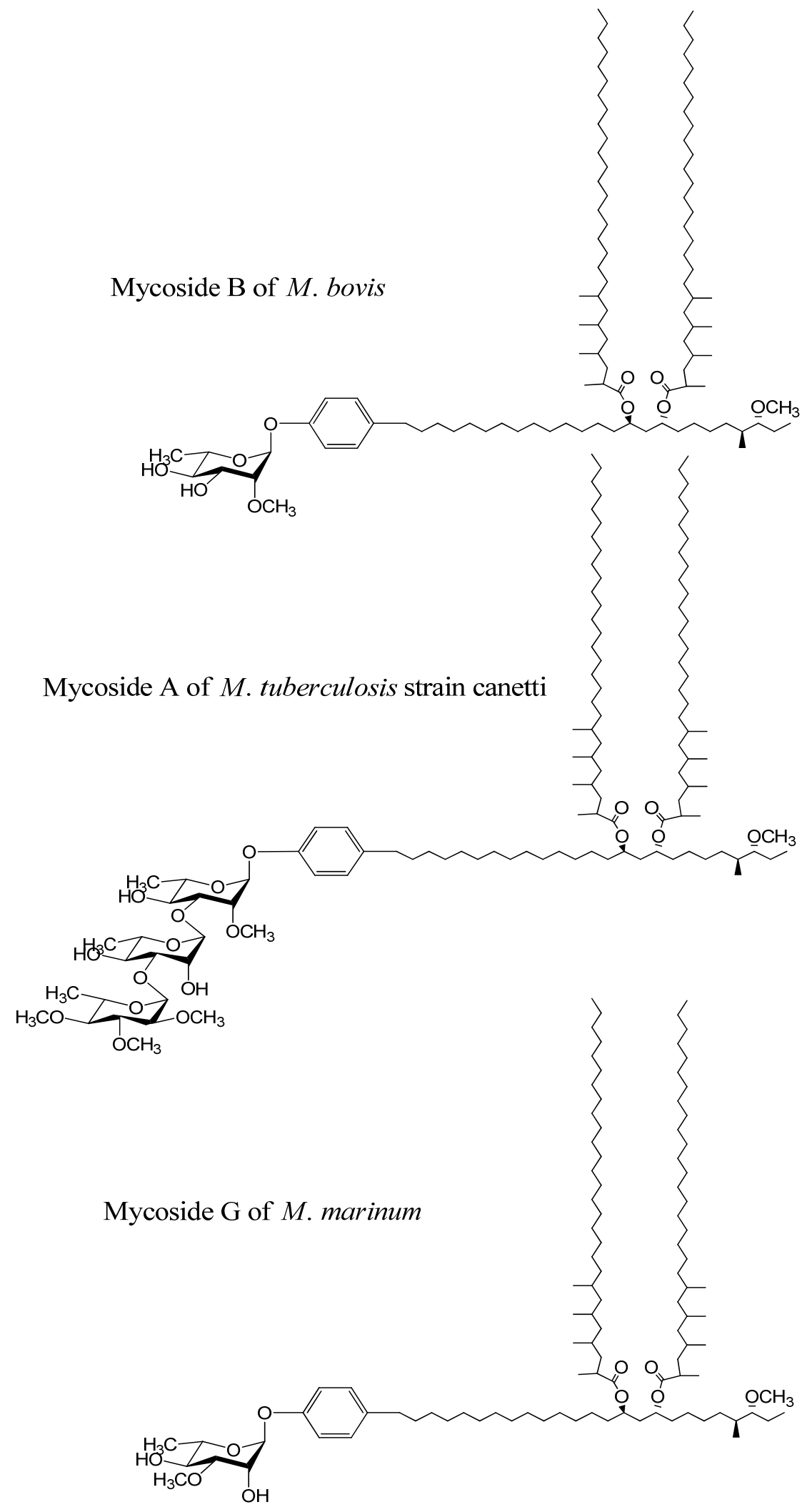

Figure 1.11 Structures of Some PGL from Different Mycobacteria 


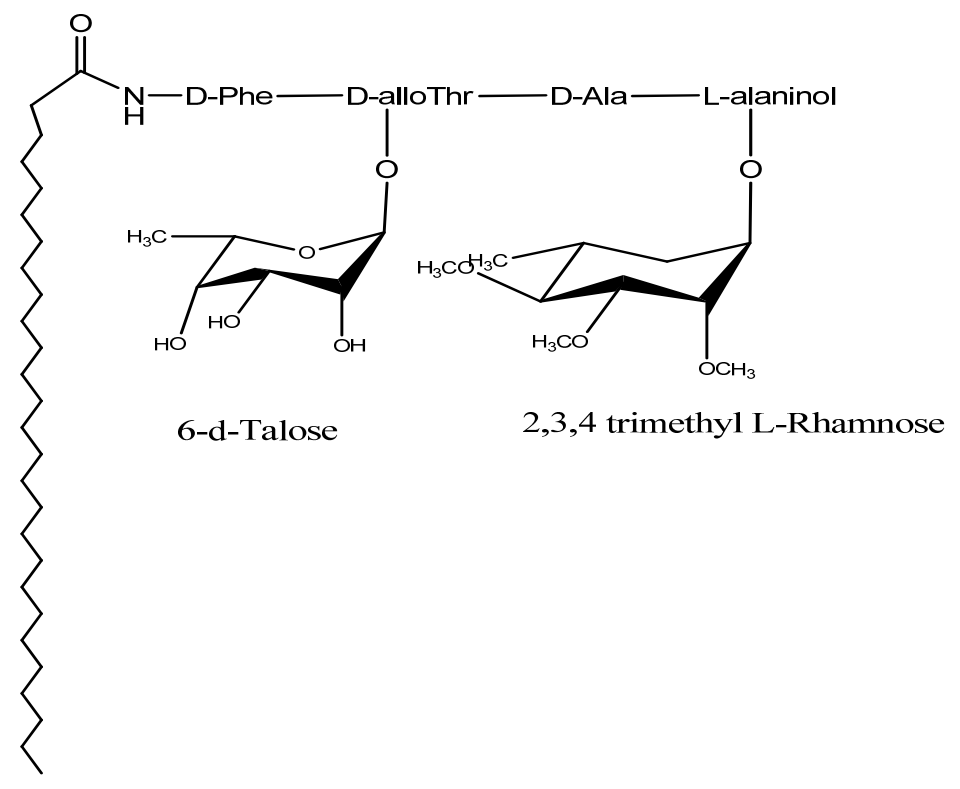

Figure 1.12 The Tetrapeptide Core of GPL

There are two main sites for glycosylation in the PGL core. The first one is at the hydroxyl group of L-alaninol which is usually monoglycosylated with O-methylated -Lrhamnose and the other is at the D-allotheronine site where one or more sugar residue can be attached starting with a 6-deoxy L-talose. ${ }^{155,157}$ The presence of an oligosaccharide attached to the L-talose residue is associated with eliciting a strong strain specific immune response to $M$. avium and $M$. avium complex. ${ }^{96}$ Thus these oligosaccharides are the main tool used for serotyping $M$. avium complex clinical isolates. ${ }^{16}$

\subsubsection{Triacyl glycerols (TAG)}

Triacyl glycerols constitute about 5-10\% of the cellular mass of M. tuberculosis when it is grown in the presence of glycerol. ${ }^{158}$ However, this percentage is greatly reduced when the glycerol in the media is totally replaced by glucose. ${ }^{159,160}$ TAGs are ubiquitous among all Mycobacteria and can be extracted from both the cell wall fraction of the bacilli as well as the cytoplasmic fraction. ${ }^{7}$ The accumulation of TAG in fat bodies or lipid vacuoles has been observed in M. tuberculosis, M. marinum, M. bovis, M. kansasii as well as Corynebacterium sp. when their growth was stimulated by the addition of a lipid substance in the growth media such as Tween $80 .^{7}$

The acyl substitution of mycobacterial TAG exhibits little variation among different species. Position-1 is often substituted with stearic, tuberculostearic or oleic acid. Position-2 is usually esterified with C16 fatty acids while longer chain fatty acids C20C32 are located in position-3. ${ }^{161}$ The metabolic turnover for TAG was found to be very rapid for actively grown bacteria which suggest that they may serve as biosynthetic precursors or temporary energy storage for the bacterial cell. ${ }^{63}$ 


\subsubsection{Mycobactins and carboxymycobactins}

Mycobactins (Figure 1.13) were discovered in the 1950s as the growth factors necessary to cultivate $M$. avium subsp paratuberculosis. ${ }^{162,163}$ They are hybrid peptidepolyketide iron chelating compounds secreted by Mycobacteria to sequester iron during iron limiting conditions such as in the case of competing with its host for a limited iron supply.

The basic iron chelating nucleus of these molecules is 2-hydroxyphenyloxazoline ring which is biosynthesized from salicylic acid condensation with serine or threonine residue. ${ }^{164}$ The serine residue is further linked to a small chain of two lysine residues and one hydroxyl acid. To attain lipid solubility, the side chain nitrogen of one of the lysine residues is substituted with long alkyl chain (C20 or longer). Mycobactins can bind one iron atom per molecule through the coordination of the iron atom by the two hydroxamic acid groups and the hydroxyl group of the salicylic acid residue. ${ }^{164,165}$

Several mycobactins have been isolated from different Mycobacteria and were designated by letters e.g. mycobactins P, T, S, M and H. ${ }^{163,166,167}$ Each species produces its own collection from these mycobactins. Carboxymycobactins are chemical analogues of mycobactin molecules that are excreted outside the cells (exomycobactins). To improve their water solubility, the long alkyl chain is replaced in carboxymycobactins by a shorter chain that terminates with a carboxylic group. ${ }^{168,169}$

\subsubsection{Carotenoids}

These colorful polyene compounds (Figure 1.14) are usually associated with plants and fungi that are capable of photosynthesis. ${ }^{170}$ However, some species of Mycobacteria demonstrate the ability to produce these caratenoid pigments either in a constitutive (scotochromogens such as M. phlei and M. gordonae) or inducible way (photochromogens such as M. kansasii and M. marinum. ${ }^{7,63}$ The biosynthesis of the $\beta$ carotene pigments from isoprenene units includes several intermediates such as the colorless phytoene and the pink colored lycopene.

The distribution of these carotenoids among Mycobacteria depends on both the species and its growth conditions. For example, M. kansasii which produces extracellular $\beta$-carotene crystals is thought to produce mainly oxygenated carotenoids (Xanthins) only when they are exposed to lights. ${ }^{7}$ These pigments are believed to protect cells against oxidation that can be induced by UV exposure or chemical dyes such as toluidine blue. ${ }^{171}$

\subsubsection{Menaquinones}

Menaquinones, 2-methyl-3polyprenyl-1,4 naphthaquinone, are key components of the respiratory chain of all Gram positive bacteria including Mycobacteria. Menaquinones nomenclature (Figure 1.15) follows the general formula of menaquinine- $\mathrm{X}\left(\mathrm{H}_{\mathrm{y}}\right)$, where $(\mathrm{X})$ is the number of isoprene units attached at position 3 of the ring and (y) is the number of hydrogen atoms that saturate that chain. ${ }^{172}$ Variation in the number of isoprene units 


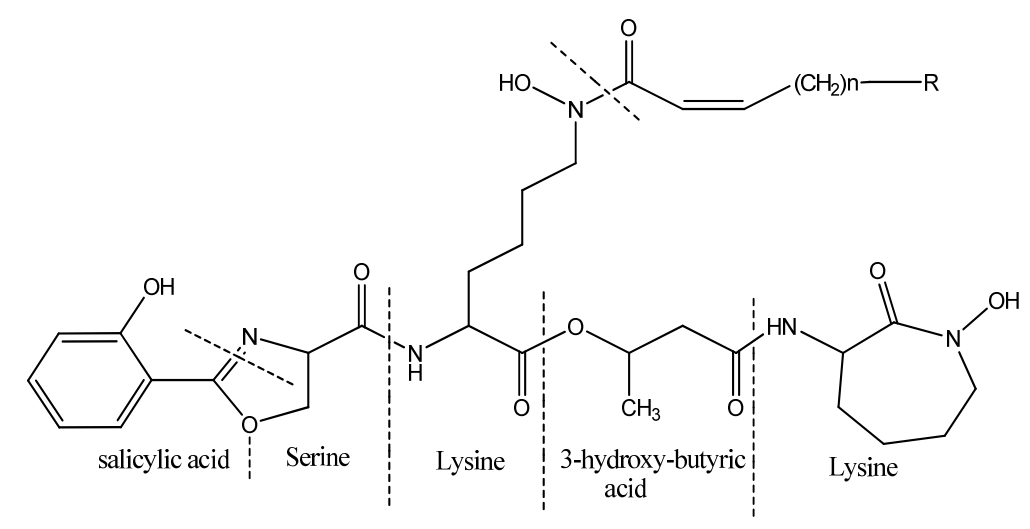

Figure 1.13 General Structure of Mycobactins

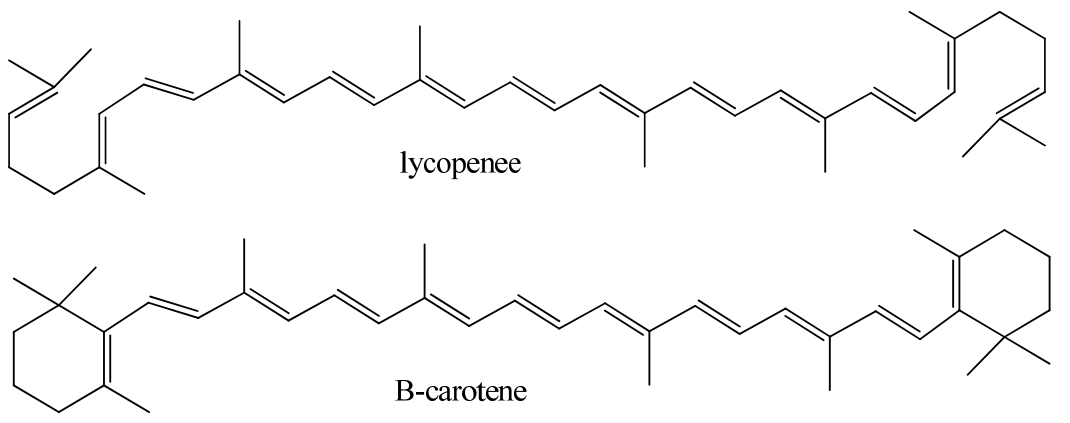

Figure 1.14 Structure of Some Examples of Carotenoids

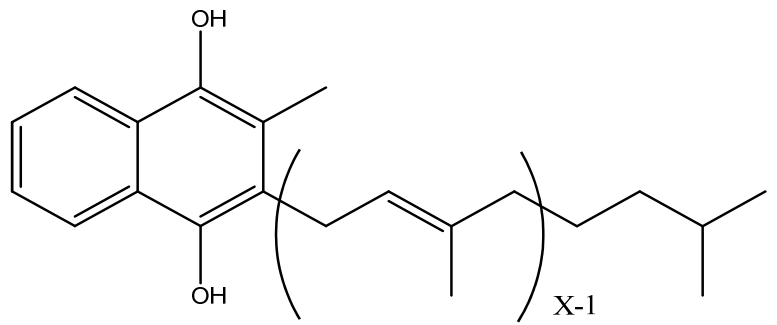

Figure 1.15 General Structure of Menaquinone 
and the degree of unsaturation led researchers to use menaquinones as taxonomical markers in the classification and identification of actionmycetes. ${ }^{172}$

Among Mycobacteria, menaquinone-9 $\left(\mathrm{H}_{2}\right)$ is the most abundant and has the widest distribution with limited amounts of menaquinone- $8\left(\mathrm{H}_{2}\right)$ and menaquinone-7 $\left(\mathrm{H}_{2}\right)$ reported in some species. This also distinguish Mycobacteria from Corynbacterium dephteriae which contains mainly menaquinone- $8\left(\mathrm{H}_{2}\right){ }^{173}$

\subsection{Role of the Mycobacterial Cell Wall in Virulance}

The unusual complexity of the Mycobacteria cell wall necessitates that a significant part of its genome is dedicated to genes that participate in the biosynthesis of all of its unique components. This in turn imposes a metabolic burden on the bacterial cell and requires the devotion of large amount of energy for its assembly. Despite such evolutionary pressure, the structure of the cell wall is well conserved among most Mycobacteria. All of this suggests the importance of each single component of the cell wall to the survival of these organisms.

As previously discussed, there are several unique characteristics to the mycobacterial cell wall. For example, in the mycobacterial PG, both the glycan and the peptide branches have been modified to confer superior rigidity and chemical stability to the cell wall. ${ }^{73}$ The AG complex adds more strength to the cell wall and serves another important function by anchoring the mycolic acids to the cell skeleton. ${ }^{60}$ These Mycolic acids are the main permeability barrier for the penetration of most chemotherapeutic agents thus rendering Mycobacteria resistant to most commonly used antibiotics and antiseptics. ${ }^{59,60}$

Loosely bound mycobacterial lipids that are located at the surface of the bacterial envelope can easily diffuse and interact freely with the host's immune system. Some of the glycolipids associated with the cell wall have already been shown through in vitro and less frequently in vivo experiments to interfere with the host defense mechanisms and many are thought to represent potential virulence factors. For example, TDM was shown to produce toxicity in mice but not in other animal species. GPL are well recognized as antigens that are used for serotyping of $M$. avium complex and are thought to enhance the survival of $M$. avium inside the macrophages. ${ }^{120,155}$ Phenolic glycolipids (PGL) are known as the main antigenic component of $M$. leprae and are now thought to play an important role in the hypervirulence of $M$. tuberculosis W-Beijing strain. ${ }^{143,144,153}$ They have also been proved to posses the capability of modulating the early host immune response. ${ }^{174}$ In most of these cases however, clear undisputable evidence is lacking, which underscores the importance of more thorough studying of these exotic molecules preferably through the use of a more global approach.

Overall, there is no shortage of evidence to indicate a significant role of the cell wall in the virulence and pathogenesis of Mycobacteria. Many in vivo experiments have demonstrated a powerful host immune response upon the injection of different cell wall components. ${ }^{50,98,100,111,113,120,155,175-177}$ In fact, cell free preparations of M. bovis BCG cell 
wall skeleton have demonstrated some anti neoplastic properties and have recently found a place in immunotherapeutics. ${ }^{178-180}$ The immunostimulatory activity of M. bovis BCG cell wall preparations has been previously attributed to $\mathrm{TDM}^{181,182}$ and in a more recent study to a newly discovered monomycolated glycerol lipid. ${ }^{183}$

\subsection{Mycobacterial Cell Wall as Diagnostic and Taxonomic Tool}

Long before the rise of rapid genomic sequencing and other genetics tools, the characterization of Mycobacteria was based mainly on the biochemical analysis of its cell wall components. ${ }^{184}$ The most abundant lipids of the cell wall, mycolic acids, exhibit enough structural diversity among different Mycobacteria which led to the use of mycolic acid patterns obtained through different chromatographical techniques as biochemical criteria for the taxonomical classification of Mycobacteria. ${ }^{86,93,185}$

Few decades ago, simple chemical hydrolysis of the mAG complex followed by separation with thin layer chromatography (TLC) was used to resolve the pool of mycolic acid in two directions. The 2D-TLC patterns produced through these procedures can be diagnostic for every species. ${ }^{185}$ This technique has found some application in the mycobacteriology research for a certain period of time. ${ }^{186}$ Later with the spread of non tubercular mycobacrterial infections, the need for a powerful diagnostic tool pushed for further improvement of such concept through the use of GC and HPLC. Both methods afforded a much better resolution of the mycolic acid pool and were thus used as a reliable tool in both the taxonomy of Mycobacteria and the characterization of clinical isolates from tuberculosis patients. ${ }^{94,184,187}$

\subsection{Analysis of the Mycobacterial Cell Wall}

\subsubsection{Analysis of Peptidoglycan Structure}

The general structure of PG shows relatively little variability among bacterial species. Routine procedures have been used to detect the amino acid sequence of the peptide chain and the saccharide units of the glycan chain. Due to its location beneath complex layers of lipids and polysaccharide, several procedures including delipidization, deproteinzation, and lysozyme digestion are necessary before PG analysis. ${ }^{188}$

Both enzymatic and chemical hydrolysis are used to break down the cell wall skeleton to yield smaller analyzable fragments of the PG polymer. Subsequent chromatographic procedures are then used to separate the oligosaccharide and the peptide fragments and these fragments are then sequenced through HPLC. ${ }^{58}$ Earlier attempts have used Dcycloserine to inhibit peptidoglycan crosslinking allowing the accumulation of peptidoglycan precursors to facilitate their analysis. However, such treatment resulted in some artifacts that led to few misinterpretation of the PG structure of Mycobacteria. ${ }^{73}$ Later, milder hydrolytic procedures and coupling of the HPLC to advanced MS 
techniques have provided a more quantitative analysis of the peptide and saccharide residues of the PG macromolecules which is responsible for our current understanding of the special feature of mycobacterial peptidoglycan. ${ }^{74,75}$

\subsubsection{Analysis of Arabinogalactan Complex}

\subsubsection{Degradative analysis}

The original strategy for AG analysis included harsh chemical hydrolysis to release the arabinogalactan complex from the cell wall through delipidation and hydrolysis with $1 \mathrm{~N}$ sodium hydroxide. ${ }^{80}$ The hydrolytic step is followed by neutralization, dialysis against pure water and AG precipitation by $80 \%$ ethanol. Successive column chromatography on Dowex and DEAE cellulose removes proteins and yields pure fraction of AG and arabinomannan. Further steps of hydrolysis and derivatization are then necessary to break down the polymers into small sugar fragments that can be analyzed using GC/MS or HPLC/MS. ${ }^{80}$ These long analytical procedures require a large cell mass of about 800 grams. Yet, a lot of information regarding the sugar linkage and the branching pattern of the cell wall polysaccharides can be lost during the harsh chemical treatment.

A milder protocol was introduced by Daffe et al in 1990 that included the production of high quality cell wall preparation through sonication and treatment with surfactant such as triton-x. ${ }^{81-83}$ Extraction with SDS was then necessary to remove associated proteins. The cell wall preparation produced was per-O-methylated using dimethyl sulfinyl carbanions then partially hydrolyzed with trifloroacetic acid. The partial hydrolysis resulted in random glycosidic cleavage at different positions of the AG chain to produce different fragments. Upon reduction of these fragments with $\mathrm{Na} \mathrm{BD}_{4}, 43$ different alditol fragments were produced which can be differentiated through analysis via GC/MS. ${ }^{81}$ Through this methodology, linkages and branching within the AG complex were elucidated. ${ }^{79}$ Later, the same method with few modifications was used to establish the type of linkage between the PG and the mAG complex.

\subsubsection{Solid state NMR HR-MAS for elucidation of $A G$}

The previously discussed procedures have established the structure of AG, its sugar composition, linkage type and branching pattern. This well documented research paved the way for a faster method for screening the structure of AG without long hydrolytic procedures through the use of solid state NMR spectroscopy. While solution NMR was used to establish the configuration and linkage type of the predigested fragments, high resolution magic angle spinning (HR-MAS) can be used with live bacterial cell to analyze intact AG molecule through the production of ${ }^{1} \mathrm{H}-{ }^{13} \mathrm{C}$ HSQC maps. ${ }^{189,190}$ Due to the dynamics of HR-MAS acquisition, the HSQC spectrum of live bacteria preferentially contains signals of arabinogalactan and lipoarabinomannan rather than lipids and mycolic acids. This is due to several factors concerning the short T2-relaxation time of lipids and their slow molecular tumbling in the absence of organic solvents. 
Different residues of arabinose and galactose at specific branching points and with variable substitution were represented by distinct signals in the HR-MAS spectrum. ${ }^{189}$ These signals can be used as indicators for the presence or absence of specific branching points or substituents. The method requires supplementing the culture with ${ }^{13} \mathrm{C}$-carbon source like glucose and glycerol to obtain good quality spectra in a reasonably short time. After enrichment, 30-40 mgs of culture is sufficient for simultaneous structural analysis of both AG and LAM. ${ }^{189,190}$

\subsubsection{Free Lipid Extraction}

Any biochemical approach for studying lipid composition and function has to start with an extraction process to remove the lipids from the cell wall. Two main methodologies have been used for this purpose. First is the Folch's protocol developed by Jordi Folch in 1956 which uses a mixture of chloroform: methanol 2:1 v/v for lipid extraction. ${ }^{191}$ As he described it, an excessive amount of solvents is usually used for extraction (twenty times the volume of the cell pellet). Then the extraction step is followed by washing steps using a solvent mixture of chloroform: methanol: water $3: 48: 47$. This ratio is critical to reduce loss of the lipids to the aqueous layer that separates from the lipid organic layer upon centrifugation. ${ }^{191}$

The second protocol is the method developed by Bligh and Dyer in 1959 which was proposed as a modification of the Folch's to avoid excessive solvent use. ${ }^{192}$ This protocol is better suited for extraction of tissues with more moisture and low lipid content and was proved to be more efficient in extracting polar lipids. It was also simplified from the Folch's protocol to avoid the multiple washing steps through using an extraction solvent with higher methonal ratio then adding more chloroform and water to achieve biphasic solution. Lipid extraction is first achieved using a mixture of chloroform:methanol 1:2 . Subsequently, chloroform and water are added to the final lipid extract to bring the total solvent ratio to chloroform:methanol:water 2:2:1.8 which renders the extract biphasic. The two layers are then separated by centrifugation. The upper aqueous upper layer (devoid of lipid content) is then discarded.

It is to be noted though that both protocols were originally designed for the extraction of membrane lipids from animal tissues which are not covalently bound to the cells. ${ }^{191,192}$ In the case of Mycobacteria both methods can serve the purpose of extracting only loosely attached free lipids. In order to perform mycolic acid analysis, the extraction procedures has to be preceded by a step of alkali or acid hydrolysis to release the covalently bound mycolic acids from the arabinogalactan complex.

\subsubsection{Mycolic Acid Extraction}

Mycolic acids in Mycobacteria are present in two forms; A minor fraction is esterfied to sugars mainly in the form of trehalose mono and dimycolates, while most of the cell wall mycolic acids are covalently bound to the PG-mAG complex. ${ }^{59}$ In order to analyze 
the total mycolic acids pool, the covalently bound fraction has to be released in a free extractable form. This is achieved through either acid or alkaline hydrolysis. Alkaline hydrolysis (saponification) produces the highest yield and is the most frequently used. $^{90,94,187}$ It includes three consecutive steps of hydrolysis, neutralization and extraction. Methanolic or ethanolic potassium hydroxide can be used for saponification followed by acidification of the saponified wax with $\mathrm{HCl}$ to release mycolic acids. ${ }^{193} \mathrm{At}$ this point, the free mycolic acids are readily extractable with an organic solvent such as diethyl ether or chloroform. ${ }^{193}$ For analytical characterization by GC or HPLC, the mycolic acids have to be derivatized to their methyl esters. ${ }^{194}$ Esterification is performed by a methylating agent such as iodomethane or diazomethane.

Alternatively, mycolic acids can be extracted through methanolic acid hydrolysis. ${ }^{195}$ In this case, a mixture of methanol, sulfuric acid and toluene or methanol/ $6 \mathrm{~N} \mathrm{HCl}$ can be used to release mycolic acid esters which can then be extracted with n-hexane. Both alkaline and acid hydrolysis have been proven effective in preparation of high quality mycolic acid extracts. They also proved to be amenable to scaling down. Few milligrams of cells were satisfactory in obtaining a reproducible HPLC or GC mycolic acid pattern in both cases. $^{196}$

\subsubsection{Lipid Analysis}

After the initial extraction, different bioanalytical methodologies can be applied to create a "lipid profile". The ideal bioanalytical technique to be used for such purpose should result in a lipid profile that would represent the lipid pool of the original sample in both qualitative and quantitative manner. In most cases however, a combination of techniques is required to fully analyze a complex and diverse mixture of lipids such as that of the mycobacterial cell wall. The fact that these lipids represent a wide spectrum of physical properties (different degrees of polarity, ionization potential and extraction accessibility) make it especially challenging for a single technique to overcome this complexity.

The range of techniques that have been used for this purpose included traditional chromatographic techniques (TLC, GC and HPLC), lipid radiolabeling strategies and most recently spectrometric techniques such as MS and NMR. Both spectroscopic techniques have been traditionally reserved for structural elucidation of individual lipid molecules. But with the advancement in both MS and NMR fields and the development of robust statistical methodologies, these two methods are increasingly being applied for comprehensive lipid analysis studies.

Because of their abundance and diversity among different species, mycolic acids received the most attention towards their identification and analysis. Almost the full range of chromatographic techniques has been applied for this purpose. Due to its simplicity and low cost, TLC was the first chromatographic technique to be used in producing mycolic acid patterns. After considerable success and wide application, 
problems of low resolution had to be addressed through the introduction of more sophisticated and powerful chromatographic techniques such as HPLC and capillary GC.

\subsubsection{Simple thin layer chromatography (TLC)}

TLC was the earliest technique used to detect and classify bacteria based on their mycolic acid patterns. When mycolic acids are extracted as mentioned in section 1.7.4. The methyl mycolate esters (usually derivatized by the use of iodomethane) can be separated on thin layer chromatography plates both in one dimension but preferably in two dimensions. ${ }^{185,197}$ The sensitivity of detection can be enhanced by incorporating strategies such as radiolabelling and silver impregnation. ${ }^{198,199}$ Further modification of the solvent system through the addition of small amount of acid has eliminated the need for esterification steps. The most commonly used TLC protocol for mycolic acid analysis now includes two successive developments first in heptane:diethylether:acetic acid 94:5:1 for half length then for the full length in petroleum ether: acetic acid 98:2. ${ }^{198}$

\subsubsection{2D-TLC systems}

Dobson et al introduced the first small scale procedures for comparative analysis of mycobacterial lipids. The methodology was meant to address the full polarity range of mycobacterial lipids by using a sequential solvent extraction to fractionate the loosely bound lipids to polar and non-polar pools. These two lipid pools were then analyzed using five different chromatographical developing systems with increasing solvent polarity. ${ }^{200}$ In the non-polar lipid fraction, two developing systems of low polarity were used to detect triacyl glycerols, menaquinones and phthicerol waxes. By increasing the polarity of the two developing systems, a diverse group of glycolipids were resolved using three other solvent systems with increasing solvent polarity. This group includes acylated trehalose, phenolic glycolipids and sulfolipids. In the polar lipid fraction, glycopeptidelipids, phosphatidylinositol mannosides and other polar glycolipids were resolved using two relatively polar developing systems.

The fact that this method does not rely on specific analytical equipment allowed its wide use by microbiologists. As a result, this method has found application in comparative analysis of different strains, investigating the effect of growth condition in lipid biosynthesis and probing gene functions by providing evidence for inhibition of the biosynthesis of a certain molecule as a result of gene knockout. However it was obvious that the low detection sensitivity allowed the method to be capable only of detecting abundant lipid species and thus it was only useful when gross changes in the lipid pools were expected. An additional step of acidic or alkaline methanolysis is necessary if the method is to be used to analyze the lipid pool of covalently bound fatty acids.

\subsubsection{Gas chromatography $(G C)$}

Gas chromatography has been a major research tool in identification and structural characterization of Mycobacteria cell wall components. The use of capillary GC/MS is

indispensible for the proper identification of the cell wall glycolipids as it is the method 
of choice for identification of the type of the glycol part and the nature of the glycosidic linkage that exist in a molecule. ${ }^{81}$ However, the initial introduction of GC in the mycobacterial research field was only to be used as a tool for identification of Mycobacteria among other microorganisms through pyrolsis. ${ }^{201-203}$ The concept of using $\mathrm{GC}$ in the identification of mycobacterial clinical isolates remains popular among researchers although nowadays it is done through fatty acids pattern rather than pyrolsis products. $^{204,205}$ The diverse repertoire of fatty acids and alcohols that make up the complex cell wall lipids produces a distinct lipid profile that can be used as a finger print to differentiate between species of Mycobacteria. ${ }^{193}$

For this type of analysis, the cellular fatty acid pool is released from the cells through organic solvent extraction and then esterfied through the process of alkaline methanolysis. ${ }^{193,205}$ These methyl esters are then analyzed by capillary GC/MS to produce a specific fatty acid profile that can be used to distinguish between Mycobacteria and other closely related actinomycetes. ${ }^{193,205}$ Due to its accuracy and reproducibility, this protocol has been further developed to include full automation and standardization of the extraction and analytical procedure along with a database that includes fatty acid profiles for most Gram positive and Gram negative bacteria. This fully automated computer assisted identification system is now commercialized under the name Sherlock Microbial Identification System ${ }^{\circledR}$ (SMIS) which is commonly used for simple identification of microorganisms. ${ }^{206}$ Comparison between the automated system and the use of conventional GC analysis of fatty acids methyl esters found that they both produced similar results when used to identify 68 Mycobacteria clinical isolates. ${ }^{205}$

\subsubsection{High performance liquid chromatography (HPLC)}

The center for disease control and prevention (CDC) has incorporated the use of HPLC as a standard procedure for Mycobacteria classification and diagnosis in $1990 .{ }^{187}$ This procedure employs HPLC to produce a well-resolved chromatogram of the cellular mycolic acid pool that is species specific and thus can be used as a diagnostic tool in dealing with mycobacterial infections. After initial steps of saponification followed by acidification and extraction into chloroform, the saponified wax (mycolic acids) are derivatized using p-bromphenacylbromide, 4-bromomethyl-6,7-dimethoxycoumarin, 9anthryldiazomethane or other fluorescent reagent to maximize sensitivity to UV detection. ${ }^{94,207-209}$ These derivatives are then separated by $\mathrm{C}_{18}$-reverse phase column with a methanol/dichloromethane gradient to produce a chromatogram that is unique for a particular species. Two internal standards of different molecular weights are added to aid in the pattern comparisons and recognition, a process that can be achieved visually or through computer-assisted pattern recognitions. ${ }^{187,209}$

The utilization of HPLC for mycolic acid analysis has been modified over a considerable period of time to develop into a protocol that can be highly reproducible and easily automated. Initial problems of long extraction procedures, low sensitivity and subjective process of manual pattern recognition have been successfully overcome. ${ }^{210}$ The result of these refinements is a standard protocol that can decisively identify any of the mycolic acid producing actionmycetes within 40 minutes analysis time and with $98 \%$ 
accuracy. ${ }^{187,211}$ More recently it has been used in some academic setting as a tool to assess the drug susceptibility of certain clinical isolates. ${ }^{212}$

\subsubsection{Mass spectrometry (MS)}

Coupled with GC or HPLC, mass spectrometryhas found great use in the structural determination of many Mycobacteria lipid classes. Since the development of soft ionization techniques such as electrspray ionization (ESI-MS), fast atom bombardment (FAB-MS) and matrix assisted laser desorption ionization (MALDI), MS has been used in sequencing both the peptide and oligosaccharides component of the lipid cell wall. ${ }^{81,102,133,157,213,214}$ With the great advancement in MS, analysis of complex biological mixture without prior separation is now attainable. Soft ionization techniques that produce minimal fragmentation can produce a relatively simple spectrum for a biological mixture. In addition, advancement in resolving techniques and the availability of different analyzers with ultrahigh resolution capability that can reach a value of 100,000 in some cases have offered better accuracy in mass determination that can reach 1ppm.

In a recent example of the utilization of MS in the analysis of mycobacterial lipids, Cox et al has coupled the soft ionization of ESI ion source to the super resolving power of ion cyclotron analyzer to directly analyze a crude mixture of mycobacterial lipids using Fourier transform ion cyclotron resonance (FT-ICR)-MS. ${ }^{215}$ The technique was applicable both to cells grown in vitro and in vivo using ten milliliters of bacterial culture or bacilli isolated from infected mouse lung. The entire free lipid pool was simultaneously monitored and a number of metabolic changes in this pool were identified as a result of gene mutation or limitation in the availability of biosynthetic precursors. With the privilege of having a comprehensive look at the entire lipid pool, the researchers were able for the first time to identify compensatory mechanism and metabolic coupling of two Mycobacteria virulence factors PDIM and SL-1. These metabolic processes were also detected in an in vivo model of mycobacterial infection in mice. Changes in lipid metabolism related to both the relative abundance of the lipid produced and its specific structural modification such as variation in lipid chain length were also detected. ${ }^{215}$ However, some problems were reported by the researchers concerning the inability to recover low abundant lipids after in vivo infection as they were below the limits of detection by this technique. But the relative simplicity of the technique and the wealth of structural information it can offer promises a wide range of future application. A wider application of such method in the mycobacteriology research can be hampered by the limited access to such mass analyzer for most laboratory microbiologists.

Another elegant application of MS in Mycobacteria lipid analysis was proposed by Wenk and colleagues. ${ }^{216}$ In his research, the mass spectrometry analysis of Mycobacteria lipids was preceded by a separation step using HPLC. The HPLC was integrated in that system to allow the analysis of low abundant lipids which may not be detected by MS if analyzed within a mixture of other highly abundant lipids. The ion source chosen was ESI which beside its soft ionization advantages, is better suited to coupling with HPLC. ${ }^{216}$ A potential problem of peak broadening and ion suppression during mass analysis was partially overcome by the use of piperidine in the HPLC mobile phase. The 
addition of piperidine in the mobile phase enhanced ionization and conferred an increase in sensitivity by 2-10 folds. But the choice of relatively high $\mathrm{pH}$ mobile phase necessitated the use of special reverse phase column that can withstand these conditions. An isocratic elution scheme of $15 \mathrm{mM}$ piperidine in a mixture of chloroform: methanol 1:1 was used to effectively separate the mycobacterial lipids over a period of 85 minutes.

The mass analysis of the effluent from the capillary column was achieved using Q-tof mass analyzer operating in the negative mode where most of the membrane and the cell wall phospholipids can effectively ionize. Another advantage of this method is that mycolic acids can be directly analyzed after hydrolysis without the need of a derivatization steps as it is normally required for GC or HPLC-based mycolic acid profiling. The technique was presented as a "non targeted readout" methodology which can be used for the analysis of paired samples (i.e. control versus treated) and was applied by the authors to study the changes in the mycolic acid pool between replicating and dormant $M$. bovis BCG in a qualitative and semi quantitative manner. ${ }^{216}$ The limitation of the method however remains in the low ion response for some groups of non-polar lipids which includes for example acyl glycerols and PDIM.

\subsubsection{Nuclear magnetic resonance (NMR)}

Nuclear magnetic resonance has provided researchers with plethora of structural information about Mycobacteria cell wall components that is unmatched by any other technique. NMR has been an indispensible tool for structural elucidation of all the cell wall components that we came to know. Gross structural features such as the nature of functional groups and the degrees of unsaturation are readily detected by NMR as well as more fine structural features such as the configuration of double bonds and the anomeric carbon of sugar residues. Such information can be obtained with relative ease through analysis of NMR coupling constants. However, NMR use has always been restricted by its relative low sensitivity. This hurdle is likely to be of less significance in the near future with the frequent use of very sensitive sample probe (e.g. cryogenic probe) and the development of smarter pulse sequence. Nevertheless, in the past two decades NMR has found great application in the biological field. For Mycobacteria, an HR-MAS based methodology was developed that uses live bacterial cells to monitor metabolic changes in

polysaccharide components of the cell wall (mainly AG and LAM). ${ }^{189}$ This type of application is likely to remain of limited use in lipid analysis due to irregularities in molecular tumbling and relaxation that can be expected from relatively high molecular weight lipids when dealing with the condition of solid state NMR.

\subsection{Current Challenges in Mycobacteria Research}

Tuberculosis remains the leading cause of death from a bacterial infection. Large efforts have been directed to combat this devastating disease and limit its progression worldwide. Despite these efforts, TB has claimed 1.7 million lives and spread to nine million individuals during the year 2006. ${ }^{12}$ Until the dynamics of the battle between humans and the tubercle bacilli change significantly, it is likely that TB world causalities 
will remain at the same level that we experience today. The first step in eliminating this disease is through new treatment regimens that include an arsenal of drugs with new molecular targets and novel mechanisms of actions. For development of such drugs, researchers in the mycobacteriology field need innovative approaches to achieve a better understanding of this successful pathogen and its challenging capabilities. Some of these challenges are summarized below.

\subsubsection{Mycobacteria Latency and Persistence}

We are aware that during infection Mycobacteria adapt a complex life cycle in which the bacterial population responds to changes in its surrounding environment by shifting between different metabolic states. ${ }^{49,52,53,217,218}$ In establishing a new infection, the bacteria are dividing rapidly inside the human alveolar macrophages in aerobic but acidic condition and remain essentially accessible for chemotherapeutic agents. ${ }^{49}$ However in case of inactive infection, the bacilli remain isolated inside the tubercle structure and so they enter a state of latency (limited cell division) and shift most of the metabolic processes to more of an anerobic nutrient-deprived life style. ${ }^{52,219}$ Due to their isolated location inside the TB granuloma with limited blood supply, halted replication and adaptation to slow metabolic rates, latent bacilli show tolerability to chemotherapy. ${ }^{73,220}$ Upon reactivation and liquefaction of the organized tubercle structure, the bacilli start to rapidly grow and spread to new infection loci as well as to new hosts. ${ }^{49,51}$

Reports have shown that latent bacilli isolated from an intact tubercle are viable but uncultivable in the laboratory under normal growth conditions. ${ }^{52}$ Innovative ways to study the metabolic shifting during the process of latency and subsequent re-activation is necessary to define all elements involved in these processes. A potential success in identifying new molecular targets essential for this process can lead to the emergence of a new class of anti TB drug candidates. Such drugs that abolish Mycobacteria ability to persist in latent state or to be activated to an infectious state will effectively reduce the length of treatment regimens, limit the spread of the infection and significantly reduce the world repertoire of the infectious bacilli.

\subsubsection{Mycobacteria Virulence}

Despite decades devoted to studying virulence determinants of the tubercle bacilli, there has not been a major breakthrough in that field. Our inability to this day to list with clarity the Mycobacteria virulence factors remains frustrating at best despite the availability of complete genome sequences for several virulent and avirulant strains of $M$. tuberculosis. ${ }^{221-224}$

This underscores the need to complete the gene annotation of $M$. tuberculosis genome which possesses many unique genes some of which may have direct involvement in virulence. It is also important to carry out comparative genomics studies aimed at explaining variability in strain virulence among different clinical isolates and different 
lineages. Another aspect concerning Mycobacteria virulence is to identify cellular components that participate in host-pathogen interactions and to define signaling pathways that mediate cell entry and promote bacteria survival inside macrophages. Research done so far in this area has implicated several cell wall lipids such as PIM and manLAM in triggering the host immune response and halting the process of phagosomal maturation respectively. ${ }^{66,110,111}$

\subsubsection{Mycobacteria Drug Resistance}

Tuberculosis is no longer the incurable epidemic (white plague) it once was which claimed millions of life in Europe in the $19^{\text {th }}$ century. ${ }^{20} \mathrm{We}$ owe this to the development of several anti-TB drugs starting with streptomycin in $1944 .{ }^{21}$ However, the current regimen for TB treatment remains exceptionally long (the short treatment course last for six months and involves four drugs), which results in poor adherence of TB patients to that long and seemingly unnecessary course. ${ }^{54,55}$ When a patient stops his treatment improperly due to relief of symptoms, the bacterial growth is only partially suppressed allowing the enrichment of the bacterial population with naturally occurring resistant mutants. Once the resistant strains are activated again, the infection is exceptionally hard to treat and typically requires two years of therapy with the possibility of spreading the infection to others during this period. ${ }^{34}$ MDR-TB now has a global distribution while the more serious XDR-TB is being reported in several countries including the United States. ${ }^{15,34,36}$ Several questions remain on the best way to curb the spread of such dangerous strains as well as to the molecular basis to this sort of multiple drug resistance.

\subsubsection{NTM Infections and Emerging Mycobacterial Diseases}

The incidence of non tuberculous Mycobacteria (NTM) lung disease is alarmingly on the rise. ${ }^{15}$ The term NTM is generally used to describe Mycobacteria species other than that of the M. tuberculosis complex and M. leprae. ${ }^{4}$ These NTM are ubiquitous environmental organisms that we are exposed to everyday as they exist in the soil and water. ${ }^{2,225}$ M. avium has been known to have the ability to colonize parts of the human body such as the urinary and respiratory tract without causing diseases. ${ }^{16}$ However, individuals infected with HIV are especially vulnerable to infection with these organisms due to their severely compromised immune system. The most prevalent NTM infections among HIV patients are the disseminated infection with members of M. avium complex or MAC. ${ }^{226}$ Most recently, cases of NTM lung disease are being reported in several western countries in individuals with no history of immune-suppression or HIV infections. ${ }^{16,227}$ We have limited understanding of the pathogencity of these bacteria and the disposing factors (other than HIV) that can make an individual vulnerable to these infections. All members of MAC complex produce highly antigenic glycopeptidolipids GPL but until now these fascinating molecules have not been shown to play a specific role in virulence and pathogenicity. ${ }^{155}$ Other less common NTM do not make these haptens but are still capable of producing serious infections in some individuals. ${ }^{228-230}$ More research is necessary to define the molecular basis underlying the infectivity of 
NTM and the host pathogen interaction associated with them. Such effort are likely to reveal interesting new pathways in which Mycobacteria surface components can interact with the host cells and how these events affect the clinical outcome.

In certain areas of the globe, there is a growing number of cases of infections with another mycobacterium species. Buruli ulcer (BU), caused by M. ulcerans, is now reported in more than thirty countries with the highest incidence rate in West Africa. ${ }^{14,38}$ Once not recognized by physicians and scientists, BU infections have been emerging rapidly as a serious health concern during the 1980s. In response to the emergence of this disease, WHO has launched the Global Buruli Ulcer Initiative in 1998 to raise awareness of the disease and encourage scientific research to further define the disease and its causative agent and help find new treatment options for the thousands affected by it.

Due to the prevalence of BU in relatively poor and remote communities, the exact incidence rate of $\mathrm{BU}$ is not completely known. Also, prevention strategies are hard to implement since the mode of transmission of M. ulcerans has not been established yet. Beside our need to understand disease transmission and pathology, there is an urgent demand to develop new treatments and vaccines to counteract the emergence of such devastating illness. One of the remarkable breakthroughs in our understanding of BU was the establishment of M. ulcerans virulence factors. In 1999, George, KM et al reported the identification and chemical characterization of mycolactone $\mathrm{A} / \mathrm{B}$, a macrolide toxin secreted by $M$. ulcerans that produced the same pathological features as BU in animal models. $^{231}$

\subsection{Purpose of This Study}

Our understanding of the mycobacterial cell wall, its biosynthesis, structural features and the role each cell component plays in the pathogenicity is largely based on data obtained through multivariate experiments that evaluate a single molecule at a time. A better way to study the mycobacterial cells should include a global approach to provide a panoramic view of as many metabolites as possible simultaneously and avoid the drawbacks of dissecting cell metabolites on multiple experiments. The key focus of the research project discussed in this dissertation is to utilize the analytical power of NMR spectroscopy to develop a new methodology for global analysis of Mycobacteria lipid pool as a complementary technique to the previously developed HR-MAS that focuses mainly on the cell wall polysaccharides. The two techniques share a similar strategy of

${ }^{13} \mathrm{C}$-isotope enrichment and reliance on ${ }^{1} \mathrm{H}-{ }^{13} \mathrm{C} 2 \mathrm{D}-\mathrm{HSQC}$ experiments. When combined together the two techniques will provide a comprehensive analysis of major cell wall components in reasonably short time period and with minimal sample manipulation.

The goals of this research were outlined to insure the simplicity of the new methodology which will make it available to a wide spectrum of investigators. The results obtained from such techniques have to be easily analyzed and standardized to allow valid comparison of results obtained by different research groups. The following 
chapters offer a thorough discussion of the experimental design and the results obtained towards achieving the main goals of this research which include

- Development of a lipid profiling technique with an optimized extraction, acquisition and analysis protocols that insure the reproducibility of the results.

- Validation of the methodology in providing unbiased representation of the cellular lipid pools of several Mycobacteria species.

- Combination of the method with HR-MAS technique for qualitative and quantitative monitoring of adaptive changes in the cell wall metabolites when cells are grown under stress conditions.

- Evaluation and application of MS and NMR-based techniques in targeted as well as non-targeted lipid analysis. 


\section{CHAPTER 2. DEVELOPMENT OF A NEW METHOD FOR ANALYSIS OF MYCOBACTERIA LIPID POOL ${ }^{1}$}

\subsection{Introduction}

The cell wall of Mycobacteria has a very complex and unique structure with notably high lipid content. Great research efforts have been directed to the characterization of the individual components of the cell wall and investigating the role of these components in Mycobacteria pathogenicity and virulence. Early attempts for global analysis of the mycobacterial lipidome began in the 1960s during the search for a proper taxonomical tool to assign newly discovered species in their right taxonomical order and to help identify clinical isolates to the species level. ${ }^{86,233,234}$ A major breakthrough in this field was achieved by Dobson and Minnikin who reported five 2D-TLC systems in the 1980s to analyze the mycobacterial lipidome. ${ }^{200}$ Despite its wide application, this methodology remained less than satisfactory. Problems associated with poor staining, low sensitivity and limited resolution on one hand and the recent advancement of bioanalytical tools on the other, have fueled a research effort for new methodologies to study the mycobacterial lipidome.

Recently, two new methodologies based on mass spectrometry have been described. The first uses Fourier transform ion cyclotron resonance mass spectrometry (FT-ICR)MS. $^{215}$ The second uses prior separation with HPLC followed by analysis with electrospray ionization HPLC/ESI-MS. ${ }^{216}$ These analytical techniques are highly sensitive, however, they require significant sample manipulation to overcome the phenomena of ion suppression within the lipid mixtures thus are best used for the analysis of readily ionizable molecules. ${ }^{215,216}$

All these methodologies offer great tools to investigate adaptive changes in mycobacterial lipid pools. Such techniques can be used to study the mode of action of anti-tuberculosis drugs, determine gene function, understand the stringent response of Mycobacteria under stress conditions that resemble its in vivo environment, and to investigate differences between species or strains of Mycobacteria. To maximize their benefit, these lipidomics techniques are best used in complementary fashion and together with other genomics and proteomic methods. As it is the case with other "omics" techniques, the great utility of these methods is that they provide a wealth of information regarding the total composition of the lipid pools, from which a small number of candidate molecules can then be defined for more comprehensive follow up studies through purification and isolation of such molecules of interests.

The method being developed in this dissertation focuses on the application of NMR spectroscopy as a complementary tool that can be utilized to rapidly analyze the pool of

\footnotetext{
${ }^{1}$ Parts of this chapter are adapted with permission. Mahrous, E.A., Lee, R.E. \& Lee, R.E. Lipid profiling using two dimensional heteronuclear single quantum coherence NMR. in Lipidomics: Methods and Protocols (Humana Press, New York, 2009). In press. ${ }^{232}$
} 
membrane and surface free lipids directly. Considered as a universal technique, the use of NMR can be successful in certain areas where other analytical methods have failed. As NMR measures properties of nuclei rather than the physical property of the entire molecule, NMR signals are less likely to be affected by the different physicochemical properties exhibited by different classes of lipids (Molecular weight, polarity, ionization potential, etc). This uniformity of response is advantageous because it limits artifacts and confers quantitative representation of all lipids regardless of their structures. However, several challenges exist for using NMR to study complex pools of metabolites. The first of which is the low sensitivity associated with NMR analysis. This can be partially overcome by enriching the cell lipidome with ${ }^{13} \mathrm{C}$-isotope through the replacement of the carbon source in the growth media with ${ }^{13} \mathrm{C}$-labeled lipid precursors. The second challenge is the complexity and the frequent overlapping of signals in the NMR spectrum. This problem can be addressed through the use of two and three dimensional NMR. Two dimensional ${ }^{1} \mathrm{H}_{-}{ }^{13} \mathrm{C}$ heteronuclear single quantum coherence (2D-HSQC) NMR can be used to rapidly generate lipid profiles with acceptable resolution, while more sophisticated three dimensional experiments can be used to resolve the lipid profile and help assign signals in the 2D-HSQC spectrum when necessary.

Beside the choice of the appropriate NMR pulse sequence, analysis of such complex data requires extra consideration and efforts. To insure data quality, signals in the NMR spectra need to be referenced in both their chemical shift and relative intensity to an internal reference that produces a discrete signal in the 2D-HSQC NMR spectrum. To enhance reproducibility, external references may be required to calibrate the NMR spectrometer and account for any observed variation due to instability of the high frequency magnet over time. The data must then be normalized based on these internal and external standards using suitable software which confers robust analysis of large data sets.

Other than NMR acquisition criteria, several parameters regarding cell growth conditions and extraction procedures can also contribute to the quality of the lipid profiling process. The extent of ${ }^{13} \mathrm{C}$-enrichment is controlled by several factors including the type of growth media, size and strategy of inoculation, the concentration and the nature of ${ }^{13} \mathrm{C}$-sources used. On the other hand, extraction procedures can be affected by the type of extraction solvent, temperature and duration of extraction and the ratio between cell weight and the volume of solvent used. Hence, several manipulations within the growth and extraction strategy can be sought to target the analysis of specific subpopulation.

\subsection{Materials and Methods}

\subsubsection{Strains and Growth Conditions}

M. smegmatis and M. bovis were grown in liquid growth media at $36.5^{\circ} \mathrm{C}$ with continuous shaking at $250 \mathrm{rpm}$. Middlebrook $7 \mathrm{H} 9$ was used for growing the bacilli after 
addition of $10 \%$ ADG supplement and $0.05 \%$ Tween- 80 . For ${ }^{13} \mathrm{C}$-enrichment, the main carbon source (dextrose and glycerol) were replaced with ${ }^{13} \mathrm{C}_{6}$-dextrose and ${ }^{13} \mathrm{C}_{3}$-glycerol respectively. For method development, $M$. smegmatis $\mathrm{mc}^{2} 155$ and $M$. bovis BCG were used as surrogate for M. tuberculosis. M. smegmatis was inoculated at a starting O. $\mathrm{D}_{\lambda 600}$ of 0.005 while $M$. bovis BCG was inoculated at starting $\mathrm{O}_{2} \mathrm{D}_{\lambda 600}$ of 0.05 .

\subsubsection{Total Lipid Extraction and Fractionation}

Cell cultures were harvested by centrifugation at $3700 \mathrm{xg}$ for 10 minutes at room temperature. The cell pellet was washed twice with 5 milliliters of deuterium oxide $\left(\mathrm{D}_{2} \mathrm{O}\right)$ and the supernatant was removed by repetitive centrifugation and decantation in between. One milliliter of $\mathrm{D}_{2} \mathrm{O}$ was used to transfer the washed cell pellet to a pre-weighed microcentrifuge tube. The cell suspension was then centrifuged at 21,000xg for ten minutes and all remaining $\mathrm{D}_{2} \mathrm{O}$ was carefully removed. For total lipid extraction, a 2:1 mixture of $\mathrm{CDCl}_{3}: \mathrm{CD}_{3} \mathrm{OD}$ was added to the washed cell pellet $(3 \mu \mathrm{l}$ per $1 \mathrm{mg})$ of wet cell weight. DMSO was added in certain experiment to be used as an internal standard to a final concentration of 4,8 and $16 \mathrm{mM}$. The extraction was achieved at $37^{\circ} \mathrm{C}$ with continuous shaking for 20, 40, 90 and 120 minutes.

For fractionation of the cellular lipid into polar and non polar fraction, two layers were separated by direct centrifugation of the cell suspension at 21,000xg for 10 minutes. The aqueous upper layer (polar lipids) and the organic lower layer (apolar lipid) were separately transferred to 5mm NMR tube for analysis. For analysis of the total lipidome, extra $\mathrm{CD}_{3} \mathrm{OD}$ was added to the extraction mixture $(1 \mu \mathrm{l}$ per $1 \mathrm{mg}$ of cells) to bring it to a single phase solution. Then the extract was separated from the cell debris by centrifugation as above. In some instances, other extraction solvents were also used such as acetone- $\mathrm{d}_{6}$ and benzene- $\mathrm{d}_{6}$.

\subsubsection{Mycolic Acid Extraction}

Delipidated cell pellets were re-suspended in two mls of $1 \mathrm{M}$ methanolic $\mathrm{KOH}$. The cell suspension was heated in a screw capped glass tubes at $70^{\circ} \mathrm{C}$ for 16 hours with continuous stirring. After the alkaline hydrolysis, the extract was cooled down and neutralized with concentrated $\mathrm{HCl}$ to release free mycolic acids. The extract was then diluted with four volumes of deionzied water before being extracted twice with one volume of diethyl ether. The combined ether extract was washed with deionized water and brought to dryness under vacuum using a rota-evaporator at $40^{\circ} \mathrm{C}$. For NMR analysis, the mycolic acid residue was dissolved in a 1:1 mixture of $\mathrm{CD}_{3} \mathrm{OD}: \mathrm{CDCl}_{3}$.

\subsubsection{NMR Acquisition and Data Processing}

All NMR experiments were done using Varian-Inova 500MHz spectrometer equipped with a $5 \mathrm{~mm}$ triple resonance trpfg probe. $2 \mathrm{D}$-HSQC pulse sequences were done at $24^{\circ} \mathrm{C}$ 
for 256 increments and 2, 4, 12 or 60 transients after each sample was tuned, shimmed and locked separately. Other acquisition parameters were optimized for each sample as well. Felix-NMR 2007 were used to process and analyze the data after referencing to the DMSO peak at $\delta^{1} \mathrm{H}=2.67, \delta^{13} \mathrm{C}=39.4 \mathrm{ppm}$.

\subsection{Results}

\subsubsection{Production of NMR Profiles and Assigning Signals of Some Cell Wall Lipids}

The isotope enrichment strategy greatly enhanced the signal intensity and conferred a good spectrum in a relatively short acquisition time. A good quality 2D spectrum was attainable in 20 or 40 minutes. However, observation of some less abundant lipid species necessitated a longer acquisition time of 90 minutes (Figure A.1). Signals in the HSQC spectrum were well dispersed and can be categorized into three main regions; the most up field region $\left(\delta^{1} \mathrm{H},-0.5-3.0 \mathrm{ppm}\right)$ representing the aliphatic chain of the lipid molecules, the far down field region $\left(\delta^{1} \mathrm{H}, 5.2-8.5 \mathrm{ppm}\right)$ representing unsaturated and aromatic substructures and the middle region $\left(\delta^{1} \mathrm{H}, 3.0-5.4 \mathrm{ppm}\right)$ which was crowded with signals mainly from the sugar residues of different glycolipids (Figure 2.1). Although there is significant crowding of some signal areas of the HSQC spectrum, since every molecule is represented by multiple signals, we found that at least one or two signals from each lipid molecules of interest did not overlap with other signals (examples are shown in Figure 2.1). Therefore, the presence or absence and the relative quantity of a certain molecule can be readily determined by these diagnostic biomarker peaks (Table 2.1). When possible, it is useful to identify and utilize multiple biomarker signals for a certain molecule of interest to confer a greater degree of confidence in the data interpretation and hence limit the possibility of artifacts from unidentified overlapping species.

\subsubsection{Effect of Different Growth Conditions}

It has been reported that the composition of the cellular lipid pool can change depending on the growth conditions including the type of carbon sources used in the growth media. ${ }^{158,159}$ Glycerol grown Mycobacteria usually accumulate large amounts of acylated glycerols, while cells that depend in their growth on glucose as the main carbon source tend to form less acylated glycerol and more of the acylated hexoses. ${ }^{159,235} \mathrm{We}$ observed similar changes in the growth of $M$. smegmatis in Middlebrook 7H9 media when the growth media was supplemented with either ${ }^{13} \mathrm{C}_{6}$-dextrose or ${ }^{13} \mathrm{C}_{3}$-glycerol as shown in Figure 2.2.

Some differences were also observed between the lipid profiles of cells growing in liquid media, Middlebrook 7H9, and that growing in a parallel solid media, Middlebrook $7 \mathrm{H} 11$ agar. In the case of M. bovis BCG, we reported a general increase in the production of triacyl glycerols, phenolic glycolipids (mycoside B) and methyl-branched fatty acids in cells grown on solid media (Figure 2.3). On the other hand, the production of 


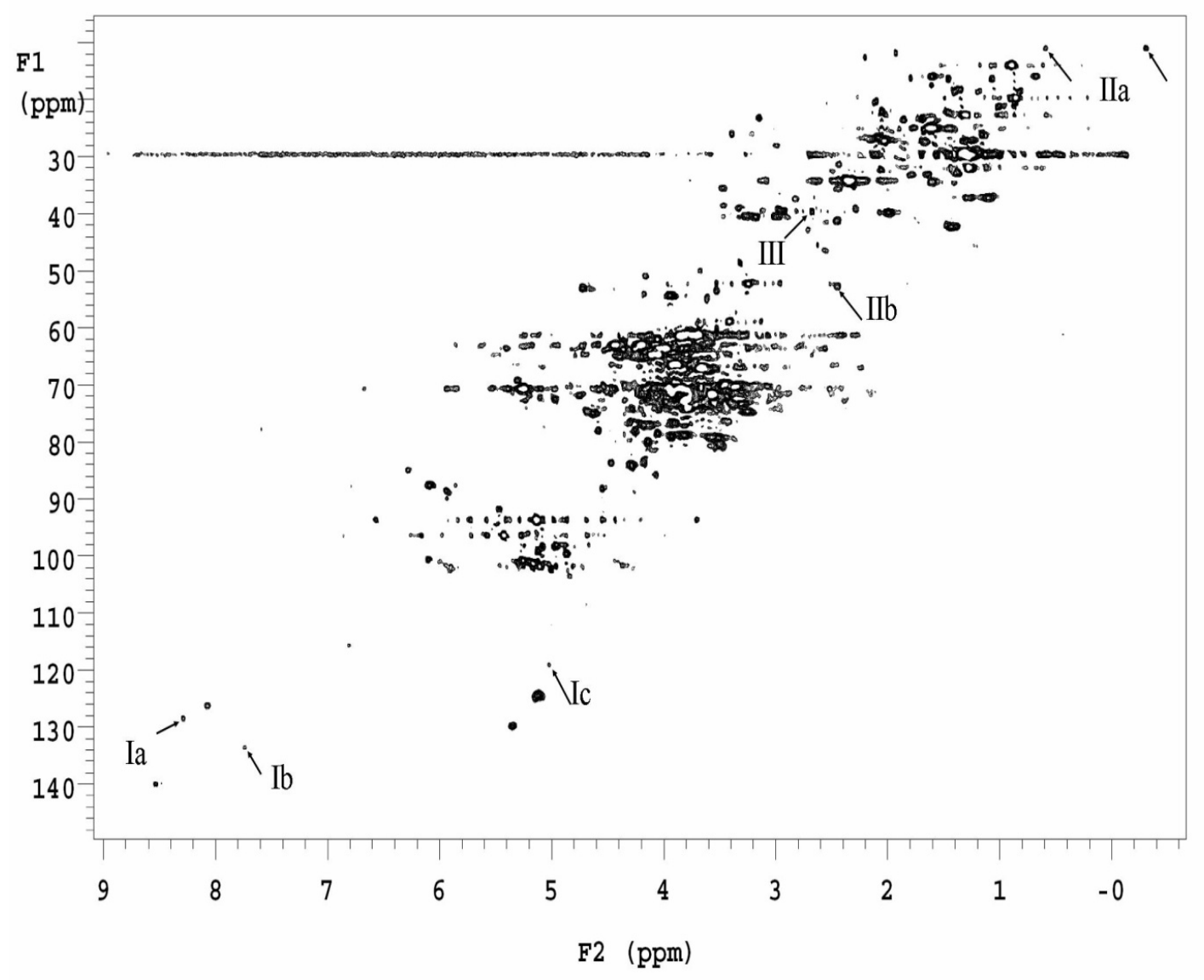

Figure 2.1 2D-HSQC Lipid Profile of M. bovis BCG

${ }^{1} \mathrm{H}-{ }^{13} \mathrm{C}$ HSQC spectrum of the $2: 1 \mathrm{v} / \mathrm{v} \mathrm{CDCl} 3: \mathrm{CD}_{3} \mathrm{OD}$ extract of $M$. bovis $\mathrm{BCG}$. The extract was obtained as described in the text (section 2.2.2). For illustration, three biomarker signals for menaquinones are shown (signals Ia-c), and two biomarker signals are shown for trehalose dimycolate (signals IIa,b) including the diastereotopic $\mathrm{CH}_{2}$ of the cis cyclopropyl ring. Signal III indicates the signal for DMSO, which was included as an internal standard during the extraction. The total sample preparation time including extraction was two hours. Total NMR acquisition time was 1 hour and 40 minutes $(\mathrm{ni}=256, \mathrm{nt}=10)$. 
Table 2.1 Chemical Shift Values of Major Lipid Biomarkers

\begin{tabular}{|c|c|c|c|c|c|}
\hline Description & $\begin{array}{l}\delta^{1} \mathbf{H} \\
\text { ppm }\end{array}$ & $\begin{array}{l}\delta^{13} \mathrm{C} \\
\mathrm{ppm}\end{array}$ & Description & $\begin{array}{l}\delta^{1} \mathrm{H} \\
\mathrm{ppm}\end{array}$ & $\begin{array}{l}\delta^{13} \mathrm{C} \\
\text { ppm }\end{array}$ \\
\hline $\mathrm{TAG}^{\mathrm{a}}$ & & & Mycobactins $^{b}$ & & \\
\hline CH-glycerol & 5.30 & 69.31 & aromatic $\mathrm{CH}$ & 7.15 & 130.73 \\
\hline $\mathrm{CH}_{2}$-glycerol & $\begin{array}{l}4.38, \\
4.19\end{array}$ & 62.43 & aromatic $\mathrm{CH}$ & 6.81 & 115.86 \\
\hline 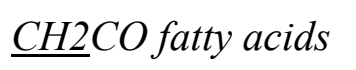 & 2.35 & 34.18 & $\mathrm{PDIM}^{\mathrm{c}}$ & & \\
\hline acylated trehalose $\mathrm{e}^{\mathrm{a}}$ & & & $\underline{\mathrm{CH}}_{2}=\mathrm{CH}-\mathrm{COOR}$ & $\begin{array}{l}0.96 \\
1.23\end{array}$ & 45.54 \\
\hline trehalose- $\mathrm{C} 6 \mathrm{CH}_{2}$ & $\begin{array}{l}4.21, \\
4.44\end{array}$ & 63.08 & $\mathrm{CH}_{2}-\underline{\mathrm{CH}}-\mathrm{COOR}$ & 4.87 & 71.55 \\
\hline trehalose- anomeric & 5.13 & 93.65 & $\begin{array}{l}\text { Methylated fatty } \\
\text { acids }^{\mathrm{c}}\end{array}$ & & \\
\hline Fatty acids ${ }^{\mathrm{a}}$ & & & $-\mathrm{CH}_{3}$ & 1.05 & 17.37 \\
\hline cis double bond & 5.34 & 129.97 & $-\mathrm{CH}_{3}$ & 1.16 & 19.69 \\
\hline trans double bond & 5.12 & 124.62 & Phospholipids ${ }^{\mathrm{b}}$ & & \\
\hline $\mathrm{GPL}^{a}$ & & & CH-glycerol & 5.26 & 70.47 \\
\hline phenyl alanine & 7.27 & 128.71 & $\mathrm{PIM}_{2}$ anomeric & 5.10 & 101.85 \\
\hline methoxy sugars & 4.67 & 55.36 & PIM $_{6}$ anomeric & 5.01 & 102.63 \\
\hline Menaquinone $^{\mathrm{b}}$ & & & $\mathrm{TDM}^{\mathrm{b}}$ & & \\
\hline aromatic ring & 7.74 & 133.64 & $\underline{\mathrm{CHCO}}$ & 2.47 & 52.81 \\
\hline double bond & 5.05 & 118.94 & Cyclopropyl $\mathrm{CH}_{2}$ & $\begin{array}{l}-.33, \\
0.58\end{array}$ & 10.73 \\
\hline
\end{tabular}

${ }^{\text {a,b,c }}$ Chemical shifts were inferred from 2D-HSQC spectra of M. smegmatis, M. bovis or M. ulcerans respectively. Subtle variations in these chemical shifts can be observed between species due to minor structural details. 

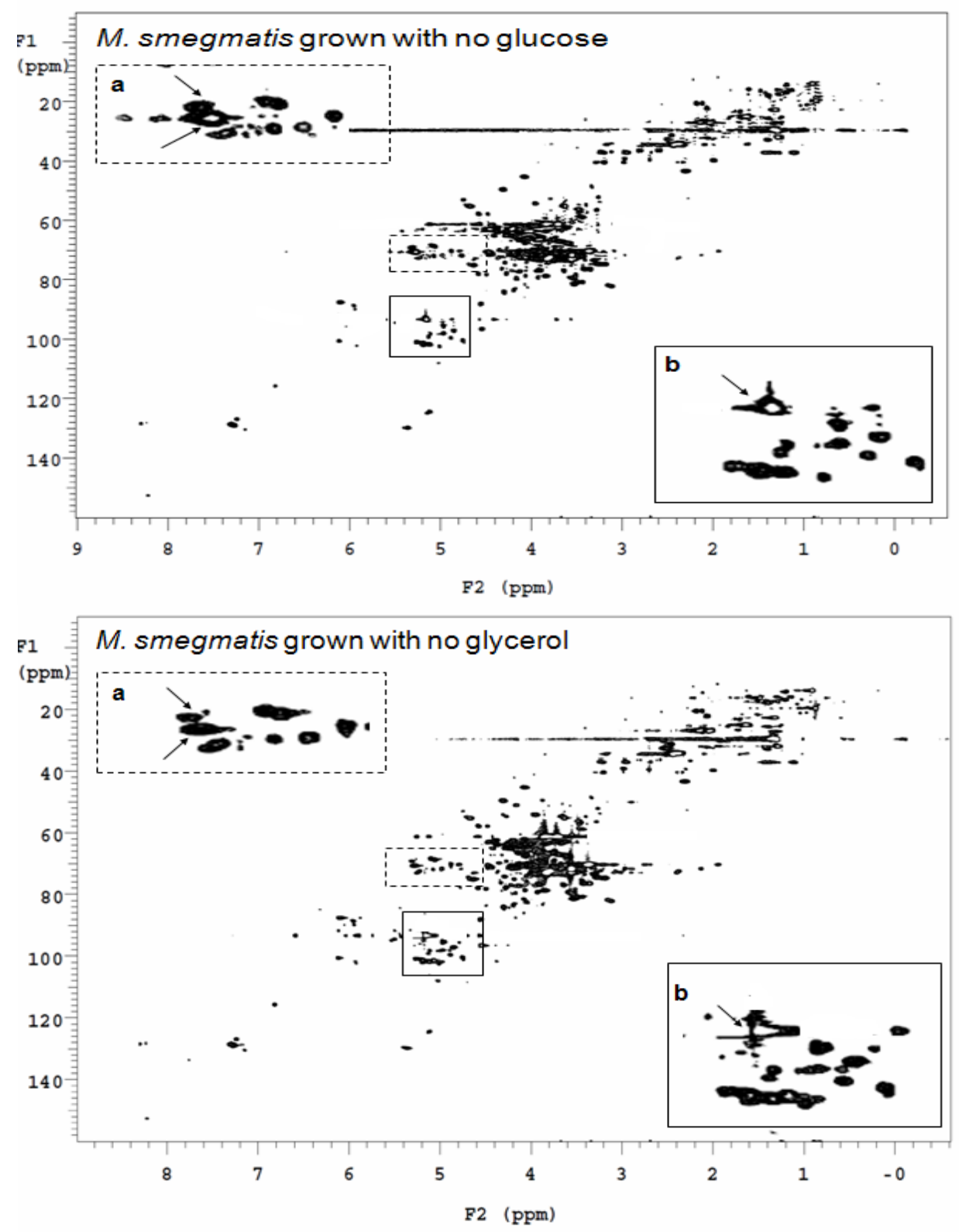

Figure 2.2 Effect of the Composition of Growth Media on the Lipid Profile of $M$. smegmatis

Insert (a) is an expanded window of the area where the signals of different triacyl glycerols and glycerol phospholipids are usually detected. Signals of TAG and phosphatidyl inositol, indicated by arrows, were recorded at lower intensity in the absence of glycerol from the growth media (lower spectrum). Insert (b) is an expanded window of the area where the anomeric signals of acylated hexoses are usually recorded. These signals were recorded at lower intensity in the absence of dextrose in the media (upper spectrum). The anomeric signal of acylated trehalose is indicated by an arrow. 

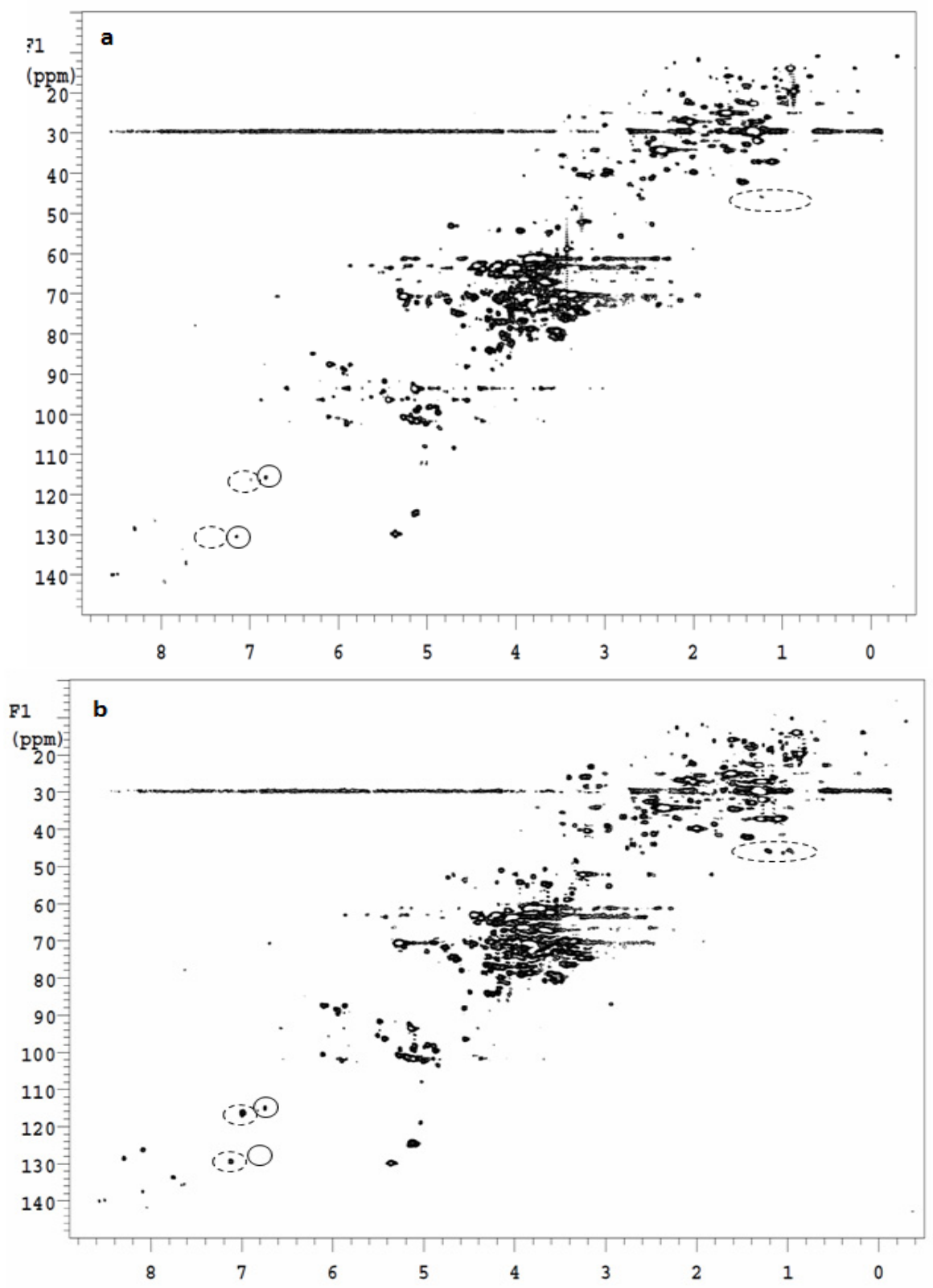

Figure 2.3 Effect of the Use of Different Growth Media on the Lipid Profile of M. bovis BCG

M. bovis BCG cells were harvested and extracted from either ${ }^{13} \mathrm{C}$-labeled $20 \mathrm{mls}$ culture, (a), or the lawn of one ${ }^{13} \mathrm{C}$-labeled 7H11 agar plate, (b). Signals of mycoside B were detected at higher intensity in the lipid profile of cells grown on solid media (biomarkers of mycoside B are enclosed in dashed-line circles). However, mycobactins were detected at higher intensity in the lipid profile of cells grown in liquid media (biomarkers of mycobactins are enclosed in solid-line circles). Effect of using different growth media in the lipid profile of M. avium is shown in Figure A.2. 
mycobactins seemed to be of less abundance in cells grown on solid media. These differences can be either due to a real metabolic shift as a result of a change in the growth environment of the bacteria or due to shedding off some surface lipids in the liquid growth media with the continuous shaking and the addition of a surfactant such as Tween-80.

Cells have also been extracted at different growth stages including early exponential phase, mid exponential phase and stationary growth phase. The rapid cell division and turnover of metabolites in the exponential phase was translated to relatively low signal intensities in the lipid profiles of cells growing at optical density of 0.3- 0.4 (Figures 2.4a, A3). Cells harvested at later stages of growth at optical density of $\geq 0.7$ demonstrated more diversity and intensity of lipid signals especially in the areas where glycolipids signals are usually detected (Figures 2.4b, A4). However, throughout the different stages of growth, stability in the relative abundance of glycerolphospholipids can be observed. This class of lipids is indispensable for the integrity of the cell membrane of living cell and so they may be used as an indicator of the overall spectral quality or as an additional internal standard when two sets of spectra are being compared.

In principle, supplementing the growth media with specific ${ }^{13} \mathrm{C}$-labeled lipid precursors can achieve some selective labeling of certain group of lipids. For example, adding ${ }^{13} \mathrm{C}_{2}$-sodium acetate in the growth media of the cells resulted in selective labeling of straight chain fatty acids and significant reduction in the intensity of glycolipids signals and signals pertaining to polyketide lipids such as PDIM. The result was a 2D-HSQC spectrum that was representative mainly of straight chain fatty acid substituents of major cell wall lipids (Figures 2.5, A.5).

\subsubsection{Effect of Initial Culture Volume, Solvent and Duration of Extraction}

The choice of the extraction solvent to be used depends mainly on the pre-determined target of the lipid profiling. In certain cases, it may be desired to profile a specific sub population like apolar lipids, acetone soluble lipids thus the extraction solvent will be chosen accordingly. For total lipid analysis, a 2:1 mixture of chloroform- $\mathrm{d}_{1}$ and methanol$\mathrm{d}_{4}$ is regarded as a first choice due to the existence of validated well-respected lipid extraction protocols by both Folch ${ }^{191}$ and Bligh and Dyer ${ }^{192}$ that depend on the use of chloroform:methanol mixtures.

Cells harvested from 20 and 50 milliliters cultures and extracted with 2:1 mixture of $\mathrm{CDCl}_{3}: \mathrm{CD}_{3} \mathrm{OD}$ to a final volume of $500-600 \mu$ produced good quality $2 \mathrm{D}-\mathrm{HSQC}$ spectra using $5 \mathrm{~mm}$ trpfg probe (Figure A.6). On the other hand, cells harvested from smaller 10 milliliters culture $(50 \mathrm{mg})$ required the use of $3 \mathrm{~mm}$ NMR probe to accommodate smaller sample volumes of about $150 \mu \mathrm{l}$ to produce a $2 \mathrm{D}-\mathrm{HSQC}$ spectrum of comparable quality and in reasonable acquisition time (Figure A.7).

Extraction with $\mathrm{CDCl}_{3}: \mathrm{CD}_{3} \mathrm{OD}$ for 20 minutes was sufficient to produce a good quality lipid profile of the major cellular lipids. Longer extraction time of 40, 90 and 120 

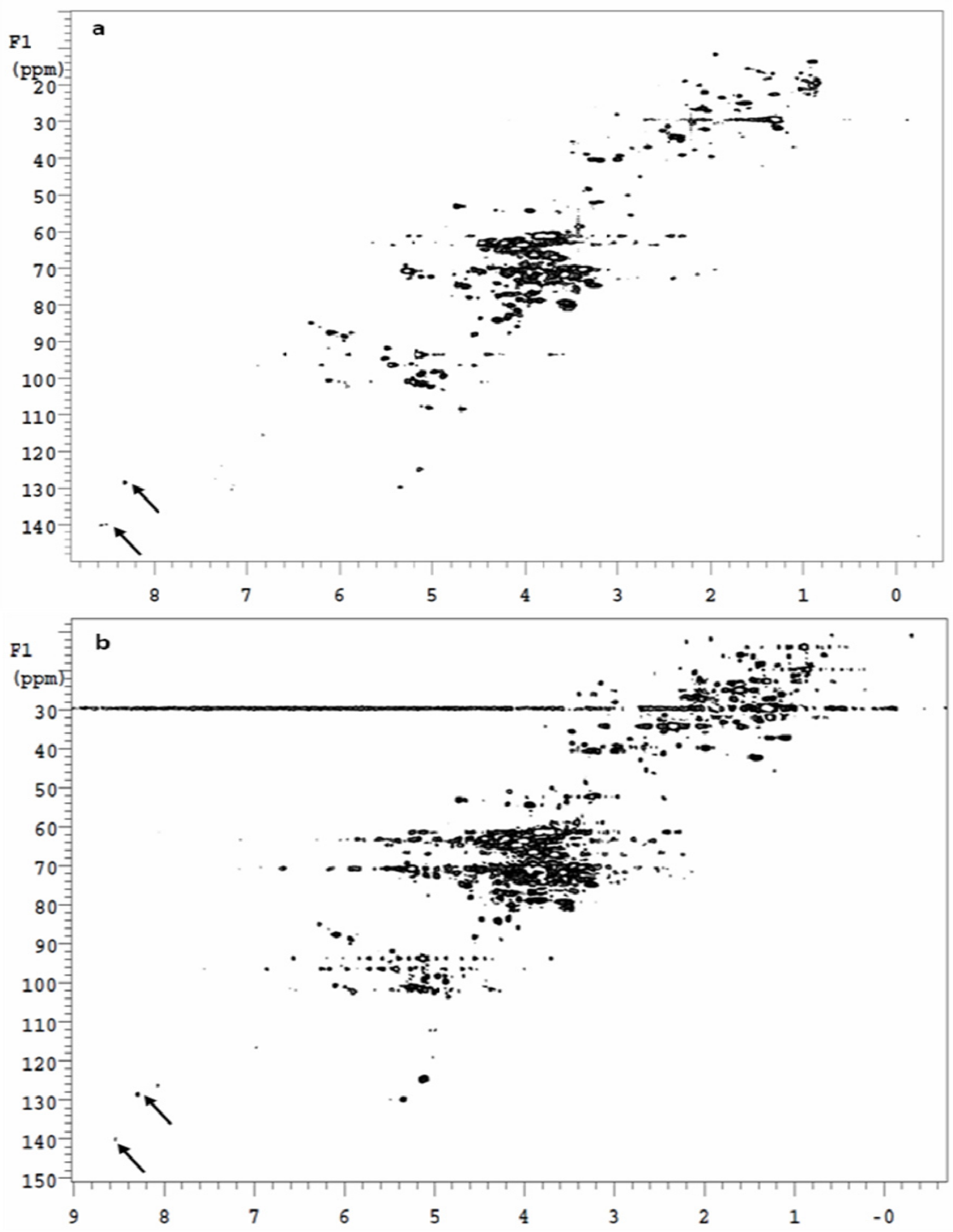

Figure 2.4 Lipid Profile of M. bovis BCG Harvested at Different Growth Stages

M. bovis BCG cells were harvested and extracted at different stages of growth; at O.D $\mathrm{D}_{\lambda 60}=0.4$, (a), and O.D $\lambda_{600}=0.72$, (b). Lipid signals were generally reported at higher intensity in cells harvested at later stages of growth. The two spectra were normalized in their intensities to the intensity of menaquinones signals, indicated by the small arrows. Lipid profiles of $M$. bovis BCG cells harvested at other optical densities are shown in Figures A.3, A.4. 

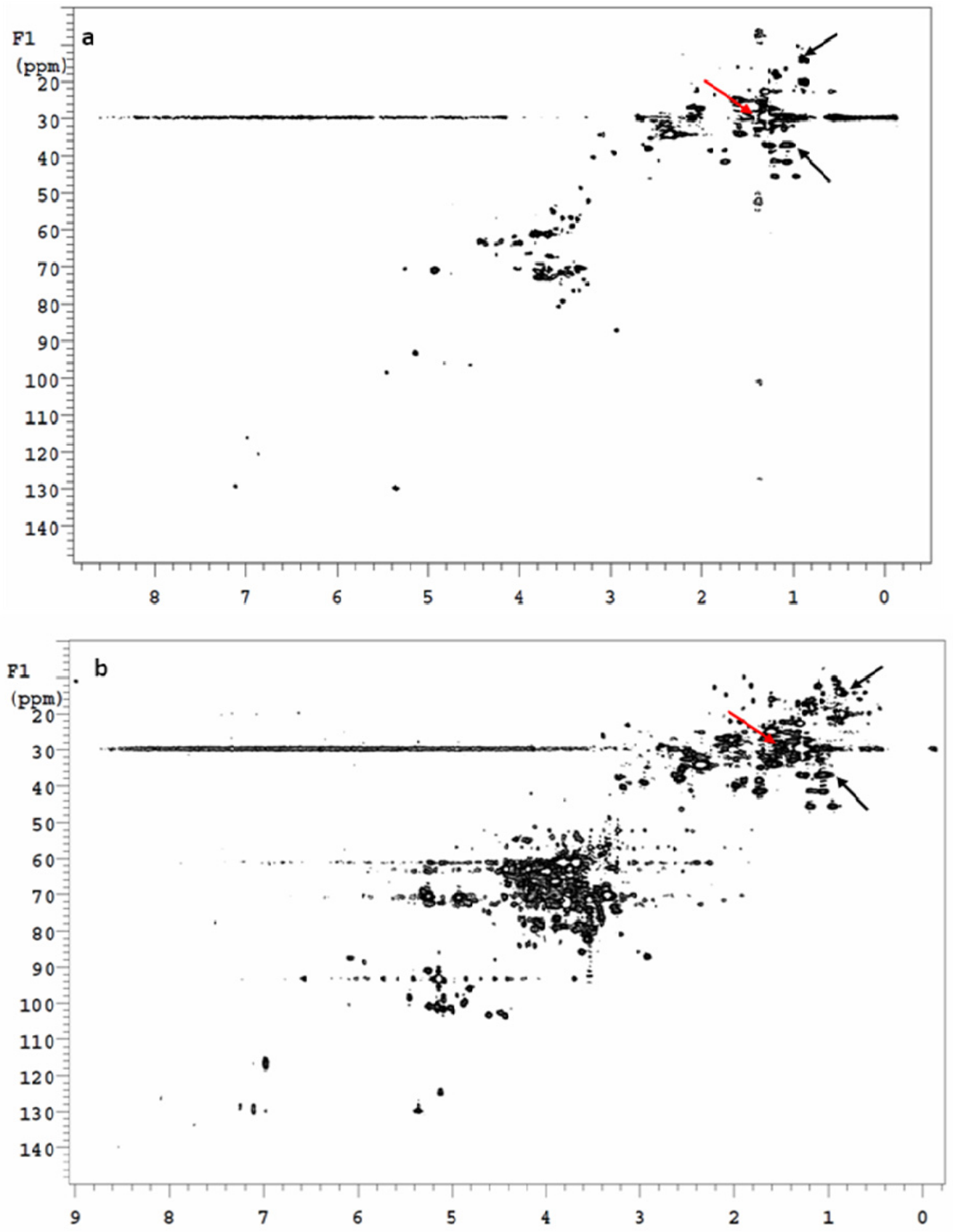

Figure 2.5 Effect of Acetate Labeling on the Lipid Profile of M. marinum

Cells were grown in Middlebrook $7 \mathrm{H} 9$ where $0.2 \%{ }^{13} \mathrm{C}_{2}$-sodium acetate have been added as a ${ }^{13} \mathrm{C}$-source, (a), or ${ }^{13} \mathrm{C}_{6}$-dextrose and ${ }^{13} \mathrm{C}_{3}$-glycerol were used as ${ }^{13} \mathrm{C}$-source, (b).

Acetate labeling resulted in selective labeling of straight chain fatty acids as indicated by the presence of fatty acids signals at comparable intensity in the two spectra (representative signals for straight chain fatty acids are indicated by arrows). Another example for the effect of acetate labeling is shown in Figure A.5. 
minutes offered better representation of lipids of lower abundance with no substantial improvement observed for extraction longer than 90 minutes (Figure A.8). In all four cases, the lipid profile produced after re-extraction of the pellet was of poor quality and only displayed signals of some fatty acids and some membrane phospholipids (Figure 2.6).

Extraction with acetone- $\mathrm{d}_{6}$, produced good quality spectra but with a slight bias towards non polar lipids and against glycolipids, especially phospholipids (Figures 2.7, A.9). Other solvents with lower polarity such as benzene and petroleum ether failed to produce a reasonable quality spectrum when used to extract the $\mathrm{D}_{2} \mathrm{O}$ washed cell pellets. In fact, re-extraction of the cell pellet remaining after extraction with benzene- $\mathrm{d}_{6}$ produced a lipid profile where signal intensities were comparable to other profiles obtained with a single step chloroform: methanol extraction (Figure A.10). Due to its low dielectric constant, benzene- $\mathrm{d}_{6}$ as a solvent failed to solubilize the relatively polar cell wall glycolipids that are included within an organized mycolic acids layer in the outer membrane.

\subsubsection{The Use of DMSO as an Internal Standard}

Dimethyl sulfoxide DMSO was chosen here as an internal standard for a number of reasons. i) It is miscible with most organic solvent. ii) It is easy to measure due to its physical stability as a non volatile liquid. iii) It produces a single discrete signal in the 2D-HSQC spectrum which does not overlap with other signals in the lipid profile. iv) It is chemically stable which means that it does not interact with cellular lipids under the mild conditions of extraction and NMR acquisition. In a series of calibrating ${ }^{1} \mathrm{H}_{-}{ }^{13} \mathrm{C} 2 \mathrm{D}-\mathrm{HSQC}$ spectral evaluation, NMR spectrometer was able to produce a linear response to different concentrations of DMSO between one and $25 \mathrm{mM}$ that were detected in 1:1 solution of $\mathrm{CD}_{3} \mathrm{OD}$ and $\mathrm{CDCl}_{3}$ (Figure 2.8). Similar linearity in response was obtained when three concentration of DMSO at $4 \mathrm{mM}, 8 \mathrm{mM}$ and $16 \mathrm{mM}$ were used to reference the lipid pool of $M$. smegmatis. From these concentrations, $8 \mathrm{mM}$ concentration $(0.025 \mu 1$ of DMSO per $10 \mathrm{mgs}$ of cell pellet) was chosen to provide a reference signal of reasonable integration value and without overlapping with other signals in the spectrum.

\subsubsection{Reproducibility of the Lipid Profiles}

Four cell cultures of $M$. smegmatis grown under the same growth conditions were used to investigate the reproducibility of the procedures. The integral values of three biomarker signals for PIMs, TAGs and unsaturated fatty acids (unFA) were collected as indicators for the reproducibility of the results. With the use of a standard extraction and NMR acquisition protocols and prior to any adjustment, the standard deviations for the three sets of data collected for PIMs, TAGs and unFAs were reported at 17.1\%, 17.2\% and $15.2 \%$ respectively (Figure 2.9 ). When these values were adjusted by normalization to the peak intensity of the internal standard DMSO, the values for standard deviation were improved to $11.3 \%, 14.7 \%$ and $11.4 \%$ respectively. Further improvement to $10.6 \%$, 


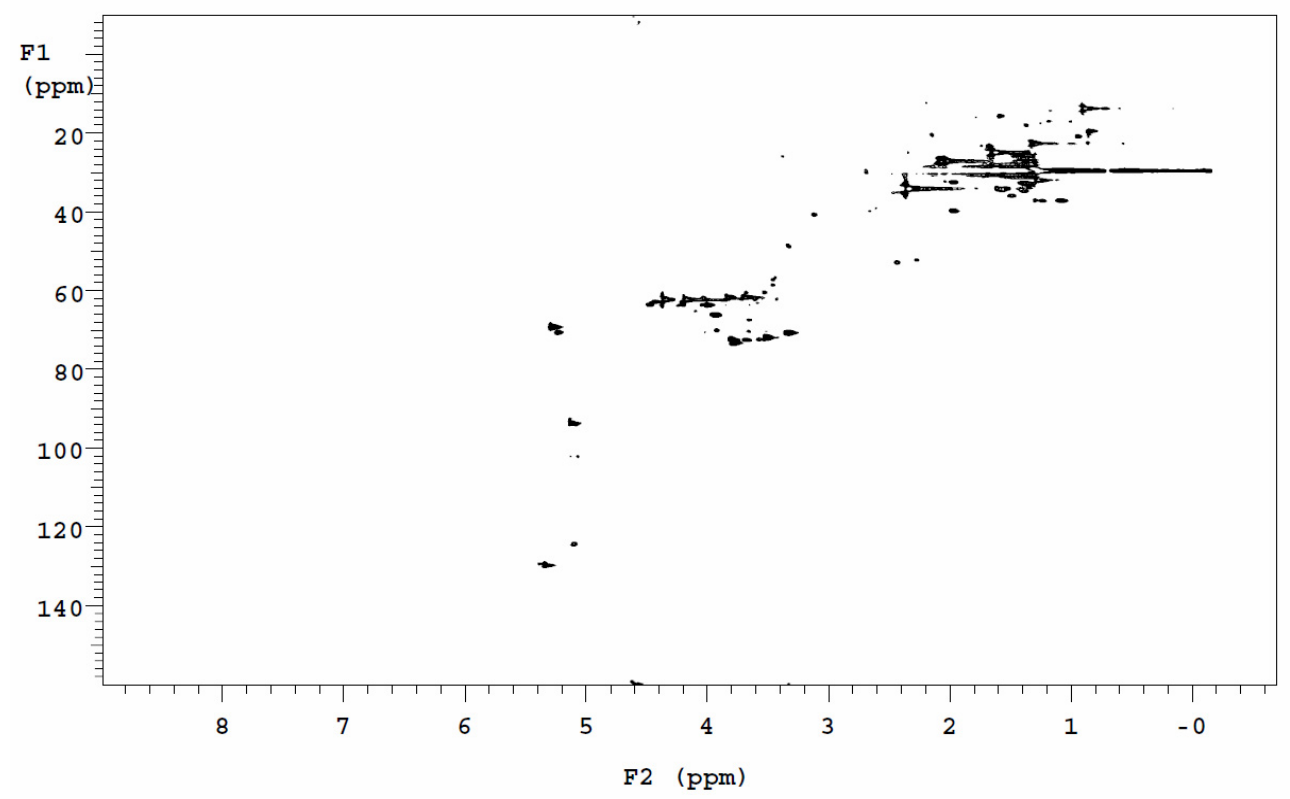

Figure 2.6 2D-HSQC Spectrum Produced by Repeated Extraction of the Cell Pellet

The cell pellet remaining after extraction of $M$. smegmatis cells by $2: 1$ mixture of $\mathrm{CDCl}_{3}: \mathrm{CD}_{3} \mathrm{OD}$ was re-extracted with the same solvent mixture in 3:1 ratio as used before. Only signals of straight chain fatty acids were observed in the lipid profile of this extract. The 2D-HSQC spectrum shown above was obtained after 10 transients and 256 increments. 

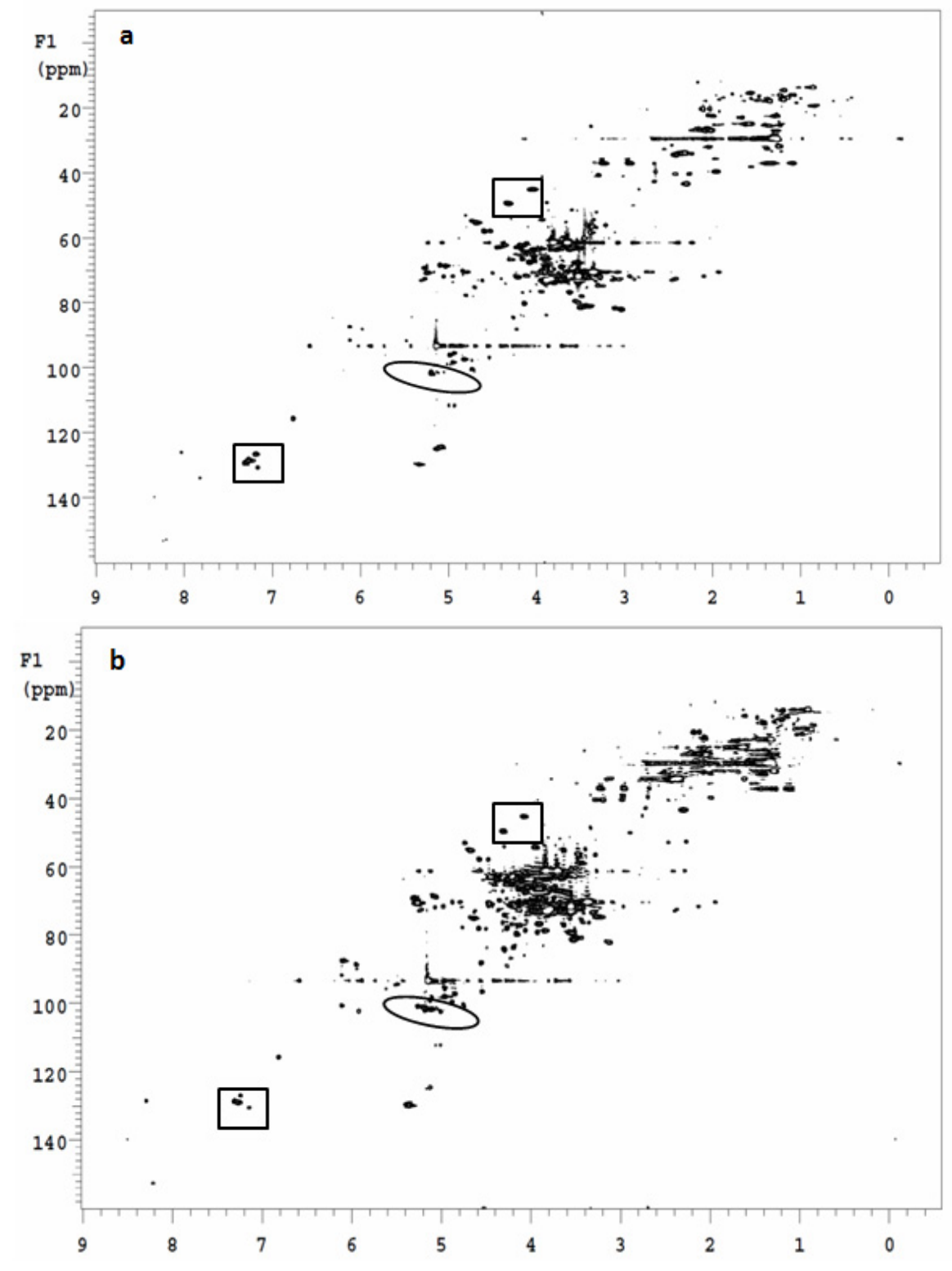

Figure 2.7 Effect of Using Acetone- $\mathrm{d}_{6}$ as the Extracting Solvent

M. smegmatis cells were extracted with either acetone- $\mathrm{d}_{6}$, (a), or a 2:1 mixture of $\mathrm{CDCl}_{3}: \mathrm{CD}_{3} \mathrm{OD}$, (b). Signals of GPL were reported at higher intensity in the acetone extract (biomarker signals of GPL are enclosed in solid squares). Also highlighted, is the loss of signals indicatives of cellular PIMs from the acetone extract (biomarker signals of PIMs are enclosed in solid ovals). 


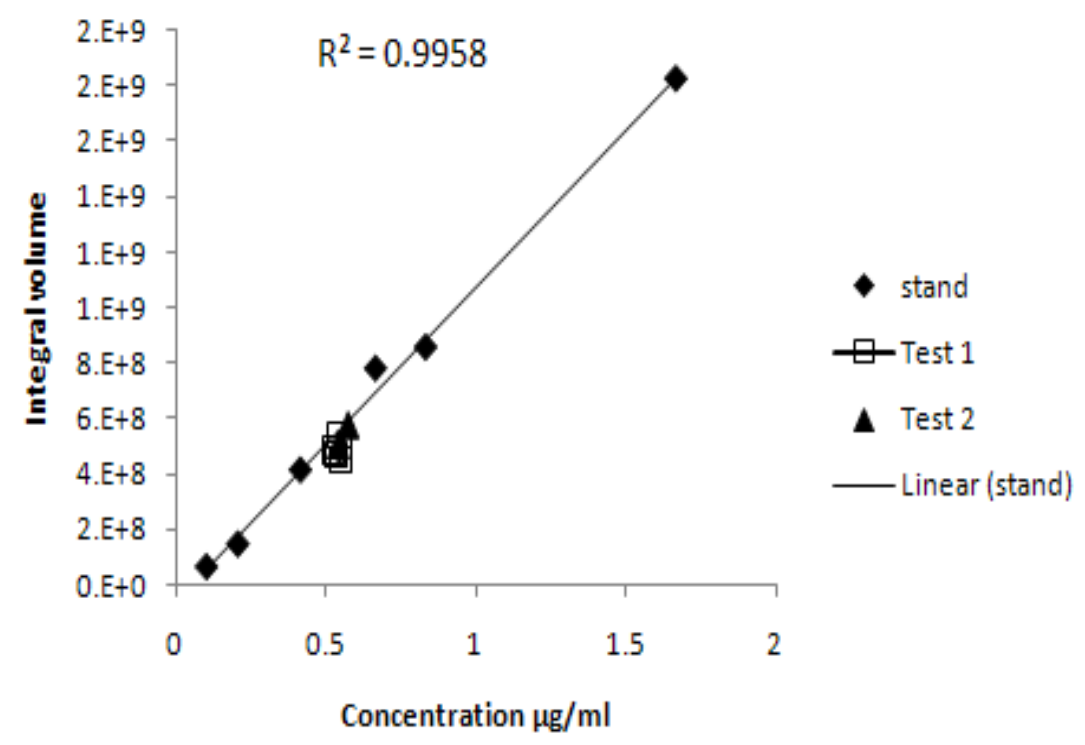

Figure 2.8 Calibrating Curve of Different Concentrations of DMSO

The integral volumes of the DMSO signals in a series of dilutions in 1:1 mixture of $\mathrm{CDCl}_{3}: \mathrm{CD}_{3} \mathrm{OD}$ are plotted as diamond $\left(\mathrm{R}^{2}\right.$ value $\left.=0.996\right)$. Also plotted on the curve are integral volumes of DMSO peak obtained through the addition of $8 \mathrm{mM}$ DMSO to the lipid extract of M. bovis BCG, (solid triangles) or M. smegmatis, (squares). 

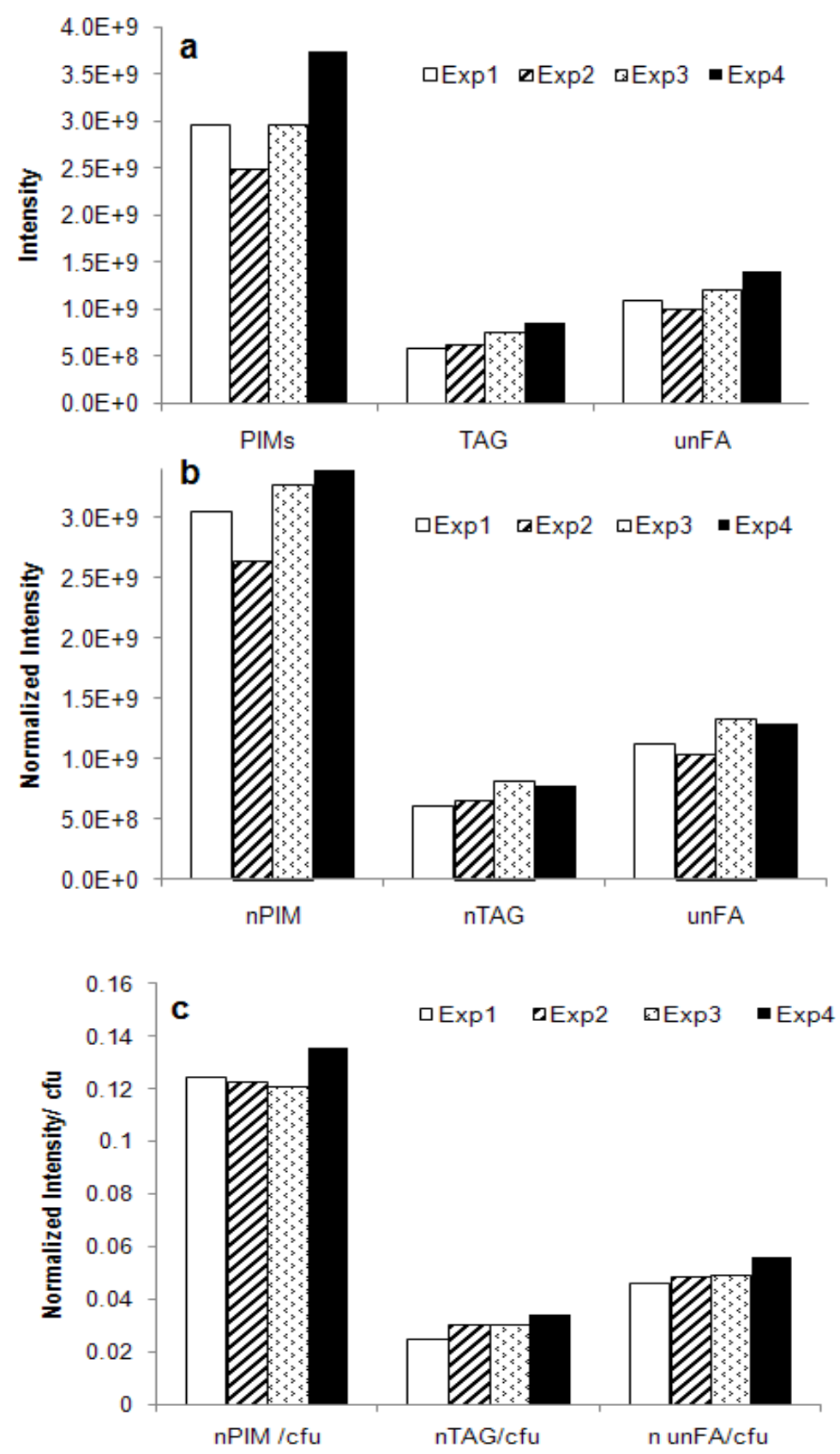

Figure 2.9 Assessment of the Reproducibility of Data Obtained from 2D-HSQC Lipid Profiles

Data are presented as integral volumes of lipid biomarker peaks, (a), or integral volumes normalized to the DMSO peak, (b), or normalized integral volumes divided by total cell number, (c). Biomarker signals used were $\delta^{1} \mathrm{H}=5.26, \delta^{13} \mathrm{C}=70.47 \mathrm{ppm}$ for PIMs, $\delta^{1} \mathrm{H}=5.30, \delta^{13} \mathrm{C}=69.31 \mathrm{ppm}$ for $\mathrm{TAG}$ and $\delta^{1} \mathrm{H}=5.12, \delta^{13} \mathrm{C}=124.62 \mathrm{ppm}$ for unFA. 
$12.9 \%$ and $8.77 \%$ was achieved when the DMSO-normalized intensities were referenced to the total number of cells used for extraction (Figure 2.9c).

\subsubsection{Lipid Fractionation through Solvent Partition}

Separation of the polar and apolar lipids can be easily achieved through a simple modification in the extraction method. When water is released from cells during extraction with $2: 1 \mathrm{CDCl}_{3}: \mathrm{CD}_{3} \mathrm{OD}$, the relative ratios of chloroform to methanol to water in the solvent mixture result in partitioning of the sample into two phases. When the two phases are separated the most lipophilic lipids are concentrated in the lower chloroformic phase while polar lipids migrate to the upper methanolic/aqueous phase. The result of this separation was the elimination of the most abundant lipids (namely TAG and acylated PIMs) in one pool leaving most glycolipids in a separate more polar pool. This resulted in the appearance of multiple signals of less abundant lipids in the HSQC spectrum of the methanolic phase, while a better resolution of some overlapping signals was also achieved.

As an example, Figure 2.10 shows the effect of solvent partition on the representation of M. bovis BCG lipid pool. Signals of mycoside B were detected exclusively in the apolar lipid spectrum while mycobactin signals were observed in the polar lipid spectrum only. Signals for the glycerol containing phospholipids that were overlapping in the total lipid pool became more abundant and better presented in the polar lipid pool when the abundant TAG and PIMs were removed into the more lipophilic phase. In other species that have different lipid compositions, cellular lipids were effectively fractioned as well. Glycopeptidolipids GPL of $M$. avium were only detected in the non polar solvent while other lipooligosaccharides remained in the polar methanolic phase (Figure 2.11). Other examples of the effect of solvent portioning are demonstrated in Figures A.11 and Figure A.12.

\subsubsection{Mycolic Acid Analysis}

Upon extraction of mycolic acids, we were able to detect all classes of mycolic acids in the 2D-HSQC mycolic acid profiles (Figure 2.12). Non overlapping biomarker signals were identified for each unique class of mycolic acids including different signals for cis and trans configuration of the cycloprapane rings and double bonds substitution. These discrete biomarker signals are summarized in Table 2.2. Among these signals, two signals can be used to represent the total mycolic acid pool. These are the $\alpha-\mathrm{CH}$ at $\delta^{1} \mathrm{H}=2.38, \delta^{13} \mathrm{C}=51.61 \mathrm{ppm}$ and the $\beta$-hydroxy $\mathrm{CH}$ at $\delta^{1} \mathrm{H}=3.65, \delta^{13} \mathrm{C}=72.15 \mathrm{ppm}$. The two signals are present in all types of mycolic acids thus they can be used as a way to estimate the total mycolic acid pool. The relative contribution of a specific type of mycolic acid can be measured by referencing the intensity of each biomarker signal to any of these two common signals. Also, an insight to the average chain length of the mycolates can be gained through measuring the intensity of the alkyl chain $\mathrm{CH}_{2}$ peak and referencing it to either the $\alpha-\mathrm{CHCO}$ or the $\beta-\mathrm{CHOH}$ signals. 

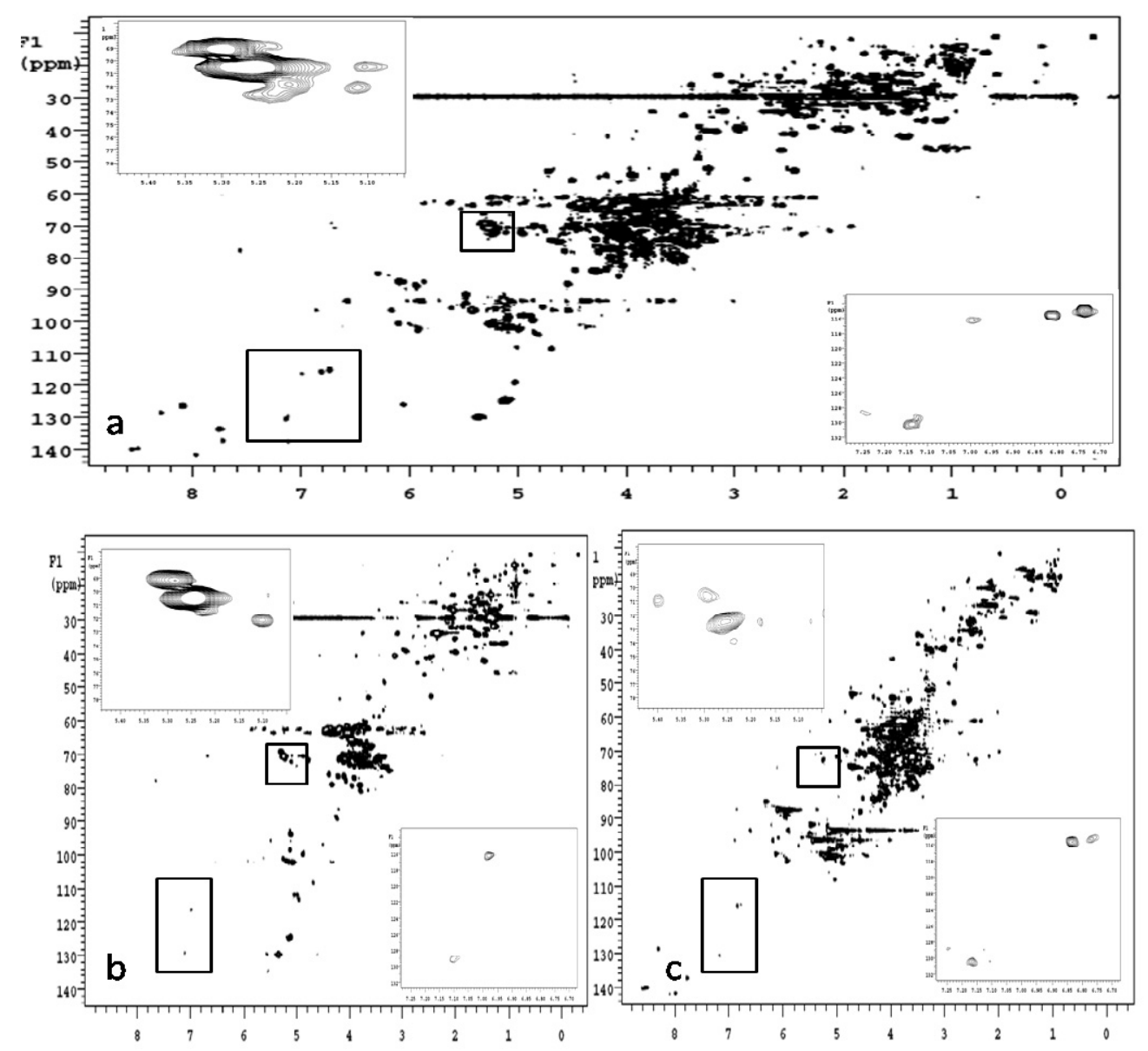

Figure 2.10 Fractionation of the Lipid Extract of $M$. bovis BCG

${ }^{1} \mathrm{H}_{-}{ }^{13} \mathrm{C}$ HSQC spectra generated from M. bovis BCG grown in ${ }^{13} \mathrm{C}$-labeled 7H9, (a), total lipid, (b), apolar lipids and (c), polar lipids. Expanded views showing detail of the biomarkers for mycobactins and mycoside B (bottom inserts, $\delta \mathrm{H}$ 6.68-7.28, $\delta \mathrm{C} 113-132.3$ ppm) and glycerol containing phospholipids (upper inserts, $\delta \mathrm{H} 5.0-5.44, \delta \mathrm{C} 67-78.8 \mathrm{ppm}$ ) have been shown in these spectra as examples of how lipids can be separated through simple fractionation. Only mycoside B is present in the apolar lipid spectrum while mycobactin signals are observed in the polar spectrum. The glycerol containing phospholipids signals are overlapping in the total lipid pool, (a). However, when the abundant TAG and PIMs are removed into the apolar solvent, less abundant species were better presented in the polar lipid spectrum. 

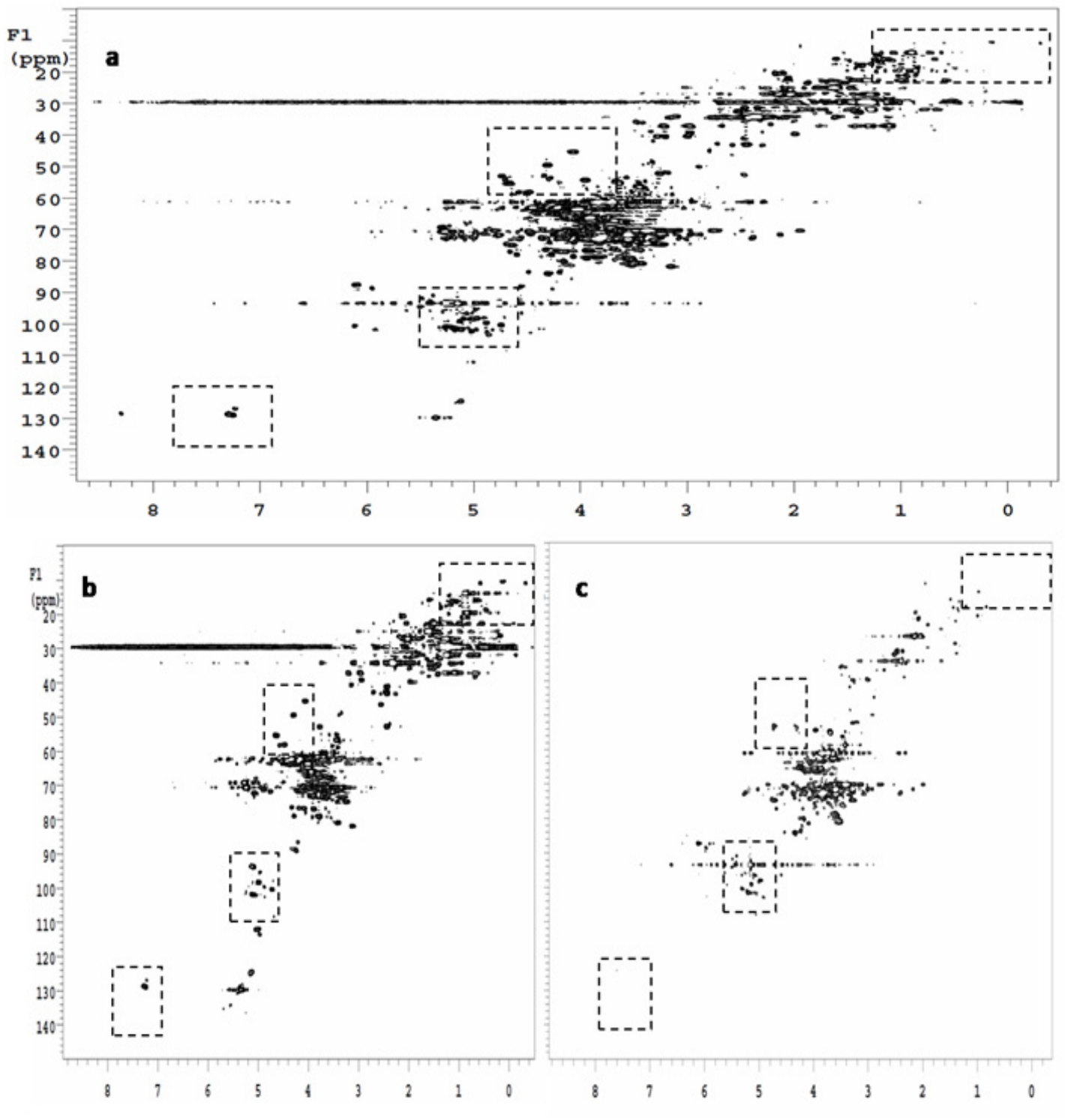

Figure 2.11 Fractionation of the Lipid Extract of M. avium

2D-HSQC profiles of the lipid extract from M. avium, total lipids, (a), nonpolar lipids, (b) and polar lipids, (c). For demonstrating lipid migration between solvent phases, areas that represent certain lipids are enclosed within dashed boxes. From the upper most part and going downward, first TDM is shown to migrate completely to the non polar solvent phase as indicated by its cyclopropyl ring signals. Second, signals for the peptide core of GPL disappeared from the polar extract due to migration to the chloroformic phase. The third area shows the partitioning of glycolipids between the non polar and polar phase as indicated from the sugar anomeric signals. Finally, signals of the phenylalanine residues of GPL are detected exclusively in the non polar solvent phase upon fractionation. 


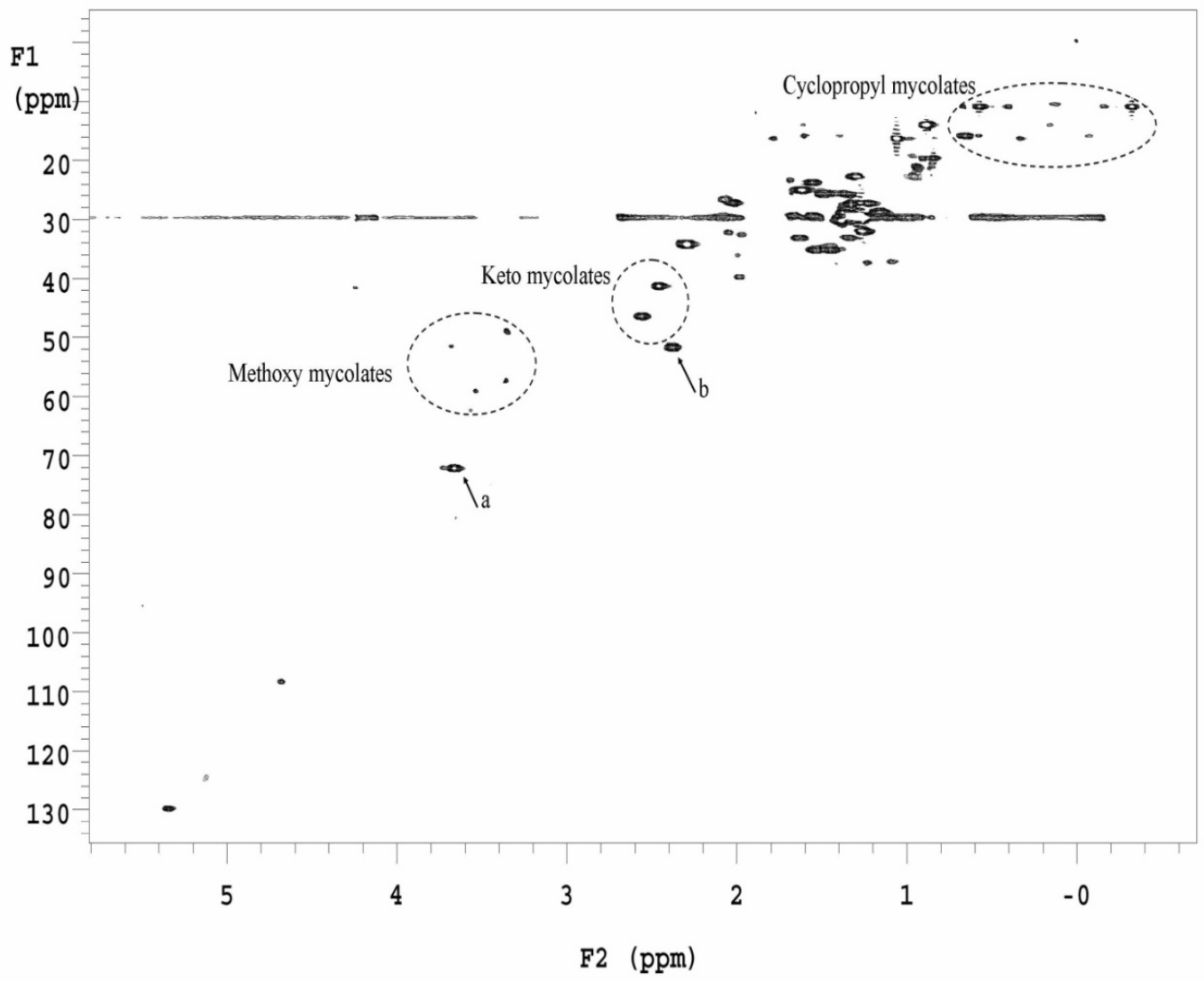

Figure 2.12 2D-HSQC Profile of Mycolic Acids Isolated from M. bovis BCG

${ }^{1} \mathrm{H}_{-}{ }^{13} \mathrm{C}$ HSQC spectrum of mycolic acids extracted from M. bovis BCG as described in text (section 2.2.3). Examples for signals common to all mycolic acid are shown by arrows (a, $\beta \mathrm{CH}-\mathrm{OH}$ and $b, \alpha \mathrm{CH}-\mathrm{COOH})$. Signals indicative to specific classes of mycolic acids are circled in dashed lines. 
Table 2.2 Chemical Shift Values of Major Mycolic Acid Biomarkers

\begin{tabular}{|c|c|c|c|c|c|}
\hline Description & $\begin{array}{c}\delta^{1} \mathbf{H} \\
\text { ppm }\end{array}$ & $\begin{array}{l}\delta^{13} \mathbf{C} \\
\text { ppm }\end{array}$ & Description & $\begin{array}{l}\delta^{1} \mathbf{H} \\
\text { ppm }\end{array}$ & $\begin{array}{l}\delta^{13} \mathrm{C} \\
\text { ppm }\end{array}$ \\
\hline Total mycolates & & & Cyclopropyl ring & & \\
\hline $\mathrm{CH}_{2} \mathrm{CO}$ & 2.38 & 51.61 & Cis $\mathrm{CH}_{2}$ ring & -0.33 & 10.89 \\
\hline $\mathrm{CH}-\mathrm{OH}$ & 3.65 & 72.15 & & 0.58 & 10.89 \\
\hline Keto Mycolate & & & Cis $\mathrm{CH}$ & 0.65 & 15.80 \\
\hline $\mathrm{CH}_{2} \mathrm{CO}$ & 2.56 & 46.49 & Trans $\mathrm{CH}_{2}$ & 0.13 & 10.43 \\
\hline Methoxy mycolate & & & Double bond & & \\
\hline $\mathrm{OCH}_{3}$ & 3.36 & 57.23 & Cis $\mathrm{CH}=\mathrm{CH}$ & 5.35 & 129.87 \\
\hline $\mathrm{CHOH}$ & 3.56 & 62.36 & Trans $\mathrm{CH}=\mathrm{CH}$ & 5.12 & 124.45 \\
\hline
\end{tabular}




\subsection{Discussion}

The chemical diversity displayed by the lipid content of the mycobacterial cells represents a great challenge for its comprehensive analysis. ${ }^{216,236}$ Previous methods developed for this process used different techniques and each has its own merits and weaknesses. The classical 2D-TLC method is favored by many researchers when the purpose is to track a specific cell wall lipid due to the simplicity of the procedures and the lack of requirement for special analytical instruments. ${ }^{136,200,237}$ When a specific molecule is being tracked, a single solvent system can be used and an optimized detection strategy (most often radiolabeling) can be employed to improve the sensitivity towards this specific molecule. ${ }^{10}$ The recent MS and LC/MS methods represent great opportunities to researchers but they ideally require significant sample manipulation to reduce ion suppression or improve HPLC resolution for reasonable data interpretation. ${ }^{215,216}$ These methods confer high sensitivity that allows the detection of minor metabolites that are hard to detect by other techniques. On the other hand, MS-based methods are inherently biased to polar lipids that can easily ionize under MS acquisition conditions.

It is crucial in the development of any global analytical technique to guarantee the representation of all components of the cellular lipid pool in their respective cellular abundance while maintaining enough flexibility to enrich any specific subpopulation if desired. In order to achieve this goal it is important to i) optimize extraction and acquisition condition for comprehensive analysis of the total lipid pool, ii) develop a standard protocol with several measures that insure the reproducibility of lipid profiling techniques, iii) investigate the possibility of enriching and targeting specific lipid subgroups and iv) provide a clear plan for maximum validation and utilization of the acquired data with an easy to apply analytical and statistical models.

\subsubsection{A Summary of a Standard Protocol for Comprehensive Analysis}

A standard protocol was developed for lipid analysis as discussed in section 2.3.1. This basic protocol uses a 2:1 mixture of $\mathrm{CDCl}_{3}: \mathrm{CD}_{3} \mathrm{OD}$ to extract pre-washed cell pellet in $3: 1$ volume per weight ratio. The lipid profiling protocol was found to be reproducible when growth conditions, the type of solvent, initial culture volumes, duration and temperature of extraction and NMR acquisitions parameters are held constant. A universal enrichment of the ${ }^{13} \mathrm{C}$-content of the cellular lipids was obtained after inoculating unlabeled cells in a growth media containing ${ }^{13} \mathrm{C}_{6}$-dextrose and ${ }^{13} \mathrm{C}_{3}$-glycerol as the main carbon source. About $20 \mathrm{mls}$ of ${ }^{13} \mathrm{C}$-enriched culture in the middle of its exponential growth was required for developing a high quality spectrum using $5 \mathrm{~mm}$ NMR probe. We were also able to use $10 \mathrm{mls}$ of culture to obtain a high quality spectrum using $3 \mathrm{~mm}$ probe. It is feasible that by the use of more sensitive NMR-sample probes such as the cryogenic probe, the initial culture volume required for a good profile can be further reduced. Such advancement in NMR acquisition and future refining of the extraction protocol can allow for the future use of NMR-based method in some in vivo settings. 


\subsubsection{Amenability of the Protocol for Both Comprehensive and Targeted Analysis}

Although the procedure discussed here focuses mainly on the analysis of the overall free cell wall lipids populations, the method can be easily modified to target a specific lipid population. For such purpose, the global ${ }^{13} \mathrm{C}$-enrichement strategy using ${ }^{13} \mathrm{C}_{6}$-dextrose and ${ }^{13} \mathrm{C}_{3}$-glycerol can be replaced by a biosynthetically-guided approach that uses specific ${ }^{13} \mathrm{C}$-labeled precursors in order to enrich signals from certain lipid population. Moreover, subpopulation of apolar lipids, acetone soluble lipids, lipooligosaccharides, covalently bound mycolic acids or others can be specifically targeted by slight modification of the extraction procedures either by switching to a different extraction solvent or by introducing additional steps prior to extraction (e.g. alkaline hydrolysis followed by extraction in case of mycolic acids).

The fractionation of the total lipid pool into smaller better resolved sub population pools can also be achieved through modification of the extraction methods. Some example methods include successive extraction of cells with deuterated solvents of increasing polarity, use of scavenger resins to pull down and fractionate anionic lipids

and cationic lipids, ${ }^{176,238}$ and the use of solvent partitions to remove very abundant signals or to separate the polar and apolar lipid pools. The latter strategy was employed here with considerable success.

\subsubsection{Biomarker Assignment through Literature Source and Isolation of Standards}

Due to the complexity of the spectra produced by this method and the incomplete spectral assignments in the literature, it is important to have a set of standards and analyze them under the same conditions as the lipid profile in order to use for effective biomarker assignment. For assigning signals in $M$. tuberculosis lipid profiles, some molecules may have low abundance in M. tuberculosis that is not sufficient to obtain enough samples to be used as a lipid standard. In these cases, structurally similar molecules (i.e. of the same lipid class) can be isolated from other species where their cellular levels are significantly higher. If necessary, 3D HCCH-TOCSY and 3D HCCHCOSY can then be used to confirm assignment of biomarker signals in the total lipid profile. This is especially useful for confirming the chemical shifts of any signals that may vary slightly between similar molecules isolated from different species. Such variations in the chemical shifts are direct results of subtle differences in structural composition or substitution. After biomarkers have been assigned in the total lipid profile, spectral sets can be compared and the relative amounts of cellular lipids can be inferred by processing the data using software such as Felix or NMR Pipe.

\subsection{Conclusion}

In conclusion, a simple method to globally analyze mycobacterial cell wall lipids was developed. While this method does not have the fine sensitivity of MS-based lipidomics approaches, it has the advantage of being suitable to analyze lipid species regardless of 
their polarity or ability to ionize and allows the simultaneous analysis of a broader range of lipids. NMR is particularly well suited for the analysis of mycobacterial lipid pool since most of these lipids have relatively high abundance as structural components of the cell wall. The lipid profiles produced by this method seem to offer a comprehensive representation of all major classes of lipids that have been reported in the literature. The rapid nature of this method, the simple procedures and the small sample size required make it a new candidate strategy for the definition of target molecules involved in virulence. The method requires only the availability of a standard high field NMR spectrometer which can be found in most academic settings. This common access can encourage a relatively wide use of this method by the mycobacteriology research community.

Potential application of this method can include several areas of mycobacteriology research that still represent serious challenges. Discovery of new genes that are involved in the cell wall biosynthesis can be achieved through such simultaneous analysis of cell wall lipids to pinpoint changes in the lipid profile as a result of gene mutation. Other application areas include studying inter and intra species variation, investigating new drugs mode of action and detecting cellular adaptation to drug treatment and other environmental stress conditions.

The methodology also has the potential to be extended to include other bacterial pathogens as long as they can be cultured in the laboratory in well defined growth medium. Most members of the actinomycetal line have chemotype IV cell walls that are rich in mycolic acids and other glycolipids. Actinomycetes include several pathogenic species as well as other species that produce unique and clinically relevant cell metabolites such as aminoglycosides produced by Streptomyces $s p .{ }^{239}$ Similar methods can be developed for targeted analysis of such metabolites by employing the right enrichment and extraction strategies. 


\section{CHAPTER 3. USE OF 2D-HSQC NMR FOR ANALYSIS OF MYCOBACTERIA CELL WALL ${ }^{2}$}

\subsection{Introduction}

\subsubsection{Mycobacteria Human Pathogens}

Mycobacteria species are mainly saprophytic but some are serious human pathogens and are implicated with devastating diseases. One of the mycobacterial diseases, TB, is caused by M. tuberculosis and claims 1.7 million lives every year while nine million new cases are reported. ${ }^{12}$ The devastation of TB is most observed in areas of sub-Saharan Africa and the densely populated parts of East Asia where the epidemic of HIV is most prevalent and less successfully controlled. ${ }^{12,29}$ The second most common mycobacterial diseas, leprosy, is caused by $M$. leprae with 250,000 reported cases. ${ }^{37}$ Both $M$. tuberculosis and $M$. leprae are intracellular pathogens that can survive and replicate inside human cells including macrophages, peripheral neurons and endothelial cells.

The third most common mycobacterial infection is that of Buruli ulcer which is endemic to some tropical areas of the world. ${ }^{241,242}$ The causative agent for Buruli ulcer, M. ulcerans, is an extracellular pathogen that mainly resides in the skin and connective tissue of the limbs. ${ }^{243,244}$ Unlike M. tuberculosis and M. leprae, in the laboratory $M$. ulcerans can only grow at temperature not higher than $32^{\circ} \mathrm{C}$ which makes it unable to cause human systemic infections. ${ }^{26,245}$ M. ulcerans infects tens of thousands of people in tropical regions with most prevalence in Ghana, Benin and Cote d'Ivoir in Africa, Malaysia and Sri Lanka in Asia and Mexico and Peru in South America and few reported cases in Japan, China and Australia. ${ }^{14}$ Another Mycobacteria pathogen M. marinum causes granulomatous cutaneous human disease in the extremities (swimmer granuloma) and systemic diseases in fish and frogs. ${ }^{43,246,247}$ This pathological spectrum is consistent with the inability of $\mathrm{M}$ marinum to grow at temperatures over $33^{\circ} \mathrm{C}$ and its common existence in environmental water.

Other human diseases can be caused by an increasing number of environmental non tuberculosis Mycobacteria (NTM). The most common infections are those by M. avium complex, or MAC, which includes M. avium subspecies avium, silvaticum and paratuberculosis and M. intracellulare. MAC infections are responsible for more than half the NTM infections and they are the most common opportunistic infections reported in HIV/AIDS patients. ${ }^{15,226,227,248}$ Other NTM include the photochromogenic M. marinum and $M$. kansasii, the scotochromogenic M. gordanae and M. scrofulacelum, and other fast growing species such as M. fortutium, M. abscessus and M. Chelonae. ${ }^{15,16,249}$ These

\footnotetext{
${ }^{2}$ Section 3.3.4 is adapted with permission. Mahrous, E.A., Lee, R.E. \& Lee, R.E. A rapid approach to lipid profiling of mycobacteria using 2D HSQC NMR maps. J Lipid Res 49, 455-63 (2008). ${ }^{240}$
} 
species can cause pulmonary TB-like disease and sometimes more severe disseminated systemic diseases especially in immunocompromised individuals. ${ }^{16,248}$

\subsubsection{Mycobacteria Animal Pathogens}

Other Mycobactyeria are serious animal pathogens with direct economic impact. Cattle can be infected with $M$. bovis which causes cow tuberculosis, a disease with similar symptoms to the human TB. Another mycobacterial disease affecting cattle is partuberculosis (also named John's disease) which is caused by M. avium subsp paratuberculosis and is characterized by severe diarrhea and granumlomateous enteritis. Other live stocks can be infected by different Mycobacteria pathogens such as $M$ scrofulaceum and M. xenopi. ${ }^{250,39}$ M. lepraemurium is another animal pathogens that infects mainly cats and rats and causes a disease with symptoms similar to human leprosy. ${ }^{250}$ On the other hand, cold blooded animals have more susceptibility to certain Mycobacteria that include M. marinum, M. liflandii, M. pseudoshittsii, M. xenopi and $M$. avium subsp intercellularae. ${ }^{5}$

\subsubsection{Old Classification of Mycobacteria}

Mycobacteria species that we recognize today live in a wide range of environments with different growth characteristics, infectivity and pathogencity. The early classification of Mycobacteria which was based on microbiological features like colony morophology and growth characteristics have resulted in long debates between researchers. Another result from the use of such classification was some literature confusion especially in the assignment of newly discovered organism as a distinct species or a variant of an existing species. Generally, the most accepted classic classification is to divide Mycobacteria into two main classes; slow growers and fast growers. ${ }^{1}$

This old classification gained credibility because most pathogenic Mycobacteria are slow growers while few of the fast growing species are associated with disease development. The same classification is still accepted now especially when the results from 16S RNA sequencing came largely in agreement with this old classification. ${ }^{11}$ Mycobacteria slow growers can be further classified based on their evolutionary relationship into multi-member clades. It is of special interest to lipidomic research that species included within the same clade were found to share a common mycolic acid pattern. ${ }^{1}$

\subsubsection{Variability in Cellular Lipid among Mycobacteria}

The variation of the cell wall composition of Mycobacteria (especially mycolic acids) has played a significant role in its classification. Distribution of mycolic acids in terms of chain length and the types of substitution have been will reported and it provided one of

the first biochemical bases for the chemotaxonomy of Mycobacteria. ${ }^{88,89,91}$ Other lipids 
that were suggested as a basis for biochemical classification of Mycobacteria due to their wide variability among different species include menaquinones, ${ }^{172,251}$ caretenoids, $^{7}$ fatty acids, ${ }^{193}$ and mycobactins. ${ }^{163,164}$ The most clinically relevant example of the utilization of such variabilties is the serotypying of MAC which depends solely on the nature of the glycosidic part attached to a largely conserved lipopeptide core of its GPL. These GPL are highly antigenic and they are often located on the bacterial surface in direct contact with the host environment. Based on their oligosaccharide, these GPL can elicit different immunological reactions that serve as the basis for MAC serotypying. ${ }^{96,155}$

\subsubsection{Value of Lipid Profiling of Different Mycobacteria Species}

With the routine use of 16S RNA sequencing as a rapid and accurate method for taxonomical classification, Mycobacteria lipid profiling is no longer used for this purpose. However, it can be used to provide valuable information about the participation of certain lipids in several cellular processes that include virulence, niche adaptation and stringent response. Some evolutionary relationships between species can also be inferred from such profiles because cell lipids can be considered as ultimate gene products. Some lipid profiling techniques such as HPLC are still used by CDC as a fast tool for diagnosis of TB cases to characterize the causative agent for TB infections to the species level. ${ }^{187}$ This chapter discusses the variability in the cell wall metabolites among different Mycobacteria and how it corresponds to variations in their growth characteristics, pathogenicity and evolutionary relationship. It also further validates the use of NMR as a rapid tool for monitoring the cell wall metabolites of Mycobacteria as well as other microorganisms. The technique can also provide more comprehensive insights into the cell wall composition when combined with the previously developed HR-MAS method for monitoring cell wall polysaccharides ${ }^{189}$ (see section 1.7.2.2). This combination is very reasonable as both methods require ${ }^{13} \mathrm{C}$-enrichement of the cellular metabolites and both go through similar steps of sample preparation and data processing.

\subsection{Materials and Methods}

\subsubsection{Strains and Growth Conditions}

Mycobacterial cells were grown in Middlebrook $7 \mathrm{H} 9$ supplemented with $0.2 \%$ of ${ }^{13} \mathrm{C}_{6}$-dextrose, $0.2 \%{ }^{13} \mathrm{C}_{3}$-glycerol, $0.05 \%$ tween and $5 \% \mathrm{BSA}$ at $36.5^{\circ} \mathrm{C}$ with continuous shaking at $250 \mathrm{rpm}$. Fast growing species (M. smegmatis ATCC $\mathrm{mc}^{2} 155$, M. fortuitum ATCC 6841, M. phlei, M. abscessus ATCC 19977) were inoculated at initial optical density $\mathrm{O}_{2} \mathrm{D}_{\lambda 600}=0.005$, while slow growing species (M. bovis BCG, M. tuberculosis, $M$. avium ATCC25291) were inoculated at initial $\mathrm{OD}_{\lambda 600}=0.05$. M. ulcerans $1615, M$. liflandii XL-5 and M. marinum 128 were grown at $31^{\circ} \mathrm{C}$ without shaking in tissue culture flask (see Table 3.1 for a list of the species used). Corynebacterium glutamicum, Bacillus subtilis and B. anthracis Sterne (a vaccine strain) were grown in Brain Heart Infusion media (BHI) made from $1.75 \% \mathrm{BHI}, 0.2 \%{ }^{13} \mathrm{C}_{6}$-dextrose, $1 \%$ tryptone, 
Table 3.1 Summary of the Mycobacteria Species Investigated in This Study

\begin{tabular}{lcll}
\hline Species & $\begin{array}{c}\text { Growth } \\
\text { Temperature }{ }^{\circ} \mathbf{C}\end{array}$ & Pathogenicity & Main Host \\
\hline Slow growers & 36.5 & Opportunistic & Human \\
M. avium & 36.5 & Vaccine strain & Human \\
M. bovis BCG & 36.5 & Pulmonary, Systemic & Human \\
M. tuberculosis & 31 & Systemic & Frog \\
M. liflandii & 31 & Systemic & Fish \\
M. marinum & 31 & Skin, Connective & Human \\
M. ulcerans & & tissue & \\
Fast growers & 36.5 & Opportunistic & Human \\
M. abscessus & 36.5 & Opportunistic & Human \\
M. fortitum & 36.5 & None & None \\
M. phlei & 36.5 & None & None \\
M. smegmatis & & &
\end{tabular}


$0.5 \% \mathrm{NaCl}$ and $0.25 \%$ disodium phosphate. Cells were incubated with continuous shaking at $250 \mathrm{rpm}$ at $36.5^{\circ} \mathrm{C}$.

\subsubsection{Sample Preparation for HR-MAS}

Cells from 40-50mls culture were harvested by centrifugation at $3700 \mathrm{xg}$ for 10 minutes then washed twice with $5 \mathrm{mls}$ of $\mathrm{D}_{2} \mathrm{O}$ with removal of the aqueous supernatant by centrifugation in between. The washed cell pellet was then transferred to a pre-weighed micro-centrifuge tube using $1 \mathrm{ml}$ of $\mathrm{D}_{2} \mathrm{O}$. After centrifugation at $21,000 \mathrm{xg}$ for 10 minutes, cells were re-suspended in minimal amount of $\mathrm{D}_{2} \mathrm{O}(\sim 200 \mu \mathrm{l})$. About $40 \mu \mathrm{l}$ of the cell suspension was then transferred to an HR-MAS rotor for NMR acquisition.

\subsubsection{Lipid Extraction}

Total cell lipids were extracted from the previously washed cell pellets after the removal of all remaining $\mathrm{D}_{2} \mathrm{O}$. A $2: 1$ mixture of $\mathrm{CDCl}_{3}: \mathrm{CD}_{3} \mathrm{OD}$ was added to the cell pellet on basis of the wet pellet weight ( $3 \mu \mathrm{l}$ per $1 \mathrm{mg}$ of cells) and cells were extracted for 90 minutes at $37^{\circ} \mathrm{C}$ with continuous shaking at $250 \mathrm{rpm}$. After extraction, more $\mathrm{CD}_{3} \mathrm{OD}$ ( $1 \mu \mathrm{l}$ per $1 \mathrm{mg}$ of original cell weight) was added to the extraction mixture to obtain a single phase solution which was then separated from the cell debris by centrifugation for 10 minutes at $21,000 \mathrm{xg}$ at room temperature. The lipid extract was then transferred to a $5 \mathrm{~mm}$ NMR tube for NMR data acquisition.

\subsubsection{Sterility Testing}

For safety regulation, the organic extract of $M$. tuberculosis $\mathrm{H}_{37} \mathrm{Rv}$ was tested for sterility. The lipid extract was air dried in a biosafety level-3 cabinet then re-suspended in one milliliter of sterile Middlebrook $7 \mathrm{H} 9.100 \mu \mathrm{l}$ of the cell suspension was inoculated to Middlebrook $7 \mathrm{H} 11$ agar plates. No growth was observed after incubation at $37^{\circ} \mathrm{C}$ for six weeks.

\subsubsection{Isolation of PGL from M. liflandii}

M. liflandii cells were grown to stationary phase in Middlebrook 7H9, harvested by centrifugation and subsequently extracted with ethanol. The ethanolic extract was dried in vacuo. The dried residue (80mg) was re-suspended in $\mathrm{CHCl}_{3}$ and applied to a Biotage SP1 flash chromatography system equipped with a Biotage Si 12+M silica gel column. The column was eluted using a $\mathrm{CHCl}_{3}$-to- $\mathrm{CHCl}_{3} / \mathrm{CH}_{3} \mathrm{OD} 9: 1(\mathrm{v} / \mathrm{v})$ linear gradient. The major PGL was detected through TLC analysis using $\mathrm{CHCl}_{3} / \mathrm{CH}_{3} \mathrm{OH}(95: 5, \mathrm{v} / \mathrm{v})$ as the developing system $\left(\mathrm{R}_{\mathrm{f}}\right.$ of $\left.\mathrm{PGL}=0.7\right)$. Lipid spots were visualized using cerric ammonium sulfate in $2 \mathrm{M}$ sulfuric acid after charring. Fractions containing pure PGL were pooled and dried in vacuo. 


\subsubsection{NMR Acquisition}

For solid state NMR, the MAS rotor containing $40 \mu 1$ of cell suspension was inserted at the magic angle in $5 \mathrm{~mm}$ GHx nanoprobe. The 2D-HSQC sequences were recorded using Varian Inova $500 \mathrm{MHz}$ spectrometer at $24^{\circ} \mathrm{C}$ degrees with continuous spinning at $2000 \mathrm{~Hz}$. The 2D-HSQC spectra were acquired for 8 transients and 256 increments. For liquid profiling, the 2D-HSQC experiments were recorded using a triple resonance trpfg probe for 10 transient and 256 increments. NMR data were processed using either VNMR or Felix-NMR software.

\subsection{Results and Discussion}

\subsubsection{Characteristic Features of Some Species}

The use of NMR methods, both solid state and liquid NMR, can provide a rapid tool to gain insights to the structural features of the cell wall polysaccharides and lipid components respectively. Generally, No significant difference was observed in the AG signals between the ten species tested (See Appendix B, Figures B.1-B.10). This observation is consistent with the finding of Daffe at al who concluded through degradative analysis and the use of GC/MS that AG structure is well conserved among Mycobacteria. ${ }^{81}$ Some quantitative and qualitative differences were reported in LAM signals. The relative abundance of LAM signals was significantly higher in some species such as $M$. bovis and M. ulcerans as opposed to other species like M. smegmatis ${ }^{252}$ (Figure 3.1a-e). Other reported intra-species variabilities were related to the type of capping of the terminal arabinan chains in LAM which follows one of three patterns; mannose capping in manLAM, phospho inositol capping in PiLAM and no terminating caps as in araLAM. Characteristic signals of manLAM can be detected at $\delta^{1} \mathrm{H}=3.59 / 3.69$, $\delta^{13} \mathrm{C}=68.13 \mathrm{ppm}$ as in the case of $M$. bovis BCG. ${ }^{189}$ While those of PiLAM are detected at $\delta^{1} \mathrm{H}=3.88 / 4.12, \delta^{13} \mathrm{C}=66.20 \mathrm{ppm}$ as in the case of M. smegmatis ${ }^{253}$ (Figure 3.1f-j).

In liquid NMR, signature lipids of different species can be readily detected in their corresponding lipid profiles. Among the ten species investigated here, three can be easily separated out (M. avium, M. smegmatis and M. abscessus) by the dominance of GPL signals in their lipid profiles at $\delta^{1} \mathrm{H}=7.27, \delta^{13} \mathrm{C}=128.71$ and $\delta^{1} \mathrm{H}=4.31, \delta^{13} \mathrm{C}=49.45 \mathrm{ppm}$ (Figure B.11, Figure B.12 and Figure B.13). M. avium is the only slow growing species in this group and it produces a significant amount of cyclopropyl mycolates as indicated by the two signals at $\delta^{1} \mathrm{H}=-0.33 / 0.58, \delta^{13} \mathrm{C}=10.73 \mathrm{ppm}$ (Figure B.11). M. smegmatis, on the other hand, lacks those signals as well as signals of the methyl branched fatty acids that can be easily detected in M. abscessus (Figure B.12 and B.13).

Among other Mycobacteria used in this study, three species grow at significantly lower temperature namely $M$. marinum, M. liflandii and $M$. ulcerans. The presence of phenol phthiocerol in very high abundance is a major characteristic of these three species as indicated by its characteristic aromatic signals at $\delta^{1} \mathrm{H}=6.98, \delta^{13} \mathrm{C}=129.47$ and $\delta^{1} \mathrm{H}=6.7$, 

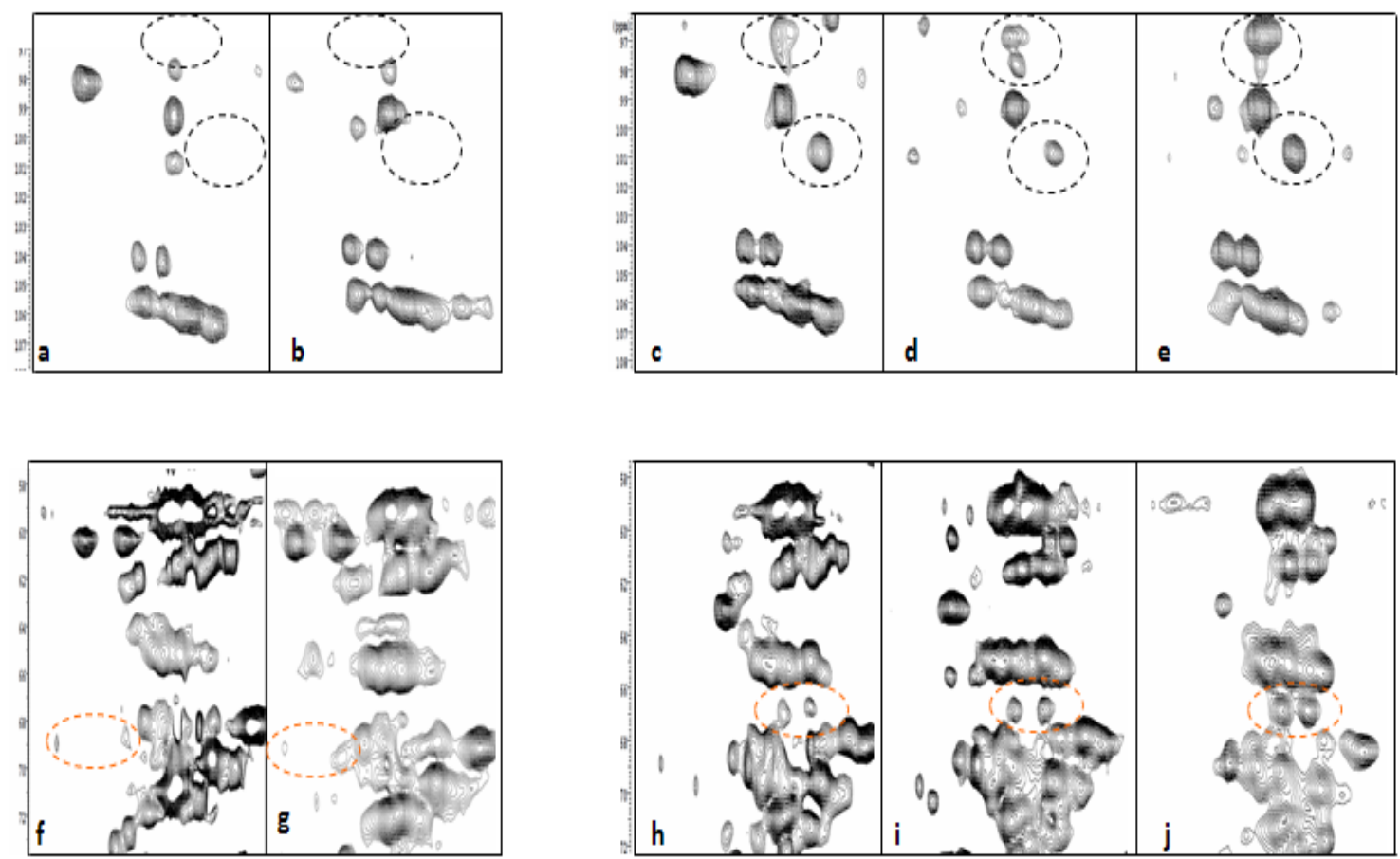

Figure 3.1 Interspecies Variabilities in the Cell Wall LAM as Observed in the HR-MAS Spectra of Different Mycobacteria

Partial windows of the HR-MAS 2D-HSQC spectra of different Mycobacteria. Upper panel: LAM signals detected at $\left(\delta^{1} \mathrm{H}=4.81, \delta^{13} \mathrm{C}=99.70\right),\left(\delta^{1} \mathrm{H}=5.02, \delta^{13} \mathrm{C}=98.08\right)$ and $\left(\delta^{1} \mathrm{H}=5.05, \delta^{13} \mathrm{C}=98.13 \mathrm{ppm}\right)$ and circled in black dashed lines are almost absent from the fast growing species $M$. abscessus, (a), and M. smegmatis, (b). The same signals are detected with high intensity in $M$. bovis BCG, (c), M. tuberculosis $\mathrm{H}_{37} \mathrm{Ra}$, (d), and $M$. ulcerans, (e).

Lower panel: Variation in the type of substitution in LAM as detected by HR-MAS cell profiles. Signals indicative of phosphoinositol capping were detected in both $M$. abscessus, (f), and M. smegmatis, (g), at $\delta^{1} \mathrm{H}=3.88 / 4.12, \delta^{13} \mathrm{C}=66.20 \mathrm{ppm}$ as circled in red. Signals indicative of mannose capping were detected at $\delta^{1} \mathrm{H}=3.59 / 3.69, \delta^{13} \mathrm{C}=68.13$ ppm in M. bovis BCG, (h), M. tuberculosis $\mathrm{H}_{37} \mathrm{Ra}$, (i), and M. ulcerans, (j), as circled in red. 
$\delta^{13} \mathrm{C}=115.01$ (Figure B.14). M. ulcerans can be further characterized by the low abundance of most major glycolipids and triacyl glycerols (Figure B.15). In fact, signals for TAG were not detected in the lipid profile of M. ulcerans using this method. Meanwhile, the lipid profiles of M. liflandii and M. marinum displayed some degree of similarity. However, M. marinum can be distinguished by a more complex profile which is significantly rich in glycolipids and carotenoids (Figure B.16 and Figure B.17).

M. bovis $\mathrm{BCG}$ and M. tuberculosis $\mathrm{H}_{37} \mathrm{Rv}$ have very similar lipid profiles but $M$. bovis $\mathrm{BCG}$ can be distinguished by the presence of the two aromatic signals of its phenolic glycolipid (mycoside $\mathrm{B}$ ) at $\delta^{1} \mathrm{H}=7.13, \delta^{13} \mathrm{C}=129.39$ and $\delta^{1} \mathrm{H}=7.00, \delta^{13} \mathrm{C}=116.52$ and the relatively low abundance of methyl branched fatty acids and PDIM as inferred from the low intensity of methyl groups signals at $\delta^{1} \mathrm{H}=1.16, \delta^{13} \mathrm{C}=19.69, \delta^{1} \mathrm{H}=1.05, \delta^{13} \mathrm{C}=17.37$ and $\delta^{1} \mathrm{H}=0.99, \delta^{13} \mathrm{C}=18.11$ (Figure B.18). On the contrary, M. tuberculosis produces significantly higher amounts of PDIM and lower amounts of acylated glycerols (Figure B.19).

The saprophytic M. phlei is easily characterized by the production of large amount of trehalose phleiates ${ }^{63}$ compounds that are easily detected through the presence of multiple olefinic signals in the range between 5.00 and $5.60 \mathrm{ppm}$ (Figure B.20). M. fortitum, a fast grower, produces a lipid profiles that displays a lack of all signals of cyclopropyl mycolates, GPLs and PGLs. It is characterized, however, by the presence of a form of acylted trehalose previously designated mycoside $\mathrm{F} .{ }^{118}$ This lipid molecule is represented in the lipid profile by a cluster of methyl groups at $\delta^{1} \mathrm{H}=0.7-1.0 \mathrm{ppm}$ and $\delta^{13} \mathrm{C}=15-22$ ppm (Figure B.21).

\subsubsection{Comparison between Slow Growers and Fast Growers}

Fast growing species included in this discussion, M. smegmatis, M. phlei, M. fortuitum and $M$. abscessus, have an average doubling time of about 2-4 hours. The slow growing Mycobacteria investigated here include M. bovis, M. tuberculosis and M. avium and have doubling time of 12 hours or longer. We limited our comparison to these two groups because both can grow in the same media and under the same growth conditions. As expected, the HR-MAS profiles showed similar AG structural characteristics in both groups but variations were observed in LAM and lipids signals. LAM signals at ${ }^{1} \mathrm{H}=4.81$, $\delta^{13} \mathrm{C}=99.70, \delta^{1} \mathrm{H}=5.02, \delta^{13} \mathrm{C}=98.08$ and $\delta^{1} \mathrm{H}=5.05, \delta^{13} \mathrm{C}=98.13 \mathrm{ppm}^{189}$ were detected at higher intensity in M. tuberculosis $\mathrm{H}_{37} \mathrm{Ra}$ and $M$. bovis $\mathrm{BCG}$ and at lower intensity in $M$. abscessus , M. fortitum, M. phlei and M. smegmatis (Figures 3.2a, B.1, B.4, B.7, B.8).

Also in all HR-MAS spectra of the fast growing species, signals for TAG at $\delta^{1} \mathrm{H}=5.13$, $\delta^{13} \mathrm{C}=69.00, \delta^{1} \mathrm{H}=4.19 / 3.99, \delta^{13} \mathrm{C}=62.01 \mathrm{ppm}$ were easily detected which were absent from the spectra of slow growing species (Figure 3.2b). This observation was further confirmed in the corresponding lipid profiles as fast growing species clearly produced larger amounts of TAG and fatty acids as indicated from the integral values of TAG peaks in their lipid profiles (Figure 3.2c). On the contrary, polyketides-based lipids such as PGL and PDIM were detected at higher intensity in the slow growers (Figure 3.2d). 

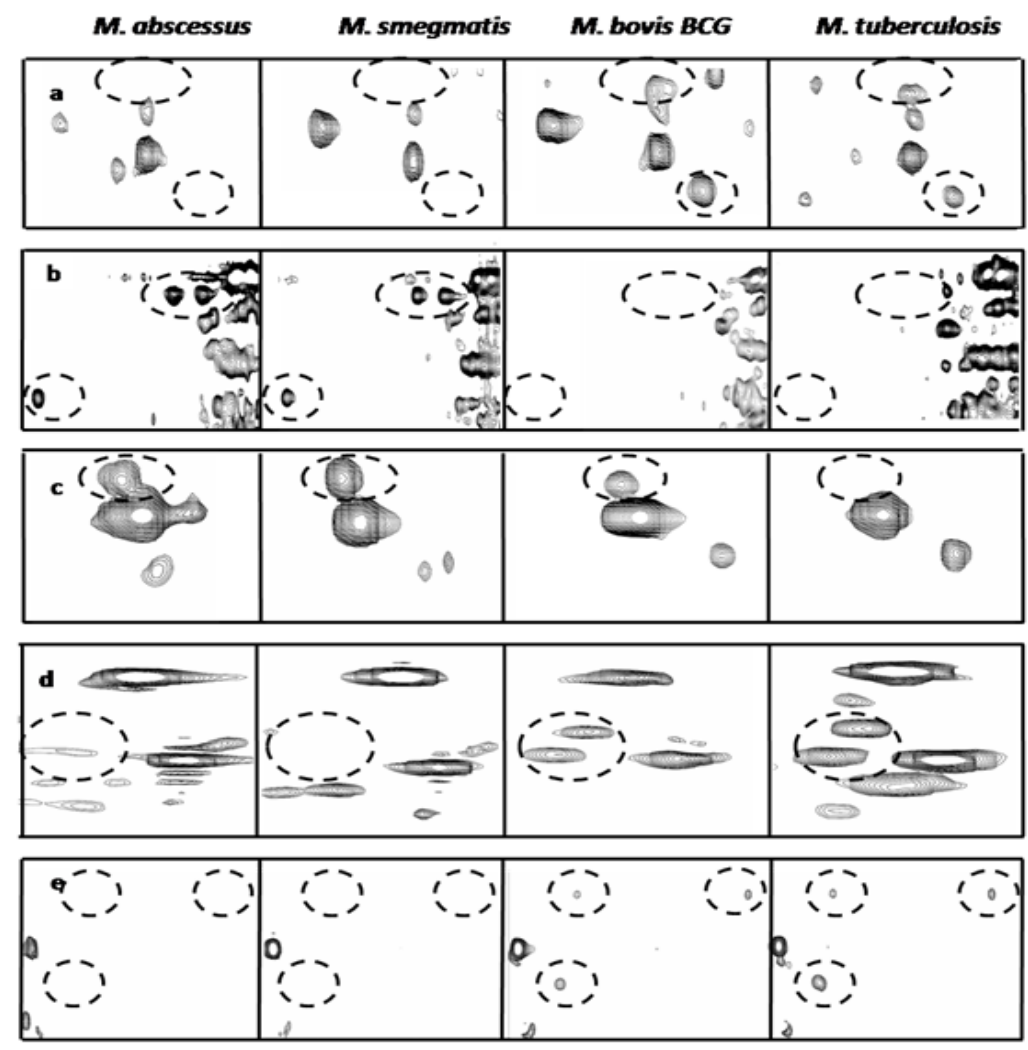

Figure 3.2 Comparison between the HR-MAS and Lipid Profiles of Some Fast Growing and Slow Growing Mycobacteria

Panel (a), differences were observed in the intensity of LAM signals' most notably in the intensity of the terminal $\alpha$-DMan $p$ signal at $\delta^{1} \mathrm{H}=4.81, \delta^{13} \mathrm{C}=99.70,2-\alpha$-D-Man $p$ at $\delta^{1} \mathrm{H}=5.02, \delta^{13} \mathrm{C}=98.08$ and 2,6- $\alpha$-D-Man $p$ at $\delta^{1} \mathrm{H}=5.05, \delta^{13} \mathrm{C}=98.13 \mathrm{ppm}$ in the HR-MAS whole cell profile of $M$. abscessus, M. smegamatis, M. bovis BCG and M. tuberculosos. Panels (b) and (c), variabilities in the amount of TAG produced by fast growers and slow growers as indicated in both the HR-MAS profile, (b), and the total lipid profile, (c).

Signals of $\mathrm{CH}$ of glycerol at $\delta^{1} \mathrm{H}=5.13, \delta^{13} \mathrm{C}=69.00 \mathrm{ppm}$, and $\mathrm{CH}_{2}$ at signals $\delta^{1} \mathrm{H}=4.19$ / $3.99, \delta^{13} \mathrm{C}=62.01 \mathrm{ppm}$ are detected in HR-MAS profiles of only $M$. abscessus and $M$. smegmatis. Similar results were observed in the lipid profiles where TAG signals were observed with higher intensity in the fast growing species. The lipid profile of $M$. bovis BCG show moderate levels of TAG that could not be detected in the HR-MAS probably due to short T2 relaxation. Panel (d), signals of the methyl groups indicative of methyl branched lipids such as phthienoic and mycocerosic acids were detected at high intensity in the lipid profiles of slow growers at $\delta^{1} \mathrm{H}=1.05, \delta^{13} \mathrm{C}=17.37$ and $\delta^{1} \mathrm{H}=1.16, \delta^{13} \mathrm{C}=19.69$ ppm. Signals of such methyl groups can be seen at low levels in M. abscessus while they were completely absent from the lipid profile of $M$. smegmatis. Panel (e), signals of cyclopropyl mycolates were detected exclusively in the lipid profiles of slow growers at $\delta^{1} \mathrm{H}=-0.33 / 0.58, \delta^{13} \mathrm{C}=10.73$ and $\delta^{1} \mathrm{H}=0.65, \delta^{13} \mathrm{C}=15.80 \mathrm{ppm}$. 
Although both groups displayed signals indicative of trehalose dimycolates at ${ }^{1} \mathrm{H}=2.47$, $\delta^{13} \mathrm{C}=52.81$ and $\delta^{1} \mathrm{H}=4.44 / 4.24, \delta^{13} \mathrm{C}=98.08 \mathrm{ppm}$, signals of cyclopropyl mycolates were absent from the lipid profiles of most fast growers (Figure 3.2e). However, these signals were observed with low abundance in M. phlei.

It is worth mentioning that the line that divides Mycobacteria into slow and fast growers overlaps to great extent with the line that divides them into pathogens and non pathogens. Although some of these previous observations are best looked at in the light of variable growth rates, others may be better understood in the context of virulence and pathogenicity. For example, the disparity in the levels of the structurally simple TAG and the complex polyketides between the two groups can be explained by differences in the metabolic rates of the bacteria and the biosynthetic energy burden associated with these lipids. Other observations such as the abundance of cyclopropyl mycolates and the presence of mannose capped LAM seem to be in better agreement with the pathogenicity and virulence argument. As a supporting evidence, the two totally non pathogenic species investigated in this study (M. smegmatis and M. phlei ) both are fast growers and both are devoid of most of immunogenic cell wall components previously identified in Mycobacteria e.g. manLAM, PDIM and PGL. Other pathogenic Mycobacteria that were not included in this comparison like M. ulcerans and M. marinum produce one or more of these molecules and will be discussed in the following section.

\subsubsection{Comparison between Intracellular and Extracellular Pathogens}

M. ulcerans is one of the few Mycobacteria that can be detected in the extracellular space in the infected host tissue (in this case human skin and connective tissues) ${ }^{243,244}$ In order to limit the participation of multiple factors in this comparison, M. ulcerans was compared only to M. marinum an intracellular human and fish pathogen ${ }^{25,26}$ that was grown under the same laboratory conditions for the purpose of this study. Moreover, $M$. marinum is closely related to $M$. ulcerans taxonomically and is thought to be the evolutionary ancestor of M. ulcerans. Based on their HR-MAS spectra, M. ulcerans and M. marinum share a similar AG structure but some differences were observed in LAM signals. Most of the LAM signals were detected at higher intensity in M. ulcerans as opposed to most Mycobacteria. These high levels can be observed in the signals of 6- $\alpha$ Man $p$ at ${ }^{1} \mathrm{H}=4.81, \delta^{13} \mathrm{C}=99.70$ and that of the terminal $\mathrm{D}-\alpha-\operatorname{Man} p$ at $\delta^{1} \mathrm{H}=4.93$, $\delta^{13} \mathrm{C}=102.19$ ppm while AG signals in both species were recorded at comparable intensity (Figure 3.3a,b). Also evidence from the HR-MAS suggests more frequent mannose capping of LAM in the case of M. ulcerans as indicated by both the abundance of the $\mathrm{t}-\alpha-\mathrm{Man} p$ as mentioned above and the high intensity of the mannose capped arabinan $\mathrm{CH}_{2}$ signals recorded at $\delta^{1} \mathrm{H}=3.59 / 3.69, \delta^{13} \mathrm{C}=68.13 \mathrm{ppm}$ (Figure 3.3a,b).

More stark differences can be observed between the two species by examining their lipid profiles. While M. marinum exhibits a lipid profile with some similarities to that of other pathogenic species like M. bovis and M. tuberculosis that display a wide variety of glycolipids and lipooligosaccharides, the lipid profile of $M$. ulcerans contains only signals of PIMs and trehalose dimycolate in the carbohydrate region (Figure 3.3c,d). 
Figure 3.3 Comparison between the Extracellular M. ulcerans and the Intracellular $M$ marinum

HR-MAS profiles of M. ulcerans, (a), and M. marinum, (b). The two profiles indicate the presence of manLAM in both species (signals at $\delta^{1} \mathrm{H}=3.59 / 3.69, \delta^{13} \mathrm{C}=68.13 \mathrm{ppm}$ are circled in red in the upper insert). However, substantial difference can be observed between the levels of manLAM detected in each case. Higher levels were detected in $M$. ulcerans as shown by the intensity of the LAM signals in the lower inserts. Also in the same insert the anomeric signal of trehalose base molecules can be detected at higher levels in the HR-MAS spectrum M. marinum (the anomeric signal of trehalose is circled in blue in both spectra).

The lipid profile of M. ulcerans, (c), is strikingly different from that of M. marinum, (d), in the relative amounts of PDIM and glycolipids produced. M. ulcerans lipid pool shows limited variety of glycolipids signals when compared to that of M. marinum (the glycolipid region is circled in the two lipid profiles). The aromatic signals for phenol phthiocerol and its glycosylated derivative (mycoside $\mathrm{G}$ ) are highlighted in a rectangular box in the lipid profile of M. ulcerans and M. marinum respectively. These signals are shifted downfield in the lipid profile of M. marinum due to glycosylation. Also in the two spectra, the location of TAG biomarker signals is indicated by a black arrow. This signal was not detected in the lipid profile of M. ulcerans. 

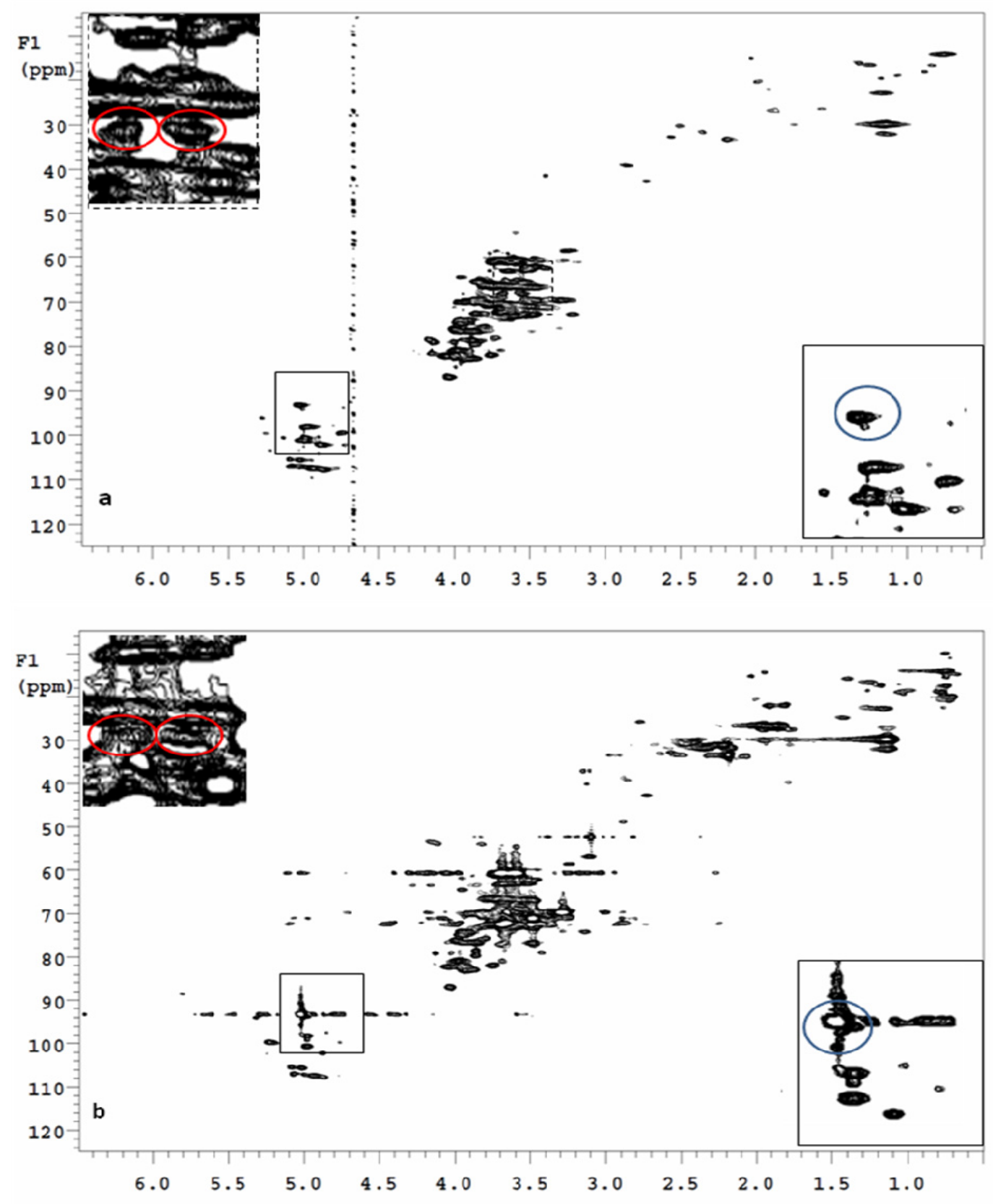

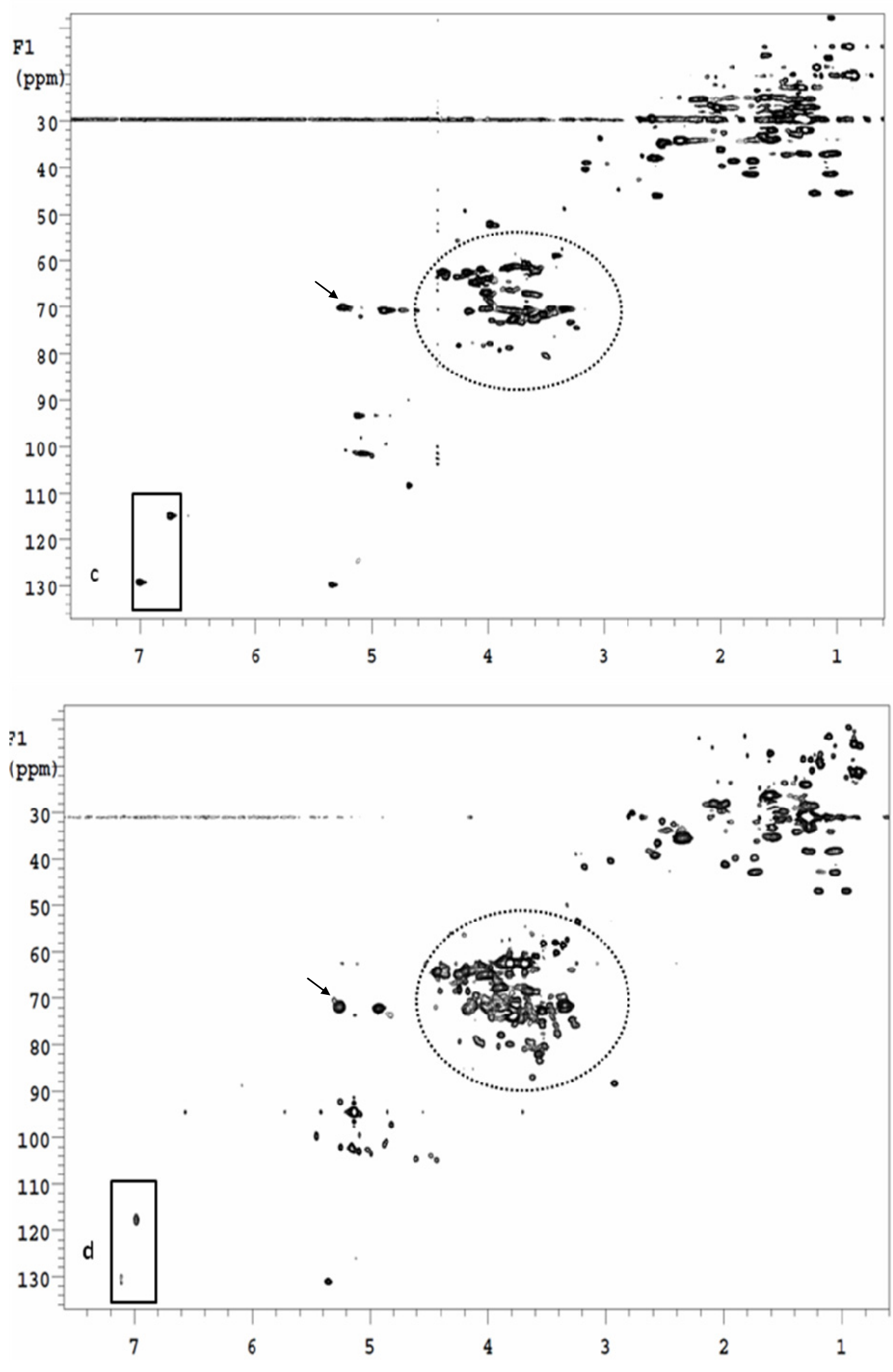

Figure 3.3 (Continued) 
Under the growth conditions used, M. ulcerans produced unusually high amounts of lipophilic waxes that include PDIM, phthiocerol, phthidiolone and their aromatic derivatives. Meanwhile, signals for TAG were not detected in M. ulcerans based on the detection limit of this method. In addition, signals characteristic of M. ulcerans secreted toxin (mycolactone) were recorded only after long acquisition time at $\delta^{1} \mathrm{H}=5.04$, $\delta^{13} \mathrm{C}=131.21$ and $\delta^{1} \mathrm{H}=4.90, \delta^{13} \mathrm{C}=76.3 \mathrm{ppm}$.

\subsubsection{Evidence of Evolutionary Relationship between M. liflandii and M. marinum}

M. liflandii is a frog pathogen that was first isolated in 2004 from infected laboratory frogs Xenopus tropicalis and X. laevis as reported by Trott et al. ${ }^{254,255}$ At that time, it was suggested that $M$. liflandii was closely related to either M. marinum or M. ulcerans. ${ }^{254}$ When the lipid profile of this species was compared with the profiles of other Mycobacteria (M. tuberculosis, M. smegmatis, M. ulcerans, and M. marinum), indeed, the similarity between the M. liflandii lipid profile and those of $M$. ulcerans and M. marinum was evident by the production of abundant PDIM, mycolatone and phenolic glycolipids. In fact, $M$. liflandii lipid profile represented an intermediate lipid expression between $M$. marinum, which produces abundant glycolipids and carotenoids, and M. ulcerans, which lacks most of these molecules (Figure 3.4a-c). This observation was validated recently through in-depth genomic study of several mycolactone-producing strains. ${ }^{256}$ That study suggested a recent reductive evolution of $M$. ulcerans from M. marinum, whereas other mycolactone-producing strains like $M$. liflandii were identified as an earlier divergence during this evolutionary process. ${ }^{257,258}$

From this study, it was also revealed that $M$. liflandii produces significant amounts of PGL, a class of lipids closely related to phthiocerol dimycocerosates, which are produced by few pathogenic mycobacterial strains. ${ }^{259-261}$ Signals attributed to PGL from $M$. liflandii were found to superimpose with signals corresponding to PGL in the $M$. marinum spectrum. ${ }^{262}$ This observation was confirmed by the subsequent isolation and structural characterization of this molecule which was found to be phenolphthiodiolone glycosylated with 3-O-methyl-rhamnose at the phenolic hydroxyl (Figure 3.4d). This supports our previous observation about the evolutionary relationship between the three species. On one hand, M. liflandii has a limited lipid metabolome like that of M. ulcerans and on the other it still can produce PGL while M. ulcerans can not.

\subsubsection{Different Lipid Profiles for Different Laboratory Strains and Clinical Isolates}

Different laboratory strains of $M$. tuberculosis are traditionally used in TB research either for drug susceptibility testing or other microbiological and biochemical research. In this experiment, the avirulent $M$. tuberculosis $\mathrm{H}_{37} \mathrm{Ra}$ and some virulent laboratory strains of $\mathrm{H}_{37} \mathrm{Rv}$, Erdman's, the highly virulent HN878 strain and the highly transmittable CDC1551 strain were used. Comparison between these strains were limited only to their lipid profiles as it is not possible to obtain an HR-MAS profile of such virulent strains due to biosafety concerns. Some subtle differences, most of them are quantitative in 

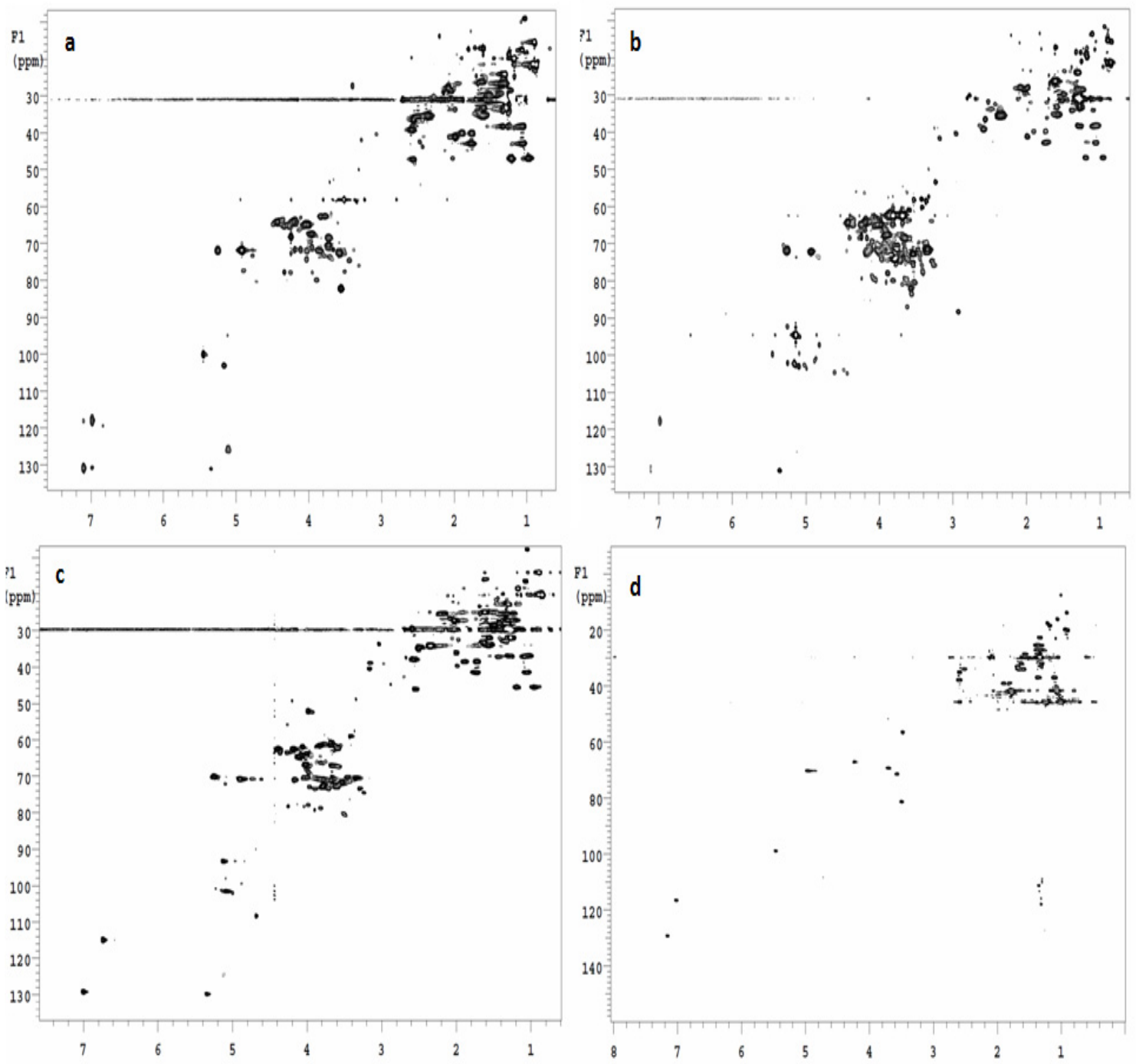

Figure 3.4 Lipidomics Characterization of M. liflandii

a-C, ${ }^{1} \mathrm{H}^{13}{ }^{13} \mathrm{C}$ HSQC spectra $\left(\delta^{1} \mathrm{H}, 0.6-7.6 \mathrm{ppm}, \delta^{13} \mathrm{C} 14-136 \mathrm{ppm}\right)$ of total lipid extract from M. liflandii, (a), M. marinum, (b), and M. ulcerans, (c). M. liflandii is thought to represent an intermediate stage in the reductive evolution of M. ulcerans from M. marinum progenitor which is reflected in these lipid profiles by comparing the diversity of the glycolipid pools of the three species. (d), ${ }^{1} \mathrm{H}^{13}{ }^{13} \mathrm{C}$ HSQC spectrum of the major PGL purified from M. liflandii total lipid extract. 
nature, were observable between different strains (Figures B.22-B.26). For example, when compared to M. tuberculosis $\mathrm{H}_{37} \mathrm{Rv}$, Erdman's strain seemed to produce higher levels of methyl-branched fatty acids while $\mathrm{H}_{37} \mathrm{Ra}$ produced significantly lower amounts. The validation of these observations is beyond the scope of this study and without prior reports of similar results they could not be further verified.

However, our observations on strains from well characterized lineage such as HN878 strain which belongs to the $\mathrm{W}$-Beijing lineage can be easily verified. In the lipid profile of HN878, both qualitative and quantitative differences were observed. As an example of the first, the production of PGL was detected in HN878 lipid profile by the presence of its two aromatic signals at $\delta^{1} \mathrm{H}=7.02,{ }^{13} \mathrm{C}=17.6$ and $\delta^{1} \mathrm{H}=7.18,{ }^{13} \mathrm{C}=129.7 \mathrm{ppm}$ and its $\mathrm{CH}_{2}$ benzylic protons at $\delta^{1} \mathrm{H}=2.58, \delta^{13} \mathrm{C}=46.8 \mathrm{ppm}$ (Figure B.25). As an example of the quantitative differences, we reported the production of unusually high levels of triglycerides as indicated by the high intensity of the signal of C-2 glycerol at $\delta^{1} \mathrm{H}=5.30$, $\delta^{13} \mathrm{C}=69.32$ which comes in agreement with recently published data that characterized the general features of the W-Beijing lineage. ${ }^{263}$

\subsubsection{Lipid Profiles of Other Bacteria: Corynebacterium and Bacillus}

Corynebacteria are mycolic acid producing actinomycetes and they share a similar cell wall structure with Mycobacteria that contains polysaccharides complexes like mAG and LAM as well as free lipids such as acylated trehalose and acylated glucose. ${ }^{264}$ The HRMAS of C. glutamicum showed a similar AG and LAM structures to that observed in most Mycobacteria species except for the apparent absence of the 3,5- $\alpha$-Ara $f$ anomeric signal which is usually reported at $\delta^{1} \mathrm{H}=5.07, \delta^{13} \mathrm{C}=107.38$ as it is the case of M. bovis BCG (Figure 3.5a,b). Instead, two other anomeric signals were detected in $C$. glutamicum spectrum at $\delta^{1} \mathrm{H}=5.08, \delta^{13} \mathrm{C}=109.25$ and at $\delta^{1} \mathrm{H}=5.01, \delta^{13} \mathrm{C}=105.74 \mathrm{ppm}$. This shift indicates a significant variation in the branching patterns of the $\mathrm{AG}$ macromolecule between Mycobacteria and Corynebacteria. There was no literature report of such observation to the best of our knowledge. Upon analysis of cell total lipids, the lipid profile of $C$. glutamicum displayed similar general features to that of Mycobaceria. Abundant glycolipids, phospholipids, mycobactins and limited amount of triacyl glycerols were easily detected (Figure 3.6).

The second step was to utilize the method for the analysis of completely unrelated species. Two Gram positive species were chosen; $B$. anthracis Sterne, a vaccine strain of the pathogenic $B$. anthracis, and B. subtilis, a soil bacteria. Both species displayed a lipid profiles that was different to a large degree from that of Mycobacteria and Corynebacteria. The only reported similarities were in the fatty acid and some phospholipids signals which are present in all bacterial plasma membranes. A distinct feature observed in these two profiles was the presence of two anomeric proton signals at $\delta^{1} \mathrm{H}=4.39, \delta^{13} \mathrm{C}=102.9$ and ${ }^{1} \mathrm{H}=4.32,{ }^{13} \mathrm{C}=103.42$ (Figure 3.7). These signals are likely to be related to the mitogenic glycerolphosphoglycolipids isolated by Fischer et al in $1978^{265}$ and later confirmed in its structure and mitogenicity by $\mathrm{Li}, \mathrm{Y}$ and colleagues. ${ }^{266}$ On comparison between the two species, $B$. anthracis lipid profile was rich with signals 

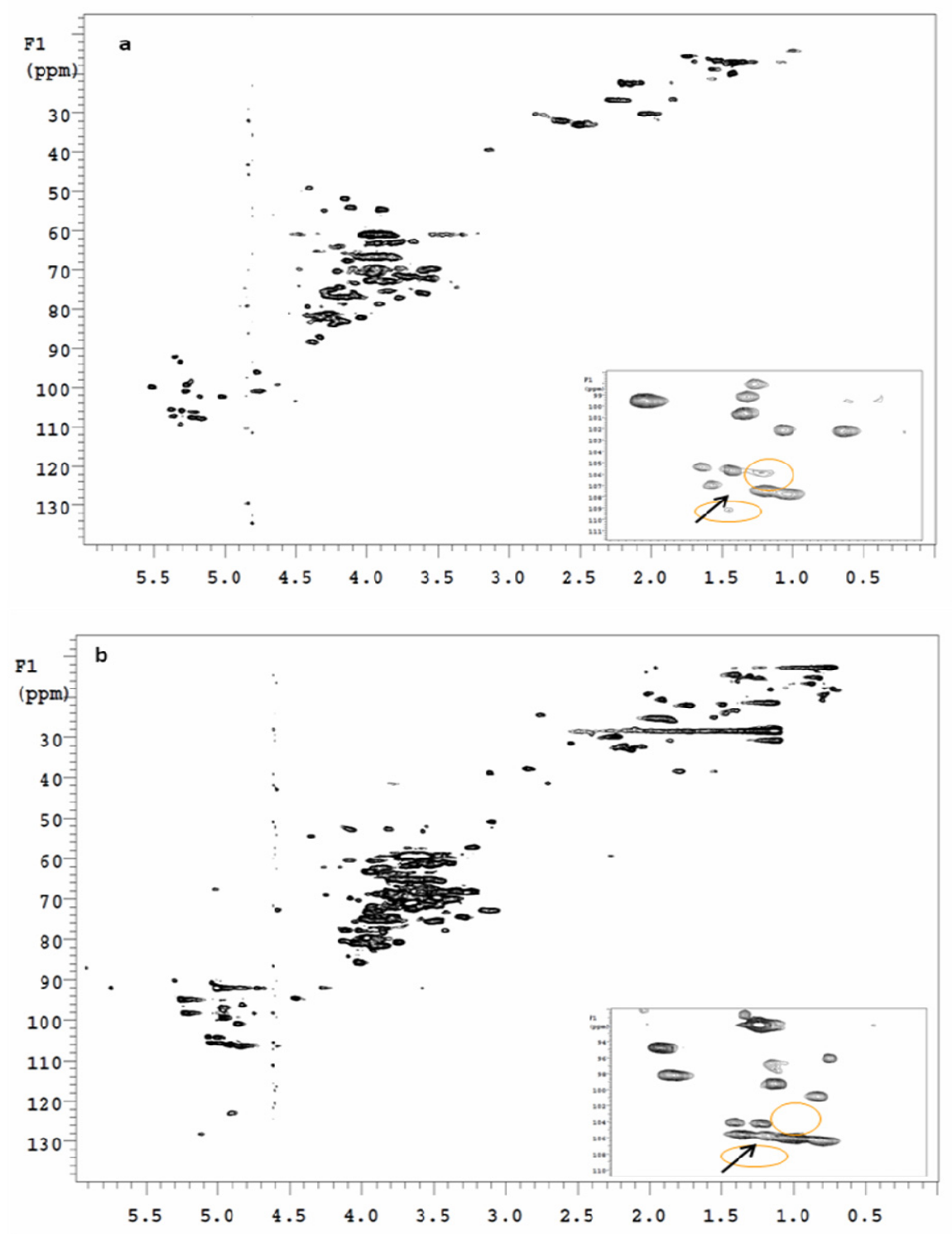

Figure 3.5 Comparison between the HR-MAS Profile of C. glutamicum and M. bovis BCG

HR-MAS profiles of C. glutamicum, (a), and M. bovis BCG, (b). Differences can be observed in the AG structure of $C$. glutamicum with the appearance of two distinct signals at $\delta^{1} \mathrm{H}=5.08, \delta^{13} \mathrm{C}=109.25$ and $\delta^{1} \mathrm{H}=5.01, \delta^{13} \mathrm{C}=105.74 \mathrm{ppm}$ (signals are circled in the blow-up inserts). The anomeric signal for 3,5- $\alpha$-Ara $f$ detected at $\delta^{1} \mathrm{H}=5.06$, $\delta^{13} \mathrm{C}=107.04 \mathrm{ppm}$ in the HR-MAS of $M$. bovis BCG was absent from the HR-MAS spectrum of $C$. glutamicum as indicated in the inserts by the black arrow. 


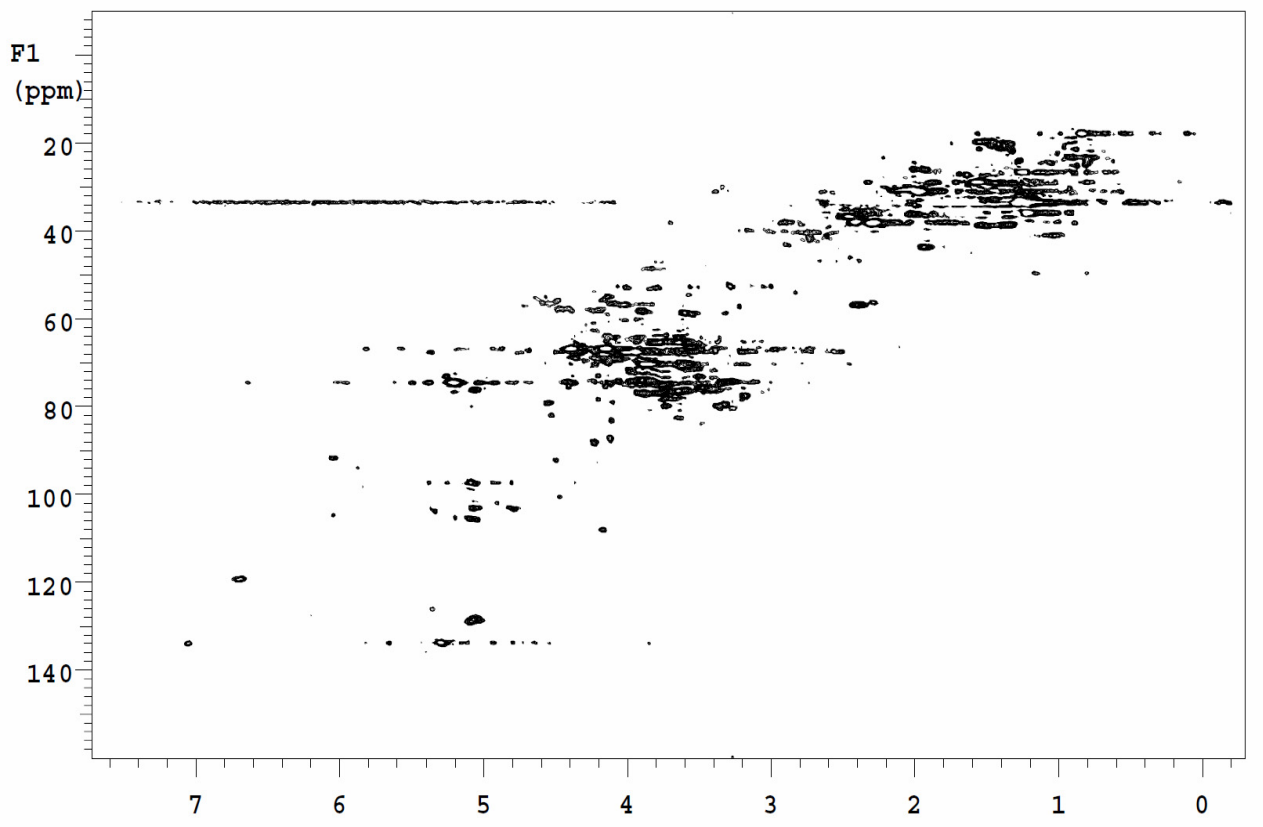

Figure 3.6 2D-HSQC Lipid Profile of C. glutamicum
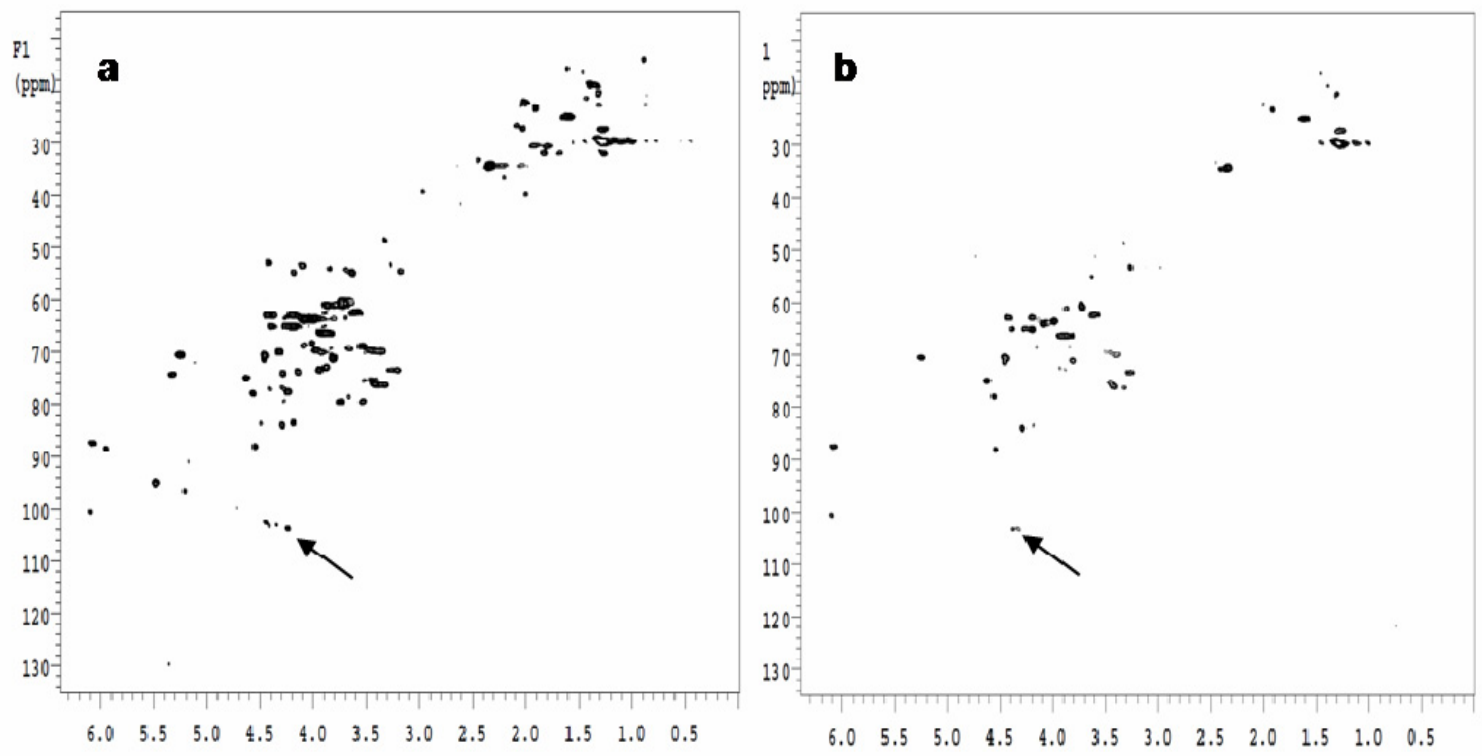

Figure 3.7 2D-HSQC Lipid Profile of Bacillus Species

2D-HSQC lipid profile of B. anthracis (Sterne), (a), and B. subtilis, (b). The arrows indicate the position of the two anomeric signals of Bacillus mitogenic glycerol phosphoglycolipid at $\delta^{1} \mathrm{H}=4.39,{ }^{13} \mathrm{C}=102.9$ and $\delta^{1} \mathrm{H}=4.32,{ }^{13} \mathrm{C}=103.42 \mathrm{ppm}$. 
for long aliphatic chains, sugar residues and unsaturated substructures, while that of $B$. subtilis displayed smaller number of signals especially in the sugar residue regions and very weak signals in the oleifenic region (Figure 3.7).

\subsection{Conclusion}

\section{4.1 NMR-Based Approach for Comprehensive Cell Wall Analysis}

A combination approach of HR-MAS monitoring of cell wall polysaccharides (mainly AG and LAM) and 2D-HSQC lipid profiling was developed. Such approach can provide researchers with valuable insights to the structural features of the mycobacterial cell wall in relatively short time and with reasonable degree of confidence in data interpretation. Based on the examples discussed in this chapter and in the previous chapter, a cell culture of 30-40 milliliters enriched with ${ }^{13} \mathrm{C}$-dextrose and/or glycerol can be used to produce comprehensive reproducible profiles for both cell lipids and polysaccharides. Sample preparation protocols for both techniques follow the same set of procedures for the most part and human effort designated for this purpose can be greatly reduced as a result. The two techniques combined require the use of a single high field NMR spectrometer with interchangeable sample probes to accommodate both solid (HR-MAS) and liquid sample (lipid profile).

This combination in itself can also serve the purpose of a self control. For example, when certain gene mutation is being sought to affect cell wall lipid biosynthesis, lipid extract from the mutant strains can be analyzed by 2D-HSQC NMR for that purpose. Meanwhile, the same cells that were extracted can be used to generate an HR-MAS profile to confirm the integrity of other cell wall components such as AG and LAM. Such approach provides solidity of the interpreted data and can also help identify some important cellular process like adaptation to gene mutation and possible metabolic coupling.

\subsubsection{Species Differentiation Using NMR Metabolic Profiling}

Mycobacteria species and strains can be differentiated by a wide array of methods with very high sensitivity. The purpose of this study was not to offer an alternative to such techniques. However, several goals have been achieved from such study including; i) demonstrating the utility of this combined approach across different number of Mycobacteria that are representatives of different clades, ii) validating the utility of the technique by its capability to detect both qualitative and quantitative differences between multiple species, iii) starting a database of standardized referenced lipid profiles of several Mycobacteria species and strains that can later be used as base line references, iv) providing information that can help in the assignment of biomarkers signals for some lipid molecules by choosing the most suitable lipid profiles where these signals can be observed in high abundance and with optimal resolvability, v) exploring the possibility of application of these method to characterize the main features of the cell wall 
compositions of newly isolated species and vi) pinpointing to differences that exist between different laboratory strains and clinical isolates. Such differences must be taken into consideration when comparing and using research data obtained through the use of different laboratory strains.

\subsubsection{Applicability to Other Bacterial Species}

Like Mycobacteria, all actinomycetal line (Mycobacteria, Corynbacteria, Nocardia and Rhodococci) have a chemotype IV cell wall and possess a complex cell wall structure rich in lipids especially mycolic acids. The combined NMR approach discussed above can be helpful in studying a large array of these species as long as they can be maintained in the laboratory in a well-defined growth media that uses glycerol and/or dextrose as the primary carbon source. As a demonstrative example, C. glutamicum was grown in BHI media where ${ }^{13} \mathrm{C}_{6}$-dextrose was added as the primary carbon source. This isotope enrichment strategy was successful in providing high quality HR-MAS and lipid profiles as outlined in section 3.3.7. Outside the actinomycetals line, all eubacteria share a similar phospholipids bilayer plasma membrane and through lipid profiling, components of these membranes can be investigated and compared with relative ease. This concept was used here to compare between two Bacillus species. Such results also underscore the applicability of such methodology to a wide spectrum of species and in diverse research fields. 


\section{CHAPTER 4. METABOLOMIC STUDIES OF MYCOBACTERIA ADAPTATION TO STRESS CONDITIONS}

\subsection{Introduction}

Since the introduction of streptomycin as the first treatment for TB in the 1940s, we have made a significant progress that is undeniable. The great white plague that devastated Europe is now a curable disease. However, this curable disease is reported in nine million cases every year and is claiming close to two million lives annually. ${ }^{12}$ For a certain period of time we were very optimistic of our capability to reduce TB to "a minor aliment to man" as Waksman once announced in his 1960s book "The Conquest of Tuberculosis." ${ }^{267}$ This optimistic prediction seems so far from reality now like never before. We now live in a world where one third of the population harbor the tubercle bacilli in their bodies and where there is a serious concern in our ability to treat highly drug resistant forms of the disease.

Our failure to eradicate TB is caused by the success of the tubercle bacilli to tolerate anti-TB chemotherapy and develop resistance. Unlike most other bacterial infections that can be treated with a single antibiotic, monotherapy has not been reliable as an anti-TB treatment. Currently, any effective anti-TB regimen is based on the use of combination therapy to deter the rise of resistance. ${ }^{34}$ However, one of our major health concerns now is the global spread of MDR-TB. Although MDR-TB is currently treatable through a two years regimen that includes several second line anti-TB drugs, it is hard for TB patients to adhere to such lengthy and often not well tolerated course. As a result of our failure to control MDR-TB, we were confronted with XDR-TB, a form of the disease that resists treatment with rifampin and INH in addition to key second line drugs (a fluroquinolone and at least one of the injectable aminoglycosides). ${ }^{36,268}$ XDR-TB is associated with very high mortality rates and is currently untreatable by available anti-TB chemotherapy. ${ }^{36}$

The tolerance of $M$. tuberculosis to most antibacterial chemotherapy and the rapid rise of resistance against the few useful anti-TB drugs can be partially explained by the unique characteristics of the tubercle bacilli and the pathological features of TB itself. $M$. tuberculosis cell wall displays unique complexity which includes an outer lipid membrane of mycolic acids that works as an efficient permeability barrier against the entry of most chemotherapeutic agents. Inside their host, the infecting bacilli exist in different metabolic states including actively dividing and latent bacilli. ${ }^{217,218}$ While actively growing bacteria are susceptible to commonly used anti-TB drugs, latent bacilli are much harder to kill due to their slow metabolic rates and limited replication. ${ }^{52}$ In addition, these latent bacilli are enclosed inside the TB-granuloma, a hypoxic locus that receives limited blood supply. To constantly deliver anti-TB drugs at their inhibitory concentration inside the TB-granuloma, anti-TB drug dosage and administration have to be carefully monitored. Failure to maintain proper concentration of the drug inside the granuloma enriches the latent population with naturally occurring resistant mutants. When a patient prematurely terminates his TB treatment course due to a temporary relief of symptoms, the latent bacteria can remerge causing a recurrence of the disease. The 
pursuit of novel chemotherapeutic agents that target the latent bacilli is now a top priority in the TB research community. ${ }^{198,269,270}$ Such development when it happens will be a major breakthrough as it should shorten the treatment course and limit the incidence of resistance. In order to develop such drugs, we need to identify the intrinsic factors that contribute to Mycobacteria latency and hence the persistence of the tubercle bacilli.

Several laboratory models have been developed to study the metabolic states of the different bacterial population during latency. The most common animal models include the murine, Guinea pigs, rabbits and non human primates. ${ }^{52}$ Although the murine model is the most widely used in research, it carries the least similarities to the pathological features of the human disease. ${ }^{52}$ On the other hand, non human primate models develop lung tuberculosis that is very similar to human TB but their use in routine research is limited by their high cost. ${ }^{271,272}$ In vitro latency models that try to mimic the growth conditions of the latent bacilli have also been developed. The most common is that developed by Wayne in 1996 which uses sealed culture with limited aeration to drive the bacterial to a state of dormancy through gradual oxygen depletion. ${ }^{273,274}$ Bacilli grown under these conditions pass through two phases of non replicating growth where cells prepare for and then enter a microaerophilic metabolic phase. ${ }^{274}$ To adapt to these hypoxic conditions, several genes are either down regulated or up-regulated to transfer the cells to a state of low energy requirement. Lower expression of type-I NADH dehydrogenase and most subunits of ATP synthase have been reported, ${ }^{275,276}$ while isocitrate lyase, ${ }^{277}$ triglyceride synthases, ${ }^{278}$ and nitrate reductases ${ }^{275}$ are among genes that have been found to be upregulated.

Other in vitro models are designed to test the persistence of Mycobacteria in case of nutrient starvation. $M$. tuberculosis has the ability to survive and replicate inside human macrophages and within the granuloma structure in an environment with a limited supply of nutrients. In these models, a population of persistent drug tolerant bacilli can be developed which can be used to validate the ability of drugs to kill persistent bacilli. ${ }^{220}$ The response of Mycobacteria under nutrient starvation includes down regulation in the biosynthesis of most cell metabolites with up-regulation in the transcription of genes involved in RNA synthesis and modification. ${ }^{279,280}$ Cells maintained under these nutrient starvation conditions show significant stress resistance, higher stability in their mRNA and low susceptibility to common anti-TB drugs such as rifampin and INH. ${ }^{279,281}$

Inside the macrophage, not only does $M$. tuberculosis have to survive in limited nutrient supply but it also has to endure abundance of reactive oxygen species and nitrogen intermediates in an environment of low $\mathrm{pH}^{57,241,282}$ In the laboratory, the response of Mycobacteria to stress condition (stringent response) can be induced by starvation as well as by exposure to acids or UV lights. ${ }^{280}$ Evidence suggested that significant number of genes that are up-regulated under laboratory stress conditions were also identified in separate studies as essential for mycobacterial in vivo survival. ${ }^{52,217,277}$ These findings validate the use of in vitro models to identify genes and metabolic products that are essential for Mycobacteria infectivity. 
This report investigates the changes of the mycobacterial metabolome under several stress conditions mainly low oxygen, limited supply of nutrients and total starvation in phosphate buffer saline (PBS). M. bovis BCG, a vaccine strain for TB, is used here as a surrogate for M. tuberculosis. This use is common due to the availability of a complete genome sequences and the reported similarity between the two species.

\subsection{Materials and Methods}

\subsubsection{Strains and Growth Conditions}

Mycobacteria bovis BCG was inoculated at an initial O. $\mathrm{D}_{\lambda 600}$ of 0.05 in Middlebrook $7 \mathrm{H} 9$ growth media supplemented with $\mathrm{ADG}$ and $0.05 \%$ Tween 80 . For ${ }^{13} \mathrm{C}$-enrichement the dextrose and glycerol in the ADG supplement were replaced by ${ }^{13} \mathrm{C}_{6}$-dextrose and ${ }^{13} \mathrm{C}_{3}$-Glycerol respectively (Cambridge Isotope Lab. MA). For low nutrients conditions, the concentration of glucose and glycerols were reduced to $0.02 \%$ instead of the normal $0.2 \%$ concentration obtained using the ADG supplement while all other growth conditions were maintained unchanged. All bacterial cultures were incubated at $37^{\circ} \mathrm{C}$ with continuous shaking at $250 \mathrm{rpm}$.

\subsubsection{Starvation Model}

Forty milliliters of M. bovis BCG grown in ${ }^{13} \mathrm{C}$-enriched Middlebrook $7 \mathrm{H} 9$ to the mid exponential phase $\left(O . D_{\lambda 600}=0.6\right)$ were harvested by centrifugation then washed twice with $10 \mathrm{mls}$ of PBS. After washing, cells were left to starve in $40 \mathrm{mls}$ of PBS without shaking at $37^{\circ} \mathrm{C}$ for previously determined time periods. For reactivation of the starved cells, cells were harvested by centrifugation at $3700 x g$. The cell pellet was then washed twice with $10 \mathrm{mls}$ of plain $7 \mathrm{H} 9$ then re-suspended in ${ }^{13} \mathrm{C}$-enriched $7 \mathrm{H} 9$. Viable count of the starved and reactivated cells was determined by plating dilutions of the cell culture on Middlebrook 7H11 agar plates and the number of colonies was counted after 4 weeks incubation period at $37^{\circ} \mathrm{C}$.

\subsubsection{Low Oxygen Model}

$200 \mathrm{mls}$ of Middlebrook $7 \mathrm{H} 9$ supplemented with ${ }^{13} \mathrm{C}_{3}$-glycerol and ${ }^{13} \mathrm{C}_{6}$-dextrose were inoculated with M. bovis BCG to an O.D $\mathrm{\lambda}_{2600}$ of 0.005 in round bottom glass flasks with $300 \mathrm{ml}$ total capacity. The culture flasks were sealed with silicon rubber caps and were incubated at $37^{\circ} \mathrm{C}$ with slow stirring at $100 \mathrm{rpm}$. At different time points, the optical density of the bacterial culture was measured and cells were subsequently harvested. Methylene blue was used as a redox indicator to monitor oxygen level at $15 \mu \mathrm{M}$ concentration. For reactivation, the bacterial cultures were transferred to new flask with $5 \mathrm{X}$ head space with continuous shaking at $250 \mathrm{rpm}$ at $37^{\circ} \mathrm{C}$. 


\subsubsection{Sample Preparation for HR-MAS}

Cells were harvested by centrifugation at $3700 \mathrm{xg}$ for 10 minutes. The cell pellet was washed twice with $5 \mathrm{mls}$ of $\mathrm{D}_{2} \mathrm{O}$. The supernatant washing liquid was removed in between by centrifugation at $3700 \mathrm{xg}$ for 10 minutes. The washed pellet was then re-suspended in a minimal volume of $\mathrm{D}_{2} \mathrm{O}$ and $40 \mu \mathrm{l}$ of the cell suspension was transferred to an NMRMAS rotor.

\subsubsection{Lipid Extraction}

The bulk of the previously washed cell pellet was transferred to a pre-weighed microcentrifuge tube using one $\mathrm{ml}$ of $\mathrm{D}_{2} \mathrm{O}$. After removal of $\mathrm{D}_{2} \mathrm{O}$ by centrifugation, cells were re-suspended ( $3 \mu \mathrm{l}$ per $1 \mu \mathrm{g}$ wet cell weight) in $2: 1$ mixture of $\mathrm{CDCl}_{3}: \mathrm{CD}_{3} \mathrm{OD}$ to which DMSO was added as an internal standard $(0.0025 \mu$ per every milligram of cells). Lipids were extracted by continuous shaking for 90 minutes at $37^{\circ} \mathrm{C}$ degrees. After extraction, additional volume of $\mathrm{CD}_{3} \mathrm{OD}$ was added $(1 \mu \mathrm{l}$ per $1 \mathrm{mg}$ of original cell weight) to achieve a single phase solution. The final lipid extract was removed by centrifugation for 10 minutes at 21,000xg then transferred to 5mm NMR tube.

For mycolic acid saponification, two milliliters of $1 \mathrm{M}$ methanolic $\mathrm{KOH}$ were added to the delipidated cells in glass screw cap tubes. Saponification process was continued for 16 hours with continuous stirring. After saponification the solution was cooled down and then neutralized with concentrated $\mathrm{HCl}$ to $\mathrm{pH}=4$. Free mycolic acids were then extracted twice with diethyl ether. The combined extract was washed with deionized water and then dried with rota-evaporator under vacuum at $40^{\circ} \mathrm{C}$. For NMR acquisition, dried mycolic acids were dissolved in 1:1 $\mathrm{CD}_{3} \mathrm{OD}: \mathrm{CDCl}_{3}$.

\subsubsection{NMR Acquisition and Data Processing}

2D-HSQC lipid profiles were obtained using a Varian Inova-500MHz NMR spectrometer equipped with a $5 \mathrm{~mm}$ trpfg probe using $2 \mathrm{D}-\mathrm{HSQC}$ pulse sequence $(\mathrm{nt}=10$ $\mathrm{ni}=256$ ). Samples were individually shimmed, locked and tuned at $24^{\circ} \mathrm{C}$ and other acquisition parameters were optimized for each sample. NMR data was processed using Felix-NMR. Specific cross peaks in the 2D-NMR spectrum were integrated and normalized to the integration value of the DMSO peak that was used as an internal standard. For acquisition of HR-MAS spectra, MAS rotors were inserted at the magic angle in a GHx-nanoprobe with continuous spinning at $2 \mathrm{KHz}$ and temperature of $24^{\circ} \mathrm{C}$ degrees. Acquisition parameters were adjusted for individual samples. 2D HSQC pulse sequence was applied for 8 transients and 256 increments. 


\subsection{Results}

\subsubsection{Effect of Low Nutrients}

Cells grown in Middlebrook $7 \mathrm{H} 9$ with $0.02 \%$ only of dextrose and glycerol showed normal growth curve compared to that of cells grown with $0.2 \%$ glucose and glycerol. However, when the O.D. reached a value of 0.4-0.6, the growth of cells grown in low nutrient supply halted. The lipid profile of these partially starved cells looked overall less crowded than that of cells grown under normal growth conditions. The quality of the spectra was first assessed by referring to the signals of cell membrane phospholipids which fell in the normal range observed in control experiments. Upon comparison of these lipid profiles, lower intensities of signals of major acylated trehalose and triacyl glycerols were observed. There was also a significant reduction in the levels of unsaturated fatty acids and $\mathrm{PIM}_{6}$ when compared to the levels recorded in normally grown cells that have been harvested at different O.D. (Figure 4.1).

Upon examining the HR-MAS spectra for changes in the mAG complex, no such changes were readily detectable (Figure 4.2). However, signals corresponding to the cell wall glucan were detected at a relatively low intensity when compared to cells grown under normal growth conditions and harvested at different stages of their growth. LAM signals were recorded at significantly lower intensity in the partially starved cells as opposed to AG signals which remained at stable levels. Meanwhile, all lipid signals that could be detected in the HR-MAS spectrum were either absent (such as unsaturated fatty acids signals) or recorded at much lower intensity (Figure 4.2).

\subsubsection{Effect of Starvation}

Cells starved in PBS were maintained over a period of four weeks. Over this period, we observed a drop in the viable count of the starved cells by approximately one logarithmic unit (Figure 4.3a). This loss in cell viability was directly detected in the cellular lipid profile as one logarithmic unit decrease in the intensity of NMR cross peaks previously assigned to cellular phosphoglycerolipids (PL) as shown in Figure 4.3b. Other cellular lipids, most notably triacyl glycerols (TAG), were greatly affected by prolonged starvation. TAGs are associated with the cell wall lipid pseudomembrane but they are mainly located inside the cytoplasm in large lipid vacuoles. After starvation for two days, the TAG pool was depleted from the cells (based on the detection limit of this method), while other cellular components remained at their original levels. However, after only six hours of transferring the cells to nutrients rich media, TAG levels were measured at twice their value and continued to rise after 24 and 48 hours. During that time, cells seemed to be preparing for a complete recovery and cell replication was halted. After 2-4 days of this peak, the TAG levels were restored back to their normal abundance in $M$. bovis BCG (Figure 4.3c).

Other cell wall lipids (PDIM, fatty acids, TDM, PIM and AT) of the cell wall remained at their original levels for more than two weeks but a significant drop in their 


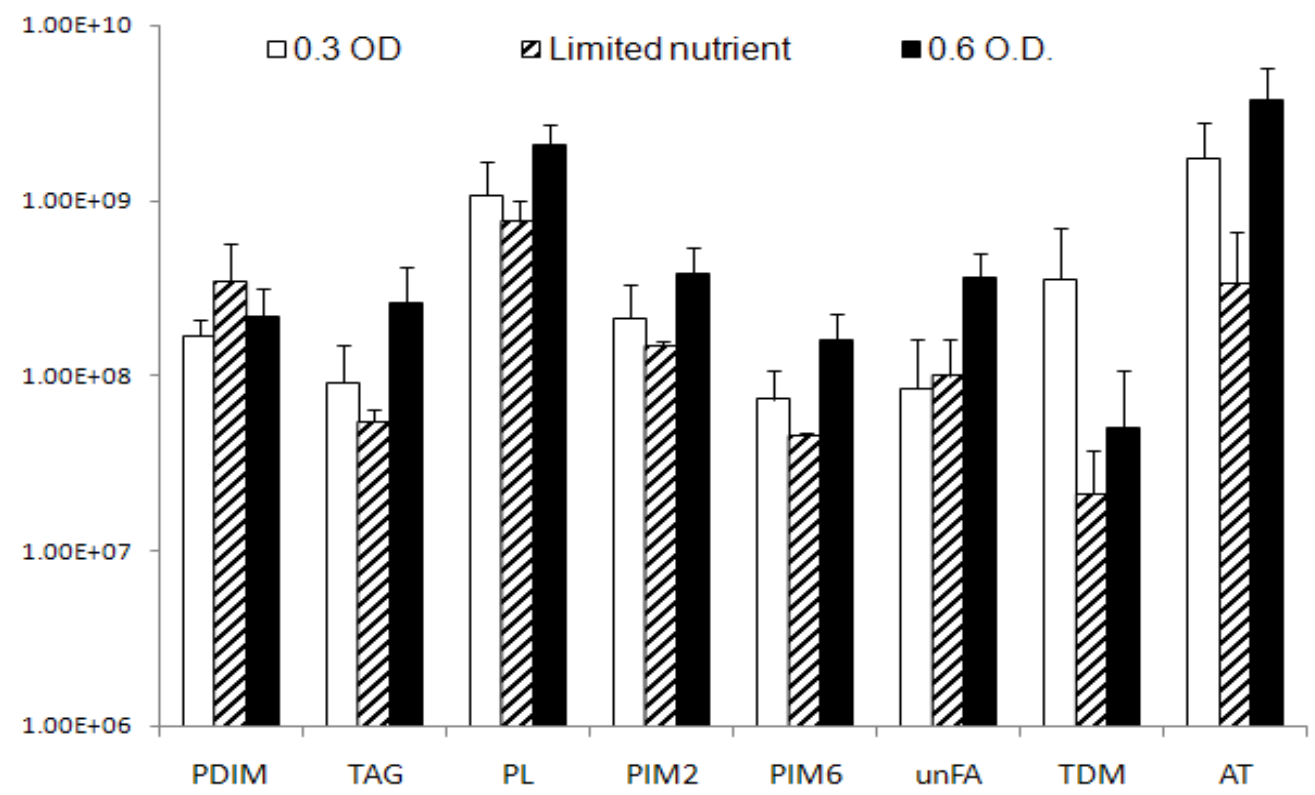

Figure 4.1 Effect of Limited Nutrients Supply on the Cellular Lipid Content of M. bovis BCG

Cellular lipid contents are expressed as the integration volumes of specific biomarker signals for each lipid class; $\delta \mathrm{H}=1.23, \delta \mathrm{C}=45.54 \mathrm{ppm}$ for $\mathrm{PDIM}, \delta \mathrm{H}=5.30, \delta \mathrm{C}=69.31 \mathrm{ppm}$ for TAG, $\delta \mathrm{H}=5.26, \delta \mathrm{C}=70.74 \mathrm{ppm}$ for $\mathrm{PL}, \delta \mathrm{H}=5.10, \delta \mathrm{C}=101.85 \mathrm{ppm}$ for $\mathrm{PIM}_{2}, \delta \mathrm{H}=5.01$ $\delta \mathrm{C}=102.63 \mathrm{ppm}$ for $\mathrm{PIM}_{6}, \delta \mathrm{H}=5.34, \delta \mathrm{C}=129.97 \mathrm{ppm}$ for unFA, $\delta \mathrm{H}=0.67, \delta \mathrm{C}=15.76 \mathrm{ppm}$ for TDM and $\delta \mathrm{H}=5.13, \delta \mathrm{C}=93.65 \mathrm{ppm}$ for ATs. All integral values were normalized to that of DMSO which was used as an internal standard. 
Figure 4.2 Effect of Limited Nutrients Supply on the Cell Wall AG and LAM of $M$. bovis BCG

HR-MAS 2D-HSQC spectra of M. bovis BCG cells which were grown at normal conditions and harvested at O.D $\lambda 600$ of 0.3 , (a), grown in limited nutrient supply and harvested at $\mathrm{O} . \mathrm{D}_{\lambda 600}=0.45$, (b), or grown under normal conditions and harvested at O. $\mathrm{D}_{\lambda 600}=0.6$, (c). In general, HSQC signals for cellular lipids and glucans were recorded at lower intensities in the HR-MAS spectrum of the partially starved cells as seen in (b). Shown in the bottom inserts is the variation in the abundance of LAM between the three spectra as indicated by arrows. At the same time, AG signals are recorded at comparable intensities in the three spectra (the anomeric signal of $6-\beta-\mathrm{Gal} f$ is highlighted in the three spectra inside small ovals). 

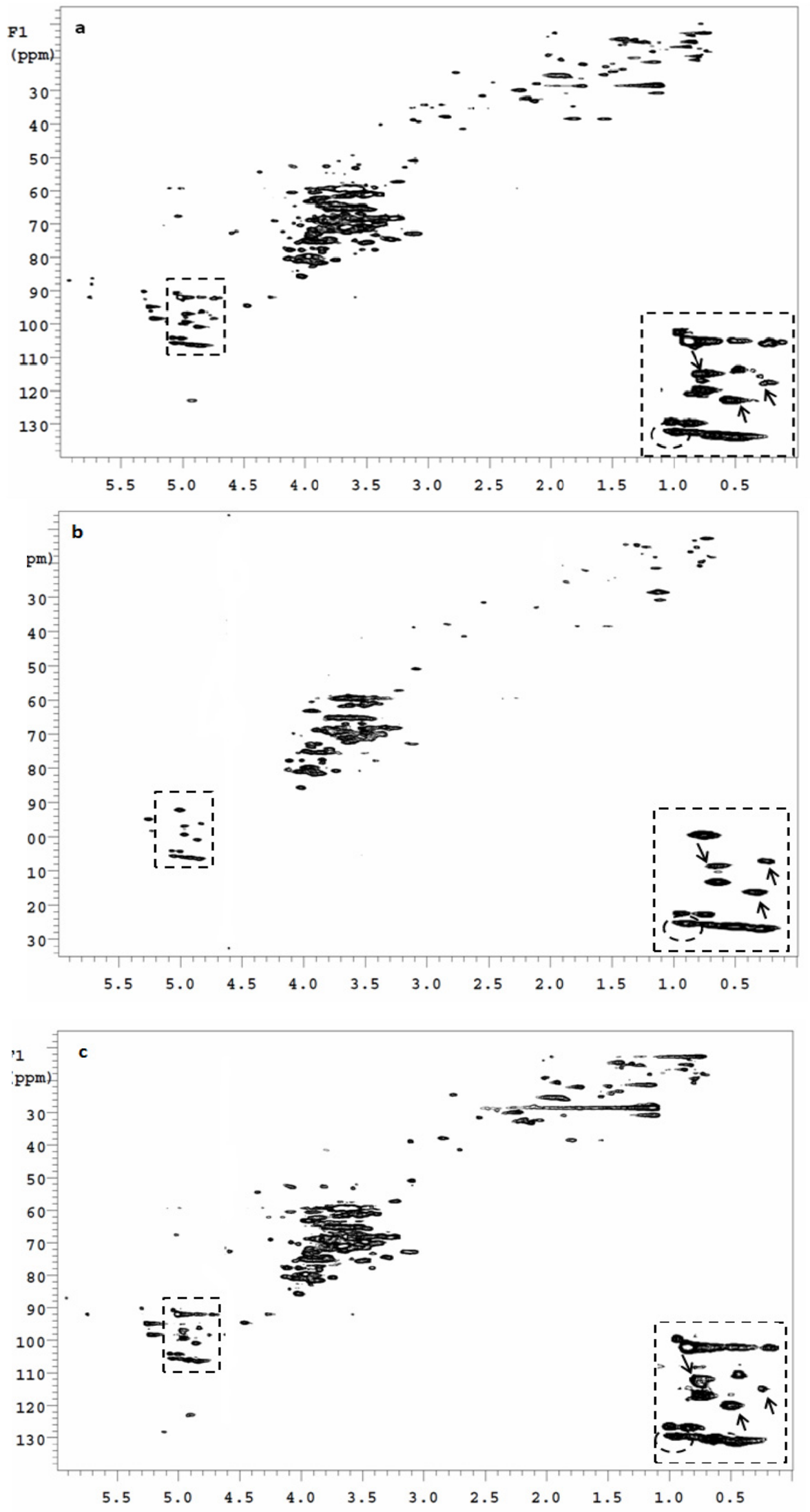
Figure 4.3 Effect of Prolonged Starvation in PBS on the Cell Wall Lipids of M. bovis BCG

A drop in the viable count of the starved cells can be observed after one week, (a), a parallel drop is also observed in cellular PL (solid diamond in b) and a less significant drop is observed in $\mathrm{PIM}_{2}$ and $\mathrm{PIM}_{6}$ content (solid square and solid triangles in b). Levels of other cell wall lipids were monitored during PBS starvation and subsequent

resuscitation, (c). All cell lipids are represented as normalized integral volumes of their corresponding biomarker signals as described under Figure 4.1. Ratios of different mycolic acid classes as compared to the total mycolate pool during starvation are plotted in (d). The relative ratios of mycolic acids were obtained as the integral value for each biomarker signal as described in Table 2.2 divided by the value of the $\beta-\mathrm{CHOH}$ signal at $\delta \mathrm{H}=3.65, \delta \mathrm{C}=72.15 \mathrm{ppm}$. 

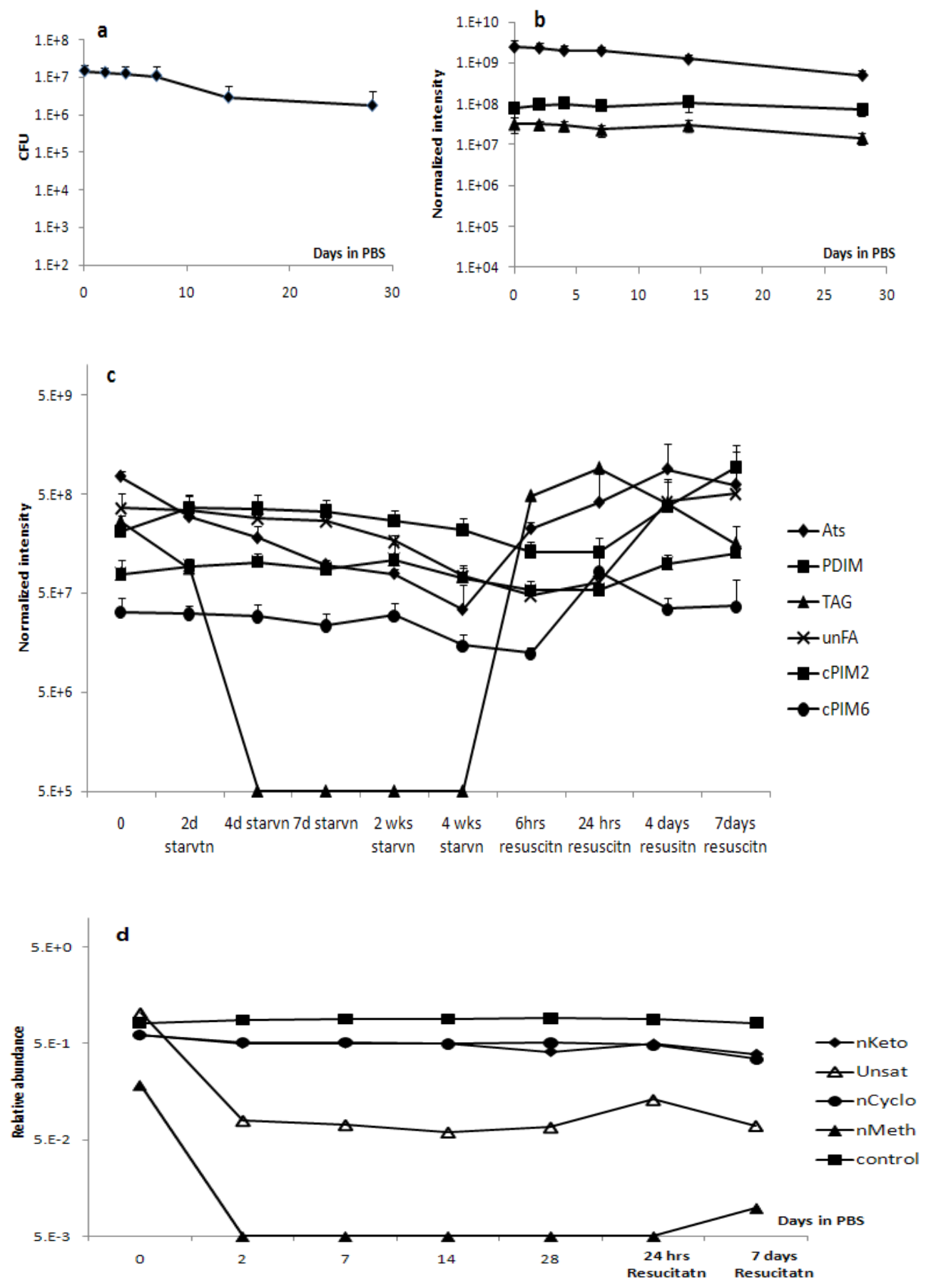
concentration was observed after four weeks of starvation (Figure 4.3c). At the same point a further drop was observed in the viable count of the starved cells. Upon transferring the starved cells to nutrient rich growth media, the cellular level of these molecules remained relatively stable for the first 24-48 hours. During the same period, the viable cell count remained constant while the concentration of TAG increased drastically. Levels detected for most cellular lipids were not restored back to their normal values till after four days of reactivation (Figure 4.3c). At which point it is expected that the bacterial population has completely shifted to a logarithmic growth phase.

In the HR-MAS profile of the starved cells, minimal changes were observed in the AG and LAM pool but there was a fast depletion of the outer cellular glucans as indicated by the gradual loss of the intensity of the glucan signals in the 2D-HSQC spectrum. Glucan signals were reported at significantly lower intensities after one week of starvation and almost disappeared after four weeks. This observation was rapidly reversed upon transferring the starved cells to a nutrient-rich media for less than 24 hours (Figure 4.4). The integrity detected in the cell wall $\mathrm{mAG}$ complex upon starvation was further confirmed by the stability observed in the covalently bound mycolic acid pool (Figure 4.3d). This suggests that even with prolonged starvation periods, Mycobacteria can preserve the integrity of its structural cell wall components with no adjustments in the substitution pattern of the covalently bound mycolic acids.

\subsubsection{Effect of Gradual Oxygen Depletion}

In our low oxygen model, $M$. bovis BCG cells exhibited a very slow growth when compared to cells grown under normal growth conditions (Figure 4.5a). Aerobic conditions were sustained in this model for the first seven days as indicated from the color stability of the redox indicator methylene blue. At the end of this period, the optical density of the cells reached 0.3 which was comparable to that reached by cells grown under normal growth condition for the same period. However, when oxygen levels started declining, the rate of cellular growth slowed down significantly. An increase in the optical density to a plateau level of $\mathrm{O} . \mathrm{D}_{\lambda 600}=0.56$ was achieved after 28 days of inoculation and after fading of the redox indicator color. This slow growth is indicative of an effective adaptation of the cells to low oxygen conditions. It has been previously reported that the success of the transfer of Mycobacteria to anaerobosis depends on the length of such transition periods.

During this slow process, some gross changes in cell density and texture were observed in the actively adapting cells that may indicate cell wall reorganization. For example, a gradual decrease in the cell density which reached its maximum at 28 days was observed. In parallel to this gross morphological change, there was a significant rise in the levels of TAG that also reached its peak at 28 days and started to decline afterwards (Figure 4.5c). Upon exposure of the cells to normal oxygen levels, normal cell density as well as TAG levels were partially restored after 6 hours. A complete restoration of cell texture and TAG levels was detected after 4-7 days (Figure 4.5c). 
Figure 4.4 Effect of Prolonged Starvation in PBS on the Cell Wall Polysaccharides of $M$. bovis $\mathrm{BCG}$

HR-MAS 2D-HSQC spectra of $M$. bovis BCG grown under normal growth conditions and harvested at $\mathrm{O} . \mathrm{D}_{\lambda 600}=0.6$, (a), M. bovis BCG starved in PBS for four weeks starting with the same optical density, (b), and $M$. bovis BCG that has been resuscitated for four days after four weeks of starvation in PBS, (c). Circled in the three spectra are signals indicative of the cell wall glucan which were greatly reduced in their intensity upon starvation as seen in (b). 

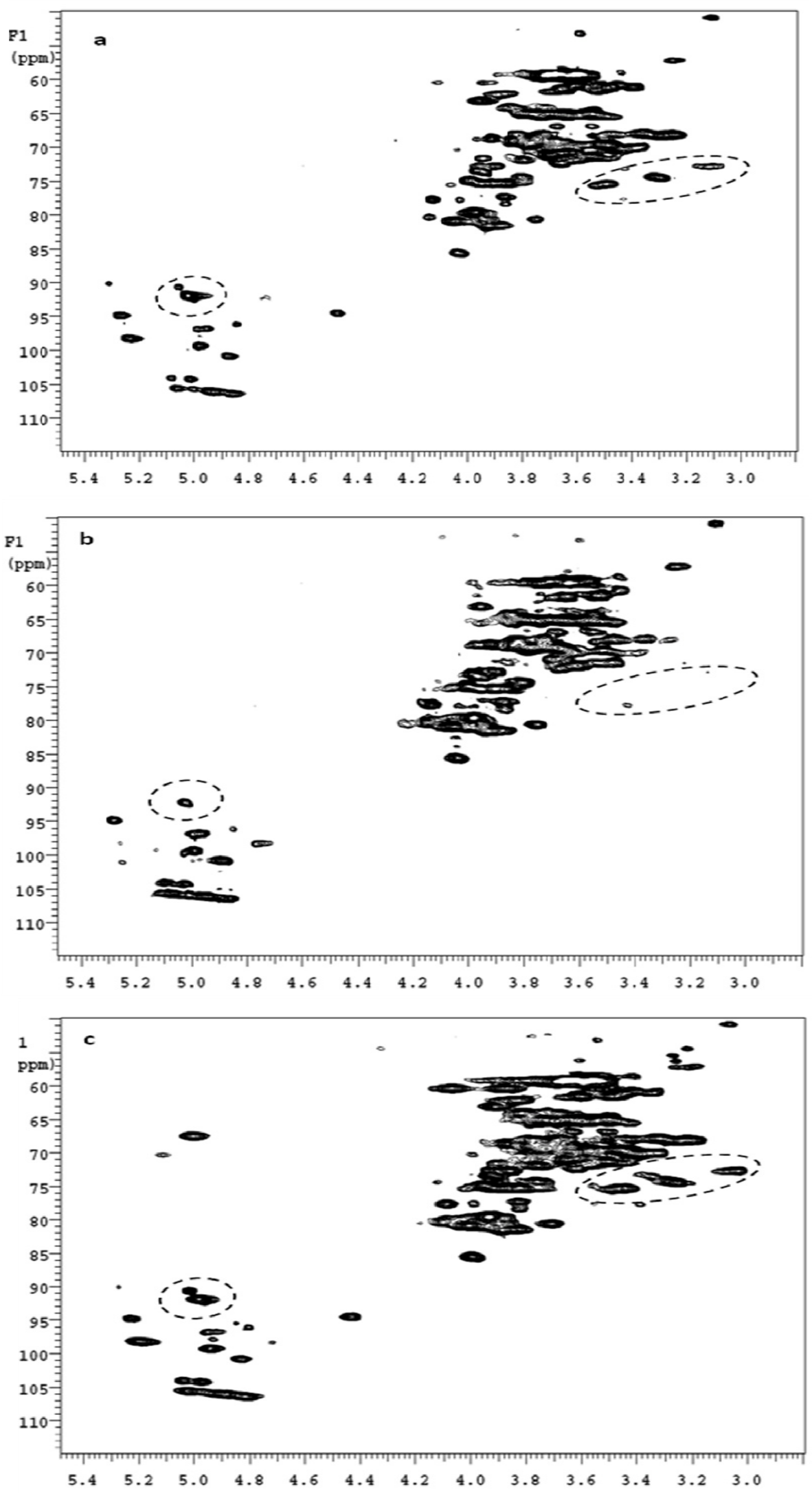

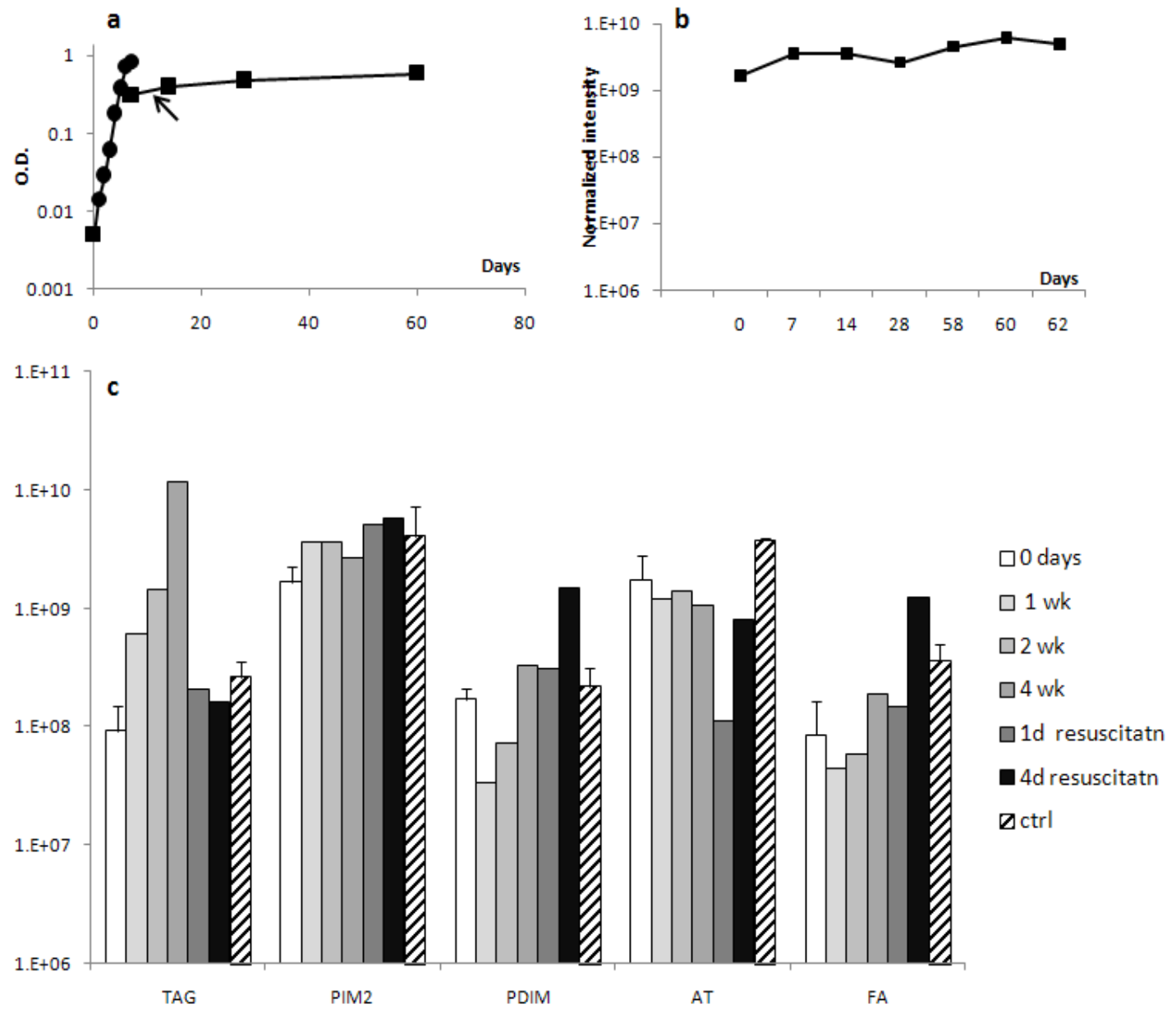

Figure 4.5 Effect of Gradual Oxygen Depletion on the Cell Wall Lipids of M. bovis BCG

M. bovis BCG cells grown under low oxygen condition exhibited a very slow growth rate as indicated by their O.D. measurement (solid square in a) when compared with cells grown under normal growth conditions (solid circles in a). The arrow indicates the time point at which the color of the redox indicator methylene blue started to fade. During the oxygen depletion process, cellular levels of membrane phospholipids remained essentially constant, (b). In panel (c), levels of other cellular lipids are reported during oxygen depletion and after resuscitation. The relative abundance of TAG, PIM2, PDIM, AT and fatty acids are plotted as the integral values of their corresponding biomarker signals normalized to that of DMSO. 
As expected, there was a remarkable stability in the levels of cellular phospholipids which levels remained unaffected during low oxygen conditions and also during reactivation by exposure to oxygen (Figure 4.5b). This stability indicates successful transformation to anaerbosis as any loss of cell viability will be detected by a loss of PL signal intensity.

In the HR-MAS spectrum, signals of TAG (which are usually recorded at low intensity in $M$. bovis BCG) were recorded at unusually high intensity indicating the presence of an overwhelming amount of these lipids. Despite that, signals of glucan and AG were easily detected at their normal levels (Figure 4.6). It is to be noted however, that a significant shift in the LAM signals was observed. Cells grown under low oxygen condition for four weeks showed very low intensity of signals indicative of 2- $\alpha$-Man $p$ and higher intensities of those indicative of 2,6- $\alpha$-Manp. As shown in Figure 4.6, the anomeric signals corresponding to 2- $\alpha$-Man $p$ and 2,6- $\alpha$-Man $p$ are usually recorded with comparable intensities (Figure 4.6a). However, under low oxygen conditions, it appears that a larger number of 2,6- $\alpha$-Man $p$ residues are present in the mannan core indicating extensive substitution of the linear mannan chain at the 2-position. Such observation can be also supported by relative increase in the intensity of signals of the terminal Man $p$ residues as detected at $\delta^{1} \mathrm{H}=4.93, \delta^{13} \mathrm{C}=102.19 \mathrm{ppm}$ and a parallel decrease in the signals for the unsubstituted 6- $\alpha$-Man $p$ residues (such effect can be seen in the C6 signal of 6- $\alpha$ Man $p$ as detected at $\delta^{1} \mathrm{H}=3.70 / 3.87, \delta^{13} \mathrm{C}=65.26 \mathrm{ppm}$ ).

In addition, the apparent lower abundance of 2- $\alpha$-Man $p$ residues (2- $\alpha$-Man $p$ is located in the mannose caps of manLAM) may indicate a lower frequency of mannose capping or a shift in the LAM/LM ratios. The physiological outcome from any of these two possibilities is likely to be similar. As previously discussed under section 1.4.3.1, both uncapped LAM and LM are associated with the induction of a strong pro-inflammatory response, while manLAM has some immune-suppressive actions. As a result, this shift in balance towards the production of lower manLAM levels in dormant bacilli is likely to induce a corresponding change in the host immune response to the infection. Further studies are needed to confirm these findings considering the possibility that the gross morphological changes that were observed in the cells may have affected the mobility of some of the carbohydrate residues under HR-MAS acquisition conditions.

Unlike the stability observed in the mycolic acid pool under starvation conditions, some significant changes were observed in the relative abundance of mycolic acid classes. The most drastic of which was the gradual depletion of keto mycolates which completely disappeared after four weeks (based on the detection limit of this methodology). This reduction in keto mycolates levels was parallel to an increase in the levels of hydroxyl and methoxy mycolates (Figure 4.7). Ketomycolates are one of the major mycolic acid groups of $M$. bovis BCG while hydroxyl and methoxy mycolates are normally present at lower levels. It is not clear how this may affect the cell wall properties but such shift is a rational adaptation for cells that grow inside the TB granuloma as it appears that the bacterial cells may not be able to retain molecules of high oxiditative state under low oxygen conditions. 
Figure 4.6 Effect of Oxygen Depletion on the Cell Wall Polysaccharides of M. bovis BCG

HR-MAS 2D-HSQC spectra of M. bovis BCG cells grown under normal conditions, (a), grown under low oxygen conditions for four weeks, (b), and resuscitated for one day after oxygen depletion, (c). Signals circled in the three spectra are that of TAG which are usually detected at low intensity as seen in (a), but their levels reached a peak concentration after four weeks of oxygen depletion as shown in (b). Bottom inserts show the variability observed in the relative abundance of some of the LAM signals between the three spectra. Signals for 2- $\alpha-\operatorname{Man} p$ at $\delta \mathrm{H}=5.02, \delta \mathrm{C}=98.08 \mathrm{ppm}$ and that of 2,6- $\alpha-$ Man $p$ at $\delta \mathrm{H}=5.05, \delta \mathrm{C}=98.13 \mathrm{ppm}$ are circled in these inserts. In the case of cells that were grown under low oxygen for four weeks, the relative ratios of these two mannose residues changed towards the dominance of 2,6- $\alpha$-Man $p$ signals (on the left). Such change in the abundance of the 2,6 disubstituted Man $p$ residues was paralleled by a decrease in the intensity of the signals of monosubstituted 6-Manp (signal for C6 of Man $p$ are indicated by red arrows). To less extent, an increase in the number of terminal $\alpha$-Man $p$ residues can be observed as indicated by black arrows in the bottom insert. 

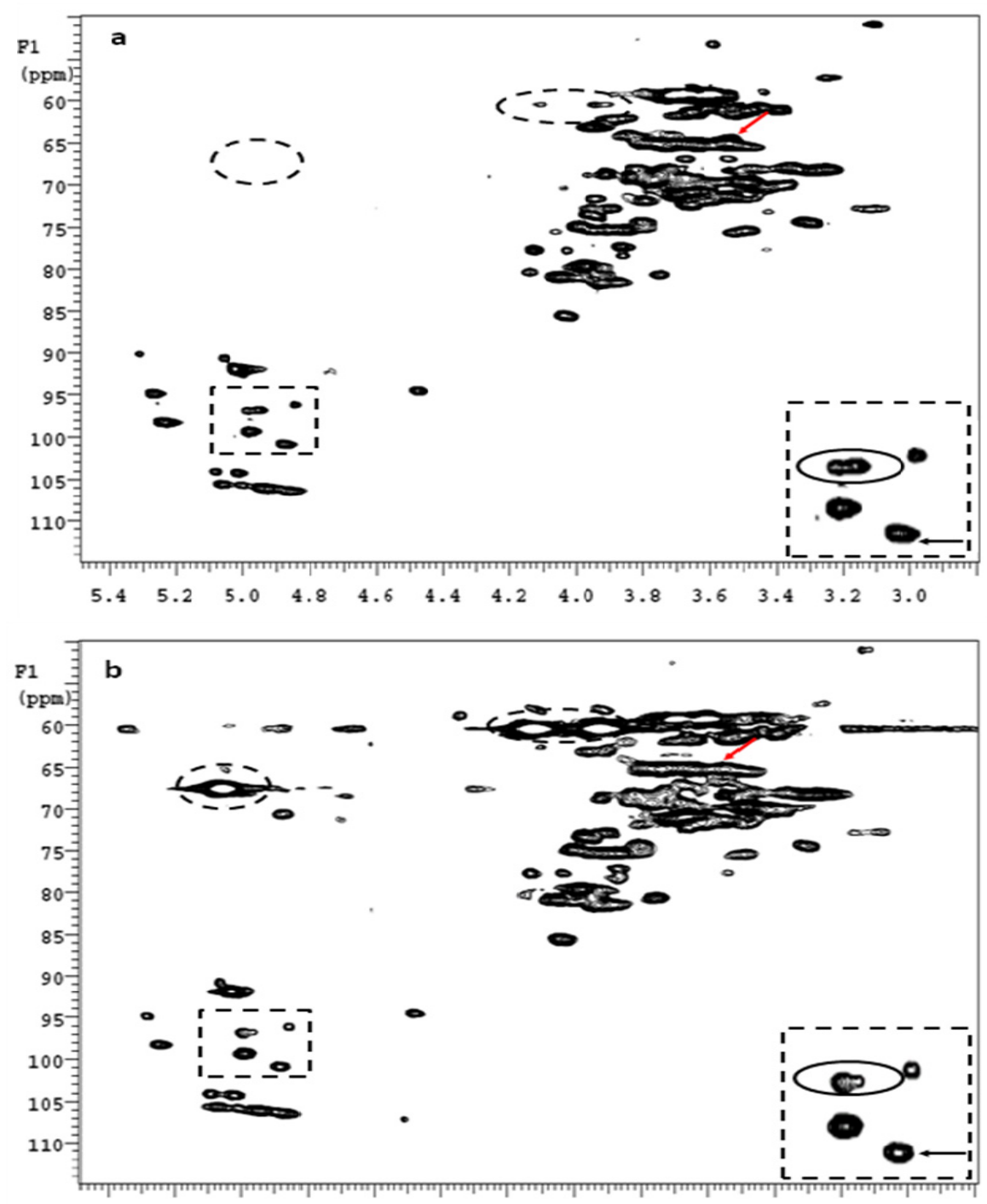

$\begin{array}{lllllllllllll}5.4 & 5.2 & 5.0 & 4.8 & 4.6 & 4.4 & 4.2 & 4.0 & 3.8 & 3.6 & 3.4 & 3.2 & 3.0\end{array}$

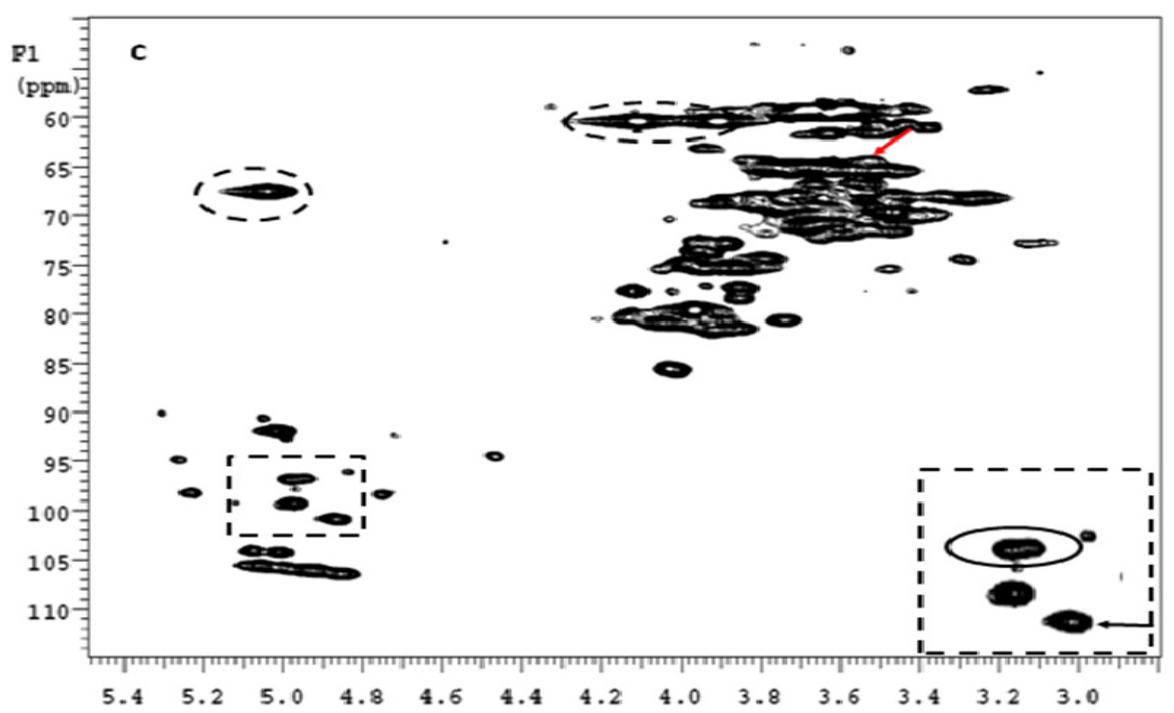




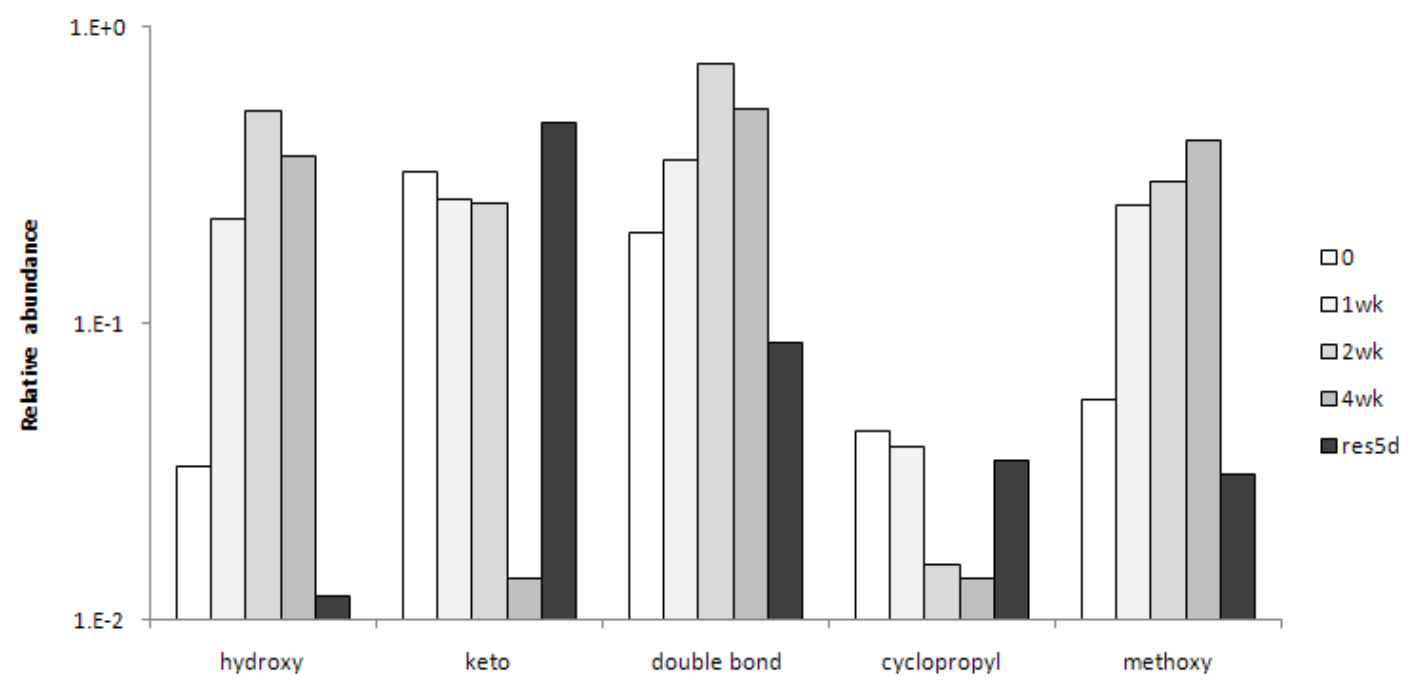

Figure 4.7 Effect of Gradual Oxygen Depletion on the Cell Wall Mycolic Acid of $M$. bovis $\mathrm{BCG}$

Relative ratios of different classes of mycolic acids are plotted as the integral volumes for their corresponding biomarker signals as described in Table 2.2 divided by the integral volume of the $\beta-\mathrm{CHOH}$ signal at $\delta \mathrm{H}=3.65, \delta \mathrm{C}=72.15 \mathrm{ppm}$. Shown here are the relative abundance of different chemical classes of mycolic acids as monitored during gradual oxygen depletion and subsequent resuscitation. Mycolic acids represented here include hydroxyl mycolate, ketomycolate, $\alpha$-unsaturated mycolates, $\alpha$-cyclopropyl mycolates and methoxy mycolates. 


\subsection{Discussion}

\subsubsection{Stability of the Structural Components of the Mycobacteria Cell Wall}

It has been well recognized that Mycobacteria have the ability to survive under harsh conditions. Such unique survival ability has led to its persistence as a human pathogen since prehistoric times. In this aspect, Mycobacteria complex and unique cell wall can be thought to act as the shield it uses to protect itself from the harsh environmental conditions it usually encounter. Whether it is inside the human macrophage or enclosed in a complex isolated granuloma, the cell wall seems to be one of the most essential components of the Mycobacteria survivosome. Hence, the integrity of the cell wall is crucial for Mycobacteria infectivity and virulence. This is most notable in the case of AG. AG structure is conserved among all Mycobacteria as discussed in the previous chapter and as we observed in this study, it also remains unaffected by different stress conditions. While these observations pinpoints to the importance of an intact AG for mycobactrial survival, it further underscore its value as an important target for the development of new drug candidates. From this study, it became obvious also that cellular levels of many other structural cell wall components were preserved despite the exposure to prolonged stress conditions to maintain an optimal cell wall integrity. In fact, most of the major changes identified in this study are related to TAG which are present for the most part inside lipid vacuoles in the cytoplasm. In case of starvation, other changes in cell wall lipid composition were found to be associated with a parallel loss of viability.

\subsubsection{Phospholipids as Indicators of Cell Viability}

From this study, we confirmed the use of phospholipids signals as indicator for cell viability. During either starvation or hypoxic conditions, the levels of PL lipids that we detected in our lipid profiles have always matched the cell viability determined by traditional microbiological techniques. This is due to the fact that upon cell death and membrane rupture, phospholipids are rapidly hydrolysed and utilized by other cells once they are not stacked in an organized lipid membrane bilayer structure. Chemical analysis of phospholipids has been used as indicator to the presence of viable bacteria in inorganic and organic samples such as water and soil in other studies. ${ }^{283,284}$ For our purpose, not only do these phospholipids serve as an indicator of cell viability, but they can be used also to ensure the quality of the lipid profiles obtained through this methodology. In comparative analysis that include different experiments, the integration value of these molecules can be used as an internal standard to which integration values of other biomarker signals can be normalized to.

\subsubsection{Triacyl Glycerols as Favorite Energy Sources}

One of the interesting results of this study was to elaborate on the role that TAG play to enhance Mycobacteria stringent response and survivability. Our results indicate that under hypoxic conditions and during exceptionally slow growth, cells have accumulated 
massive amount of TAG (up to 40 times their normal values). This result is consistent with a similar study by Daniel et al who reported overexpression of triglyceride synthases upon adaptation of the cells to hypoxic conditions. ${ }^{278}$ It can be argued that this process can be a direct result of either accumulation of TAG due to halted $\beta$-oxidation or as a measure taken by the cells in preparation for a long process of dormancy. There is more evidence however in favor of the second argument. First of all, levels of TAG started declining after reaching a peak level despite continued hypoxic conditions. Second, we also detected a short term accumulation of TAG in the first hours of transferring the cells to fully nutrient growth media in presence of normal oxygen levels. Third, we have always observed that any decline of TAG levels from previous status was matched by an increase in the levels of other organic molecules which points to the role of TAG as biosynthetic precursors. Furthermore, if we look at multicellular organisms as an assembly of unicellular ones, the behavior of hibernating animals which store their food supply with the first signs of looming winter can be extrapolated to microbial cells storing their favorite energy source after receiving signals of expected dormancy.

\subsubsection{Process of Reactivation Has Optimum Goal}

Regardless of the harsh conditions imposed on the mycobacterial cells, levels of different cellular components are always restored to a preset limit upon returning to their normal growth conditions. This observation was valid among cells that underwent partial, complete nutrient starvation or oxygen depletion. In each case however, there were certain temporary changes in the composition of cellular lipid pools especially during the first few hours of reactivation. But such temporary changes were short lived and restoration of a specific concentration to most organic molecules was achieved within two to four days and most likely as a direct result of resumed cell replication.

\subsection{Conclusion}

In conclusion, we have demonstrated the adaptive response of Mycobacteria to laboratory stress conditions that are generally accepted to mimic the in vivo environment of latent bacilli. We were able to monitor gradual changes in the cell wall composition by 2D-HSQC NMR maps using less than 100mgs of cell. This NMR-based approach allowed us to provide a comprehensive profile for different components of the cell wall lipid pool, cell wall polysaccharides and the covalently bound mycolic acid pools. As a result, we were able to detect changes in these pools and relate them to adaptive response mounted by Mycobacteria during infection. For example, we have identified the accumulation of large amount of TAG during oxygen depletion. Similar observation has been reported from bacteria isolated from diseased tissues. Electron micrographs of these bacilli indicated the presence of large lipid vacuoles that extend to cover more than $90 \%$ of the cytoplasmic space. ${ }^{7,63}$ This apparent agreement in results supports our finding and also validates the utility of in vitro oxygen depletion models. Furthermore, we observed certain changes in the LAM/LM pool of the bacilli when they were grown in the same oxygen depletion model. To the best of our knowledge, such observation has not been 
reported previously and it is a candidate for further investigation due to the shift in balance that it may impose in the Mycobacteria-host immune interaction.

This study has also validated previous reports of the unique ability of Mycobacteria to adapt to a range of harsh environmental conditions. This adaptation process is better served when the transition period to such new environment is prolonged. In our low oxygen model, we reported a very successful transition to hypoxic conditions due to an unusual slow growth rate. We also have noticed that cells that have been partially starved in low nutrient media do not suffer any measurable loss of viability upon transfer to PBS for an extended time period. Such transition periods are required for cells to adjust its metabolome for the harsh environment it expect to encounter. However, regardless of the stress conditions that the bacilli were subjected to, certain structural components (most notably AG) remained well preserved during both the stress and the recovery period. This underscores the importance of the integrity of AG for cell survivability and should encourage scientists to further study AG biosynthesis to identify new targets for anti-TB therapy beside the already established enzyme target (EMB-b) for etahmbutol. 


\section{CHAPTER 5. TWO CASE STUDIES FOR THE USE OF BIOANALYTICAL TOOLS IN SCREENING FOR VIRULENCE DETERMINANTS OF PATHOGENIC MYCOBACTERIA ${ }^{3}$}

This chapter discusses two specific examples where different spectroscopic techniques were used as a tool for targeted lipid analysis. In both cases the targeted lipid molecule is an immune-modulator. However, due to differences in their physiochemical properties, two different strategies where used for their analysis.

\subsection{Case Study-I: Screening Various Mycobacteria Species for the Production of Mycolactone Toxins}

\subsubsection{Introduction}

Mycolactones are macrolides produced by a small number of Mycobacteria species and are associated with potent immune-suppression and cytotoxic properties. ${ }^{286}$ Mycolactones were introduced to the scientific community in 1999 with the discovery of the first mycolactone from a Malaysian isolate of M. ulcerans. ${ }^{231}$ Because it was isolated as a mixture of two geometric isomers, it has been designated as mycolactone A/B. ${ }^{287}$ This was the first report of macrolide production by a mycobacterium although macrolides in general have been isolated from many other organisms most notably Streptomyces species. ${ }^{239}$ Other analogues of mycolactone A/B were later detected in different M. ulcerans isolates and other closely related Mycobacteria. ${ }^{254,285,288-290}$ All mycolactones share a totally conserved common macrolide core (a 12-membered lactone ring conserved in its chemical substitution and stereochemistry). This core is esterfied at C-5 position to a polyene acidic side chain. Mycolactone molecules identified that far show only variability in the length and substitution of their side chains (Figure 5.1 and Table 5.1).

All Mycobacteria producing mycolactone (MPM) contain a large plasmid that encodes three large polyketide synthase (PKS) genes responsible for mycolactone biosynthesis $(m l s A 1, m l s A 2$ and $m l s B) .{ }^{291}$ All three genes belong to the bacterial PKS-type I class where the PKS consist of several multi-enzyme modules that work together as a molecular assembly line. Every module consists of at least three basic proteins; ketosynthase (KS), Acyl transferase (AT) and acyl carrier protein (ACP) and is responsible for addition of a specific unit to the growing molecule (Figure 5.2) ${ }^{291}$ More diversity among these building units results from the activity of additional enzymes in specific modules. Such enzymes include keto reductase (KR), dehydratase (DH) and enoyl reductase (ER). ${ }^{292}$

\footnotetext{
${ }^{3}$ Parts of this chapter are adapted with permission. Ranger, B.S. et al. Globally distributed mycobacterial fish pathogens produce a novel plasmid-encoded toxic macrolide, mycolactone F. Infect Immun 74, 6037-45 (2006). ${ }^{285}$
} 


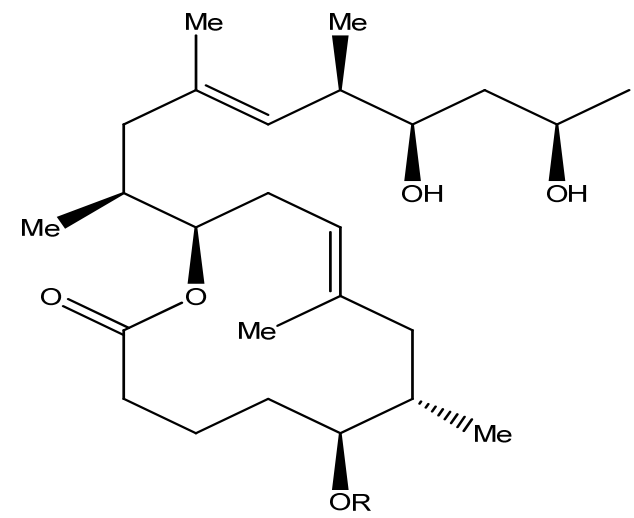

Figure 5.1 General Structure of Mycolactones

Table 5.1 Differences in the Structures of Mycolactones Isolated So Far

Side Chain (R) $\quad$ Name $\begin{aligned} & \begin{array}{l}\text { Parent Strain (Host) } \\ \text { Location of Isolation }\end{array} \\ & \text { Mwt References }\end{aligned}$




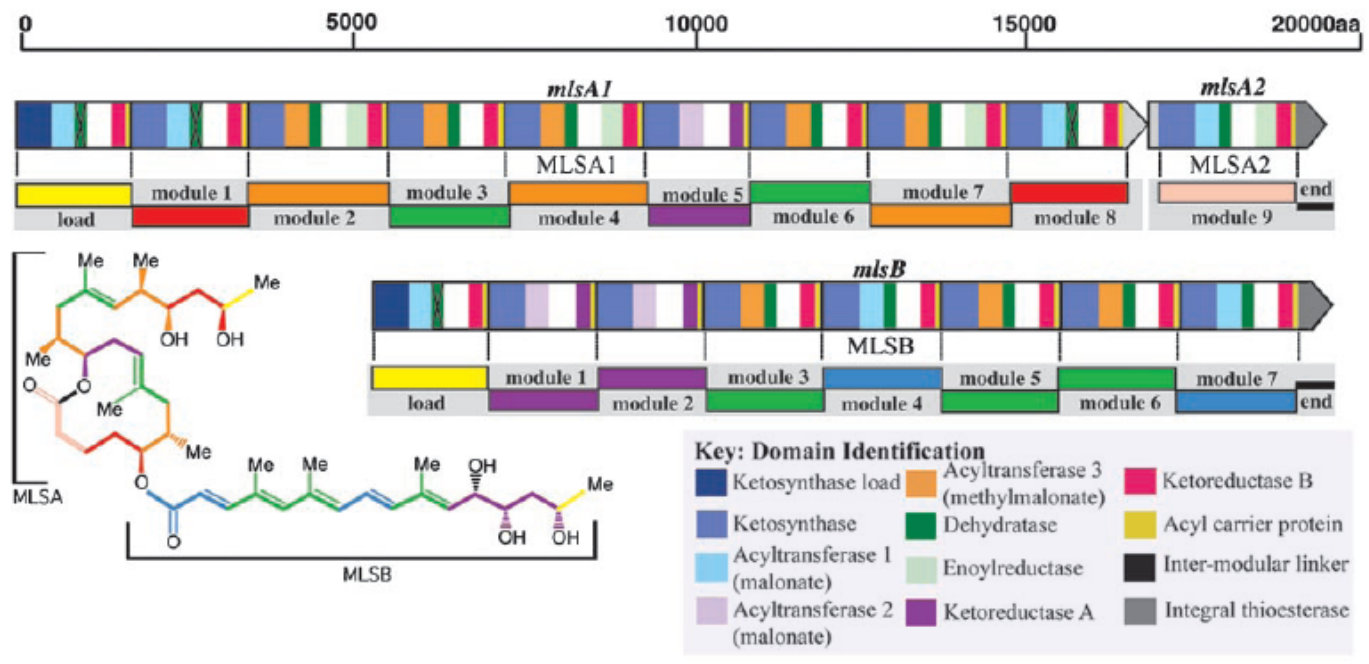

Figure 5.2 Domain and Module Organization of Mycolactone A/B PKS Genes

Within each of the three genes ( $m l s A 1, m l_{s} A 2$ and $\left.m l_{s} B\right)$, different domains are represented by a colored block. The domain designation is described in the key. White blocks represent interdomain regions of $100 \%$ identity. The structure of mycolactone has also been color-coded to match the module responsible for a particular chain extension. Reprinted with permission. Stinear, T.P. et al. Giant plasmid-encoded polyketide synthases produce the macrolide toxin of Mycobacterium ulcerans. Proc Natl Acad Sci USA 101, 1345-9 (2004). ${ }^{291}$ 
Similarly, structural variations between mycolactones produced by different MPM are attributed to genetic variability in specific modules which translates to addition or elimination of a specific structural feature in the growing chain. ${ }^{286,291,292}$

The first mycolactone, isolated from M. ulcerans 1615 strain, was found to be a 2:3 mixture of two double bond isomers hence was the designation mycolactone $\mathrm{A} / \mathrm{B} \cdot{ }^{287}$ Most other mycolactones exist in the form of an equilibrating mixture of two major geometric isomers. This isomerization is a result from unrestricted rotation around 4' -5 ' double bond. For example, mycolactone $\mathrm{A} / \mathrm{B}$ is a $2: 3$ mixture of $\mathrm{Z}-\Delta^{4,}, 5^{\prime}$ and $\mathrm{E}-\Delta^{4,}, 5^{\prime}$ isomers and mycloctone $\mathrm{C}$ is a $1: 1$ mixture of two similar Z- $\Delta^{4,}, 5^{\prime}$ and $\mathrm{E}-\Delta^{4,}, 5^{\prime}$ isomers. ${ }^{295}$

Due to their structure, mycolactone are readily ionizable molecules that can be easily detected by MS as sodium adducts at $[\mathrm{Mwt}+23]$ in the positive mode. ${ }^{290}$ On the other hand, the chemical instability of the polyene structure and the possible isomerization due to photo-oxidation can result in a mixture of a large number of geometric isomers especially in aged samples. This large number of possible isomers can result in multiple overlapping signals during NMR acquisition and mar the accurate interpretation of the NMR data. Also the relatively low abundance of these molecules $(1 \mathrm{mg} /$ liter in culture grown in T-flask $)^{299}$ makes MS better suited for their accurate and efficient analysis.

\subsubsection{Materials and Methods}

\subsubsection{Bacterial culture and growth conditions}

All isolates were grown either on plates of Middlebrook 7H11 agar media supplemented with $10 \%$ oleic acid-ADG supplement, or in T-flasks in Middlebrook 7H9 supplemented with $10 \%$ ADG and $0.05 \%$ Tween 80 . M. ulcerans strains were incubated at $32^{\circ} \mathrm{C}$. All other isolates were grown at room temperature.

\subsubsection{Lipid extraction}

Lipids were extracted from M. ulcerans using chloroform:methanol 2:1 (vol/vol) and phospholipids were removed by precipitation with ice cold acetone to obtain acetonesoluble lipids (ASLs). Purified mycolactone was obtained using centripetal silica chromatography with a chromatotron after elution with chloroform:methanol:water 90:10:1 ( $\mathrm{vol} / \mathrm{vol} / \mathrm{vol})$.

\subsubsection{HPLC analysis}

Methanolic extracts of ASLs were analyzed using Shimadzu LC-20AD analytical HPLC with UV detection at $360 \mathrm{~nm}$. Separation of various mycolactones was obtained on a Phenomenex-Luna, C18 250X4.6 $\mathrm{mm}$ column at $2 \mathrm{ml} / \mathrm{min}$ flow rate with water/acetonitrile gradient: $55 \%$ acetonitrile $3 \mathrm{~min}$; isocratic followed by a 37 min linear gradient to $100 \%$ acetonitrile. 


\subsubsection{MS analysis}

UV active fractions were analyzed offline on an ion trap ESI Bruker-Esquire mass spectrometer. MS analysis conditions: dry temperature of $300^{\circ} \mathrm{C}$, gas flow of $5 \mathrm{~L} / \mathrm{min}$ and nebulizer pressure of 15 psi. For preliminary screening, the methanolic extracts of ASLs were directly analyzed without chromatographic separation.

\subsubsection{NMR analysis}

Two milligrams of mycolactone F, purified from M. marinum DL240490 strain, was dissolved in acetone- $\mathrm{d}_{6}(500 \mu \mathrm{l}) .{ }^{1} \mathrm{H}$ NMR and 2D-COSY spectra were recorded on a Varian Inova spectrometer at $500 \mathrm{MHz}$. Chemical shifts were reported in ppm relative to the residual acetone peak at $2.09 \mathrm{ppm}$.

\subsubsection{Results}

\subsubsection{Screening by MS/MS and detection of mycolactones}

Previously identified mycolactones and co-metabolites share a conserved core structure with variation only in the substitution of the side chain. It is very advantageous for MS analysis that the fragmentation of mycolactone often results in the formation of a lactone core fragment through McLafferty rearrangement (Figure 5.3). This fragment, detected at $\mathrm{m} / \mathrm{z}=429$, is indicative of the conserved mycolactone core sodium adduct. This fact can be used to identify mycolactones directly from cellular lipid extract by screening for ions that can produce such fragment. In the case of Mycolactone $\mathrm{A} / \mathrm{B}$, upon capture and fragmentation of the precursor mycolactone adduct, it produces two main ions; that of the core at $\mathrm{m} / \mathrm{z}=429$ and the other for the side chain at $\mathrm{m} / \mathrm{z}=359$ (Figure 5.3).

Therefore, MS analysis of ASLs can be a very useful tool in screening for new mycolactones. ${ }^{289,290} \mathrm{MS}$ analysis of several M. ulcerans isolates from Africa showed precursor ion and fragment peaks consistent with that of mycolactone A/B. However, different molecular ion peaks were observed in isolates from China where the dominant ions where detected at $\mathrm{m} / \mathrm{z}=779$ and 777 . Upon fragmentation, these precursor ions produced the core fragment at $\mathrm{m} / \mathrm{z}=429.4$ and the side chain fragment at $\mathrm{m} / \mathrm{z}=373$ and 371 respectively. This pattern is in agreement with the presence of Mycolactone D. ${ }^{296}$

M. ulcerans 842 isolate from South America produced two major mycolactone ions at $\mathrm{m} / \mathrm{z}=763$ and 737 . The later, now identified as mycolactone $E$, produced a side chain fragment ion at $\mathrm{m} / \mathrm{z}=331$ while the precursor ion at $\mathrm{m} / \mathrm{z}=763$ produces a product ion at $\mathrm{m} / \mathrm{z}=357$. Similar patterns were observed upon the analysis of ASLs from different $M$. liflandii isolates from two laboratory colonies of Xenopus tropicalis and Xenopus lavis. However, no peaks at $\mathrm{m} / 763$ were observed. This indicates that while both the South America M. ulcerans isolates and the frog pathogen M. liflandii produce similar molecule (mycolactone E), there seems to be a limit to the length of the side chain of mycolactones produced by the frog pathogen. 


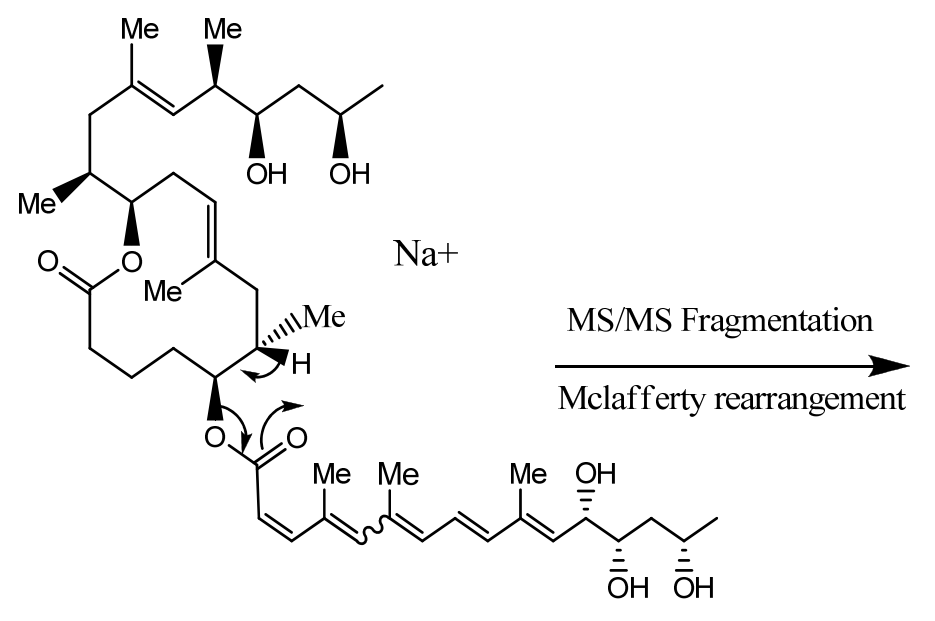

Mycolactone A/B

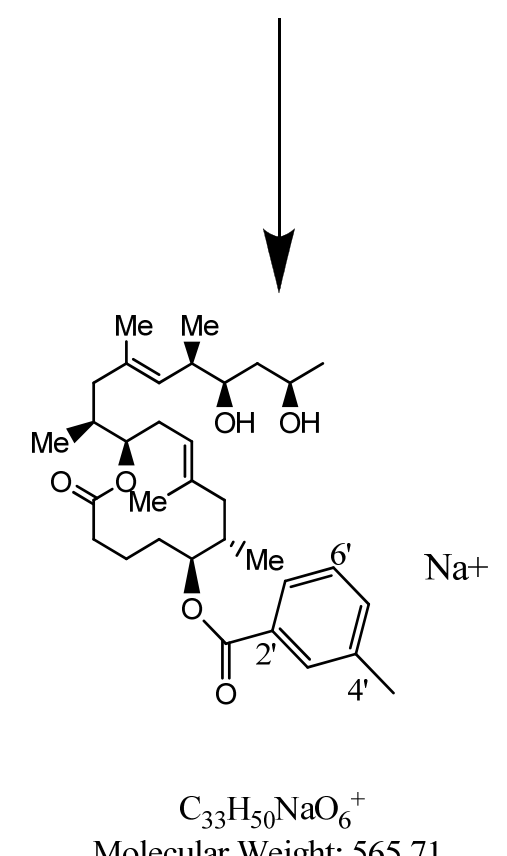

Molecular Weight: 565.71

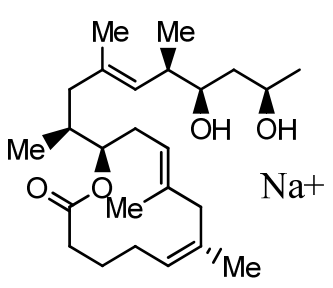

Mycolactone core

$\mathrm{C}_{25} \mathrm{H}_{44} \mathrm{O}_{4} \mathrm{Na}^{+}$

$\mathrm{m} / \mathrm{z}=429.324$

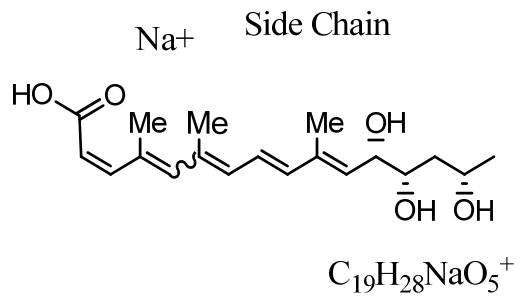

Molecular Weight: 359.41

Figure 5.3 Major Fragmentation Pathways of Mycolactone A/B

MS fragmentation of mycolactone A/B proceed through Mclafferty rearrangement to produce two characteristic product ions; that of the core at $\mathrm{m} / \mathrm{z}=429$ and the side chain at $\mathrm{m} / \mathrm{z}=359$. All mycolactone molecules produce the same core fragment while their side chain fragments differ in their $\mathrm{m} / \mathrm{z}$ based on the side chain substitution. Another fragmentation pathway produces a product ion at $\mathrm{m} / \mathrm{z}=565.7$ is common among all mycolactone that have the same side chain substitution between $\mathrm{C} 1$ ' $-\mathrm{C}-6$ ' ${ }^{288,296}$ 
A newly identified organism (temporarily identified as M. shinshuense. and currently as M. ulcerans subsp. shinshuense) was isolated in Japan from a small number of patients with cases of skin lesions similar to that of Buruli ulcer. ${ }^{300,301}$ Direct MS analysis of the ASL extract from this isolate identified at least three major mycolactones at $\mathrm{m} / \mathrm{z}=793$, 779 and 777. All three ions where associated with a product ion of the core lactone ring upon fragmentation (Figure 5.4a,b). Their side chain product ions where detected as expected at $\mathrm{m} / \mathrm{z}=387,373$ and 371 respectively. A Peak indicative of mycolactone A/B at $\mathrm{m} / \mathrm{z}=765$ was detected as well.

Finally, MS analysis for the ASL of M. pseudoshottsii L15 (a pathogen isolated from sea striped bass found in the Chesapeake Bay) ${ }^{42}$ and nine isolates of M. marinum isolated from infected fish in the Middle East region revealed a major ion at $\mathrm{m} / \mathrm{z}=723[\mathrm{M}+\mathrm{Na}]^{+}$. Upon fragmentation, this precursor ion produced the conserved core fragment ion at $\mathrm{m} / \mathrm{z}=429$ suggesting the presence of another mycolactone, later designated as mycolactone F (Figure 5.4c,d). Moreover, the fragmentation patterns in all samples were identical suggesting that the mycolactones produced by these strains have the same chemical structure. In all ten cases, the side chain fragment was detected at $m / z=317$. Other mycolactone characteristic product ions where detected at $\mathrm{m} / \mathrm{z}=635,565$ and 273. The complete results of MS screening of the various lipid extract are summarized in Table 5.2.

\subsubsection{HPLC profiles of different mycolactones}

Because of the presence of six conjugated double bond in the side chain of mycolactone $\mathrm{A} / \mathrm{B}$, it is relatively easy for mycolactone to undergo double bond isomerization. It is well known that 1,3,5-hexatriene can undergo double bond isomerization at $\mathrm{C} 3-\mathrm{C} 4$ through a biradical transition state as a result of photo-oxidation. Similarly, due to the extended conjugation in the mycolactone side chain, unrestricted rotation is possible around $\mathrm{C}^{\prime}$ '- $\mathrm{C} 5$ ' double bond. This free rotation is responsible for the existence of mycolactones as equilibrating mixture of double bond isomers.

Traditional HPLC columns are capable of separating these geometric isomers. But once outside the column, each isomer is converted to an equilibrating isomeric mixture of specific ratio. For further structural analysis, it is necessary to understand the nature of each mycolactone as a mixture of isomers rather than a single molecule. Under the condition described in section 5.1.2.3, mycolactone A/B was separated by HPLC to two major isomers with comparable concentration at 20.1 and 23.8 minutes (Figure 5.5a). Other isomers of the same molecular weight were also detected. In the case of mycolactone $\mathrm{E}$ and $\mathrm{F}$, the side chain lacks one of the double bonds of mycolactone $\mathrm{A} / \mathrm{B}$. This results in apparent stability of these two molecules. At room temperature, a dominant isomer is detected by HPLC at 23.2 minutes in case of mycolactone $\mathrm{E}$ and at $20.5 \mathrm{~min}$ in case of mycolactone $\mathrm{F}$ (Figure 5.5d). The production of the same molecule (mycolactone F) by multiple strains was also confirmed through the production of similar HPLC chromatogram by ten of the strains tested in this study. 

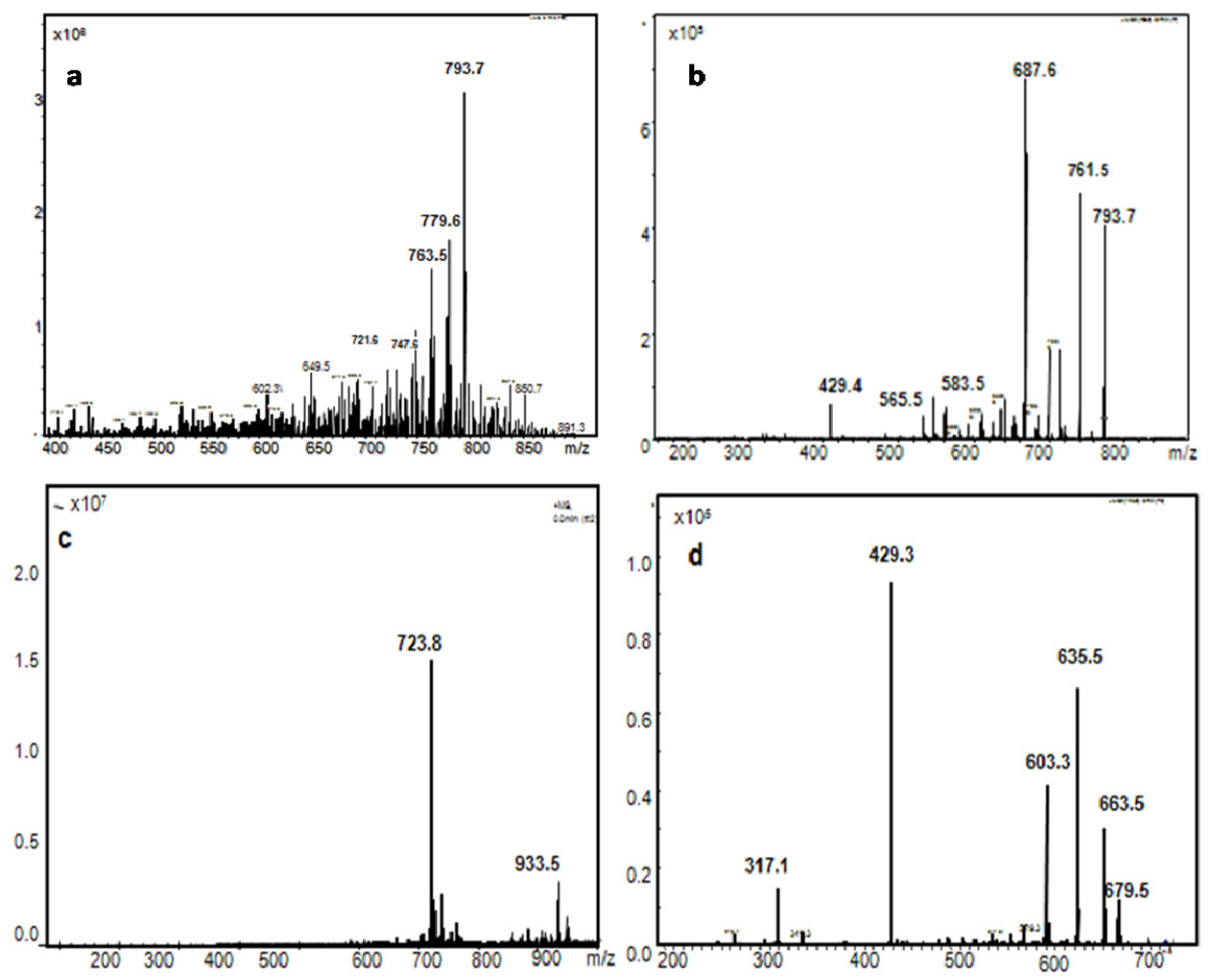

Figure 5.4 MS Analysis of Different Mycolactone Molecules

MS analysis of the ASL of M. ulcerans subsp. shinshuense showing three major mycolactones at $\mathrm{m} / \mathrm{z}=793,779$ and 763 , (a). Fragmentation of the $\mathrm{m} / \mathrm{z}=793$ precursor ion result in the production of the mycolactone core ion at $\mathrm{m} / \mathrm{z}=429.4$, (b). MS analysis of the ASL of M. pseudoshottsii L15 showing the dominance of a major mycolactone ion at $\mathrm{m} / \mathrm{z}=723$, (c). Fragmentation of the precursor ion at $\mathrm{m} / \mathrm{z}=723$ produces a core ring product ion at $\mathrm{m} / \mathrm{z}=429$ and a side chain product ion at $\mathrm{m} / \mathrm{z}=317$, (d). MS analysis conditions were described under section 5.1.2.4. 
Table 5.2 Mycolactone Ions Identified in Different Mycobacteria Strains

\begin{tabular}{|c|c|c|}
\hline Strain & Origin & {$\left[\mathrm{M}+\mathrm{Na}^{+}\right]$Ions } \\
\hline M. ulcerans 1615 & Human Malaysia & $\mathbf{7 6 5}, 747,749$ \\
\hline M. ulcerans 98-912 & Human, China & 779, 777 \\
\hline M. ulcerans 842 & Human, S. America & $\mathbf{7 6 3}, 761,737$ \\
\hline M. ulcerans subsp shinshuense & Human, Japan & 793, 779, 777 \\
\hline M. liflandii 128 & Frog, ,USA & 737,735 \\
\hline M. liflandii HW-1 & Frog, USA & $\mathbf{7 3 7}, 751,733$ \\
\hline M. liflandii XL-5 & Frog, USA & $\mathbf{7 3 7}, 753,735$ \\
\hline M. pseudoshottsii L15 & Fish, Chesapeake Bay & $\mathbf{7 2 3}, 763,755,739$ \\
\hline M. marinum DL240490 & Fish, Red Sea & $\mathbf{7 2 3}, 763,755,739$ \\
\hline M. marinum SA200695 & Fish, Red Sea & $\mathbf{7 2 3}, 755,739$ \\
\hline M. marinum Hyb270995 & Fish, Red Sea & $\mathbf{7 2 3}, 757,739$ \\
\hline M. marinum DL150991 & Fish, Red Sea & $\mathbf{7 2 3}, 757,755,739$ \\
\hline M. marinum DL180 & Fish, Red sea & $\mathbf{7 2 3}, 755,739$ \\
\hline M. marinum CFO30494 & Fish, Red Sea & $\mathbf{7 2 3}, .757, .755, .739$ \\
\hline M. marinum DL045 & Fish, Red sea & 723, 739 \\
\hline M. marinum CC240299 & Fish, Red Sea & $\mathbf{7 2 3}, 739,721$ \\
\hline M. marinum BB170200 & Fish, Red Sea & $\mathbf{7 2 3}, 755, .739$ \\
\hline
\end{tabular}

Major mycolactone ions are highlighted in bold numbers. 

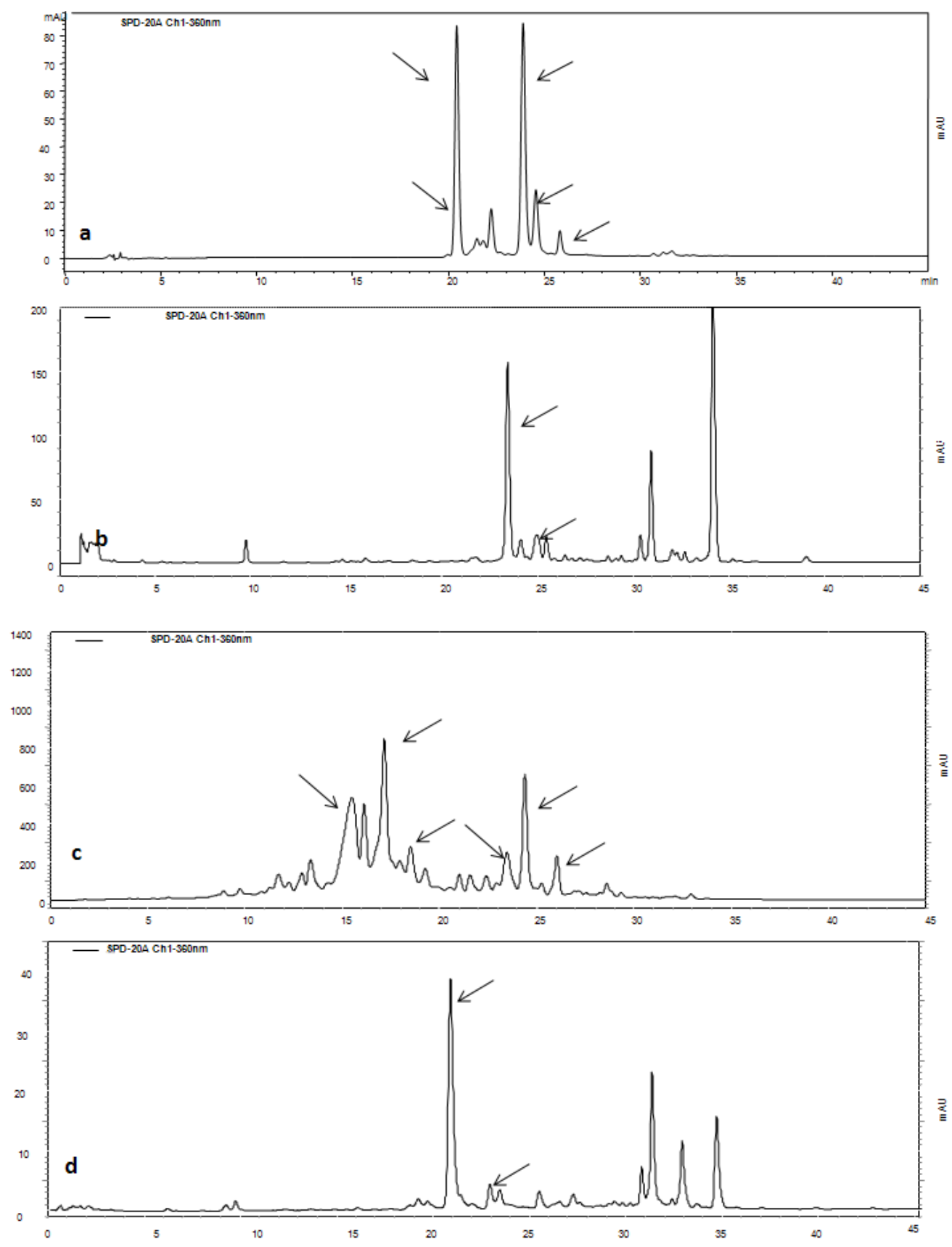

Figure 5.5 HPLC Profiles of the ASL Extract from Different Mycobacteria

HPLC chromatogram of the ASL obtained from M. ulcerans 1615, (a). Arrows indicate the elution of mycolactone $\mathrm{A} / \mathrm{B}$ isomers with $\mathrm{m} / \mathrm{z}=765$. HPLC profile of ASL from $M$. liflandii XL-5, (b). Arrows indicate the elution of a mycolactone $\mathrm{E}$ isomer at $\mathrm{m} / \mathrm{z}=737$. HPLC profile of ASL from M. ulcerans subsp. shinshuense, (c). Arrows indicate the elution of different mycolactone molecules of variable $\mathrm{m} / \mathrm{z}$. HPLC chromatogram of the ASL of M. marinum DL240490, (d). Arrows indicate the elution of mycolactone molecules $\mathrm{m} / \mathrm{z}=723$. HPLC and MS analysis conditions were describes in sections 5.1.2.3 and 5.1.2.4 respectively. 
Finally, The HPLC chromatogram of the ASL extract from M. ulcerans subsp. shinshuense showed more complexity than all previous cases (Figure 5.5c). In part, this is due to the presence of at least three mycolactone analogs as detected by MS analysis but double bond isomerization have significant contribution to this complexity as inferred from offline MS analysis of the HPLC eluted peaks.

\subsubsection{Structural determination of mycolactone $F$}

Although mass spectrometry is an increasingly valuable tool for molecular analysis, data from NMR analysis provides a more rigorous method for structural assignments. The limited amount of mycolactone produced by mycobacterial species as well as the very slow growth rate of MPM has made it extremely difficult to obtain sufficient mycolactone for optimal structural analysis. However, the relatively robust growth yield of lipids from some M. marinum isolates made it possible to undertake a more thorough analysis of mycolactone F structure isolated from M. marinum DL240490 strain.

To determine the structure of mycolactone $\mathrm{F}, 1 \mathrm{D}$ and 2D NMR experiments were performed on purified sample of mycolactone F. As expected, ${ }^{1} \mathrm{H}$ NMR revealed a structure very similar to that reported for mycolactones $\mathrm{A} / \mathrm{B}$ with the characteristic core proton signals at C9, C15, C11 and C5 easily identified at 5.16, 5.03, 4.93 and $4.75 \mathrm{ppm}$ respectively ${ }^{287}$ (Figure 5.6). In the region corresponding to the side chain, two geometrical isomers were distinguished with an integration ratio of 10:1. Both isomers showed five olefinic protons. For the major isomer, the most downfield proton was that of C3' which appeared as a doublet at $7.39 \mathrm{ppm}$ and was coupled to $\mathrm{C} 2$ ' proton (doublet at $5.91 \mathrm{ppm}$ ). The presence of two singlet peaks at 6.4 and $6.1 \mathrm{ppm}$ suggested the presence of methyl substitutions at C4', C6' and C8'. This was confirmed by 2D-COSY analysis which showed the long range coupling of these olefinic protons to methyl groups at 2.06 and $2.03 \mathrm{ppm}$. The remaining side chain signals from $\mathrm{C} 10^{\prime}$ to $\mathrm{C} 15^{\prime}$ ' overlapped signals from the mycolactone core in the $1 \mathrm{D}-{ }^{1} \mathrm{H}$ NMR. Thus, $2 \mathrm{D}-\mathrm{COSY}$ was used to assign these protons (C9' to $\mathrm{C} 14^{\prime}$ ) using the off diagonal ${ }^{1} \mathrm{H}-{ }^{1} \mathrm{H}$ correlation peaks (Table 5.3). Mycolactone $F$ is the smallest mycolactone identified so far and as with other mycolactone variants, the core lactone structure is identical to that of other mycolactones, whereas the side chain is unique.

\subsubsection{Discussion}

The use of MS for the structural analysis and screening for mycolactones is a very efficient approach. Unlike most cellular lipids that posses long hydrophobic fatty acid chain, mycolactones are polyketide in nature and their substitution pattern contain many oxygenated function that make their ionization by soft techniques like ESI more efficient. It is also useful that variations in the mycolactone structures are restricted to the side chain and that the fragmentation pattern of mycolactone follows simple and often predictable pathways. However, for a complete structural assignment, NMR provides the necessary tool for structural determination provided that the analyzed sample is of enough purity and shows significant chemical stability. 


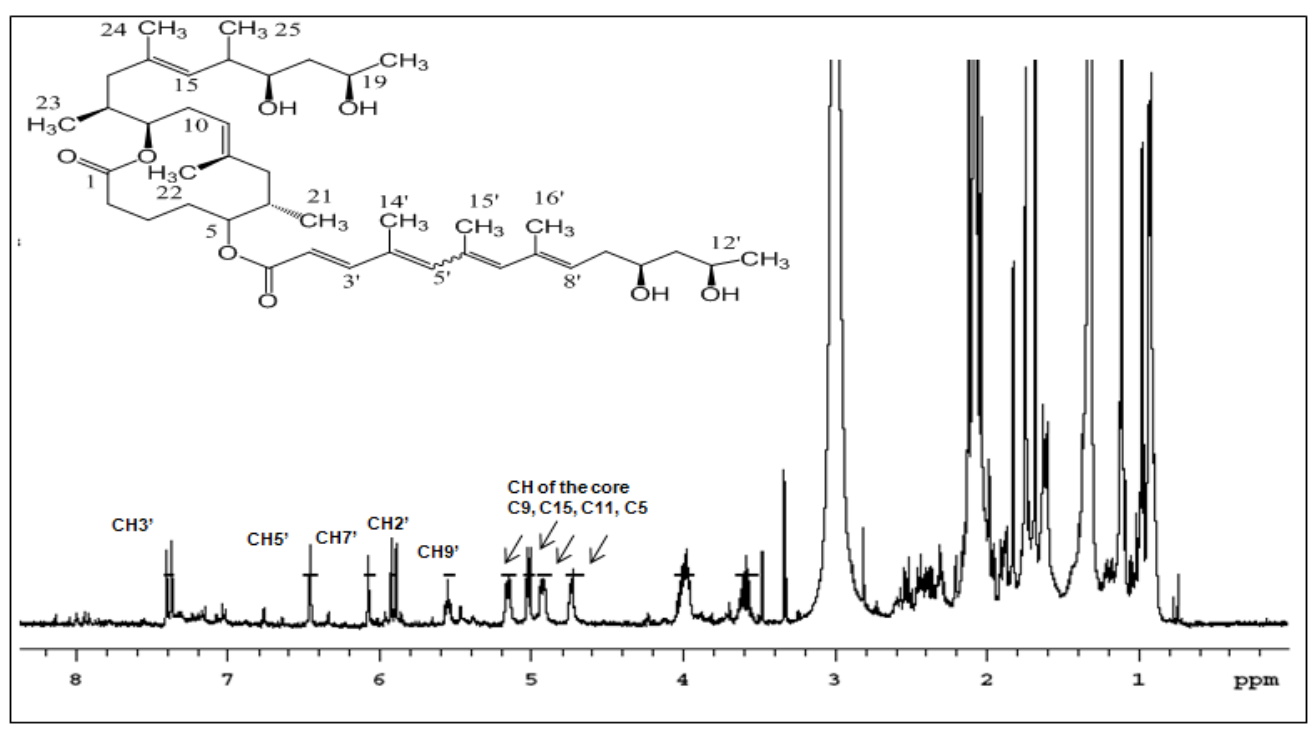

Figure 5.6 Structure and ${ }^{1} \mathrm{H}-\mathrm{NMR}$ of Mycolactone F

Table 5.3 Proton Chemical Shifts of the Major Isomer of Mycolactone F

\begin{tabular}{cccccccc}
\hline \multicolumn{2}{c}{ Side Chain } & \multicolumn{2}{c}{ Core Ring } & \multicolumn{2}{c}{ Side Chain } & \multicolumn{2}{c}{ Core Ring } \\
\hline Atom & $\delta^{1} \mathrm{H}(\mathrm{ppm})$ & Atom & $\delta^{1} \mathrm{H}(\mathrm{ppm})$ & Atom & $\delta^{1} \mathrm{H}(\mathrm{ppm})$ & Atom & $\delta^{1} \mathrm{H}(\mathrm{ppm})$ \\
\hline $2^{\prime}$ & $5.91(\mathrm{~d})$ & 2 & $2.45,2.04$ & $16^{\prime}$ & 2.03 & 15 & 5.03 \\
$3^{\prime}$ & $7.39(\mathrm{~d})$ & 3 & 1.60 & $17^{\prime}$ & 2.06 & 16 & 2.39 \\
$5^{\prime}$ & $6.46(\mathrm{~s})$ & 4 & 1.7 & & & 17 & 3.5 \\
$7^{\prime}$ & $6.09(\mathrm{~s})$ & 5 & 4.75 & & & 18 & $1.62,1.13$ \\
$9^{\prime}$ & $5.56(\mathrm{t})$ & 6 & 2.01 & & & 19 & 3.99 \\
$10^{\prime}$ & $2.31(\mathrm{~m})$ & 7 & 1.92 & & & 20 & 1.13 \\
$11^{\prime}$ & 3.99 & 9 & 5.16 & & & 21 & 1.00 \\
$12^{\prime}$ & 1.12 & 10 & $2.51,2.12$ & & & 22 & 1.7 \\
$13^{\prime}$ & 3.56 & 11 & 4.93 & & & 23 & 1.03 \\
$14^{\prime}$ & 1.03 & 12 & 2.09 & & & 24 & 1.69 \\
$15^{\prime}$ & 1.06 & 13 & 2.14 & & & \\
\hline
\end{tabular}


It was possible to determine that all ten fish pathogen tested here produced the same molecule through analysis of both MS/MS and HPLC analysis. The production of the same product ions upon fragmentation of the precursor ion at $\mathrm{m} / \mathrm{z}=723$ indicated that the side chain of all these molecules are the same. On the other hand, consistency in the HPLC profiles of the equilibrating double bond mixtures further confirmed this observation. However in both cases, differences in the stereochemistry between these mycolactone F molecules can not be ruled out. Recently, the structure of mycolactone F was confirmed through chemical synthesis and its absolute stereochemistry has been assigned. $^{302}$

The structure of mycolactone D has been proposed by Hong et al through a detailed examination of all the product ions it produced upon its fragmentation in MS/MS mode. ${ }^{296}$ The presence of a C2' methyl in mycolactone $\mathrm{D}(\mathrm{m} / \mathrm{z}=779)$ was suggested based on the apparent mass increase in one of the mycolactone $A / B$ fragment from $\mathrm{m} / \mathrm{z}=565.4$ to 579.6. This ion represents the fragmentation product due to aromatization of the side chain at $\mathrm{C} 1$ '-C6'. Due to the mass increase in this ion by 14 dalton in mycolactone $\mathrm{D}$, the presence of a methyl group was suggested and its only possible location was C2', ${ }^{296}$

The utilization of the same approach with the mycolactones identified in the ASL of M. ulcerans subsp shinshuense come to the conclusion that the three mycolactones in this extract detected at $\mathrm{m} / \mathrm{z}$ of 777,779 and 793 are not structurally similar to mycolactone D and are more likely to be related to mycolactone A/B. The presence of C2' methyl can be immediately ruled out on the basis of the production of the $\mathrm{m} / \mathrm{z}=565.4$ fragment. Also ${ }^{1} \mathrm{H}$ NMR and $2 \mathrm{D}-{ }^{1} \mathrm{H}-{ }^{1} \mathrm{H}$ COSY analysis of a mixture of these three molecules indicated the presence of three sets of coupled protons at $\delta^{1} \mathrm{H}=\left(2^{\prime} \mathrm{CH}\right.$ at $5.96,3^{\prime} \mathrm{CH}$ at $\left.7.96 \mathrm{ppm}\right)$, ( $2^{\prime} \mathrm{CH}$ at $5.88,3^{\prime} \mathrm{CH}$ at $\left.7.48 \mathrm{ppm}\right)$ and $\left(2^{\prime} \mathrm{CH}\right.$ at $5.98,3^{\prime} \mathrm{CH}$ at $\left.7.52 \mathrm{ppm}\right)$. No other offdiagonal signals were detected between protons in the olefeinc region which may indicate the presence of methyl substitution at C8'. Unfortunately, due to the existence of multiple isomers in that mixture (as indicated from its HPLC chromatogram), further structural characterization was not possible at the time.

From our screening of MPM isolates, we observed that all M. ulcerans isolates (including subspecies shinshuense) produce mycolactones where the side chain reaches 16 carbons in length. This length is consistent with a PKS-I enzyme with seven modules in addition to the loading domain. Differences in the side chain between M. ulcerans mycolactones were restricted two areas. First, the substrate of one or more module can change from malonate to methylmalonate resulting in an additional methyl branch (as in mycolactone D). Second, the C12' hydroxyl which is added by a p450 monoxygenase may be absent (as in the case of mycolactone C). While M. ulcerans isolated from South America produces a mycolactone with a shorter side chain, mycolactoneE, longer chain analogues were also observed at $\mathrm{m} / \mathrm{z}=763$ and 761 . This confirms the presence of a PKS-I with seven modules. In mycolactones produced by both frog and fish pathogens, the side chain length was $\mathrm{C} 15$ and $\mathrm{C} 14$ respectively. This suggests the presence of PKS-I of only six active modules in addition to the loading domain. Differences between mycolactone $\mathrm{E}$, produced by the frog pathogen, and mycolactone $\mathrm{F}$, produced by fish pathogens, were limited to the loading residue which is either methylmalonyl residue in the first or 
malonyl in the latter. Some of these findings have been supported recently through an elegant genomic study by Pidot, S et $a 1^{292}$

These structural variations between different mycolactones are translated to different cytopathic activities of mycolactones in standard in vitro assays. Mycolactone $A / B$ is known to cause $\mathrm{G}_{0} / \mathrm{G}_{1}$ cycle arrest and produce cytopathic activity (CPA) at concentration less than $1 \mathrm{ng} / \mathrm{ml} .{ }^{231}$ Mycolactone $\mathrm{C}$ which has the same chain length but lacks the hydroxyl group at C12' demonstrates comparable CPA but at much higher concentration $(800 \mathrm{ng} / \mathrm{ml}) .{ }^{290}$ Shorter side chains (as in mycolactone E and F) are also associated with reduction in CPA. For example, mycolactone E (C15 side chain) produces CPA at concentration of $100 \mathrm{ng} / \mathrm{ml} .{ }^{254}$ With regards to their biosynthesis and the range of biological activities attributed to them, mycolactones are fascinating bacterial metabolites. Understanding their "structure activity relationship" as well as the genetic bases behind it should encourage researchers to develop combinatorial biosynthetic strategies targeted at the production of mycolactone analogs. Such effort will help further elucidate the effect of substitution on mycolactone biological activity and might produce novel drug candidates that can be used in the treatment of cancer or auto immune diseases.

\subsection{Case Study II: Screening Mycobacterium tuberculosis Clinical Isolates for the Production of PGL}

\subsubsection{Introduction}

Phenolic glycolipids (PGL) have been previously isolated from M. leprae, $M$. kansasii, M. marinum, M. bovis and M. tuberculosis Canetti strain, a smooth colony variant of $M$. tuberculosis. ${ }^{144,145,260,262,303,304}$ In a previous chapter, we have reported the isolation of PGL from M. liflandii as well (section 3.3.4). The aglycon part of PGL is usually phenolphthiocerol-A, phenolphthiotriol-A or phenolphthiodiolone esterified to two molecules of mycocerosic acid. The glycon part which substitutes the oxygen of the phenol ring can be either monosaccharide, disaccharide or trisaccharide. ${ }^{149,262,303}$ Examples of mycobacterial PGL were previously shown in Figure 1.11.

The aglycon part of PGL can be considered as an aromatic derivative of PDIM (section 1.4.3.6). Different mutants of M. tuberculosis that are deficient in the biosynthesis of PDIM have been shown to be attenuated in a murine model by different research groups. ${ }^{146,305}$ However, the specific role of PDIM in virulence has not been fully elucidated. It has also been suggested that the apparent attenuation of PDIM deficient mutant is due mainly to gross structural modification of the cell wall. ${ }^{306}$

PGLs are associated with significant immune-modulatory effects that are well supported by plethora of evidence. PGL-I of M. leprae is the main antigenic component of this pathogen ${ }^{143,144}$ and the immune reaction to PGL-I is often used as a prognostic marker for early infections with leprosy. ${ }^{307}$ Recent reports have suggested PGLs 
produced by M. tuberculosis as the cause of hypervirulence in isolates from the $\mathrm{W}$ Beijing lineage. ${ }^{153}$ Other recent studies, however, indicated that while PGL can modulate the host immune response and suppress production of Th2 cytokines, they do not confer hypervirulence in their own. ${ }^{174}$

Biosynthesis of PGL requires multiple enzymes in different pathways. These enzymes include PKS and fatty acid synthases involved in phthiocerol and mycocerosic acid biosynthesis, certain synthases like 4HB-synthase responsible for the production of 4hydroxybenzoic acid, ${ }^{308}$ glycosyl transferases which transfer of the sugar residues to the aglyon, ${ }^{152}$ and PKS1-15 which links the aromatic residue to the fatty acid chain. ${ }^{150}$ Most strains of M. tuberculosis are incapable of producing PGL due to a frame shift in the $p k s$ $1-15$ gene. ${ }^{147,150}$ Meanwhile, all strains of $M$. tuberculosis have the capability of producing PDIM and some forms of glycosylated benzoic acid esters like those detected by Perez and colleagues in the culture filtrate of M. tuberculosis $\mathrm{H}_{37} \mathrm{Rv} .{ }^{152}$

In this study we investigated the production of PGL by different MDR clinical isolates collected from South Korea by the National Institute of Allergy and Infectious Diseases (NIAID) collected by Dr. Clifton E. Barry III and his research group. We also included some laboratory strains and other clinical isolates from the collection of the State of Tennessee health department. We used NMR-based lipid profiling to determine the production and the relative abundance of PGL in these strains and in the same time ensure stability in the concentration of other cellular lipids.

\subsubsection{Materials and Methods}

\subsubsection{Growth conditions and lipid extraction}

M. tuberculosis isolates were grown in Middlebrook 7H9 supplemented with $0.2 \%$ ${ }^{13} \mathrm{C}_{6}$-dextrose, $0.2 \%{ }^{13} \mathrm{C}_{3}$-glycerol, $0.05 \%$ Tween 80 and $5 \%$ BSA at $37{ }^{\circ} \mathrm{C}$ with continuous shaking at $250 \mathrm{rpm}$. 94 isolates were used for this study including 85 from South Korea, 5 from the state of Tennessee, in addition to H37Rv, CSU93, HN878 and its $\Delta$ pks mutant stain. Cells were harvested by centrifugation at $3700 \mathrm{xg}$ for 10 minutes. Cell pellets were washed and extracted as previously described under section 3.2.1.

\subsubsection{Isolation of PGL from M. liflandii}

M. liflandii cells were grown to stationary phase in Middlebrook 7H9, harvested by centrifugation, and subsequently extracted with ethanol. The ethanolic extract was dried in vacuo and the dried residue ( $80 \mathrm{mg}$ ) was re-suspended in $\mathrm{CHCl}_{3}$ and applied to a Biotage SP1 flash chromatography system equipped with a Biotage Si 12+M silica gel column. The column was eluted using a $\mathrm{CHCl}_{3}-$ to- $-\mathrm{CHCl}_{3} / \mathrm{CH}_{3} \mathrm{OD} 9: 1(\mathrm{v} / \mathrm{v})$ linear gradient. The major PGL was detected using silica TLC analysis with $\mathrm{CHCl}_{3} / \mathrm{CH}_{3} \mathrm{OH}$ $(95: 5, \mathrm{v} / \mathrm{v})$ as the developing system $\left(\mathrm{R}_{\mathrm{f}}=0.7\right)$. Lipids were visualized using cerric ammonium sulfate in $2 \mathrm{M}$ sulfuric acid after charring. Fractions containing pure PGL were pooled and dried in vacuo. 


\subsubsection{NMR acquisition and data processing}

The 2D-HSQC spectra were recorded using Varian Inova 500MHz spectrometer at $25^{\circ} \mathrm{C}$ degrees. The $2 \mathrm{D}-\mathrm{HSQC}$ spectra were acquired for 256 increments and 60 transients using a $5 \mathrm{~mm}$ trpfg sample probe or 10 transients using $3 \mathrm{~mm}$ internal flow cell probe. For all NMR acquisitions, samples were shimmed, toned and other acquisition parameters were optimized. NMR data were processed using either VNMR or Felix-NMR software. Biomarker signals for different lipids were selected and their integral volumes were measured $\left(\delta^{1} \mathrm{H}=7.74, \delta^{13} \mathrm{C}=133.64 \mathrm{ppm}\right.$ for menaquinone, $\delta^{1} \mathrm{H}=5.26, \delta^{13} \mathrm{C}=70.74$ for $\mathrm{PL}$, $\delta^{1} \mathrm{H}=5.30, \delta^{13} \mathrm{C}=69.31$ for TAG, $\delta^{1} \mathrm{H}=0.67, \delta^{13} \mathrm{C}=15.76$ for $\mathrm{TDM}, \delta^{1} \mathrm{H}=1.23, \delta^{13} \mathrm{C}=45.54$, for PIM and $\delta^{1} \mathrm{H}=5.13, \delta^{13} \mathrm{C}=93.65$ for ATs. Integration values obtained for different isolates were compared to the corresponding values in M. tuberculosis $\mathrm{H}_{37} \mathrm{Rv}$ after normalization to the integration value of the menaquinone signal which was used as an internal reference. The values of PL biomarker signals were used as a secondary reference to insure the quality of the data.

\subsubsection{Results and Discussion}

\subsubsection{Selection for biomarker signals for PGL}

Before screening for PGL production, it was necessary to define the set of biomarker signals to be used for this purpose. In choosing these biomarkers signals, it is important that they will not overlap with other signals from other lipid molecules. It is also essential to ensure that these signals are unique for the targeted molecule. Based on this latter criterion and upon considering what is known about the lipidome of M. tuberculosis, most signals in the phthiocerol part of PGL can not be considered as biomarker signals because they are present in PDIM as well. For similar reasons, most of the signals of the glycon part are not suitable because they are very similar to signals from the glycosylated benzoic acid esters produced by some M. tuberculosis strains. However, signals of the phenolic ring and the adjacent methylene (benzylic protons) can be of special utility in detection of PGL for several reasons. First, few mycobacterial lipids have aromatic nucleus thus the phenolic and benzylic signals are likely to fall in well-resolved area of the spectrum. Second, these signals appear at the junction between the glycon and aglycon part of PGL, therefore, the chemical shifts for these protons will uniquely reflect the chemical environment created by that link which is specific to PGLs. Third, the gene responsible for establishing this link, pks 1-15, can be considered as the genetic marker for PGL production within Mycobacteria and among different strains of M. tuberculosis. Finally, a set of a parent and $\Delta p k s 1-15$ mutant stains is available and can be used to demonstrate the possibility of PGL detection and ensure the right assignment of the PGL signals.

\subsubsection{Use of isolated standard for the assignment of PGL biomarker signals}

A major obstacle in studying M. tuberculosis PGL is their relative low abundance when compared with other lipid molecules. Chemical structures of $M$. tuberculosis PGL 
were proposed based upon ${ }^{1} \mathrm{H}-\mathrm{NMR}$ experiment and glycosidic linkage analysis by GC/MS. To assign PGL signals in the 2D-HSQC spectrum it is important to have the chemical shifts for both the proton and carbon atoms in PGL obtained from a 2Dspectrum acquired under similar conditions to that used for our lipid profiling protocol. However, it not practical to grow large amounts of cells to isolate enough PGL for 2DHSQC structural assignment. To solve this issue, we used the isolated PGL produced by M. liflandii as our preliminary reference to assign most of the PGL signals in the lipid profile of M. tuberculosis HN878. As expected, signals for the phenolic rings and the benzylic carbon were well isolated. The two $p$-aromatic $\mathrm{CH}$ were detected at $\delta^{1} \mathrm{H}=7.02$, $\delta^{13} \mathrm{C}=117.6 \mathrm{ppm}$ and $\delta^{1} \mathrm{H}=7.18, \delta^{13} \mathrm{C}=129.7 \mathrm{ppm}$ while the benzylic protons were detected at $\delta^{1} \mathrm{H}=2.58, \delta^{13} \mathrm{C}=46.8 \mathrm{ppm}$ (Figure 5.7). To confirm the assignment, the lipid profile of HN878 was compared to that HN878 $\Delta p k s 1-15$, a mutant strain where the pks 115 gene has been disrupted rendering the strain incapable of producing PGL. The two profiles were quite similar except for the absence of PGL signal from HN878 $\Delta p k s$ 1-15 profile. These biomarker signals could not be detected also in the lipid profiles of $M$. tuberculosis $\mathrm{H}_{37} \mathrm{RV}$ and the CDC-1551 strains.

\subsubsection{Screening of other clinical isolates using 2D-NMR}

In the previous experiments, lipid extract of CDC1551, HN878 wt and its $\Delta p k s 1-15$ mutant were obtained from $30 \mathrm{mls}$ culture $(150 \mathrm{mgs})$ and the corresponding lipid profiles were developed by prolonged NMR acquisition for 60 transients. For screening purposes, both the initial cell pellet weight and the NMR acquisition time have to be reduced. Some of the clinical isolate do not exhibit optimal growth behavior and the long NMR acquisition time will be inefficient when dealing with a large number of samples. To produce good quality spectra from smaller samples, we used $3 \mathrm{~mm}$ NMR sample probe which effectively reduced the sample volume required to $160 \mu 1$. Such volumes can be obtained from 10mls of cell culture grown to the mid exponential phase. Differences observed in 2D-profiles between HN878 and its $\Delta p k s 1-15$ mutant obtained using the $5 \mathrm{~mm}$ probe were replicated using the $3 \mathrm{~mm}$ sample probe in a shorter acquisition time of about 1 hour 40 minutes.

Lipid extracts from $94 \mathrm{M}$. tuberculosis isolates were obtained from a $10 \mathrm{mls}$ of cell culture as described previously and an HSQC lipid profile was obtained for each using $3 \mathrm{~mm}$ NMR sample probe. Immediately, two isolates (OA033 and OB239) were disregarded from further analysis after being identified as $M$. avium isolates by this method. Overall, the quality of the spectra was satisfactory except in few cases (6 isolates out of 94 tested).

Isolates from South Korea were easily confirmed to be from the W-Beijing strain by the abundance of their TAG compared to $\mathrm{H}_{37} \mathrm{Rv}$ strain. PGL signals were detected in the lipid profile of 48 cases of the 85 South Korean isolates and in none of the Tennessee State isolates. It was also observed that when compared to the $\mathrm{H}_{37} \mathrm{Rv}$, each of the two different isolate collections displayed a specific pattern. While the Korean isolates displayed high levels of TAG (three times or higher), they also showed significantly lower levels of PIMs and ATs (at 40\% their levels in $\mathrm{H}_{37} \mathrm{Rv}$ ). On the contrary, all tested 

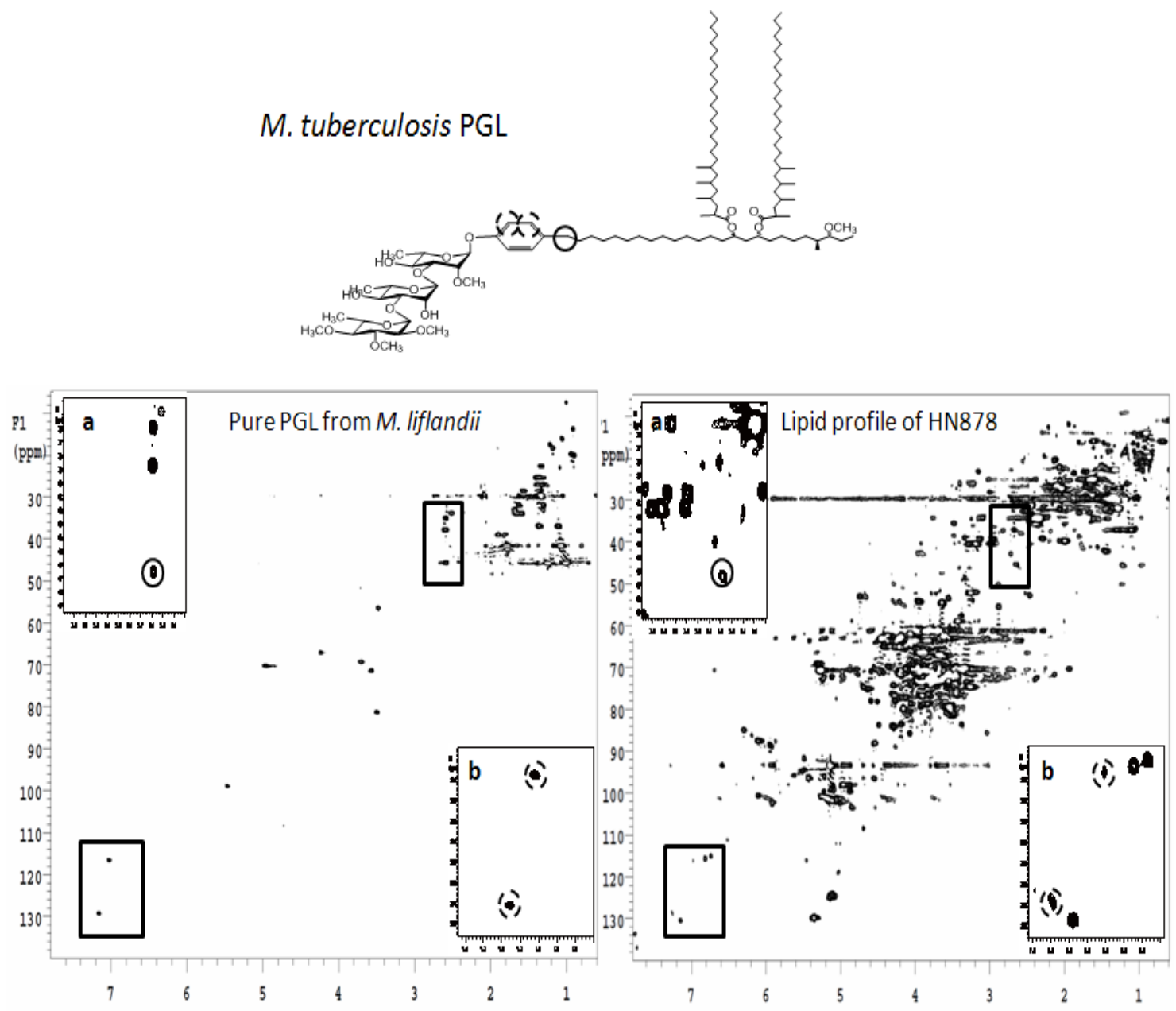

Figure 5.7 Selection of Biomarker Signals of M. tuberculosis PGL

A monosaccharide PGL isolated from $M$. liflandii was used to assign PGL signals in the 2D-HSQC lipid profile of M. tuberculosis HN878. Inserts, marked as (a), show partial windows in both the PGL standard and the HN878 lipid profile where the benzylic signals at $\delta^{1} \mathrm{H}=2.58, \delta^{13} \mathrm{C}=46.8 \mathrm{ppm}$ of PGL are highlighted by solid circles. Inserts marked as (b), represent a partial windows where the aromatic signals of PGL at ${ }^{1} \mathrm{H}=7.02, \delta^{13} \mathrm{C}=117.6 \mathrm{ppm}$ and $\delta^{1} \mathrm{H}=7.18, \delta^{13} \mathrm{C}=129.7 \mathrm{ppm}$ have been highlighted in dashed circles in both spectra. 
Tennessee isolates showed significantly lower levels of TAG ( $2-15 \%$ of $\mathrm{H}_{37} \mathrm{Rv}$ levels) and to less extent lower levels of TDM (30-50\% of $\mathrm{H}_{37} \mathrm{Rv}$ levels), while ATs, PIMs and PDIM were detected at comparable levels. Full results are outlined in Table 5.4.

Results from this screening are, to some extent, in agreement with the suggestion that PGLs may be, at least partially, responsible for the hypervirulence of the W-Beijing lineage as we were only able to detect PGL in strains that belong to this lineage. However, our screening results suggest that other factors might participate in such phenomenon since PGL were not detected at measurable levels in many of these isolates. In addition, we have found some other differences between of the lipid composition of the W-Beijing strains and strains that were obtained from other areas.

\subsection{Conclusion}

Different spectroscopic and chromatographic techniques can be used for the targeted analysis of a specific lipid species. The selection of the analytical method to be used depends largely on the properties of the molecule to be investigated. Molecules that are readily ionizable are better analyzed via mass spectrometry while molecules that have low ionization potential are bad candidate for such analysis. In the later case, the analysis can be achieved through HPLC, GC or NMR. GC analysis requires that the target molecules must show some degree of volatility or to be derivatized before analysis to volatile derivatives. Depending on the type of HPLC detector used for analysis, HPLC analysis may require certain characteristics to be built in the targeted lipid species. For example, the most commonly used HPLC detector, UV-detector, requires a UV absorbing functionality to be present or to be added to the molecule targeted for analysis. NMR on the other hand has more universal detection but due to its low sensitivity it is better suited for the analysis of relatively abundant cellular components. Certain measures are also to be implemented during analysis of complex mixtures to insure good resolvability of the data points as well. In all cases, some effort has to be directed towards the standardization and optimization of the chosen strategy to enhance sensitivity towards the detection of the targeted molecules.

In this chapter, two chemically different molecules were targeted for screening in the lipid extract of different Mycobacteria and two different methods were chosen for this purpose accordingly. The first example, mycolactone, represents a virulence factor of a polyketide nature that is usually produced by MPM in relatively small amounts. Mycolactones are easily ionizable as sodium adduct and hence are great candidate for MS analysis. Moreover, MS analysis of complex lipid extract can easily identify analogues of mycolactone due to the presence of a distinct fragment that represent the conserved lactone ring at $\mathrm{m} / \mathrm{z} 429$. All mycolactones share also consistent fragmentation pathways Knowledge of these pathways can help in determining certain structural features of mycolactone as it is the case with our analysis of mycolactone $\mathrm{D}$ and other mycolactones isolated from M. ulcerans subsp shinshuense. 
Table 5.4 Results from Screening M. tuberculosis Clinical Isolates for PGL Production

\begin{tabular}{|c|c|c|c|c|c|c|c|c|}
\hline \multirow[t]{2}{*}{ Strain } & \multicolumn{2}{|c|}{ PGL } & \multirow[t]{2}{*}{ TDM } & \multirow[t]{2}{*}{ TAG } & \multirow[t]{2}{*}{ PDIM } & \multirow[t]{2}{*}{ AT } & \multirow[t]{2}{*}{ PIM } & \multirow[t]{2}{*}{ Remarks } \\
\hline & aromatic & benzylic & & & & & & \\
\hline A29 & + & + & $=$ & +++ & $=$ & - & - & PGL +ve \\
\hline B019 & + & + & - & - & $=$ & - & $=$ & PGL +ve \\
\hline B076 & & & + & +++ & - & - & - & PGL +ve \\
\hline B122 & & & - & - & - & - & - & Bad quality \\
\hline B148 & & + & $=$ & +++ & - & - & $=$ & \\
\hline B183 & & + & + & +++ & $=$ & $=$ & + & PGL +ve \\
\hline OA002 & + & + & $=$ & +++ & - & - & - & PGL +ve \\
\hline OA004 & & & - & - & - & - & - & Bad quality \\
\hline OA015 & & & ++ & +++ & $=$ & - & - & \\
\hline OA022 & & & - & +++ & - & - & - & \\
\hline OA023 & ++ & ++ & $=$ & +++ & - & - & - & PGL +ve \\
\hline OA025 & & + & +++ & +++ & - & - & - & PGL +ve \\
\hline OA029 & ++ & + & - & + & - & - & - & PGL +ve \\
\hline OA030 & + & + & $=$ & +++ & $=$ & $=$ & $=$ & PGL $+\mathrm{ve}$ \\
\hline OA033 & Signals a & $\delta^{1} \mathrm{H}=7.2, \delta c$ & $=128.71$ & and $\delta 1 \mathrm{H}=$ & $=4.67, \delta \mathrm{C}$ & $=55.36$ & ppm & M. avium \\
\hline OA039 & ++ & ++ & $=$ & +++ & - & - & - & PGL +ve \\
\hline OA040 & & + & - & - & $=$ & - & - & Bad quality \\
\hline OA046 & & + & +++ & +++ & +++ & - & - & PGL +ve \\
\hline OA049 & & + & +++ & +++ & - & - & $=$ & PGL +ve \\
\hline OA054 & ++ & ++ & $=$ & +++ & - & - & - & PGL +ve \\
\hline OA055 & & + & $=$ & +++ & - & - & - & \\
\hline OA057 & ++ & ++ & $=$ & +++ & - & - & - & PGL +ve \\
\hline OA062 & + & + & +++ & +++ & $=$ & - & - & PGL +ve \\
\hline OA071 & & + & $=$ & +++ & + & - & $=$ & \\
\hline OA074 & & + & $=$ & +++ & $=$ & - & - & \\
\hline OA081 & & & - & - & - & - & - & Bad quality \\
\hline OA086 & & + & $=$ & +++ & $=$ & - & - & \\
\hline OA090 & + & + & $=$ & +++ & - & - & - & PGL +ve \\
\hline OA094 & ++ & + & $=$ & +++ & - & - & - & PGL +ve \\
\hline OA108 & + & + & $=$ & + & +++ & $=$ & + & PGL +ve \\
\hline OA109 & + & + & + & +++ & $=$ & - & - & PGL +ve \\
\hline OA110 & & & - & - & - & - & - & Bad quality \\
\hline OA116 & ++ & ++ & $=$ & ++ & - & - & - & PGL +ve \\
\hline OA119 & + & + & $=$ & +++ & - & - & - & PGL +ve \\
\hline OA121 & & & - & + & - & - & - & Bad quality \\
\hline OA122 & ++ & ++ & + & +++ & - & - & - & PGL + ve \\
\hline OB012 & & + & $=$ & +++ & - & - & $=$ & PGL +ve \\
\hline OB013 & & + & + & +++ & $=$ & - & - & \\
\hline
\end{tabular}


Table 5.4 (continued)

\begin{tabular}{|c|c|c|c|c|c|c|c|c|}
\hline \multirow[t]{2}{*}{ Strain } & \multicolumn{2}{|c|}{ PGL } & \multirow[t]{2}{*}{ TDM } & \multirow[t]{2}{*}{ TAG } & \multirow[t]{2}{*}{ PDIM } & \multirow[t]{2}{*}{$\mathbf{A T}$} & \multirow[t]{2}{*}{ PIM } & \multirow[t]{2}{*}{ Remarks } \\
\hline & aromatic & benzylic & & & & & & \\
\hline OB016 & & + & $=$ & +++ & - & - & - & \\
\hline OB023 & ++ & ++ & $=$ & ++ & $=$ & - & $=$ & PGL +ve \\
\hline OB026 & ++ & ++ & - & +++ & - & - & - & PGL +ve \\
\hline OB047 & & + & $=$ & +++ & $=$ & - & - & \\
\hline OB051 & & + & $=$ & +++ & - & - & - & \\
\hline OB059 & ++ & ++ & $=$ & +++ & $=$ & - & - & PGL +ve \\
\hline OB066 & + & + & ++ & +++ & $=$ & - & - & PGL +ve \\
\hline OB074 & & + & $=$ & + & - & - & - & \\
\hline OB075 & & & + & ++ & $=$ & $=$ & - & \\
\hline OB076 & ++ & ++ & $=$ & +++ & $=$ & - & - & PGL +ve \\
\hline OB078 & + & + & $=$ & +++ & + & - & - & PGL +ve \\
\hline OB085 & + & + & + & +++ & - & - & - & PGL +ve \\
\hline OB096 & & + & - & +++ & $=$ & - & - & \\
\hline OB099 & + & + & $=$ & ++ & - & - & - & PGL +ve \\
\hline OB104 & + & + & $=$ & +++ & $=$ & - & - & PGL+ve \\
\hline OB115 & ++ & ++ & $=$ & ++ & - & - & - & PGL +ve \\
\hline OB123 & + & + & $=$ & +++ & $=$ & - & - & PGL +ve \\
\hline OB124 & ++ & ++ & $=$ & ++ & - & - & - & PGL +ve \\
\hline OB125 & & + & $=$ & + & $=$ & - & - & Bad quality \\
\hline OB126 & & + & $=$ & ++ & - & - & - & \\
\hline OB127 & + & + & - & + & - & - & - & PGL +ve \\
\hline OB139 & + & + & $=$ & +++ & - & - & - & PGL +ve \\
\hline OB140 & ++ & ++ & $=$ & +++ & $=$ & - & - & PGL +ve \\
\hline OB142 & & + & - & +++ & - & - & - & \\
\hline OB143 & + & + & $=$ & +++ & - & - & - & PGL +ve \\
\hline OB145 & + & + & $=$ & +++ & $=$ & - & - & PGL +ve \\
\hline OB154 & & + & $=$ & +++ & - & - & - & \\
\hline OB156 & & + & $=$ & ++ & - & - & - & \\
\hline OB157 & & + & ++ & +++ & $=$ & - & $=$ & \\
\hline OB158 & + & + & $=$ & + & - & - & - & PGL +ve \\
\hline OB161 & & + & $=$ & ++ & - & - & - & \\
\hline OB170 & & + & $=$ & +++ & $=$ & - & - & \\
\hline OB178 & & + & - & + & $=$ & - & - & \\
\hline OB179 & ++ & ++ & ++ & +++ & - & $=$ & $=$ & PGL +ve \\
\hline OB185 & ++ & ++ & ++ & +++ & $=$ & $=$ & $=$ & PGL +ve \\
\hline OB192 & + & + & $=$ & +++ & - & - & - & PGL +ve \\
\hline OB202 & ++ & ++ & + & +++ & - & $=$ & - & PGL +ve \\
\hline OB206 & & & $=$ & +++ & $=$ & - & - & \\
\hline OB210 & & + & $=$ & +++ & $=$ & - & - & \\
\hline
\end{tabular}


Table 5.4 (continued)

\begin{tabular}{|c|c|c|c|c|c|c|c|c|}
\hline \multirow[t]{2}{*}{ Strain } & \multicolumn{2}{|c|}{ PGL } & \multirow[t]{2}{*}{ TDM } & \multirow[t]{2}{*}{ TAG } & \multirow[t]{2}{*}{ PDIM } & \multirow[t]{2}{*}{ AT } & \multirow[t]{2}{*}{ PIM } & \multirow[t]{2}{*}{ Remark } \\
\hline & aromatic & benzylic & & & & & & \\
\hline OB218 & & & - & +++ & - & - & - & Bad quality \\
\hline OB227 & + & + & $=$ & +++ & - & - & - & PGL +ve \\
\hline OB228 & + & & $=$ & ++ & - & - & - & \\
\hline OB229 & + & + & - & + & - & - & - & PGL +ve \\
\hline OB231 & + & + & $=$ & ++ & $=$ & - & - & PGL +ve \\
\hline OB235 & & + & $=$ & +++ & $=$ & - & - & \\
\hline OB239 & \multicolumn{7}{|c|}{ Signals at $\delta^{1} \mathrm{H}=7.2, \delta \mathrm{C}=128.71$ and $\delta 1 \mathrm{H}=4.67, \delta \mathrm{C}=55.36 \mathrm{ppm}$} & M. avium \\
\hline CSU93 & & & $=$ & - & $=$ & + & $=$ & \\
\hline HN878 & ++ & ++ & $=$ & +++ & + & $=$ & - & PGL +ve \\
\hline $878 \Delta \mathrm{pks}$ & & & $=$ & ++ & $=$ & $=$ & - & \\
\hline TN15 & & & - & - & $=$ & + & - & \\
\hline TN18 & & & - & - & $=$ & $=$ & $=$ & \\
\hline TN22 & & & $=$ & - & - & $=$ & + & \\
\hline TN26 & & & - & - & $=$ & $=$ & $=$ & \\
\hline TN29 & & & - & - & - & $=$ & $=$ & \\
\hline
\end{tabular}

Signal intensities for lipid molecules other than PGL are reported in comparison to $\mathrm{H}_{37} \mathrm{Rv}$. (=), comparable to $\mathrm{H}_{37} \mathrm{Rv}$ at $60 \%-140 \%$ value, $(+)$ at $140 \%-200 \%$ value, $(++)$, at $200 \%-300 \%$ value, $(+++)$ at more than $300 \%$ value, and (-) at less than $60 \%$ of that signal in H37Rv. Intensities of different biomarker signals were measured and normalized as described in section 5.2.2.3. Samples were judged to be PGL positive if more than one of the biomarker signals of PGL were detected in their corresponding spectra. 
Due to the presence of a polyene side chain in mycolactones, they are also good candidates for HPLC-UV analysis. The absorption wavelength of these molecules at $360 \mathrm{~nm}$ is relatively uncommon for other lipid molecules hence it is possible to use such a technique for mycolactone detection in total lipid extract. In addition, HPLC can succeed where MS analysis fail. While MS detect all geometric isomers as a single entity of the same mass, a well-developed HPLC gradient can differentiate between geometric isomers and provide information that are not attainable through MS analysis alone.

In the second case, screening for PGL represents a different challenge. The molecular mass of PGL is not a fixed value due to the possibility of the variation in the aglycon part. Both mycoserosic acid and phenolphthicerol exhibit variations in their structures in both chain length and the number of substituent. This variability causes great variation in the possible molecular weight of PGL. These molecules are also very hydrophobic in nature and their ionization under most MS ionization techniques is considerably poor.

2D-HSQC-based screening for PGL that we used here was quite successful in several aspects. We were able to determine biomarker signals that can be used for different analogues of PGL irrespective of the variation in their corresponding mass. We were also able to detect PGL in clinical isolates using only 10 milliliters of cell culture (and as low as $0.75 \mathrm{mg}$ of dried lipid extract) in a relatively short time period. While screening for PGL in these samples, all other cell wall lipids can be measured simultaneously. This adds a great value to the data obtained as it can point out in some case to certain metabolic coupling processes. Unfortunately, some clinical isolates showed slow and erratic growth behavior which affected the amount of lipids available for analysis. In some cases, the lipid profiles were of such bad quality that deterred conclusive data interpretation. Due to the relatively low abundance of PGL in some isolates, in certain cases only one or two biomarker signals were detected for PGL instead of all three. Hence, a threshold based on multiple referencing is needed as an important measure to insure the quality of the spectrum and validate the conclusion drawn from it. 


\section{CHAPTER 6. SUMMARY AND CONCLUSION ${ }^{4}$}

\subsection{The Metabolomics Research Field}

\subsubsection{The "Omics" Era}

The introduction of global analysis techniques came as a natural sequel of our ability to decipher a whole genome sequence. With the availability of complete genomic sequence of several organisms, the "omics" era began with the introduction of microarrays in $1995^{309}$ as a tool to investigate the cellular transcriptome. Shortly after, the concept of proteome as the protein complement of the genome was introduced ${ }^{310}$ and followed by the introduction of the term proteomics in $1997 .{ }^{311}$ Several techniques have been introduced since then for these purposes and several modifications and improvements have followed. The result was unprecedented flood of information about transcription and translation regulation during different stages of cell cycle and in response to different environmental cues.

As researchers became accustomed to have a panoramic view of the cellular mRNA and the protein pool, the need to develop a parallel view of the cell metabolites became apparent. The term metabolome was introduced to describe the pool of small organic molecules that include cell metabolites, metabolic intermediates and small molecules involved in cellular signaling. ${ }^{312}$ The parallel terms metabolomics and metabonomics were then coined to describe the measurement of all endogenous metabolites in a cell or a body fluid. Changes in cellular metabolites can be regarded as the end product of the regulatory response of the cell to environmental stimuli. ${ }^{313-315}$ In that sense, metabolomics should provide a more direct representation of cellular events than those offered by genomics and proteomics. ${ }^{316}$

In Mycobacteria research, the "omics" era started with the completion of the whole genome sequencing of $M$. tuberculosis in $1998 .^{221}$ Short after, whole genome sequences of different Mycobacteria species and strains have also been completed. ${ }^{222,257,317}$ Microarrays and proteomics techniques have been successfully used in Mycobacteria research. Some interesting examples include the use of RNA microarrays to identify the mode of action of several anti-TB drugs. ${ }^{276,318,319}$ Through the use of such techniques, researchers have also identified the role that isocitrate lyase and nitrate reductase play in the metabolic shift of Mycobacteria to latency. ${ }^{275,277,320}$ Despite the availability of such information, we are still represented with the great challenge of deciphering the genome into the ultimate end products, the metabolome.

\footnotetext{
${ }^{4}$ Parts of this chapter are adapted with permission. Mahrous, E.A., Lee, R.E. \& Lee, R.E. A rapid approach to lipid profiling of Mycobacteria using 2D HSQC NMR maps. J Lipid Res. 49, 455-63 (2008). ${ }^{240}$
} 


\subsubsection{Challenges in Metabolomics}

However, there has been limited development in the metabolomic field and it largely seems to be lagging behind the great advances of genomics and proteomics. This can be attributed in part to the relatively recent interest in the field and the many technical challenges it faces. Metabolomic studies include two main stages; first, the extraction of the cell metabolites then the detection of these small molecules in the extract which may require a prior step of chromatographic separation. ${ }^{321}$ The great diversity of the cell metabolites in terms of cellular abundance and chemical nature (carbohydrates, lipids, peptides and polyketides) represents a major challenge since there is no reliable extraction solvent or technique that can guarantee the extraction of all cell metabolites. On the other hand, most analytical methods do not have enough capability to deal with biological mixture with thousands of components of such diverse chemical structures and with wide range of cellular concentration. In fact, until now a combination of analytical methodologies has a limited power of analyzing few hundred metabolites which is a long way to go compared to microarrays that can depict the whole transcriptome. ${ }^{322}$

\subsubsection{The Field of Lipidomics}

In order to partially overcome the problem associated with the chemical diversity of the cell metabolome, it can be subdivided and analyzed as separate pools; lipid pool, peptide pool, etc. One of the most significant metabolite pools is that of the cellular lipid, the main focus of this dissertation. All lipidomics techniques follow a general scheme that start with extraction of the cellular lipid with an organic solvent from a whole organism, body fluid or tissue then the lipid pool is analyzed by either a chromatographic or spectrometric method to produce a "lipid profile."236 This lipid profile represents a panoramic view of cellular lipids which include among others; membrane lipids, cellular signaling molecules and biosynthetic intermediates. ${ }^{236}$

Genetic and metabolic differences in the starting materials, tissue or microorganism, should be reflected in their lipid profiles. The changes observed in these profiles when related to the initial variation of the analyzed samples are then used to formulate a hypothesis about a certain metabolic pathway. A combination of data obtained from such lipidomic approach together with data from genomics and proteomics can provide a complete picture of the cellular response and the regulatory process involved within. In that sense, it was encouraging that we were able to produce results that came in agreement with other observation obtained through molecular biology techniques. For example, our lipidomics characterization of $M$. liflandii was consistent with what is known so far about its evolutionary relation to M. marinum and M. ulcerans ${ }^{256,258}$ (section 3.3.4). Also, our conclusion that Mycobacteria overproduces TAG during its shift to dormancy came in agreement with previous results that showed an up-regulation in M. tuberculosis' TAG synthases when cells were subjected to low oxygen conditions. $^{278}$ 


\subsubsection{Designing a New Lipidomics Approach}

Before designing a new approach to address lipid profiling it is important to consider several factors. In the extraction process, effort has to be made in the selection of the solvent and the extraction methodology to insure that all cell lipids are included in the lipid extract without bias toward certain class of lipids over the other. Multiple extraction steps and the use of solvent mixtures instead of a single solvent can help to reduce such bias. Choice of the analytical method in lipid profiling should require only minimum manipulation and avoid partitioning of the total pool as much as possible to avoid loss of some lipids or duplicating their presence (but with lower concentration) in multiple profiles. Data analysis and management should include a statistical validation of the acquired data, a scheme to relate data to results from other "omics" techniques, a clear plan to develop a high throughput analysis and a depository mechanism to share the massive amount of data generated.

\subsection{New Techniques in Lipidomics}

Recent interest in lipidomics resulted in a vigorous search for the best tool to be used for such purpose. However, development of systems-level approaches for lipid analysis is still in its infancy and we have not reached the breakthrough that the genomic research arrived at with the development of microarray, or in proteomics through the 2Delectrophories/MALDI-MS approach. The old techniques of lipid analysis so often included radiolabeling or fluorescent labeling of lipid precursors followed by chromatographic separation of the components of the lipid extract. The relative ratio of the lipid pool that can be analyzed through these experiments depends on the choice of the lipid precursors and the extent of the label incorporation. The most common technique involves the use of radiolabeled ${ }^{32} \mathrm{P}$ - carbon precursors for the analysis of membrane phospholipids. ${ }^{236}$ However, with the great advancement of multiple analytical techniques, it became possible to develop more comprehensive and more accurate lipid analysis techniques.

\subsubsection{Mass Spectrometry (MS)}

Several developments in mass spectrometry have eliminated most restrictions on the use of MS in lipid analysis. First, the development of soft ionization techniques such as electrospray ionization ESI and MALDI have greatly reduced the possibility of ion fragmentation and thus provide more simplified spectra. ${ }^{199,323,324}$ MALDI offers an added advantage of its tolerance to electrolytes and salt contamination which reduce sample manipulation. Atmospheric pressure chemical ionization (APCI) can also be used for analysis of some non polar lipids that show some difficulty in producing ions. In addition, development of powerful analyzers such as time of flight (TOF) has greatly enhanced the sensitivity of MS analysis. 
On the other hand, there are some problems that restrict the use of MS-based lipidomics to certain areas. Most MS analysis techniques demonstrate their greatest utility in analyzing polar lipids such as phospholipids (in the case of ESI) and complex glycolipids and lipoproteins in the case of MALDI. ${ }^{323-326}$ Other problems can also be encountered especially in the analysis of crude lipid extracts such as ion suppression which hinder the analysis of low abundance lipid molecules. Quantitative MS analysis of lipids hence requires careful sample preparation and the incorporation of lipid standards to overcome ion suppression. ${ }^{236}$ Prior separation of the individual components of the lipid mixture by HPLC is the most widely used approach for efficient and quantitative analysis of lipid mixtures. ${ }^{327,328}$ An alternative approach, shotgun lipidomics, was introduced by Gross, R.W. ${ }^{329-331}$ This approach avoids prior HPLC separation of the crude lipid extract, rather it uses tandem mass scanning to develop two dimensional MS-fingerprints for the cellular lipidome. ${ }^{332}$ Hundreds of individual lipid molecules were analyzed and quantified through this technique which requires sub picomolar concentration of lipids for efficient analysis. ${ }^{329,332,333}$ For example, shotgun lipidomics was successfully used to link depletion of some cellular lipids to mitochondria dysfunction in diabetic myocardium. ${ }^{334}$

One of the most important applications of MS in lipidomoics is its use for targeted lipid analysis. Anionic lipids like phospholipids, gangliosides, sulfatides and fatty acids are great candidates for such studies. Through the development of triple quadropole analyzer, it is now possible to use MS to quantitatively monitor a single molecule in a complex lipid mixture using MS-MS sequences. ${ }^{325}$ Multiple reaction monitoring can provide an accurate and highly sensitive method to detect and quantify a specific molecule. ${ }^{327,335-337}$ This analysis, however, necessitates the incorporation of an internal standard and a prior knowledge of the fragmentation pathway of the targeted molecular ion. With the continuous introduction of new MS technology during the past two decades, MS has established itself as a major tool for lipidomics studies.

In our hands, MS analysis was especially helpful in the analysis of Mycolactones. These molecules are polyketide in nature and their structure is often decorated with multiple ionizable groups (section 5.1). We successfully used MS for the rapid screening and detection of mycolactones in the ASL of different Mycobacteria. However, our attempts to use mass spectrometry in the analysis of other lipid species (like TDM) were not as successful. The presence of lipid molecules as a series of analogues with variable carbon chain length often complicates the MS spectrum. Moreover, any minor contaminant with favorable ionizability can appear as a major component in an extract when compared to the low ionizability of cellular lipids. Certain measures have to be implemented to overcome this problem. ${ }^{215}$

\subsubsection{Chromatographic Separation Methods HPLC and GC}

Chromatography in general has preceded spectrometric methods and its development has initially enjoyed faster pace than that of MS and NMR. For this reason, chromatographic techniques, starting with TLC, have been used for metabolic analysis for several decades before spectroscopy. The simplicity of TLC and its low cost 
guaranteed its wide spread and frequent use by researchers. However, it suffers from low sensitivity and questionable resolution. Meanwhile, HPLC provides very quantitative measurement but most HPLC detectors are either irresponsive to most lipids (UV detector) or have low sensitivity (refractive index) ${ }^{236}$ The most commonly used HPLC detector for lipid analysis is MS but it usually necessitates certain treatment at the LC/MS interface to eliminate electrolytes and reduce the possibility of ion suppression. The technical advancement in development of universal detectors like evaporative light scattering and suppressed conductivity, was received with great enthusiasm from researches and they have been successfully used for global lipid analysis. ${ }^{338-340}$

Gas chromatography is most commonly used for analysis of fatty acids. Although analysis of lipids with GC requires a prior step of derivatization, the technique is very well established that the entire process is fully automated and is frequently used in the medical research field for diagnostic purposes. ${ }^{210}$ Other than fatty acid analysis, GC/MS can be used to help in the structural determination of some lipids particularly lipoproteins and glycopeptidolipids.

HPLC analysis was of a special use to us in our analysis of mycolactone (section 5.1.3.2). Due to the presence of mycolactones as equilibrating mixture of double bond isomers, MS analysis does not reveal such heterogeneity in the analyzed sample as all isomers have the same molecular weight. Moreover, the presence of such isomers can complicate the NMR spectrum and deter data interpretation. We have successfully used traditional UV-HPLC to understand the isomeric nature of different mycolactones. Others have used chiral HPLC column as a part of a complete synthetic strategy to assign the complete stereochemistry of different mycolactones as well. ${ }^{297}$

\subsubsection{NMR spectroscopy}

NMR represents a powerful analytical technique that offers a plethora of structural information. Although it has been well established a long time ago as an indispensable tool in organic chemistry, it keeps on offering researchers new opportunities in multiple and diverse fields. In lipidomics, beside its traditional role as a structural analysis tool, ${ }^{1} \mathrm{H}$ and ${ }^{32} \mathrm{P}-\mathrm{NMR}$ have often been used for global analysis of phospholipids. ${ }^{341,342}$ Theoretically, NMR is a quantitative and universal technique by nature, thus it offers great opportunities for application in the analysis of complex biological mixtures with chemically diverse components. Recently, it has been successfully used for some metabolomic studies. ${ }^{343,344}$

However for most biological research, the sensitivity of NMR is still considered to be unsatisfactory for many applications and it shows bias to abundant metabolites. For lipid analysis, structural membrane and energy storage lipids are good candidates for NMRbased lipid profiles due to their high natural abundance. While NMR analysis for less abundant lipids like cell signaling molecules does not seem to be feasible at this stage. With the vigorous effort deployed to advance NMR spectroscopy to its full potential, it is expected that it will be a major contributing factor in lipidomics studies. 
Our NMR methodology was quite successful in the analysis of the mycobacterial lipid pool considering that most of these lipids are structural components of the cell therefore they are relatively abundant. Most lipid classes previously reported in Mycobacteria were detected through this methodology regardless of their chemical class. However, when we started to fractionate our lipid pool into polar and nonpolar fractions, it became clear that more polar and less abundant lipids were underrepresented in the total lipid profile that was dominated by signals of TAG and fatty acids (section 2.3.6). So, it can be concluded that while all lipid classes were represented in our NMR lipid profile, the study of cellular lipids of low abundance remains challenging. Also, in drawing conclusion from these NMR profiles, the detection limit of the methodology has to be considered. For example in our PGL screening (section 5.2.3), we can firmly conclude that certain strains do produce PGL while we can not exclude its production by other strains considering its overall low abundance and the detection limits of the method.

\subsection{Summary of the Research}

\subsubsection{Method Development}

In the previous chapters, the development of a new NMR-based method for the global analysis of the Mycobacteria lipid pool was discussed. Several concepts were integrated to develop a complete approach that would provide mycobacteriology research with a new tool for investigating the role and the biochemical interaction of the cell wall components. The method is summarized as a one step $\mathrm{CD}_{3} \mathrm{OD}: \mathrm{CDCl}_{3}$ extraction of the $\mathrm{D}_{2} \mathrm{O}$ washed cells followed by 2D-HSQC NMR acquisition. The purpose of the method was to provide a global lipid analysis technique to complement the previously developed HR-MAS based analysis of the cell wall polysaccharides.

For such a technique to provide maximum utility, several factors were considered for method development. First, the simplicity of the procedure that includes only minimal sample manipulation and hence requires limited personal skills and minimizes artifacts during sample preparation. Second, the choice of the lipid profile format to be a simple 2D-HSQC NMR spectrum insures the possibility of wide application since it requires only a high field NMR spectrometer and a simple pulse sequence. Third, the enrichment

of the cell metabolites with ${ }^{13} \mathrm{C}$-isoptope eliminates some of the problems associated with NMR low sensitivity and greatly reduces the NMR acquisition time. Fourth, the fact that every molecule is represented by multiple signals rather than one signal, greatly reduces data ambiguity and positively increases confidence in data interpretation. Fifth, despite some complexity in the spectrum each molecule can be identified by at least one signal "biomarker signals", which can be assigned to each molecule either through literature search or through developing lipid profiles of isolated standard. Some highly congested area in the spectrum still can be resolved by three dimensional NMR experiments such as HCCH-TOCSY and HCCH-COSY in order to establish absolute clarity in data interpretation. Sixth, a semi-quantitative analysis of the signal can be achieved by normalization of biomarker signals intensity to that of a previously added standard. 
Finally, the method can provide a complementary approach to the HR-MAS profiling of the cell wall AG and LAM. ${ }^{189}$ Hence, from the same starting material $\left({ }^{13} \mathrm{C}\right.$-enriched cells $)$ a more complete metabolic picture can be developed by combining the two techniques; lipid profiling which provides a representation of cellular lipid and HR-MAS which provides a representation of cell wall AG and LAM.

\subsubsection{Method Validation}

The first validation of this NMR-based method came through developing a limited library of lipid profiles of some Mycobacteria species that have been thoroughly studied. As discussed in chapter.3, the lipid profiles of these species were reflective of the general features of Mycobacteria and also of the unique characteristics of certain species. When genes responsible for the production of a certain lipid were knocked out, signals corresponding to this lipid disappeared from the corresponding lipid profile of the mutant strains. For example, when pks 1-15 gene was knocked out in M. tuberculosis HN878, we observed the loss of the phenolic glycolipid signals in the lipid profile of the $\Delta p k s$ 1-15 mutant strain (section 5.2.3.2). Also in our first attempt to use NMR for mycolactone analysis, we observed the disappearance of mycolactone signals from the lipid profile of avirulent $M$. ulcerans $1615 \mathrm{~A}$ when it was compared to its parent strain, the virulent strain 1615. However, such results were obtained through MS analysis and HPLC profiling in much shorter time.

Prior to the use of such method for more quantitative analysis, certain criteria have to be established. These include the reproducibility of the data obtained from lipid profiles under fixed growth, extraction and acquisition conditions and the inclusion of a reference to facilitate the comparison between lipid profiles obtained by different personnel in different laboratories. In this work, we used a specific signal produced through the addition of a specific molecule (DMSO) to the lipid extract based on the weight of the cell pellet as a referencing point. A demonstration of the use of ${ }^{1} \mathrm{H}-{ }^{13} \mathrm{C} 2 \mathrm{D}-\mathrm{HSQC}$ for quantitative measurement of metabolites in plant extract has been already published, ${ }^{344}$ which opens the opportunities for similar use in bacterial metabolites. For spectral referencing, other small molecules can be used as long as they do not overlap with signals in the spectrum. Trimethoxybenzene (TMB) can be used for this purpose, however it will produce two discrete signals in two different area of the spectrum, which can then be used for the alignment of multiple spectra for comprehensive comparative analysis.

\subsubsection{Method Application}

The purpose of this research was to develop a new tool that will help researchers better understand the chemistry and biological function of the Mycobacteria cell components for the ultimate goal of defining new drug targets and developing new treatment option to control the spread of some devastating human and animal diseases. There are several areas where the integration of such methodology with other genetics 
approach can greatly advance our understanding of Mycobacteria metabolism, virulence and resistance. Some of the potential areas of method application are discussed below.

\subsubsection{Gene annotation}

The genome of Mycobacteria contains hundreds of genes that are involved in lipid biosynthesis and lipid metabolism and hundreds other that have no known function and need to be investigated for potential roles in survival and virulence for the purpose of identification of new molecular drug targets. ${ }^{221}$ Now with the availability of new molecular tools, a library of targeted mutant strains can be developed with relative ease and manageable cost. Screening of these libraries for changes in the lipid profiles should identify the lipid species affected by a specific mutation and offer the first steps to identify a role of the mutated gene in lipid biosynthetic pathways. Complementation of this lipid profiling technique with HR-MAS profiling of AG and LAM expand the area of metabolites that can be covered and facilitates the identification of genes involved in cell wall metabolism.

It is to be noted that changes observed in the HR-MAS and lipid profiles can be either direct effects due to investigated gene mutation or an indirect result due to cell adaptation to this mutation and accumulation of biosynthetic intermediates. Significant follow up investigation at both the genetic and biochemical levels is necessary to identify the direct gene function and distinguish it from other secondary effects. These secondary adaptive responses are nonetheless valuable in identifying points of conversions and diversions in the metabolic pathway and outlining compensatory mechanisms by which Mycobacteria respond to physiological stress due to gene mutation.

A direct demonstration of such application includes mutation of $R v 3792$ gene in $C$. glutamicum which resulted in disappearance of HR-MAS 2D-HSQC signals of all arabinan residues in the AG structure (Figure 6.1). In the lipid profile, however, some signals for acylated hexoses were observed in unusual abundance. Molecular biology and chemical analysis concluded that $R v 3792$ encodes a new arabinosyl transferase, designated aftA, that is responsible for transferring arabinose residue to the galactan core of AG macromolecule ${ }^{82}$ which is in total agreement with the results from the HR-MAS profile. From the lipid profile, it is apparent that the loss of a significant part of the structural integrity of the cell wall may be compensated for by the accumulation of hexose glycolipids.

\subsubsection{Drug action and drug resistance}

In vitro whole cell screening of chemical libraries is a useful strategy used by many investigators to identify new drug candidates that are effective against the tubercle bacilli. However, after identification of a viable drug candidate by this method comes the daunting task of deciphering the new drug's mode of action and its effects on cellular functions and metabolism. In the case of many anti-TB agents, this has never been an 

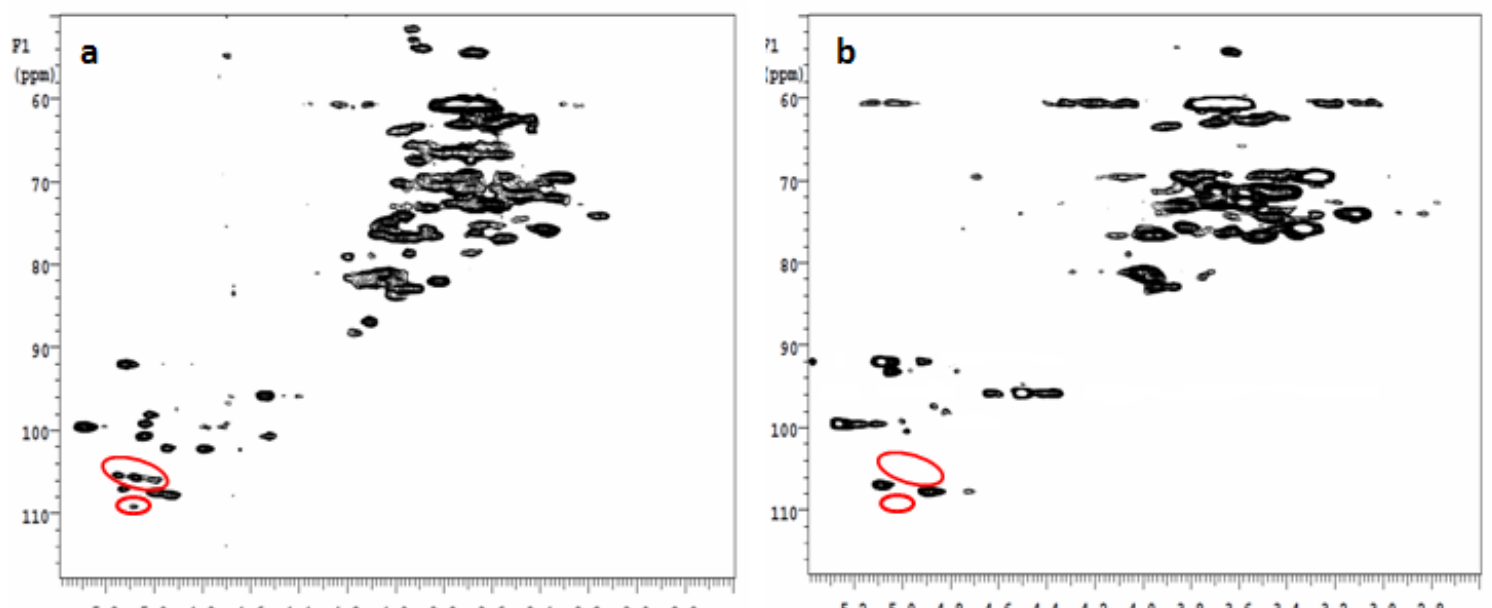

$\begin{array}{lllllllllllll}5.2 & 5.0 & 4.8 & 4.6 & 4.4 & 4.2 & 4.0 & 3.8 & 3.6 & 3.4 & 3.2 & 3.0 & 2.8\end{array}$

$\begin{array}{lllllllllllll}5.2 & 5.0 & 4.8 & 4.6 & 4.4 & 4.2 & 4.0 & 3.8 & 3.6 & 3.4 & 3.2 & 3.0 & 2.8\end{array}$
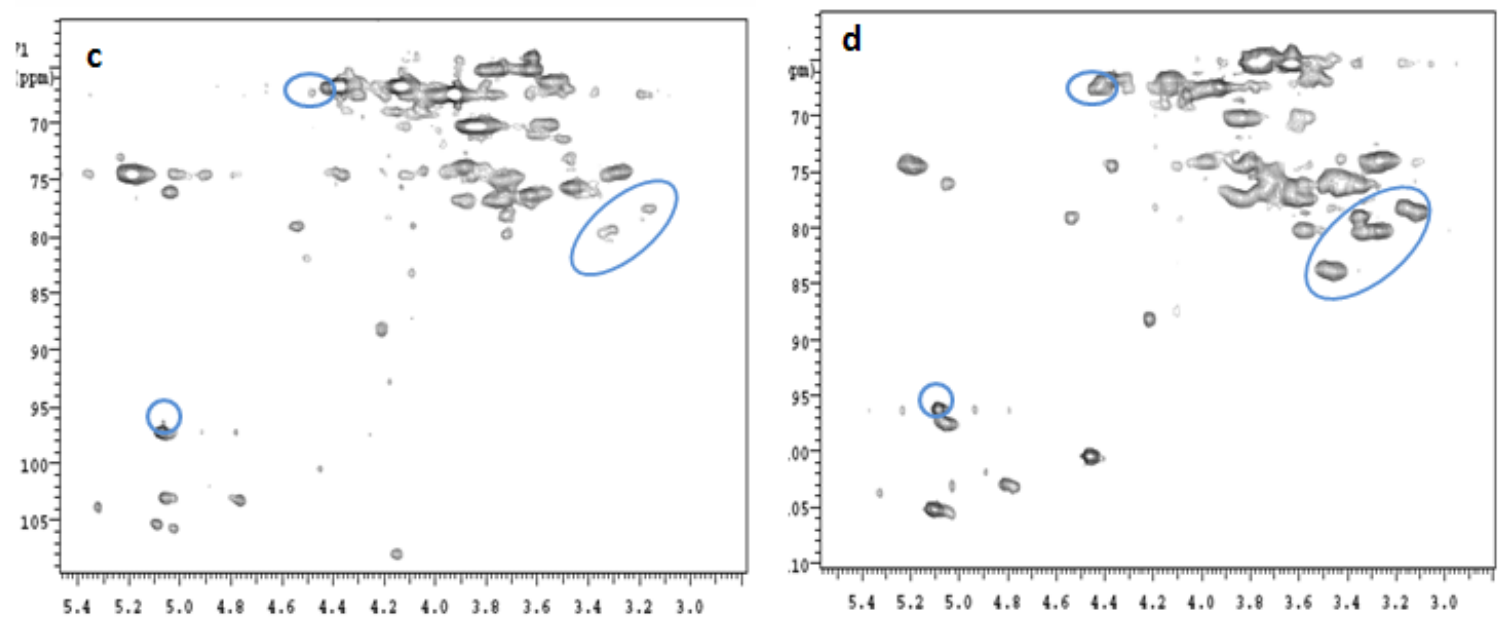

Figure 6.1 Effect of Gene Mutation on the Cell Wall of C. glutamicum

HRMAS profile of C. glutamicum wt, (a), and C. glutamicum $\Delta$ aftA, (b). Arabinan signals (circled in red) were totally absent from the HR-MAS profile of the mutant strain. Spectra (c) and (d) are partial windows of the 2D-HSQC lipid profile of C. glutamicum wt, (c), and the $\Delta$ aftA mutant, (d). Adaptive changes were observed upon mutation by the appearance of multiple signals for what appears to be a trehalose based glycolpids in the lipid profile of the $\Delta$ aftA mutant signals (circled in blue). 
easy task. For example, after the discovery of isoniazid in $1952^{21}$ by three different research groups, its mechanisms of action have been investigated by several groups as well. ${ }^{345-349}$ and several reasonable mechanisms of action have been reported. Although it is clear by now that INH has multiple cellular targets, it is unclear which of them is responsible for INH killing mechanisms. Ethambutol, another front line anti-TB drug, was discovered in 1961 through screening. ${ }^{350}$ Its mechanism of action has been widely speculated as well. ${ }^{345,346,351}$ Currently and after more than three decades of investigation, most researchers agree that the mechanism of action of ethambutol involves inhibition of arabinan biosynthesis, an event that leads to the inhibition of mycolic acids attachment to the cell wall and the subsequent accumulation of trehalose mycolates. ${ }^{67,352,353}$

We were able to reproduce these results following ethambutol treatment in $M$. smegmatis using both HR-MAS and lipid profiling methods in an unequivocal manner and in a very short period of time. Treatment of the cells with increasing concentration of ethambutol resulted in gradual disappearance of the branched structure of AG as indicated from the HR-MAS spectrum (Figure 6.2a-c). Meanwhile in the HSQC lipid profiles, there was a parallel gradual accumulation of trehalose dimycolate in the cellular lipid pool (Figure 6.2d-f). These results are in accordance with the reported literature and further underscore the argument for the mode of action of ethambutol through inhibition of arabinan synthesis. ${ }^{345,346,351}$

\subsubsection{Identification of virulence determinants}

Due to the worldwide spread of tuberculosis, there has been meticulous collection and documentation of many clinical strains. These clinical strains often demonstrate different transmitability, drug resistance and virulence. For example among the frequent outbreaks in different communities such as prisons, hospitals, schools and homeless shelters, different rates of transmission have been reported. ${ }^{354}$ An outbreak in clothing factories in Tennessee was associated with unusual high rates of transmission from the primary source as well as secondary sources. ${ }^{355}$ Investigation of the causative strains designated as CDC-1551 or CSU93 found out that although the strain displayed usual rates of initial implantation in a murine model, it was able to grow faster and to a lager extent than most laboratory strains which translates to a higher rate of transmission. ${ }^{355,356}$ Another strain responsible for an outbreak in a Texas prison, HN878, was found to be hypervirulant in mice when mice failed to induce appropriate immune response to the infection. ${ }^{357}$ In both cases, the variability in transmission and virulence was traced to specific changes in cell wall lipids. ${ }^{153,358}$

It is also well known that variable degrees of pathogenicity and virulence among different clades of $M$. tuberculosis can stem from certain structural polymorphism in its genome. ${ }^{357,359,360}$ Even of more interest to mycobacteriology scientists, is the notion that M. tuberculosis has evolved to develop strain compatibility to different human demography in different geographical regions. ${ }^{360,361}$ All of this genetic variability is ultimately represented as variability in cellular proteins and cellular metabolites and some of them can be detected by global analysis techniques, which include proteomics, metabolomics and lipidomics as well. 

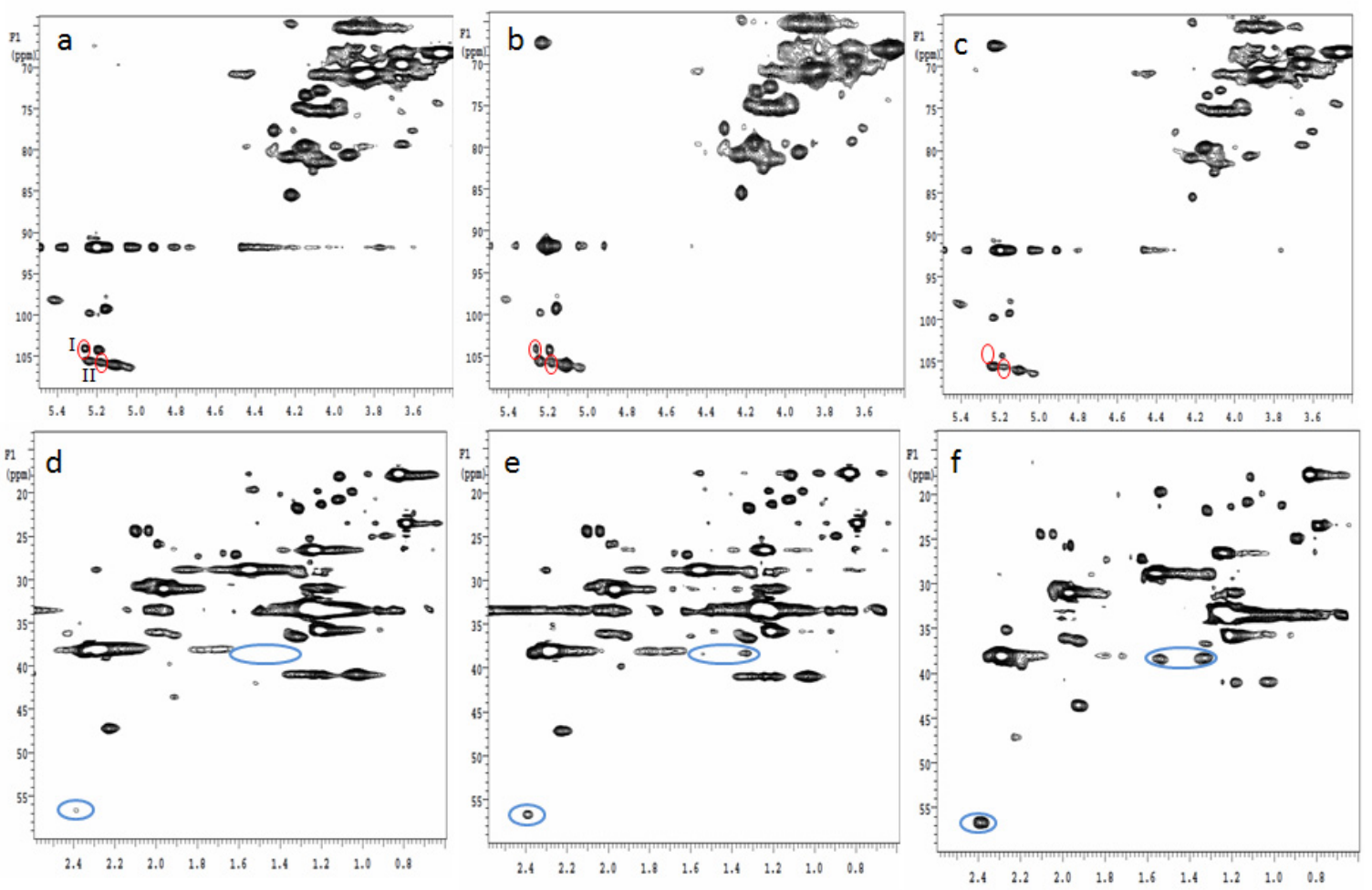

Figure 6.2 Effect of Ethambutol Treatment in the Cell Wall of M. smegmatis

Panels a-c, ${ }^{1} \mathrm{H}_{-}{ }^{13} \mathrm{C}$ HSQC HR-MAS spectra $\left(\delta^{1} \mathrm{H}, 3.4-5.3 \mathrm{ppm}, \delta^{13} \mathrm{C}, 64-110 \mathrm{ppm}\right)$ of live $M$. smegmatis cells in absence of ethambutol, (a), treated with ethambutol at $0.1 \mu \mathrm{g} / \mathrm{ml}$, (b), and $0.2 \mu \mathrm{g} / \mathrm{ml}$, (c). All spectra were referenced to the anomeric signal of 6$\beta$-Gal $f\left(\delta^{1} \mathrm{H} 5.28 \mathrm{ppm}, \delta{ }^{13} \mathrm{C} 106.98 \mathrm{ppm}\right)$. Gradual decrease in the intensity of signals corresponding to the branching of the Ara ${ }_{6}$ motif of $A G ; 3,5-\alpha-\operatorname{Ara} f(I)$ and 2- $\alpha$-Ara $f$ $\rightarrow 3$ (II) can be observed.

Panels d-f, ${ }^{1} \mathrm{H}-{ }^{13} \mathrm{C}$ HSQC spectra $\left(\delta^{1} \mathrm{H}, 0.6-2.6 \mathrm{ppm}, \delta^{13} \mathrm{C}, 13-60 \mathrm{ppm}\right)$ of total lipid extract from $M$. smegmatis cells in absence of ethambutol, (d), treated with ethambutol at $0.1 \mu \mathrm{g} / \mathrm{ml}$, (e), and $0.2 \mu \mathrm{g} / \mathrm{ml}$, (f). All spectra were referenced to the fatty acid terminal methyl signal $\left(\delta{ }^{1} \mathrm{H}=0.82 \mathrm{ppm}, \delta^{13} \mathrm{C}=17.82 \mathrm{ppm}\right)$. Gradual increase in intensity of signals corresponding to trehalose mycolates can be observed at $\left({ }^{1} \mathrm{H}=2.38 \mathrm{ppm}, \delta^{13} \mathrm{C}=56.73\right.$ ppm) and $\left({ }^{1} \mathrm{H}=1.32\right.$ and $\left.1.54 \mathrm{ppm}, \delta^{13} \mathrm{C}=38.17 \mathrm{ppm}\right)$. 
In our effort to use our NMR-based methodology to screen for certain virulence determinants, we screened more than 90 Mycobacteria tuberculosis isolates for PGL production. In the process of doing so, we were able to detect the production of PGL by a high percentage of clinical isolates from the W-Beijing lineage. Moreover, we were able to detect certain characteristics that distinguish these isolates from our laboratory $M$. tuberculosis strains and other isolates obtained from the State of Tennessee strain depository which did not produce PGL (section 5.2.3.3). Such results demonstrate the value of using such global technique which allows the simultaneous monitoring of multiple metabolites in studying variabilities in strain virulence.

\subsection{Future Development}

The research discussed in this dissertation represents the first steps towards the development of a novel "omics" approach designed specifically for the analysis of the mycobacterial cell wall metabolites. Also, it can be used for the investigation of other bacterial metabolites as long as these bacteria can grow in the laboratory in well defined growth media. Like other metabolomics methods, the extent to which our proposed methodology can be applied will depend on result-validation and reproducibility as well as user convenience. Convenience does not only include simplicity of the procedures but extends also to data analysis and data sharing. Based on our knowledge of previous experiences in the development of other "omic" approaches, future research efforts for this approach are likely to be directed to each of three main areas.

\subsubsection{Assignment of the 2D-HSQC Signals}

With decades of research directed towards Mycobacteria cell wall lipids, there is no shortage of structural data concerning cell wall lipids. However, the available information may not be extrapolated to the 2D-HSQC lipid profiles due to variation in the data acquisition parameters like the solvent used, acquisition temperature, etc. So before overlaying old data on the 2D-lipid profile, it has to be assured that the acquisition parameters for both spectra can be matched or at least accounted for. In some cases like tracking a specific lipid molecule (as in section 5.2.3.1), it is possible to direct some effort towards isolating this molecule or a close analogue and develop its standardized 2D-HSQC spectrum for subsequent assignment. Also some advanced NMR experiments such as 3D-HCCH -TOCSY can help establish relationships between signals in the 2D spectrum and aid towards establishing a complete assignment.

However, there are also countless reports of lipid species that were isolated from a single specific strain which make handling of such amount of data a challenging task. Cooperative efforts between different research groups that include sharing of data and isolated standards will be necessary to complete signal assignment. A small scale initiative similar to the whole genome sequencing projects may be a good way to enhance and organize efforts in that area. 


\subsubsection{Method Standardization and Data Analysis}

One of the main challenges of metabolomics and lipidomics methods is to develop a valid statistical methodology to insure validity of the data and allow for data comparisons between different research groups. Internal standard has to be added for spectral alignments and at least three replicates are required to validate the reproducibility of the data. Raw data have to be normalized to the internal standard and an average of multiple experiments has to be created. These requirements of multiple experiments and additional processing steps represent a computational challenge especially with the extraordinary complexity of the raw data. Statistical software might be necessary for the comparative analysis between two spectra that may display variability in the background noise or signal intensity. As it is the case with all "omics" techniques, an arithmetic algorithm is needed to represent the data in a bioinformatics-friendly manner. For MS and HPLC methods certain statistical soft wares are now available and some algorithm has been suggested to facilitate data representation.

\subsubsection{Data Depositing and Sharing}

The great advancement in science in the past two decades has resulted in the accumulation of an unprecedented amount of information. For this information to be handled and put to the maximum use by every researcher, bioinformatics science was developed. With the introduction of the World Wide Web, bioinformatics has matured, flourished and become an indispensable tool since then. Bioinformatics offered researchers the possibility of an instant information sharing and rapid effective data search. The ultimate result was an explosion of scientific data which in turn necessitated the creation of organized depository databases and the development of data mining tools to facilitate navigation through the deposited data.

In the lipidomics and metabolomics fields several depository web sites have been established. For example for the "human lipidome" there is several initiative and depository database like LIPIDMAPS for analysis of human lipid pool and biosynthetic pathways. For maximum utility of any new lipidomics technique, the data generated from the technique has to be deposited in an organized database that allows researchers to navigate the information. Such availability of information can encourage researchers to use the technique and apply this methodology in their own investigation and eventually deposit more data. Organized data sharing quite often deters duplication of efforts and speeds up the process of knowledge gain towards the goal of improving our understanding of the world around us.

\subsection{How Metabolomics May Change Life Science}

Unlike genomics which deals primarily with macromolecules and utilize molecular biology tools, metabolomics deals with small organic molecules and utilizes primarily analytical chemistry tools. Currently, major ideas for the development of lipidomics 
techniques are falling in the foot steps of the proteomics science. Although this may be considered as a jump start for lipidomics, the different chemical nature of lipids, as opposed to proteins, will eventually led us to explore new territories in analytical chemistry. Comprehensive metabolomics are by nature hybrid biological/chemical experimentation. For example, to investigate the role of certain gene in regulation of cell metabolism, this gene has to be knocked out first and the elimination of the gene function has to be validated by the absence of its direct gene products (most likely a cellular protein ) and its final product (usually a small molecular weight metabolite). Hence the ultimate goal of establishing a relationship between a certain gene and a certain metabolite requires knowledge of molecular biology, organic chemistry, analytical chemistry as well as biostatistics. Such experiments are usually achieved through a team of scientists from these different science fields but it remains essential that each member of the team has a basic level of knowledge in all these disciplines to facilitate the process of communication and flow of data.

For maximum utility of these metabolomics experiments, a generation of scientists is needed who are trained to develop a thorough understanding of organic and analytical chemistry and cellular and molecular biology as well. These scientists should be regarded as the connecting bridges between chemists and molecular biologists who have been trapped by the lack of communication for so many years because they don't understand each other languages. This lack of communication is a result of the pressure on scientists to develop and adhere to specific specialties and subspecialties because for so long we held specialists in much higher regards. Not all scientists have to abandon the specialist culture but at least enough of them should abandon it in order to build bridges between multiple disciplines. These scientists should also be held in high regards as they contribute to the common good of our human society. 


\section{LIST OF REFERENCES}

1. Goodfellow, M. \& Magee, J. Taxonomy of mycobacteria. In Mycobacteria, Basic Aspects (eds. Gangadharam, P. \& Jenkins, P.) 3-72 (Springer, New York, 1998).

2. Goslee, S. \& Wolinsky, E. Water as a source of potentially pathogenic mycobacteria. Am Rev Respir Dis 113, 287-92 (1976).

3. Collins, C.H., Grange, J.M. \& Yates, M.D. Mycobacteria in water. J Appl Bacteriol 57, 193-211 (1984).

4. Wolinsky, E. Nontuberculous mycobacteria and associated diseases. Am Rev Respir Dis 119, 107-59 (1979).

5. Cangelosi, G., Curtiss, J., Behr, M., Bull, T. \& Stinear, T. Biology of waterborne pathogenic mycobacteria. In Pathogenic Mycobacteria in Water (eds. Bartram, J., Cotruvo, A., Dufour, G., Rees, G. \& Pedley, S.) 39-54 (IWA Publishing, London, UK, 2004).

6. Collins, C.H., Grange, J.M. \& Yates, M.D. Unusual opportunist mycobacteria. Med Lab Sci 43, 262-8 (1986).

7. Barksdale, L. \& Kim, K.S. Mycobacterium. Bacteriol Rev 41, 217-372 (1977).

8. Yegian, D. \& Vanderlinde, R.J. The Nature of acid-fastness. J Bacteriol 54, 77783 (1947).

9. Fisher, C.A. \& Barksdale, L. Cytochemical reactions of human leprosy bacilli and mycobacteria: ultrastructural implications. J Bacteriol 113, 1389-99 (1973).

10. Bhatt, A. et al. Deletion of kasB in Mycobacterium tuberculosis causes loss of acid-fastness and subclinical latent tuberculosis in immunocompetent mice. Proc Natl Acad Sci U S A 104, 5157-62 (2007).

11. Stahl, D.A. \& Urbance, J.W. The division between fast and slow-growing species corresponds to natural relationships among the mycobacteria. J Bacteriol 172, 116-24 (1990).

12. WHO. Global tuberculosis control surveillance, planning, financing. 1-20 (World Health Organization, Geneva, Switzerland, 2008).

13. Swift, S. \& Cohen, H. Granulomas of the skin due to Mycobacterium balnei after abrasions from a fish tank. $N$ Engl J Med 267, 1244-6 (1962).

14. van der Werf, T.S. et al. Mycobacterium ulcerans disease. Bull World Health Organ 83, 785-91 (2005). 
15. Jones, D. \& Havlir, D.V. Nontuberculous mycobacteria in the HIV infected patient. Clin Chest Med 23, 665-74 (2002).

16. Davidson, P. M. avium complex and other mycobacteria causing human disease. In Tuberculosis: a Comprehensive International Approach (eds. Reichman, L. \& Hershfield, E.) 324-56 (Marcel Dekker, New York, 1993).

17. Young, L.S. Mycobacterium avium complex infection. J Infect Dis 157, 863-7 (1988).

18. Smith, M.B., Schnadig, V.J., Boyars, M.C. \& Woods, G.L. Clinical and pathologic features of Mycobacterium fortuitum infections. An emerging pathogen in patients with AIDS. Am J Clin Pathol 116, 225-32 (2001).

19. Irgens, L.M. The discovery of Mycobacterium leprae. A medical achievement in the light of evolving scientific methods. Am J Dermatopathol 6, 337-43 (1984).

20. Ayvazian, L. History of tuberculosis. In Tuberculosis: a Comprehensive International Approach (eds. Reichman, L. \& Hershfield, E.) 1-20 (Marcel Dekker, Inc, New York, 1993).

21. Scheindlin, S. The fight against tuberculosis. Mol Interv 6, 124-30 (2006).

22. Nobelprize.org. Nobel prize in physiology or medicine 1905. (Nobel Foundation, Stockholm, Sweden). From http://nobelprize.org/nobel_prizes/medicine/laureates/ 1905/index.html. Accessed on January 9, 2009.

23. Zink, A., Haas, C.J., Reischl, U., Szeimies, U. \& Nerlich, A.G. Molecular analysis of skeletal tuberculosis in an ancient Egyptian population. J Med Microbiol 50, 355-66 (2001).

24. Hershkovitz, I. et al. Detection and molecular characterization of 9,000-year-old Mycobacterium tuberculosis from a Neolithic settlement in the Eastern Mediterranean. PLoS ONE 3, e3426 (2008).

25. MacCallum, P., Tolhurst, J., Buckle, G. \& Sisson, H. A new mycobacterial infection in man. J Pathol Bacteriol 60, 93-122 (1948).

26. Tolhurst, J.C. \& Buckle, G. A new mycobacterial infection in man; experimental investigations in laboratory animals. J Pathol Bacteriol 60, 102-10 (1948).

27. Bloom, B.R. \& Murray, C.J. Tuberculosis: commentary on a reemergent killer. Science 257, 1055-64 (1992).

28. Corbett, E.L. et al. Uptake of workplace HIV counselling and testing: a clusterrandomised trial in Zimbabwe. PLoS Med 3, e238 (2006). 
29. Narain, J.P. \& Lo, Y.R. Epidemiology of HIV-TB in Asia. Indian J Med Res 120, 277-89 (2004).

30. CDC. TB and HIV Coinfection. (Center for Disease Control, Atlanta, Georgia, 2006).

31. Sadoh, W.E. \& Oviawe, O. The economic burden to families of HIV and $\mathrm{HIV} /$ tuberculosis coinfection in a subsidized HIV treatment program. J Natl Med Assoc 99, 627-31 (2007).

32. Zager, E.M. \& McNerney, R. Multidrug-resistant tuberculosis. BMC Infect Dis $\mathbf{8}$, 10 (2008).

33. Dye, C. Doomsday postponed? Preventing and reversing epidemics of drugresistant tuberculosis. Nat Rev Microbiol 7, 81-7 (2009).

34. Dorman, S.E. \& Chaisson, R.E. From magic bullets back to the magic mountain: the rise of extensively drug-resistant tuberculosis. Nat Med 13, 295-8 (2007).

35. Frieden, T.R. et al. The emergence of drug-resistant tuberculosis in New York City. N Engl J Med 328, 521-6 (1993).

36. Gandhi, N.R. et al. Extensively drug-resistant tuberculosis as a cause of death in patients co-infected with tuberculosis and HIV in a rural area of South Africa. Lancet 368, 1575-80 (2006).

37. WHO. Leprosy: global situation. (World Health Organization, Geneva, Switezerland). From http://www.who.int/lep/situation/PrevStart2007a.pdf. Accessed on January 11, 2009.

38. WHO. Buruli ulcer disease: fact sheet. (World Health Organization, Geneva, Switezerland). From http://www.who.int/mediacentre/factsheets/fs199/en/. Accessed on January 11, 2009.

39. Camphausen, R.T., Jones, R.L. \& Brennan, P.J. A glycolipid antigen specific to Mycobacterium paratuberculosis: structure and antigenicity. Proc Natl Acad Sci U $S A$ 82, 3068-72 (1985).

40. Feller, M. et al. Mycobacterium avium subspecies paratuberculosis and Crohn's disease: a systematic review and meta-analysis. Lancet Infect Dis 7, 607-13 (2007).

41. Francis-Floyd, R. \& Yanong, R. Mycobacteriosis in Fish. 1-4 (University of Florida institute of food and agricultural sciences, 1999).

42. Rhodes, M.W. et al. Mycobacterium pseudoshottsii sp. nov., a slowly growing chromogenic species isolated from Chesapeake Bay striped bass (Morone saxatilis). Int J Syst Evol Microbiol 55, 1139-47 (2005). 
43. Kaattari, I.M., Rhodes, M.W., Kaattari, S.L. \& Shotts, E.B. The evolving story of Mycobacterium tuberculosis clade members detected in fish. J Fish Dis 29, 50920 (2006).

44. Baydur, A. The spectrum of extrapulmonary tuberculosis. West J Med 126, 25362 (1977).

45. Aaron, L. et al. Tuberculosis in HIV-infected patients: a comprehensive review. Clin Microbiol Infect 10, 388-98 (2004).

46. Watts, H.G. \& Lifeso, R.M. Tuberculosis of bones and joints. J Bone Joint Surg Am 78, 288-98 (1996).

47. Riley, R. Transmission and enviromental control of tuberculosis. In Tuberculosis: a Comprehensive International Approach (eds. Reichman, L. \& Hershfield, E.) 121-54 (Marcel Dekker, Inc, New York, 1993).

48. Dannenberg, A.M., Jr. Immune mechanisms in the pathogenesis of pulmonary tuberculosis. Rev Infect Dis 11 Suppl 2, S369-78 (1989).

49. Nardell, E. Pathogenesis of tuberculosis. In Tuberculosis: a Comprehensive International Approach (eds. Reichman, L. \& Hershfield, E.) 23-65 (Marcel Dekker, Inc, New York, 1993).

50. Jozefowski, S., Sobota, A. \& Kwiatkowska, K. How Mycobacterium tuberculosis subverts host immune responses. Bioessays 30, 943-54 (2008).

51. Russell, D.G. Who puts the tubercle in tuberculosis? Nat Rev Microbiol 5, 39-47 (2007).

52. Boshoff, H.I. \& Barry, C.E., 3rd. Tuberculosis - metabolism and respiration in the absence of growth. Nat Rev Microbiol 3, 70-80 (2005).

53. Stewart, G.R., Robertson, B.D. \& Young, D.B. Tuberculosis: a problem with persistence. Nat Rev Microbiol 1, 97-105 (2003).

54. Frieden, T.R., Sterling, T.R., Munsiff, S.S., Watt, C.J. \& Dye, C. Tuberculosis. Lancet 362, 887-99 (2003).

55. O'Brien, R. The treatment of tuberculosis. In Tuberculosis: a Comprehensive International Approach (eds. Reichman, L. \& Hershfield, E.) 207-26 (Marcel Dekker, Inc, New York, 1993).

56. Aziz, M.A. et al. Epidemiology of antituberculosis drug resistance (the Global Project on Anti-tuberculosis Drug Resistance Surveillance): an updated analysis. Lancet 368, 2142-54 (2006). 
57. Warner, D.F. \& Mizrahi, V. The survival kit of Mycobacterium tuberculosis. Nat Med 13, 282-4 (2007).

58. Schleifer, K.H. \& Kandler, O. Peptidoglycan types of bacterial cell walls and their taxonomic implications. Bacteriol Rev 36, $407-77$ (1972).

59. Brennan, P.J. \& Nikaido, H. The envelope of mycobacteria. Annu Rev Biochem 64, 29-63 (1995).

60. Brennan, P.J. Structure, function, and biogenesis of the cell wall of Mycobacterium tuberculosis. Tuberculosis (Edinb) 83, 91-7 (2003).

61. Sarma, G.R., Chandramouli, V. \& Venkitasubramanian, T.A. Occurrence of phosphonolipids in mycobacteria. Biochim Biophys Acta 218, 561-3 (1970).

62. Okuyama, H., Kankura, T. \& Nojima, S. Positional distribution of fatty acids in phospholipids from Mycobacteria. J Biochem 61, $732-7$ (1967).

63. Goren, M.B. \& Brennan, P.J. Mycobacterial lipids: chemistry and biological activities. In Tuberculosis (ed. Youmans, G.) 56-135 (Saunders Company, Philadelphia, PA, 1979).

64. Riley, L.W. Of mice, men, and elephants: Mycobacterium tuberculosis cell envelope lipids and pathogenesis. J Clin Invest 116, 1475-8 (2006).

65. Lee, Y.C. \& Ballou, C.E. Structural studies on the myo-inositol mannodides from the glycolipids of Mycobacterium tuberculosis and Mycobacterium phlei. J Biol Chem 239, 1316-27 (1964).

66. Gilleron, M., Quesniaux, V.F. \& Puzo, G. Acylation state of the phosphatidylinositol hexamannosides from Mycobacterium bovis bacillus Calmette Guerin and mycobacterium tuberculosis $\mathrm{H} 37 \mathrm{Rv}$ and its implication in Toll-like receptor response. J Biol Chem 278, 29880-9 (2003).

67. Kordulakova, J. et al. Identification of the required acyltransferase step in the biosynthesis of the phosphatidylinositol mannosides of mycobacterium species. $J$ Biol Chem 278, 36285-95 (2003).

68. Ballou, C.E., Vilkas, E. \& Lederer, E. Structural studies on the myo-inositol phospholipids of Mycobacterium tuberculosis (var. bovis, strain BCG). J Biol Chem 238, 69-76 (1963).

69. Lee, Y.C. \& Ballou, C.E. Complete structures of the glycophospholipids of mycobacteria. Biochemistry 4, 1395-404 (1965).

70. Fischer, K. et al. Mycobacterial phosphatidylinositol mannoside is a natural antigen for CD1d-restricted T cells. Proc Natl Acad Sci U S A 101, 10685-90 (2004). 
71. Sieling, P.A. et al. CD1-restricted T cell recognition of microbial lipoglycan antigens. Science 269, 227-30 (1995).

72. Ernst, W.A. et al. Molecular interaction of $\mathrm{CD} 1 \mathrm{~b}$ with lipoglycan antigens. Immunity 8, 331-40 (1998).

73. Hett, E.C. \& Rubin, E.J. Bacterial growth and cell division: a mycobacterial perspective. Microbiol Mol Biol Rev 72, 126-56 (2008).

74. Mahapatra, S., Crick, D.C., McNeil, M.R. \& Brennan, P.J. Unique structural features of the peptidoglycan of Mycobacterium leprae. J Bacteriol 190, 655-61 (2008).

75. Mahapatra, S. et al. Mycobacterial lipid II is composed of a complex mixture of modified muramyl and peptide moieties linked to decaprenyl phosphate. $J$ Bacteriol 187, 2747-57 (2005).

76. Wietzerbin, J. et al. Occurrence of D-alanyl-(D)-meso-diaminopimelic acid and meso-diaminopimelyl-meso-diaminopimelic acid interpeptide linkages in the peptidoglycan of Mycobacteria. Biochemistry 13, 3471-6 (1974).

77. Draper, P., Kandler, O. \& Darbre, A. Peptidoglycan and arabinogalactan of Mycobacterium leprae. J Gen Microbiol 133, 1187-94 (1987).

78. Crick, D.C., Mahapatra, S. \& Brennan, P.J. Biosynthesis of the arabinogalactanpeptidoglycan complex of Mycobacterium tuberculosis. Glycobiology 11, 107R18R (2001).

79. McNeil, M., Daffe, M. \& Brennan, P.J. Evidence for the nature of the link between the arabinogalactan and peptidoglycan of mycobacterial cell walls. $J$ Biol Chem 265, 18200-6 (1990).

80. Azuma, I., Kimura, H., Niinaka, T., Aoki, T. \& Yamamura, Y. The isolation and purification of polysaccharides from culture filtrate of human tubercle bacilli strain H37Rv. Jpn J Microbiol 12, 367-9 (1968).

81. Daffe, M., Brennan, P.J. \& McNeil, M. Predominant structural features of the cell wall arabinogalactan of Mycobacterium tuberculosis as revealed through characterization of oligoglycosyl alditol fragments by gas chromatography/mass spectrometry and by $1 \mathrm{H}$ and 13C NMR analyses. $J$ Biol Chem 265, 6734-43 (1990).

82. Alderwick, L.J. et al. Deletion of Cg-emb in corynebacterianeae leads to a novel truncated cell wall arabinogalactan, whereas inactivation of $\mathrm{Cg}$-ubiA results in an arabinan-deficient mutant with a cell wall galactan core. J Biol Chem 280, 32362$71(2005)$. 
83. Alderwick, L.J. et al. Arabinan-deficient mutants of Corynebacterium glutamicum and the consequent flux in decaprenylmonophosphoryl-D-arabinose metabolism. Glycobiology 16, 1073-81 (2006).

84. Bhamidi, S. et al. The identification and location of succinyl residues and the characterization of the interior arabinan region allow for a model of the complete primary structure of Mycobacterium tuberculosis mycolyl arabinogalactan. J Biol Chem 283, 12992-3000 (2008).

85. Asselineau, J. \& Lederer, E. Structure of the mycolic acids of Mycobacteria. Nature 166, 782-3 (1950).

86. Minnikin, D.E., Alshamaony, L. \& Goodfellow, M. Differentiation of Mycobacterium, Nocardia, and related taxa by thin-layer chromatographic analysis of whole-organism methanolysates. J Gen Microbiol 88, 200-4 (1975).

87. Collins, M.D., Goodfellow, M. \& Minnikin, D.E. A survey of the structures of mycolic acids in Corynebacterium and related taxa. J Gen Microbiol 128, 129-49 (1982).

88. Kaneda, K. et al. Structure and molecular species composition of three homologous series of alpha-mycolic acids from Mycobacterium spp. J Gen Microbiol 134, 2213-29 (1988).

89. Kaneda, K. et al. Determination of molecular species composition of C80 or longer-chain alpha-mycolic acids in Mycobacterium spp. by gas chromatographymass spectrometry and mass chromatography. J Clin Microbiol 24, 1060-70 (1986).

90. Watanabe, M., Aoyagi, Y., Ridell, M. \& Minnikin, D.E. Separation and characterization of individual mycolic acids in representative mycobacteria. Microbiology 147, 1825-37 (2001).

91. Barry, C.E., 3rd et al. Mycolic acids: structure, biosynthesis and physiological functions. Prog Lipid Res 37, 143-79 (1998).

92. Paul, T.R. \& Beveridge, T.J. Reevaluation of envelope profiles and cytoplasmic ultrastructure of mycobacteria processed by conventional embedding and freezesubstitution protocols. J Bacteriol 174, 6508-17 (1992).

93. Alshamaony, L., Goodfellow, M., Minnikin, D.E. \& Mordarska, H. Free mycolic acids as criteria in the classification of Gordona and the 'rhodochrous' complex. $J$ Gen Microbiol 92, 183-7 (1976).

94. Butler, W.R., Ahearn, D.G. \& Kilburn, J.O. High-performance liquid chromatography of mycolic acids as a tool in the identification of Corynebacterium, Nocardia, Rhodococcus, and Mycobacterium species. J Clin Microbiol 23, 182-5 (1986). 
95. Hoffmann, C., Leis, A., Niederweis, M., Plitzko, J.M. \& Engelhardt, H. Disclosure of the mycobacterial outer membrane: cryo-electron tomography and vitreous sections reveal the lipid bilayer structure. Proc Natl Acad Sci U S A 105, 3963-7 (2008).

96. Brennan, P.J. \& Goren, M.B. Structural studies on the type-specific antigens and lipids of the Mycobacterium avium. Mycobacterium intracellulare.

Mycobacterium scrofulaceum serocomplex. Mycobacterium intracellulare serotype 9. J Biol Chem 254, 4205-11 (1979).

97. Asselineau, J. \& Laneelle, G. Mycobacterial lipids: a historical perspective. Front Biosci 3, e164-74 (1998).

98. Bekierkunst, A. Acute granulomatous response produced in mice by trehalose6,6-dimycolate. J Bacteriol 96, 958-61 (1968).

99. Rao, V., Gao, F., Chen, B., Jacobs, W.R., Jr. \& Glickman, M.S. Transcyclopropanation of mycolic acids on trehalose dimycolate suppresses Mycobacterium tuberculosis -induced inflammation and virulence. J Clin Invest 116, 1660-7 (2006).

100. Briken, V., Porcelli, S.A., Besra, G.S. \& Kremer, L. Mycobacterial lipoarabinomannan and related lipoglycans: from biogenesis to modulation of the immune response. Mol Microbiol 53, 391-403 (2004).

101. Guerardel, Y. et al. Lipomannan and lipoarabinomannan from a clinical isolate of Mycobacterium kansasii: novel structural features and apoptosis-inducing properties. J Biol Chem 278, 36637-51 (2003).

102. Guerardel, Y. et al. Structural study of lipomannan and lipoarabinomannan from Mycobacterium chelonae. Presence of unusual components with alpha 1,3mannopyranose side chains. J Biol Chem 277, 30635-48 (2002).

103. Tatituri, R.V. et al. Structural characterization of a partially arabinosylated lipoarabinomannan variant isolated from a Corynebacterium glutamicum ubiA mutant. Microbiology 153, 2621-9 (2007).

104. Chatterjee, D., Bozic, C.M., McNeil, M. \& Brennan, P.J. Structural features of the arabinan component of the lipoarabinomannan of Mycobacterium tuberculosis. $J$ Biol Chem 266, 9652-60 (1991).

105. Chatterjee, D., Lowell, K., Rivoire, B., McNeil, M.R. \& Brennan, P.J. Lipoarabinomannan of Mycobacterium tuberculosis. Capping with mannosyl residues in some strains. $J$ Biol Chem 267, 6234-9 (1992).

106. Venisse, A., Berjeaud, J.M., Chaurand, P., Gilleron, M. \& Puzo, G. Structural features of lipoarabinomannan from Mycobacterium bovis BCG. Determination 
of molecular mass by laser desorption mass spectrometry. J Biol Chem 268, 12401-11 (1993).

107. Khoo, K.H., Dell, A., Morris, H.R., Brennan, P.J. \& Chatterjee, D. Inositol phosphate capping of the nonreducing termini of lipoarabinomannan from rapidly growing strains of Mycobacterium. J Biol Chem 270, 12380-9 (1995).

108. Prinzis, S., Chatterjee, D. \& Brennan, P.J. Structure and antigenicity of lipoarabinomannan from Mycobacterium bovis BCG. J Gen Microbiol 139, 264958 (1993).

109. Nigou, J., Zelle-Rieser, C., Gilleron, M., Thurnher, M. \& Puzo, G. Mannosylated lipoarabinomannans inhibit IL-12 production by human dendritic cells: evidence for a negative signal delivered through the mannose receptor. $J$ Immunol 166, 7477-85 (2001).

110. Chua, J., Vergne, I., Master, S. \& Deretic, V. A tale of two lipids: Mycobacterium tuberculosis phagosome maturation arrest. Curr Opin Microbiol 7, $71-7$ (2004).

111. Dao, D.N. et al. Mycobacterium tuberculosis lipomannan induces apoptosis and interleukin-12 production in macrophages. Infect Immun 72, 2067-74 (2004).

112. Vignal, C. et al. Lipomannans, but not lipoarabinomannans, purified from Mycobacterium chelonae and Mycobacterium kansasii induce TNF-alpha and IL8 secretion by a CD14-toll-like receptor 2-dependent mechanism. J Immunol 171, 2014-23 (2003).

113. Guidry, T.V., Hunter, R.L., Jr. \& Actor, J.K. Mycobacterial glycolipid trehalose 6,6'-dimycolate-induced hypersensitive granulomas: contribution of CD4+ lymphocytes. Microbiology 153, 3360-9 (2007).

114. Harland, C.W., Rabuka, D., Bertozzi, C.R. \& Parthasarathy, R. The Mycobacterium tuberculosis virulence factor trehalose dimycolate imparts desiccation resistance to model mycobacterial membranes. Biophys J 94, 4718-24 (2008).

115. Moody, D.B. et al. Structural requirements for glycolipid antigen recognition by CD1b-restricted T cells. Science 278, 283-6 (1997).

116. Daffe, M., Lacave, C., Laneelle, M.A., Gillois, M. \& Laneelle, G. Polyphthienoyl trehalose, glycolipids specific for virulent strains of the tubercle bacillus. Eur $J$ Biochem 172, 579-84 (1988).

117. Lemassu, A., Laneelle, M.A. \& Daffe, M. Revised structure of a trehalosecontaining immunoreactive glycolipid of Mycobacterium tuberculosis. FEMS Microbiol Lett 62, 171-5 (1991). 
118. Gautier, N., Lopez Marin, L.M., Laneelle, M.A. \& Daffe, M. Structure of mycoside $\mathrm{F}$, a family of trehalose-containing glycolipids of Mycobacterium fortuitum. FEMS Microbiol Lett 77, 81-7 (1992).

119. Munoz, M. et al. Occurrence of an antigenic triacyl trehalose in clinical isolates and reference strains of Mycobacterium tuberculosis. FEMS Microbiol Lett 157, 251-9 (1997).

120. Vergne, I. \& Daffe, M. Interaction of mycobacterial glycolipids with host cells. Front Biosci 3, d865-76 (1998).

121. Goren, M.B. Sulfolipid I of Mycobacterium tuberculosis, strain H37Rv. II. Structural studies. Biochim Biophys Acta 210, 127-38 (1970).

122. Goren, M.B. Sulfolipid I of Mycobacterium tuberculosis, strain H37Rv. I. Purification and properties. Biochim Biophys Acta 210, 116-26 (1970).

123. Goren, M.B., Brokl, O. \& Schaefer, W.B. Lipids of putative relevance to virulence in Mycobacterium tuberculosis: correlation of virulence with elaboration of sulfatides and strongly acidic lipids. Infect Immun 9, 142-9 (1974).

124. Middlebrook, G., Coleman, C.M. \& Schaefer, W.B. Sulfolipid from virulent tubercle bacilli. Proc Natl Acad Sci U S A 45, 1801-4 (1959).

125. Goren, M.B., D'Arcy Hart, P., Young, M.R. \& Armstrong, J.A. Prevention of phagosome-lysosome fusion in cultured macrophages by sulfatides of Mycobacterium tuberculosis. Proc Natl Acad Sci U S A 73, 2510-4 (1976).

126. Rousseau, C. et al. Deficiency in mycolipenate- and mycosanoate-derived acyltrehaloses enhances early interactions of Mycobacterium tuberculosis with host cells. Cell Microbiol 5, 405-15 (2003).

127. Rousseau, C. et al. Virulence attenuation of two Mas-like polyketide synthase mutants of Mycobacterium tuberculosis. Microbiology 149, 1837-47 (2003).

128. Hunter, S.W., Murphy, R.C., Clay, K., Goren, M.B. \& Brennan, P.J. Trehalosecontaining lipooligosaccharides. A new class of species-specific antigens from Mycobacterium. J Biol Chem 258, 10481-7 (1983).

129. Saadat, S. \& Ballou, C.E. Pyruvylated glycolipids from Mycobacterium smegmatis. Structures of two oligosaccharide components. J Biol Chem 258, 1813-8 (1983).

130. Hunter, S.W., Fujiwara, T., Murphy, R.C. \& Brennan, P.J. N-acylkansosamine. A novel $\mathrm{N}$-acylamino sugar from the trehalose-containing lipooligosaccharide antigens of Mycobacterium kansasii. J Biol Chem 259, 9729-34 (1984). 
131. Kamisango, K., Saadat, S., Dell, A. \& Ballou, C.E. Pyruvylated glycolipids from Mycobacterium smegmatis. Nature and location of the lipid components. J Biol Chem 260, 4117-21 (1985).

132. Daffe, M., McNeil, M. \& Brennan, P.J. Novel type-specific lipooligosaccharides from Mycobacterium tuberculosis. Biochemistry 30, 378-88 (1991).

133. Besra, G.S. et al. Trehalose-containing lipooligosaccharides of Mycobacterium gordonae: presence of a mono-O-methyltetra-O-acyltrehalose "core" and branching in the oligosaccharide backbone. Biochemistry 32, 12705-14 (1993).

134. Gilleron, M., Vercauteren, J. \& Puzo, G. Lipooligosaccharidic antigen containing a novel C4-branched 3,6-dideoxy-alpha-hexopyranose typifies Mycobacterium gastri. J Biol Chem 268, 3168-79 (1993).

135. Laneelle, M.A., Silve, G., Lopez Marin, L.M. \& Daffe, M. Structures of the glycolipid antigens of members of the third biovariant complex of Mycobacterium fortuitum. Eur J Biochem 238, 270-9 (1996).

136. Ren, H. et al. Identification of the lipooligosaccharide biosynthetic gene cluster from Mycobacterium marinum. Mol Microbiol 63, 1345-59 (2007).

137. Cason, J., Freeman, N.K. \& Sumrell, G. The principal structural features of C27phthienoic acid. J Biol Chem 192, 415-24 (1951).

138. Cason, J. \& Sumrell, G. Investigation of a fraction of acids of the phthioic type from the tubercle bacillus. J Biol Chem 192, 405-13 (1951).

139. Dubey, V.S., Sirakova, T.D. \& Kolattukudy, P.E. Disruption of msl3 abolishes the synthesis of mycolipanoic and mycolipenic acids required for polyacyltrehalose synthesis in Mycobacterium tuberculosis H37Rv and causes cell aggregation. Mol Microbiol 45, 1451-9 (2002).

140. Minnikin, D.E., Kremer, L., Dover, L.G. \& Besra, G.S. The methyl-branched fortifications of Mycobacterium tuberculosis. Chem Biol 9, 545-53 (2002).

141. Goren, M.B., Brokl, O., Das, B.C. \& Lederer, E. Sulfolipid I of Mycobacterium tuberculosis, strain H37RV. Nature of the acyl substituents. Biochemistry 10, 7281 (1971).

142. Sirakova, T.D., Thirumala, A.K., Dubey, V.S., Sprecher, H. \& Kolattukudy, P.E. The Mycobacterium tuberculosis pks2 gene encodes the synthase for the heptaand octamethyl-branched fatty acids required for sulfolipid synthesis. J Biol Chem 276, 16833-9 (2001).

143. Cho, S.N., Yanagihara, D.L., Hunter, S.W., Gelber, R.H. \& Brennan, P.J. Serological specificity of phenolic glycolipid I from Mycobacterium leprae and use in serodiagnosis of leprosy. Infect Immun 41, 1077-83 (1983). 
144. Hunter, S.W. \& Brennan, P.J. Further specific extracellular phenolic glycolipid antigens and a related diacylphthiocerol from Mycobacterium leprae. $J$ Biol Chem 258, 7556-62 (1983).

145. Daffe, M. \& Laneelle, M.A. Distribution of phthiocerol diester, phenolic mycosides and related compounds in mycobacteria. J Gen Microbiol 134, 204955 (1988).

146. Goren, M.B., Brokl, O. \& Schaefer, W.B. Lipids of putative relevance to virulence in Mycobacterium tuberculosis: phthiocerol dimycocerosate and the attenuation indicator lipid. Infect Immun 9, 150-8 (1974).

147. Malaga, W. et al. Deciphering the genetic bases of the structural diversity of phenolic glycolipids in strains of the Mycobacterium tuberculosis complex. J Biol Chem 283, 15177-84 (2008).

148. Cruaud, P., Papa, F., David, H.L. \& Daffe, M. Specificity and antigenicity of mycoside $\mathrm{G}$ and other glycolipids from Mycobacterium marinum. Acta Leprol 7 Suppl 1, 94-7 (1989).

149. Daffe, M., Papa, F., Laszlo, A. \& David, H.L. Glycolipids of recent clinical isolates of Mycobacterium tuberculosis: chemical characterization and immunoreactivity. J Gen Microbiol 135, 2759-66 (1989).

150. Constant, P. et al. Role of the pks $15 / 1$ gene in the biosynthesis of phenolglycolipids in the Mycobacterium tuberculosis complex. Evidence that all strains synthesize glycosylated p-hydroxybenzoic methyl esters and that strains devoid of phenolglycolipids harbor a frameshift mutation in the pks15/1 gene. $J$ Biol Chem 277, 38148-58 (2002).

151. Perez, E. et al. Molecular dissection of the role of two methyltransferases in the biosynthesis of phenolglycolipids and phthiocerol dimycoserosate in the Mycobacterium tuberculosis complex. J Biol Chem 279, 42584-92 (2004).

152. Perez, E. et al. Characterization of three glycosyltransferases involved in the biosynthesis of the phenolic glycolipid antigens from the Mycobacterium tuberculosis complex. J Biol Chem 279, 42574-83 (2004).

153. Reed, M.B. et al. A glycolipid of hypervirulent tuberculosis strains that inhibits the innate immune response. Nature 431, 84-7 (2004).

154. Smith, D.W., Randall, H.M., Maclennan, A.P. \& Lederer, E. Mycosides: a new class of type-specific glycolipids of Mycobacteria. Nature 186, 887-8 (1960).

155. Chatterjee, D. \& Khoo, K.H. The surface glycopeptidolipids of mycobacteria: structures and biological properties. Cell Mol Life Sci 58, 2018-42 (2001). 
156. Laneelle, G. \& Asselineau, J. [Structure of a peptidolipid glycoside isolated from a Mycobacterium]. Eur J Biochem 5, 487-91 (1968).

157. Belisle, J.T., McNeil, M.R., Chatterjee, D., Inamine, J.M. \& Brennan, P.J. Expression of the core lipopeptide of the glycopeptidolipid surface antigens in rough mutants of Mycobacterium avium. J Biol Chem 268, 10510-6 (1993).

158. Antoine, A.D. \& Tepper, B.S. Environmental control of glycogen and lipid content of Mycobacterium tuberculosis. J Bacteriol 100, 538-9 (1969).

159. Brennan, P.J., Rooney, S.A. \& Winder, F.G. The lipids of Mycobacterium tuberculosis BCG: fractionation, composition, turnover and the effects of isoniazid. Ir J Med Sci 3, 371-90 (1970).

160. Winder, F.G., Tighe, J.J. \& Brennan, P.J. Turnover of acylglucose, acyltrehalose and free trehalose during growth of Mycobacterium smegmatis on glucose. J Gen Microbiol 73, 539-46 (1972).

161. Walker, R.W., Barakat, H. \& Hung, J.G. The positional distribution of fatty acids in the phospholipids and triglycerides of Mycobacterium smegmatis and M. bovis BCG. Lipids 5, 684-91 (1970).

162. Snow, G.A. Mycobactins: iron-chelating growth factors from mycobacteria. Bacteriol Rev 34, 99-125 (1970).

163. Snow, G.A. \& White, A.J. Chemical and biological properties of mycobactins isolated from various mycobacteria. Biochem J 115, 1031-50 (1969).

164. Ratledge, C. Iron, mycobacteria and tuberculosis. Tuberculosis (Edinb) 84, 11030 (2004).

165. Barry, C.E., 3rd \& Boshoff, H. Getting the iron out. Nat Chem Biol 1, 127-8 (2005).

166. Greatbanks, D. \& Bedford, G.R. Identification of mycobactins by nuclearmagnetic-resonance spectroscopy. Biochem J 115, 1047-50 (1969).

167. Barclay, R., Ewing, D.F. \& Ratledge, C. Isolation, identification, and structural analysis of the mycobactins of Mycobacterium avium, Mycobacterium intracellulare, Mycobacterium scrofulaceum, and Mycobacterium paratuberculosis. J Bacteriol 164, 896-903 (1985).

168. Ratledge, C. \& Ewing, M. The occurrence of carboxymycobactin, the siderophore of pathogenic mycobacteria, as a second extracellular siderophore in Mycobacterium smegmatis. Microbiology 142 (Pt 8), 2207-12 (1996). 
169. Gobin, J., Wong, D.K., Gibson, B.W. \& Horwitz, M.A. Characterization of exochelins of the Mycobacterium bovis type strain and BCG substrains. Infect Immun 67, 2035-9 (1999).

170. Baker, J.A. Light as a factor in the production of pigment by certain bacteria. $J$ Bacteriol 35, 625-31 (1938).

171. David, H.L. Response of Mycobacteria to ultraviolet light radiation. Am Rev Respir Dis 108, 1175-85 (1973).

172. Collins, M.D., Pirouz, T., Goodfellow, M. \& Minnikin, D.E. Distribution of menaquinones in actinomycetes and corynebacteria. J Gen Microbiol 100, 221-30 (1977).

173. Collins, M.D., Goodfellow, M., Minnikin, D.E. \& Alderson, G. Menaquinone composition of mycolic acid-containing actinomycetes and some sporoactinomycetes. J Appl Bacteriol 58, 77-86 (1985).

174. Sinsimer, D. et al. The phenolic glycolipid of Mycobacterium tuberculosis differentially modulates the early host cytokine response but does not in itself confer hypervirulence. Infect Immun 76, 3027-36 (2008).

175. Indrigo, J., Hunter, R.L., Jr. \& Actor, J.K. Cord factor trehalose 6,6'-dimycolate (TDM) mediates trafficking events during mycobacterial infection of murine macrophages. Microbiology 149, 2049-59 (2003).

176. Villeneuve, C. et al. Surface-exposed glycopeptidolipids of Mycobacterium smegmatis specifically inhibit the phagocytosis of mycobacteria by human macrophages. Identification of a novel family of glycopeptidolipids. J Biol Chem 278, 51291-300 (2003).

177. Pabst, M.J., Gross, J.M., Brozna, J.P. \& Goren, M.B. Inhibition of macrophage priming by sulfatide from Mycobacterium tuberculosis. J Immunol 140, 634-40 (1988).

178. Seya, T. et al. Two receptor theory in innate immune activation: studies on the receptors for bacillus Culmet Guillen-cell wall skeleton. Arch Immunol Ther Exp (Warsz) 49 Suppl 1, S13-21 (2001).

179. Tsuboi, A. et al. Enhanced induction of human WT1-specific cytotoxic T lymphocytes with a 9-mer WT1 peptide modified at HLA-A*2402-binding residues. Cancer Immunol Immunother 51, 614-20 (2002).

180. Yoo, Y.C., Hata, K., Lee, K.B. \& Azuma, I. Inhibitory effect of BCG cell-wall skeletons (BCG-CWS) emulsified in squalane on tumor growth and metastasis in mice. Arch Pharm Res 25, $522-7$ (2002). 
181. Geisel, R.E., Sakamoto, K., Russell, D.G. \& Rhoades, E.R. In vivo activity of released cell wall lipids of Mycobacterium bovis bacillus Calmette-Guerin is due principally to trehalose mycolates. J Immunol 174, 5007-15 (2005).

182. Rhoades, E.R., Geisel, R.E., Butcher, B.A., McDonough, S. \& Russell, D.G. Cell wall lipids from Mycobacterium bovis BCG are inflammatory when inoculated within a gel matrix: characterization of a new model of the granulomatous response to mycobacterial components. Tuberculosis (Edinb) 85, 159-76 (2005).

183. Andersen, C.S. et al. A simple mycobacterial monomycolated glycerol lipid has potent immunostimulatory activity. $J$ Immunol 182, 424-32 (2009).

184. O'Donnell, A., Minnikin, D.E. \& Goodfellow, M. Integrated lipid and wall analysis of Actinomycetes. In Chemical Methods in Bacterial Systematics (eds. Goodfellow, M. \& Minnikin, D.E) 131-43 (Academic Press, London, 1985).

185. Minnikin, D.E., Parlett, J.H., Magnusson, M., Ridell, M. \& Lind, A. Mycolic acid patterns of representatives of Mycobacterium bovis BCG. J Gen Microbiol 130, 2733-6 (1984).

186. Yuan, Y. \& Barry, C.E., 3rd. A common mechanism for the biosynthesis of methoxy and cyclopropyl mycolic acids in Mycobacterium tuberculosis. Proc Natl Acad Sci US A 93, 12828-33 (1996).

187. Butler, W., Floyd, M. \& Silcox, V. Standardized Method for HPLC Identification of Mycobacteria. 3-14 (Center for Disease Control and Prevention, Atlanta, GA, 1996).

188. Phiet, P.H., Wietzerbin, J., Zissman, E., Petit, J.F. \& Lederer, E. Analysis of the cell wall of five strains of Myocbacterium tuberculosis BCG and of an attenuated human strain, W 115. Infect Immun 13, 677-81 (1976).

189. Lee, R.E., Li, W., Chatterjee, D. \& Lee, R.E. Rapid structural characterization of the arabinogalactan and lipoarabinomannan in live mycobacterial cells using $2 \mathrm{D}$ and 3D HR-MAS NMR: structural changes in the arabinan due to ethambutol treatment and gene mutation are observed. Glycobiology 15, 139-51 (2005).

190. Li, W., Lee, R.E., Lee, R.E. \& Li, J. Methods for acquisition and assignment of multidimensional high-resolution magic angle spinning NMR of whole cell bacteria. Anal Chem 77, 5785-92 (2005).

191. Folch, J., Lees, M. \& Sloane Stanley, G.H. A simple method for the isolation and purification of total lipides from animal tissues. J Biol Chem 226, 497-509 (1957).

192. Bligh, E.G. \& Dyer, W.J. A rapid method of total lipid extraction and purification. Can J Biochem Physiol 37, 911-7 (1959). 
193. Larsson, L. \& Mardh, P.A. Gas chromatographic characterization of mycobacteria: analysis of fatty acids and trifluoroacetylated whole-cell methanolysates. J Clin Microbiol 3, 81-5 (1976).

194. Tisdall, P.A., Roberts, G.D. \& Anhalt, J.P. Identification of clinical isolates of mycobacteria with gas-liquid chromatography alone. J Clin Microbiol 10, 506-14 (1979).

195. Minnikin, D.E. \& Goodfellow, M. Lipid composition in the classification and identification of acid-fast bacteria. Soc Appl Bacteriol Symp Ser 8, 189-256 (1980).

196. Knisley, C.V., Damato, J.J., McClatchy, J.K. \& Brennan, P.J. Rapid and sensitive identification of Mycobacterium tuberculosis. J Clin Microbiol 22, 761-7 (1985).

197. Levy-Frebault, V., Goh, K.S. \& David, H.L. Mycolic acid analysis for clinical identification of Mycobacterium avium and related mycobacteria. $J$ Clin Microbiol 24, 835-9 (1986).

198. Stover, C.K. et al. A small-molecule nitroimidazopyran drug candidate for the treatment of tuberculosis. Nature 405, 962-6 (2000).

199. Uenishi, Y., Fujita, Y., Kusunose, N., Yano, I. \& Sunagawa, M. Comprehensive analysis of mycolic acid subclass and molecular species composition of Mycobacterium bovis BCG Tokyo 172 cell wall skeleton (SMP-105). J Microbiol Methods 72, 149-56 (2008).

200. Dobson, G. et al. Systematic analysis of complex mycobacterial lipids. In Chemical Methods in Bacterial Systematics (eds. Goodfellow, M. \& Minnikin, D.E.) 237-65 (Academic Press, London, 1985).

201. Reiner, E., Beam, R.E. \& Kubica, G.P. Pyrolysis-gas-liquid chromatography studies for the classification of mycobacteria. Am Rev Respir Dis 99, 750-9 (1969).

202. Reiner, E., Hicks, J.J., Ball, M.M. \& Martin, W.J. Rapid characterization of salmonella organisms by means of pyrolysis-gas-liquid chromatography. Anal Chem 44, 1058-61 (1972).

203. Reiner, E., Hicks, J.J., Beam, R.E. \& David, H.L. Recent studies on mycobacterial differentiation by means of pyrolysis-gas-liquid chromatography. Am Rev Respir Dis 104, 656-60 (1971).

204. Lambert, M.A., Moss, C.W., Silcox, V.A. \& Good, R.C. Analysis of mycolic acid cleavage products and cellular fatty acids of Mycobacterium species by capillary gas chromatography. J Clin Microbiol 23, 731-6 (1986). 
205. Mosca, A., Russo, F., Miragliotta, L., Iodice, M.A. \& Miragliotta, G. Utility of gas chromatography for rapid identification of mycobacterial species frequently encountered in clinical laboratory. J Microbiol Methods 68, 392-5 (2007).

206. Smid, I. \& Salfinger, M. Mycobacterial identification by computer-aided gasliquid chromatography. Diagn Microbiol Infect Dis 19, 81-8 (1994).

207. Butler, W.R., Jost, K.C., Jr. \& Kilburn, J.O. Identification of mycobacteria by high-performance liquid chromatography. J Clin Microbiol 29, 2468-72 (1991).

208. Butler, W.R. \& Kilburn, J.O. High-performance liquid chromatography patterns of mycolic acids as criteria for identification of Mycobacterium chelonae, Mycobacterium fortuitum, and Mycobacterium smegmatis. J Clin Microbiol 28, 2094-8 (1990).

209. Thibert, L. \& Lapierre, S. Routine application of high-performance liquid chromatography for identification of mycobacteria. J Clin Microbiol 31, 1759-63 (1993).

210. Kellogg, J.A. et al. Application of the Sherlock Mycobacteria Identification System using high-performance liquid chromatography in a clinical laboratory. $J$ Clin Microbiol 39, 964-70 (2001).

211. Viader-Salvado, J.M., Molina-Torres, C.A. \& Guerrero-Olazaran, M. Detection and identification of mycobacteria by mycolic acid analysis of sputum specimens and young cultures. $J$ Microbiol Methods 70, 479-83 (2007).

212. Parrish, N. et al. Rapid, standardized method for determination of Mycobacterium tuberculosis drug susceptibility by use of mycolic acid analysis. J Clin Microbiol 45, 3915-20 (2007).

213. Besra, G.S. et al. Further structural definition of a new family of glycopeptidolipids from Mycobacterium xenopi. Biochemistry 32, 347-55 (1993).

214. Khoo, K.H. et al. Structural definition of the glycopeptidolipids and the pyruvylated, glycosylated acyltrehalose from Mycobacterium butyricum. Carbohydr Res 276, 449-55 (1995).

215. Jain, M. et al. Lipidomics reveals control of Mycobacterium tuberculosis virulence lipids via metabolic coupling. Proc Natl Acad Sci U S A 104, 5133-8 (2007).

216. Shui, G., Bendt, A.K., Pethe, K., Dick, T. \& Wenk, M.R. Sensitive profiling of chemically diverse bioactive lipids. J Lipid Res 48, 1976-84 (2007).

217. Gomez, J.E. \& McKinney, J.D. M. tuberculosis persistence, latency, and drug tolerance. Tuberculosis (Edinb) 84, 29-44 (2004). 
218. Manabe, Y.C. \& Bishai, W.R. Latent Mycobacterium tuberculosis-persistence, patience, and winning by waiting. Nat Med 6, 1327-9 (2000).

219. Karakousis, P.C., Bishai, W.R. \& Dorman, S.E. Mycobacterium tuberculosis cell envelope lipids and the host immune response. Cell Microbiol 6, 105-16 (2004).

220. Xie, Z., Siddiqi, N. \& Rubin, E.J. Differential antibiotic susceptibilities of starved Mycobacterium tuberculosis isolates. Antimicrob Agents Chemother 49, 4778-80 (2005).

221. Cole, S.T. et al. Deciphering the biology of Mycobacterium tuberculosis from the complete genome sequence. Nature 393, 537-44 (1998).

222. Brosch, R. et al. Genomic analysis reveals variation between Mycobacterium tuberculosis $\mathrm{H} 37 \mathrm{Rv}$ and the attenuated M. tuberculosis H37Ra strain. Infect Immun 67, 5768-74 (1999).

223. Manabe, Y.C., Dannenberg, A.M., Jr. \& Bishai, W.R. What we can learn from the Mycobacterium tuberculosis genome sequencing projects. Int J Tuberc Lung Dis 4, S18-23 (2000).

224. Fleischmann, R.D. et al. Whole-genome comparison of Mycobacterium tuberculosis clinical and laboratory strains. J Bacteriol 184, 5479-90 (2002).

225. Gruft, H., Falkinham, J.O., 3rd \& Parker, B.C. Recent experience in the epidemiology of disease caused by atypical mycobacteria. Rev Infect Dis 3, 990-6 (1981).

226. Horsburgh, C.R., Jr. \& Selik, R.M. The epidemiology of disseminated nontuberculous mycobacterial infection in the acquired immunodeficiency syndrome (AIDS). Am Rev Respir Dis 139, 4-7 (1989).

227. Glassroth, J. Pulmonary disease due to nontuberculous mycobacteria. Chest 133, 243-51 (2008).

228. Oren, B., Raz, R. \& Hass, H. Urinary Mycobacterium fortuitum infection. Infection 18, 105-6 (1990).

229. Shitrit, D. et al. Pulmonary Mycobacterium kansasii infection in Israel, 19992004: clinical features, drug susceptibility, and outcome. Chest 129, 771-6 (2006).

230. Serra, C., Loi, G., Saddi, B., Pautasso, M. \& Manzin, A. Unusual clinical presentation of Mycobacterium fortuitum infection in an immunocompetent woman. J Clin Microbiol 45, 1663-5 (2007).

231. George, K.M. et al. Mycolactone: a polyketide toxin from Mycobacterium ulcerans required for virulence. Science 283, 854-7 (1999). 
232. Mahrous, E.A., Lee, R.E. \& Lee, R.E. Lipid profiling using two dimensional heteronuclear single quantum coherence NMR. In Lipidomics: Methods and Protocols (Humana Press, New York, 2009).In press.

233. Lechevalier, H.A., Lechevalier, M.P. \& Gerber, N.N. Chemical composition as a criterion in the classification of actinomycetes. Adv Appl Microbiol 14, 47-72 (1971).

234. Lechevalier, M.P., Horan, A.C. \& Lechevalier, H. Lipid composition in the classification of nocardiae and mycobacteria. $J$ Bacteriol 105, 313-8 (1971).

235. Brennan, P.J., Lehane, D.P. \& Thomas, D.W. Acylglucoses of the corynebacteria and mycobacteria. Eur J Biochem 13, 117-23 (1970).

236. Wenk, M.R. The emerging field of lipidomics. Nat Rev Drug Discov 4, 594-610 (2005).

237. Bhatt, K., Gurcha, S.S., Bhatt, A., Besra, G.S. \& Jacobs, W.R., Jr. Two polyketide-synthase-associated acyltransferases are required for sulfolipid biosynthesis in Mycobacterium tuberculosis. Microbiology 153, 513-20 (2007).

238. Villeneuve, C. et al. Mycobacteria use their surface-exposed glycolipids to infect human macrophages through a receptor-dependent process. J Lipid Res $\mathbf{4 6}$, 47583 (2005).

239. Watve, M.G., Tickoo, R., Jog, M.M. \& Bhole, B.D. How many antibiotics are produced by the genus Streptomyces? Arch Microbiol 176, 386-90 (2001).

240. Mahrous, E.A., Lee, R.E. \& Lee, R.E. A rapid approach to lipid profiling of mycobacteria using 2D HSQC NMR maps. J Lipid Res 49, 455-63 (2008).

241. Cosma, C.L., Sherman, D.R. \& Ramakrishnan, L. The secret lives of the pathogenic mycobacteria. Annu Rev Microbiol 57, $641-76$ (2003).

242. Faber, W.R. Life-threatening mycobacteria infection. Clin Dermatol 23, 254-7 (2005).

243. Gooding, T.M. et al. Immune response to infection with Mycobacterium ulcerans. Infect Immun 69, 1704-7 (2001).

244. van der Werf, T.S., van der Graaf, W.T., Tappero, J.W. \& Asiedu, K. Mycobacterium ulcerans infection. Lancet 354, 1013-8 (1999).

245. Buckle, G. \& Tolhurst, J.C. A new mycobacterial infection in man; cultivation of the new Mycobacterial infection in man; cultivation of the new Mycobacterium. $J$ Pathol Bacteriol 60, 116-22 (1948). 
246. Collins, C.H., Grange, J.M., Noble, W.C. \& Yates, M.D. Mycobacterium marinum infections in man. J Hyg (Lond) 94, 135-49 (1985).

247. Wayne, L.G. \& Sramek, H.A. Agents of newly recognized or infrequently encountered mycobacterial diseases. Clin Microbiol Rev 5, 1-25 (1992).

248. Kim, R.D. et al. Pulmonary nontuberculous mycobacterial disease: prospective study of a distinct preexisting syndrome. Am J Respir Crit Care Med 178, 106674 (2008).

249. Miguez-Burbano, M.J. et al. Non-tuberculous mycobacteria disease as a cause of hospitalization in HIV-infected subjects. Int J Infect Dis 10, 47-55 (2006).

250. Thoen, C.O. Mycobacterial infections other than tuberculosis. In The Merck Veterinary Manual. (Merck and Co. Inc, New jersey, USA, 2008). From http://www.merckvetmanual.com/mvm/index.jsp?cfile=htm/bc/52300.htm. Accessed on January 23, 2009.

251. Yamada, Y., Inouye, G., Tahara, Y. \& Kondo, K. The structure of the menaquinones with a tetrahydrogenated isoprenoid side-chain. Biochim Biophys Acta 488, 280-4 (1977).

252. Lemassu, A. et al. Extracellular and surface-exposed polysaccharides of nontuberculous mycobacteria. Microbiology 142 (Pt 6), 1513-20 (1996).

253. Nigou, J., Gilleron, M. \& Puzo, G. Lipoarabinomannans: from structure to biosynthesis. Biochimie 85, 153-66 (2003).

254. Mve-Obiang, A. et al. A newly discovered mycobacterial pathogen isolated from laboratory colonies of Xenopus species with lethal infections produces a novel form of mycolactone, the Mycobacterium ulcerans macrolide toxin. Infect Immun 73, 3307-12 (2005).

255. Trott, K.A. et al. Characterization of a Mycobacterium ulcerans-like infection in a colony of African tropical clawed frogs (Xenopus tropicalis). Comp Med 54, 30917 (2004).

256. Stinear, T.P. et al. Reductive evolution and niche adaptation inferred from the genome of Mycobacterium ulcerans, the causative agent of Buruli ulcer. Genome Res 17, 192-200 (2007).

257. Stinear, T.P. et al. Insights from the complete genome sequence of Mycobacterium marinum on the evolution of Mycobacterium tuberculosis. Genome Res 18, 729-41 (2008).

258. Yip, M.J. et al. Evolution of Mycobacterium ulcerans and other mycolactoneproducing mycobacteria from a common Mycobacterium marinum progenitor. $J$ Bacteriol 189, 2021-9 (2007). 
259. Daffe, M., Varnerot, A. \& Levy-Frebault, V.V. The phenolic mycoside of Mycobacterium ulcerans: structure and taxonomic implications. J Gen Microbiol 138, 131-7 (1992).

260. Riviere, M., Fournie, J.J. \& Puzo, G. A novel mannose containing phenolic glycolipid from Mycobacterium kansasii. J Biol Chem 262, 14879-84 (1987).

261. Chatterjee, D., Bozic, C.M., Knisley, C., Cho, S.N. \& Brennan, P.J. Phenolic glycolipids of Mycobacterium bovis: new structures and synthesis of a corresponding seroreactive neoglycoprotein. Infect Immun 57, 322-30 (1989).

262. Dobson, G., Minnikin, D.E., Besra, G.S., Mallet, A.I. \& Magnusson, M. Characterisation of phenolic glycolipids from Mycobacterium marinum. Biochim Biophys Acta 1042, 176-81 (1990).

263. Reed, M.B., Gagneux, S., Deriemer, K., Small, P.M. \& Barry, C.E., 3rd. The WBeijing lineage of Mycobacterium tuberculosis overproduces triglycerides and has the DosR dormancy regulon constitutively upregulated. J Bacteriol 189, 2583-9 (2007).

264. Puech, V. et al. Structure of the cell envelope of corynebacteria: importance of the non-covalently bound lipids in the formation of the cell wall permeability barrier and fracture plane. Microbiology 147, 1365-82 (2001).

265. Fischer, W., Laine, R.A. \& Nakano, M. On the relationship between glycerophosphoglycolipids and lipoteichoic acids in Gram-positive bacteria. II. Structures of glycerophosphoglycolipids. Biochim Biophys Acta 528, 298-308 (1978).

266. Li, Y. \& Gray, G.R. Structural identification of a major mitogenic lipid derived from Bacillus subtilis as a glycerophosphoglycolipid. Biochemistry 35, 16299-304 (1996).

267. Perkins, J.E. The conquest of tuberculosis. American Journal of Public Health 55, 2049 (1965).

268. Jain, A. \& Dixit, P. Multidrug-resistant to extensively drug resistant tuberculosis: What is next? J Biosci 33, 605-16 (2008).

269. Wayne, L.G. \& Sramek, H.A. Metronidazole is bactericidal to dormant cells of Mycobacterium tuberculosis. Antimicrob Agents Chemother 38, 2054-8 (1994).

270. Singh, R. et al. PA-824 kills nonreplicating Mycobacterium tuberculosis by intracellular NO release. Science 322, 1392-5 (2008).

271. Walsh, G.P. et al. The Philippine cynomolgus monkey (Macaca fasicularis) provides a new nonhuman primate model of tuberculosis that resembles human disease. Nat Med 2, 430-6 (1996). 
272. Fuller, C.L., Flynn, J.L. \& Reinhart, T.A. In situ study of abundant expression of proinflammatory chemokines and cytokines in pulmonary granulomas that develop in cynomolgus macaques experimentally infected with Mycobacterium tuberculosis. Infect Immun 71, 7023-34 (2003).

273. Wayne, L.G. \& Hayes, L.G. An in vitro model for sequential study of shiftdown of Mycobacterium tuberculosis through two stages of nonreplicating persistence. Infect Immun 64, 2062-9 (1996).

274. Wayne, L.G. \& Sohaskey, C.D. Nonreplicating persistence of mycobacterium tuberculosis. Anпи Rev Microbiol 55, 139-63 (2001).

275. Wayne, L.G. \& Hayes, L.G. Nitrate reduction as a marker for hypoxic shiftdown of Mycobacterium tuberculosis. Tuber Lung Dis 79, 127-32 (1998).

276. Boshoff, H.I. et al. The transcriptional responses of Mycobacterium tuberculosis to inhibitors of metabolism: novel insights into drug mechanisms of action. $J$ Biol Chem 279, 40174-84 (2004).

277. McKinney, J.D. et al. Persistence of Mycobacterium tuberculosis in macrophages and mice requires the glyoxylate shunt enzyme isocitrate lyase. Nature 406, 735-8 (2000).

278. Daniel, J. et al. Induction of a novel class of diacylglycerol acyltransferases and triacylglycerol accumulation in Mycobacterium tuberculosis as it goes into a dormancy-like state in culture. J Bacteriol 186, 5017-30 (2004).

279. Betts, J.C., Lukey, P.T., Robb, L.C., McAdam, R.A. \& Duncan, K. Evaluation of a nutrient starvation model of Mycobacterium tuberculosis persistence by gene and protein expression profiling. Mol Microbiol 43, 717-31 (2002).

280. Dahl, J.L. et al. The role of RelMtb-mediated adaptation to stationary phase in long-term persistence of Mycobacterium tuberculosis in mice. Proc Natl Acad Sci US A 100, 10026-31 (2003).

281. Smeulders, M.J., Keer, J., Speight, R.A. \& Williams, H.D. Adaptation of Mycobacterium smegmatis to stationary phase. J Bacteriol 181, 270-83 (1999).

282. Rohde, K.H., Abramovitch, R.B. \& Russell, D.G. Mycobacterium tuberculosis invasion of macrophages: linking bacterial gene expression to environmental cues. Cell Host Microbe 2, 352-64 (2007).

283. Findlay, R.H., King, G.M. \& Watling, L. Efficacy of phospholipid analysis in determining microbial biomass in sediments. Appl Environ Microbiol 55, 2888-93 (1989).

284. Van Veldhoven, P.P. \& Mannaerts, G.P. Inorganic and organic phosphate measurements in the nanomolar range. Anal Biochem 161, 45-8 (1987). 
285. Ranger, B.S. et al. Globally distributed mycobacterial fish pathogens produce a novel plasmid-encoded toxic macrolide, mycolactone F. Infect Immun 74, 603745 (2006).

286. Hong, H., Demangel, C., Pidot, S.J., Leadlay, P.F. \& Stinear, T. Mycolactones: immunosuppressive and cytotoxic polyketides produced by aquatic mycobacteria. Nat Prod Rep 25, 447-54 (2008).

287. Gunawardana, G. et al. Characterization of novel macrolide toxins, mycolactone $\mathrm{A}$ and B, from a human pathogen, Mycobacterium ulcerans $J$ Am Chem Soc 121, 6092-3 (1999).

288. Hong, H. et al. Identification using LC-MSn of co-metabolites in the biosynthesis of the polyketide toxin mycolactone by a clinical isolate of Mycobacterium ulcerans. Chem Commun (Camb), 2822-3 (2003).

289. Daniel, A.K., Lee, R.E., Portaels, F. \& Small, P.L. Analysis of Mycobacterium species for the presence of a macrolide toxin, mycolactone. Infect Immun 72, 12332 (2004).

290. Mve-Obiang, A., Lee, R.E., Portaels, F. \& Small, P.L. Heterogeneity of mycolactones produced by clinical isolates of Mycobacterium ulcerans: implications for virulence. Infect Immun 71, 774-83 (2003).

291. Stinear, T.P. et al. Giant plasmid-encoded polyketide synthases produce the macrolide toxin of Mycobacterium ulcerans. Proc Natl Acad Sci U S A 101, $1345-$ 9 (2004).

292. Pidot, S.J. et al. Deciphering the genetic basis for polyketide variation among mycobacteria producing mycolactones. BMC Genomics 9, 462-77 (2008).

293. Fidanze, S., Song, F., Szlosek-Pinaud, M., Small, P.L. \& Kishi, Y. Complete structure of the mycolactones. $J$ Am Chem Soc 123, 10117-8 (2001).

294. Song, F., Fidanze, S., Benowitz, A.B. \& Kishi, Y. Total synthesis of the mycolactones. Org Lett 4, 647-50 (2002).

295. Judd, T.C., Bischoff, A., Kishi, Y., Adusumilli, S. \& Small, P.L. Structure determination of mycolactone C via total synthesis. Org Lett 6, 4901-4 (2004).

296. Hong, H., Spencer, J.B., Porter, J.L., Leadlay, P.F. \& Stinear, T. A novel mycolactone from a clinical isolate of Mycobacterium ulcerans provides evidence for additional toxin heterogeneity as a result of specific changes in the modular polyketide synthase. Chembiochem 6, 643-8 (2005).

297. Aubry, S. et al. Synthesis and structure of mycolactone E isolated from frog Mycobacterium. Org Lett (2008). Epub ahead of print. 
298. Hong, H., Stinear, T., Skelton, P., Spencer, J.B. \& Leadlay, P.F. Structure elucidation of a novel family of mycolactone toxins from the frog pathogen Mycobacterium sp. MU128FXT by mass spectrometry. Chem Commun (Camb), 4306-8 (2005).

299. Cadapan, L.D. et al. Suspension cultivation of Mycobacterium ulcerans for the production of mycolactones. FEMS Microbiol Lett 205, 385-9 (2001).

300. Tsukamura, M. \& Mikoshiba, H. A new Mycobacterium which caused skin infection. Microbiol Immunol 26, 951-5 (1982).

301. Nakanaga, K. et al. "Mycobacterium ulcerans subsp. shinshuense" isolated from a skin ulcer lesion: identification based on 16S rRNA gene sequencing. J Clin Microbiol 45, 3840-3 (2007).

302. Kim, H.J. \& Kishi, Y. Total synthesis and stereochemistry of mycolactone F. $J$ Am Chem Soc 130, 1842-4 (2008).

303. Vercellone, A. \& Puzo, G. New-found phenolic glycolipids in Mycobacterium bovis BCG. Presence of a diglycosylated glycolipid. J Biol Chem 264, 7447-54 (1989).

304. Watanabe, M., Yamada, Y., Iguchi, K. \& Minnikin, D.E. Structural elucidation of new phenolic glycolipids from Mycobacterium tuberculosis. Biochim Biophys Acta 1210, 174-80 (1994).

305. Simeone, R. et al. Molecular dissection of the biosynthetic relationship between phthiocerol and phthiodiolone dimycocerosates and their critical role in the virulence and permeability of Mycobacterium tuberculosis. Febs J 274, 1957-69 (2007).

306. Barry, C.E., 3rd. Interpreting cell wall 'virulence factors' of Mycobacterium tuberculosis. Trends Microbiol 9, 237-41 (2001).

307. Stefani, M.M. et al. Assessment of anti-PGL-I as a prognostic marker of leprosy reaction. Int J Lepr Other Mycobact Dis 66, 356-64 (1998).

308. Stadthagen, G. et al. p-Hydroxybenzoic acid synthesis in Mycobacterium tuberculosis. J Biol Chem 280, 40699-706 (2005).

309. Schena, M., Shalon, D., Davis, R.W. \& Brown, P.O. Quantitative monitoring of gene expression patterns with a complementary DNA microarray. Science $\mathbf{2 7 0}$, 467-70 (1995).

310. Wasinger, V.C. et al. Progress with gene-product mapping of the Mollicutes: Mycoplasma genitalium. Electrophoresis 16, 1090-4 (1995). 
311. James, P. Protein identification in the post-genome era: the rapid rise of proteomics. Q Rev Biophys 30, 279-331 (1997).

312. Oliver, S.G., Winson, M.K., Kell, D.B. \& Baganz, F. Systematic functional analysis of the yeast genome. Trends Biotechnol 16, 373-8 (1998).

313. Roessner, U. et al. Metabolic profiling allows comprehensive phenotyping of genetically or environmentally modified plant systems. Plant Cell 13, 11-29 (2001).

314. Fiehn, O. Combining genomics, metabolome analysis, and biochemical modelling to understand metabolic networks. Comp Funct Genomics 2, 155-68 (2001).

315. Nicholson, J.K., Lindon, J.C. \& Holmes, E. 'Metabonomics': understanding the metabolic responses of living systems to pathophysiological stimuli via multivariate statistical analysis of biological NMR spectroscopic data. Xenobiotica 29, 1181-9 (1999).

316. Fiehn, O. Metabolomics--the link between genotypes and phenotypes. Plant Mol Biol 48, 155-71 (2002).

317. Cole, S.T. et al. Massive gene decay in the leprosy bacillus. Nature 409, 1007-11 (2001).

318. Betts, J.C. Transcriptomics and proteomics: tools for the identification of novel drug targets and vaccine candidates for tuberculosis. IUBMB Life 53, 239-42 (2002).

319. Betts, J.C. et al. Signature gene expression profiles discriminate between isoniazid-, thiolactomycin-, and triclosan-treated Mycobacterium tuberculosis. Antimicrob Agents Chemother 47, 2903-13 (2003).

320. Munoz-Elias, E.J. \& McKinney, J.D. Mycobacterium tuberculosis isocitrate lyases 1 and 2 are jointly required for in vivo growth and virulence. Nat Med 11, 638-44 (2005).

321. Gibney, M.J. et al. Metabolomics in human nutrition: opportunities and challenges. Am J Clin Nutr 82, 497-503 (2005).

322. Griffin, J.L. Metabolic profiles to define the genome: can we hear the phenotypes? Philos Trans R Soc Lond B Biol Sci 359, 857-71 (2004).

323. Fujita, Y., Naka, T., Doi, T. \& Yano, I. Direct molecular mass determination of trehalose monomycolate from 11 species of mycobacteria by MALDI-TOF mass spectrometry. Microbiology 151, 1443-52 (2005).

324. Pulfer, M. \& Murphy, R.C. Electrospray mass spectrometry of phospholipids. Mass Spectrom Rev 22, 332-64 (2003). 
325. Brugger, B., Erben, G., Sandhoff, R., Wieland, F.T. \& Lehmann, W.D.

Quantitative analysis of biological membrane lipids at the low picomole level by nano-electrospray ionization tandem mass spectrometry. Proc Natl Acad Sci U S A 94, 2339-44 (1997).

326. Fujita, Y., Naka, T., McNeil, M.R. \& Yano, I. Intact molecular characterization of cord factor (trehalose 6,6'-dimycolate) from nine species of mycobacteria by MALDI-TOF mass spectrometry. Microbiology 151, 3403-16 (2005).

327. Ikeda, K., Shimizu, T. \& Taguchi, R. Targeted analysis of ganglioside and sulfatide molecular species by LC/ESI-MS/MS with theoretically expanded multiple reaction monitoring. J Lipid Res 49, 2678-89 (2008).

328. Sommer, U., Herscovitz, H., Welty, F.K. \& Costello, C.E. LC-MS-based method for the qualitative and quantitative analysis of complex lipid mixtures. J Lipid Res 47, 804-14 (2006).

329. Han, X. \& Gross, R.W. Shotgun lipidomics: multidimensional MS analysis of cellular lipidomes. Expert Rev Proteomics 2, 253-64 (2005).

330. Han, X., Yang, K., Cheng, H., Fikes, K.N. \& Gross, R.W. Shotgun lipidomics of phosphoethanolamine-containing lipids in biological samples after one-step in situ derivatization. J Lipid Res 46, 1548-60 (2005).

331. Yang, K., Zhao, Z., Gross, R.W. \& Han, X. Systematic analysis of cholinecontaining phospholipids using multi-dimensional mass spectrometry-based shotgun lipidomics. J Chromatogr B Analyt Technol Biomed Life Sci (2009).

332. Han, X. \& Gross, R.W. Global analyses of cellular lipidomes directly from crude extracts of biological samples by ESI mass spectrometry: a bridge to lipidomics. $J$ Lipid Res 44, 1071-9 (2003).

333. Han, X. \& Gross, R.W. Shotgun lipidomics: electrospray ionization mass spectrometric analysis and quantitation of cellular lipidomes directly from crude extracts of biological samples. Mass Spectrom Rev 24, 367-412 (2005).

334. Han, X. et al. Shotgun lipidomics identifies cardiolipin depletion in diabetic myocardium linking altered substrate utilization with mitochondrial dysfunction. Biochemistry 44, 16684-94 (2005).

335. Ekroos, K., Chernushevich, I.V., Simons, K. \& Shevchenko, A. Quantitative profiling of phospholipids by multiple precursor ion scanning on a hybrid quadrupole time-of-flight mass spectrometer. Anal Chem 74, 941-9 (2002).

336. Ito, S., Nabetani, T., Shinoda, Y., Nagatsuka, Y. \& Hirabayashi, Y. Quantitative analysis of a novel glucosylated phospholipid by liquid chromatography-mass spectrometry. Anal Biochem 376, 252-7 (2008). 
337. Bielawski, J., Szulc, Z.M., Hannun, Y.A. \& Bielawska, A. Simultaneous quantitative analysis of bioactive sphingolipids by high-performance liquid chromatography-tandem mass spectrometry. Methods 39, 82-91 (2006).

338. German, J.B., Roberts, M.A. \& Watkins, S.M. Personal metabolomics as a next generation nutritional assessment. J Nutr 133, 4260-6 (2003).

339. German, J.B., Roberts, M.A. \& Watkins, S.M. Genomics and metabolomics as markers for the interaction of diet and health: lessons from lipids. $J$ Nutr 133, 2078S-2083S (2003).

340. Lin, S., Fischl, A.S., Bi, X. \& Parce, W. Separation of phospholipids in microfluidic chip device: application to high-throughput screening assays for lipid-modifying enzymes. Anal Biochem 314, 97-107 (2003).

341. Gawrisch, K., Eldho, N.V. \& Polozov, I.V. Novel NMR tools to study structure and dynamics of biomembranes. Chem Phys Lipids 116, 135-51 (2002).

342. Gavaghan, C.L., Holmes, E., Lenz, E., Wilson, I.D. \& Nicholson, J.K. An NMRbased metabonomic approach to investigate the biochemical consequences of genetic strain differences: application to the C57BL10J and Alpk:ApfCD mouse. FEBS Lett 484, 169-74 (2000).

343. Nicholson, J.K. \& Wilson, I.D. Opinion: understanding 'global' systems biology: metabonomics and the continuum of metabolism. Nat Rev Drug Discov 2, 668-76 (2003).

344. Lewis, I.A. et al. Method for determining molar concentrations of metabolites in complex solutions from two-dimensional 1H-13C NMR spectra. Anal Chem 79, 9385-90 (2007).

345. Kilburn, J.O. \& Takayama, K. Effects of ethambutol on accumulation and secretion of trehalose mycolates and free mycolic acid in Mycobacterium smegmatis. Antimicrob Agents Chemother 20, 401-4 (1981).

346. Takayama, K., Armstrong, E.L., Kunugi, K.A. \& Kilburn, J.O. Inhibition by ethambutol of mycolic acid transfer into the cell wall of Mycobacterium smegmatis. Antimicrob Agents Chemother 16, 240-2 (1979).

347. Gangadharam, P.R., Harold, F.M. \& Schaefer, W.B. Selective inhibition of nucleic acid synthesis in Mycobacterium tuberculosis by isoniazid. Nature 198, $712-4$ (1963).

348. Takayama, K., Wang, L. \& David, H.L. Effect of isoniazid on the in vivo mycolic acid synthesis, cell growth, and viability of Mycobacterium tuberculosis.

Antimicrob Agents Chemother 2, 29-35 (1972). 
349. Wang, L. \& Takayama, K. Relationship between the uptake of isoniazid and its action on in vivo mycolic acid synthesis in Mycobacterium tuberculosis. Antimicrob Agents Chemother 2, 438-41 (1972).

350. Scheindlin, S. Rare diseases, orphan drugs, and orphaned patients. Mol Interv 6, 186-91 (2006).

351. Takayama, K. \& Kilburn, J.O. Inhibition of synthesis of arabinogalactan by ethambutol in Mycobacterium smegmatis. Antimicrob Agents Chemother 33, 1493-9 (1989).

352. Belanger, A.E. et al. The embAB genes of Mycobacterium avium encode an arabinosyl transferase involved in cell wall arabinan biosynthesis that is the target for the antimycobacterial drug ethambutol. Proc Natl Acad Sci U S A 93, 1191924 (1996).

353. Deng, L. et al. Recognition of multiple effects of ethambutol on metabolism of mycobacterial cell envelope. Antimicrob Agents Chemother 39, 694-701 (1995).

354. Houk, V.N., Baker, J.H., Sorensen, K. \& Kent, D.C. The epidemiology of tuberculosis infection in a closed environment. Arch Environ Health 16, 26-35 (1968).

355. Valway, S.E. et al. An outbreak involving extensive transmission of a virulent strain of Mycobacterium tuberculosis. $N$ Engl J Med 338, 633-9 (1998).

356. Manca, C. et al. Mycobacterium tuberculosis CDC1551 induces a more vigorous host response in vivo and in vitro, but is not more virulent than other clinical isolates. J Immunol 162, 6740-6 (1999).

357. Sreevatsan, S. et al. Restricted structural gene polymorphism in the Mycobacterium tuberculosis complex indicates evolutionarily recent global dissemination. Proc Natl Acad Sci U S A 94, 9869-74 (1997).

358. Manca, C. et al. Virulence of a Mycobacterium tuberculosis clinical isolate in mice is determined by failure to induce Th1 type immunity and is associated with induction of IFN-alpha /beta. Proc Natl Acad Sci U S A 98, 5752-7 (2001).

359. Hershberg, R. et al. High functional diversity in Mycobacterium tuberculosis driven by genetic drift and human demography. PLoS Biol 6, e311 (2008).

360. Gagneux, S. \& Small, P.M. Global phylogeography of Mycobacterium tuberculosis and implications for tuberculosis product development. Lancet Infect Dis 7, 328-37 (2007).

361. Flores, L. et al. Large sequence polymorphisms classify Mycobacterium tuberculosis strains with ancestral spoligotyping patterns. J Clin Microbiol 45, 3393-5 (2007). 


\section{APPENDIX A. SUPPLEMENTARY FIGURES FOR CHAPTER 2}
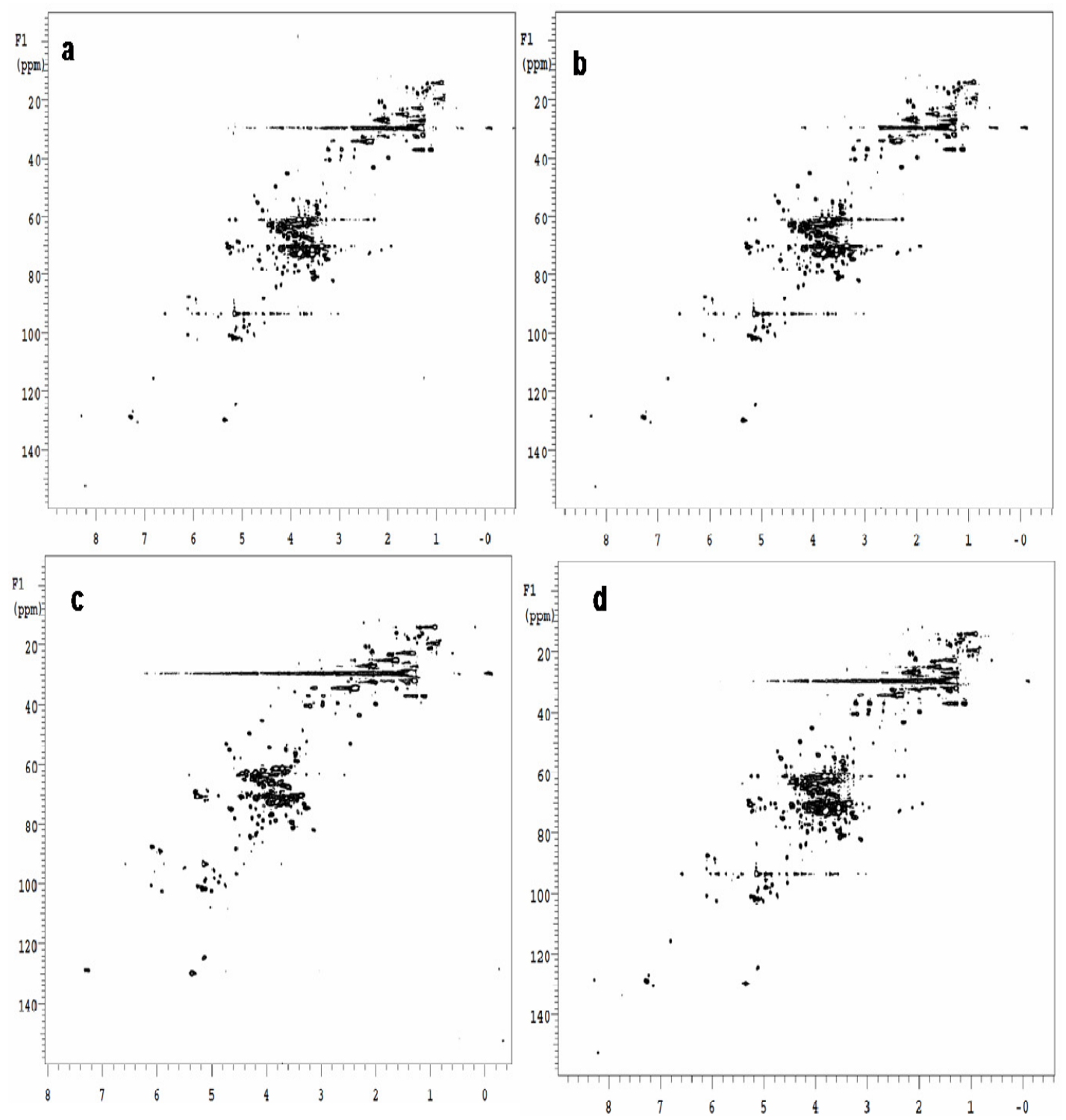

Figure A.1 Effect of Different Acquisition Time on the Quality of the 2D-HSQC Lipid Profiles

2D-HSQC lipid profiles of $M$. smegmatis acquired for (a), 2 transients, 20 minutes, (b), 4 transients, 40 minutes, (c), 12 transients, 120 minutes and, (d), 60 transients, 10 hours. 

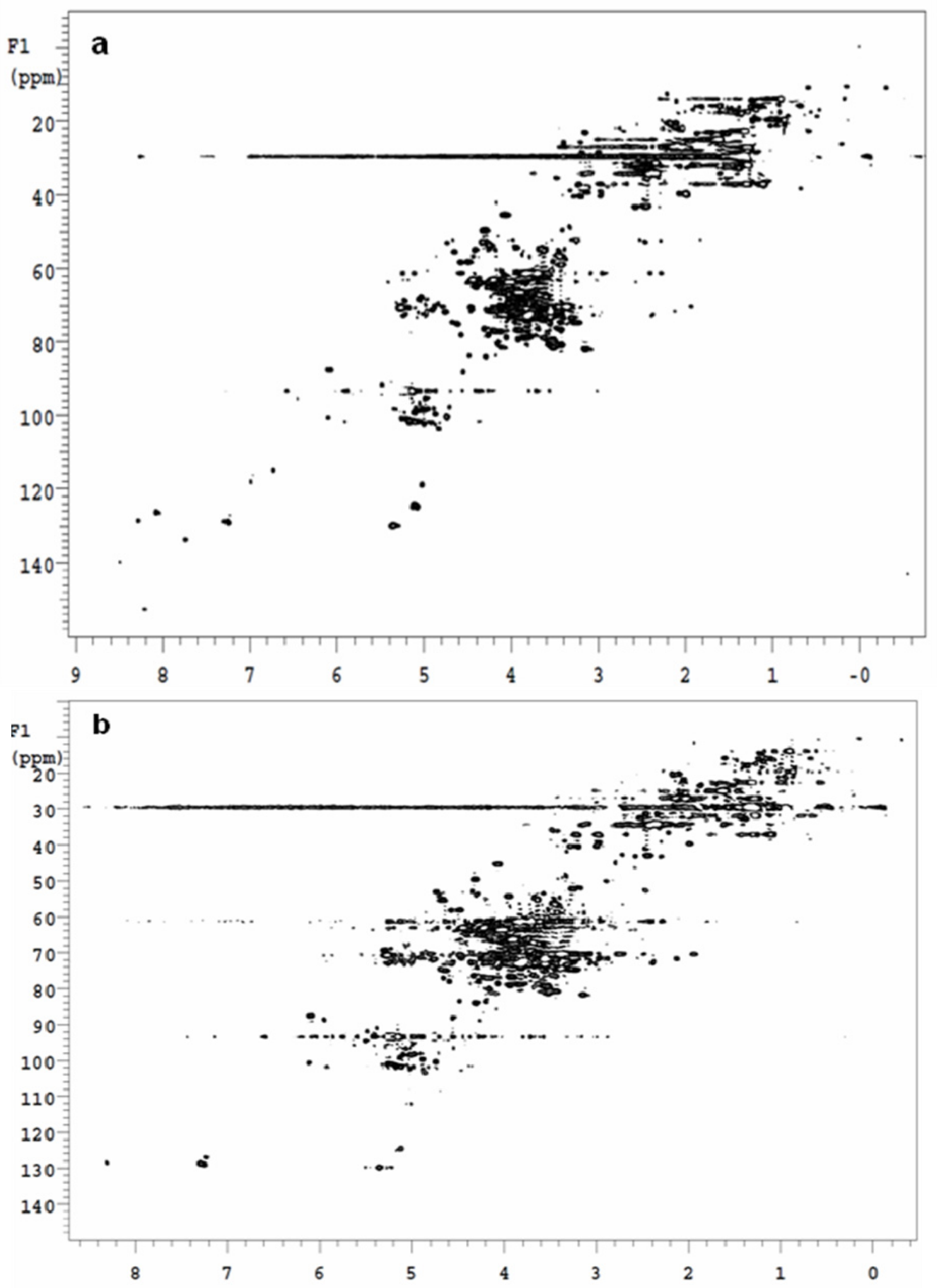

Figure A.2 Effect of Different Growth Conditions (Liquid Media Versus Solid Media)

Lipid profiles of M. avium growing in Middlebrook 7H9, (a), or growing on Middlebrook 7 H11 agar, (b). 


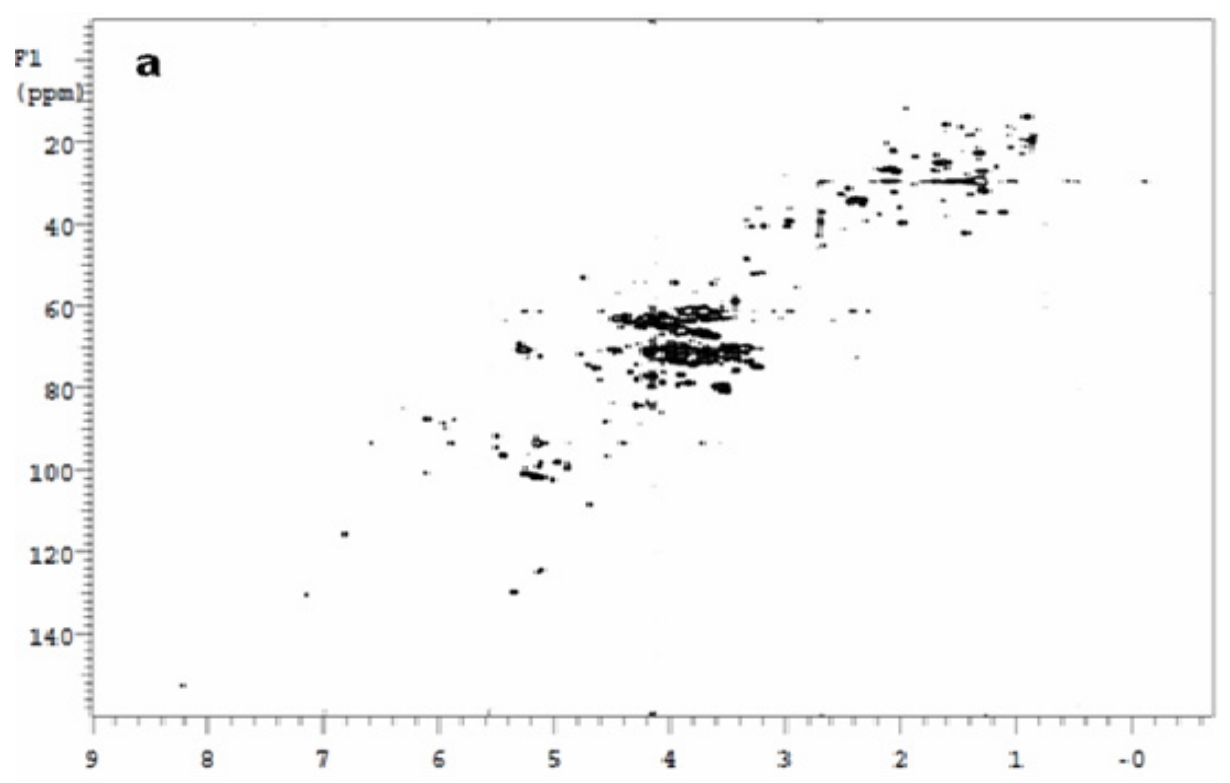

Figure A.3 Lipid Profiles of M. bovis BCG in the Early Exponential Phase Cells were harvested and extracted at O.D ${ }_{\lambda 600}=0.29$.

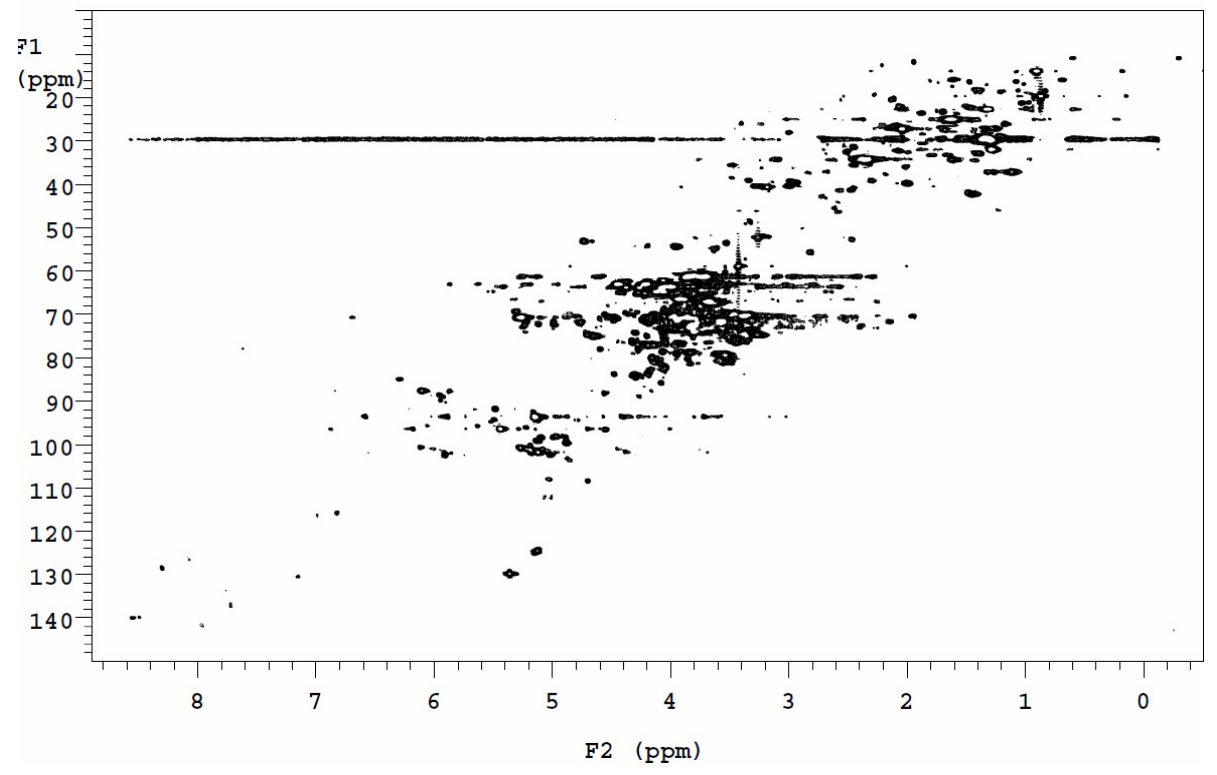

Figure A.4 Lipid Profiles of M. bovis BCG of Cells in the Late Growth Phase Cells were harvested and extracted at O.D $\mathrm{D}_{\lambda 600}=1.2$. 

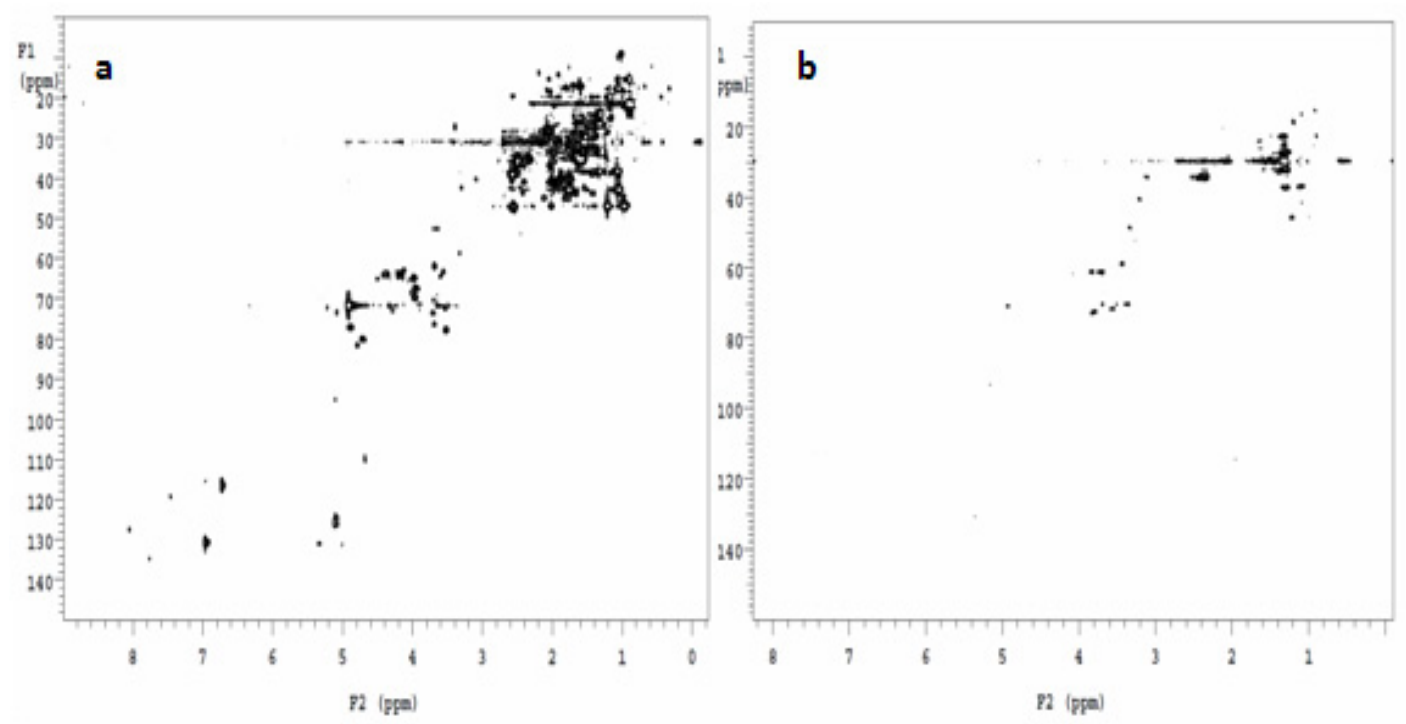

Figure A.5 Effect of ${ }^{13} \mathrm{C}$-acetate Labeling on the Lipid Profile of M. ulcerans

Cells were grown in Middlebrook $7 \mathrm{H} 9$ where ${ }^{13} \mathrm{C}_{6}$-dextrose and ${ }^{13} \mathrm{C}_{3}$-glycerol were used, (a), or in regular Middlebrook $7 \mathrm{H} 9$ where $0.2 \%{ }^{13} \mathrm{C}_{2}$ sodium acetate have been added, (b). 

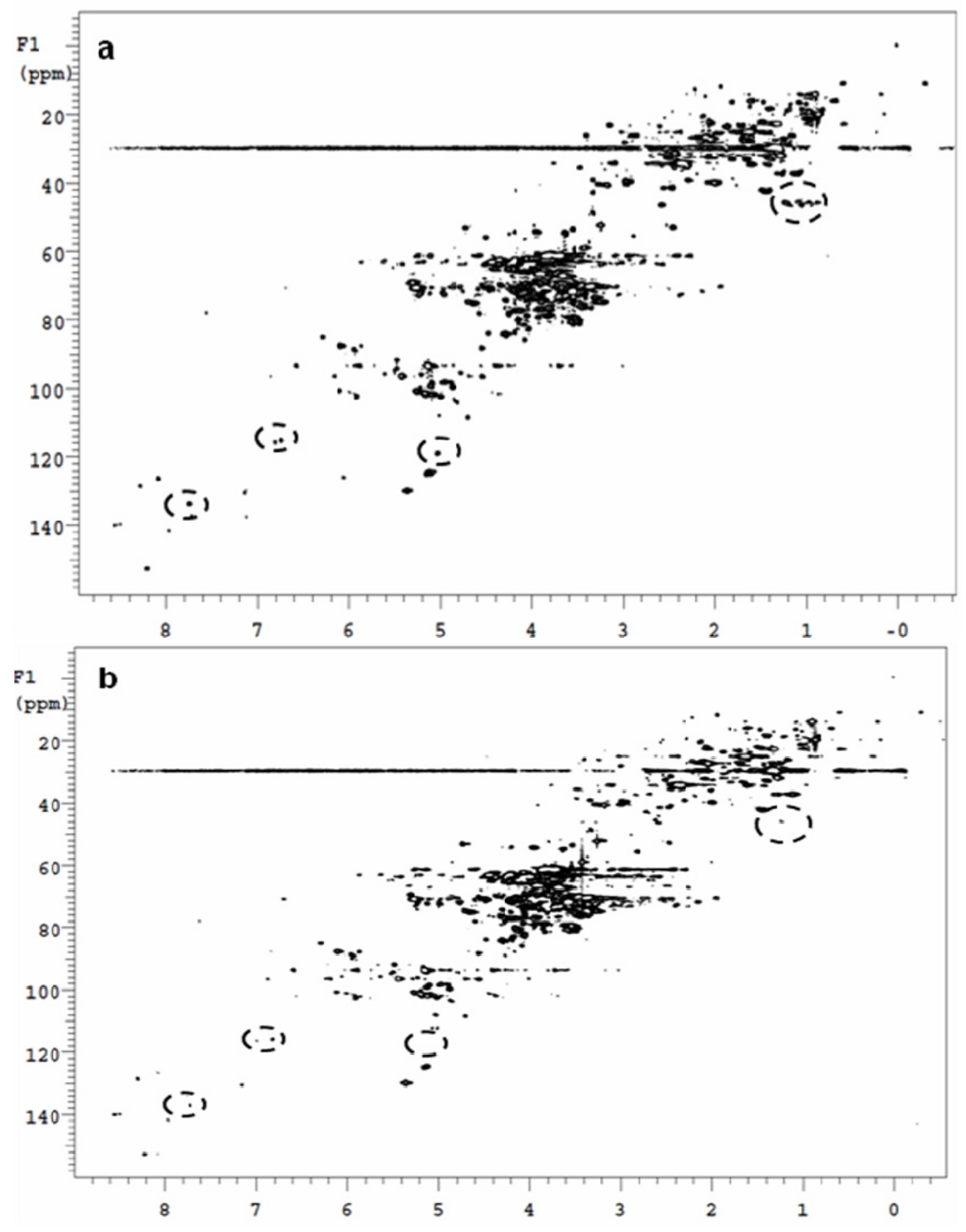

Figure A.6 Effect of Using Different Culture Volumes for Lipid Extraction

M. bovis BCG were harvested and extracted from $20 \mathrm{mls}$ culture, (a), or from $50 \mathrm{mls}$ culture, (b), to a final extract volume of $600 \mu 1$. Both profiles were acquired using $5 \mathrm{~mm}$ trpfg NMR probe. Low abundance lipids were better represented in the first spectrum due to the presence of lower amounts of TAG and PL. 

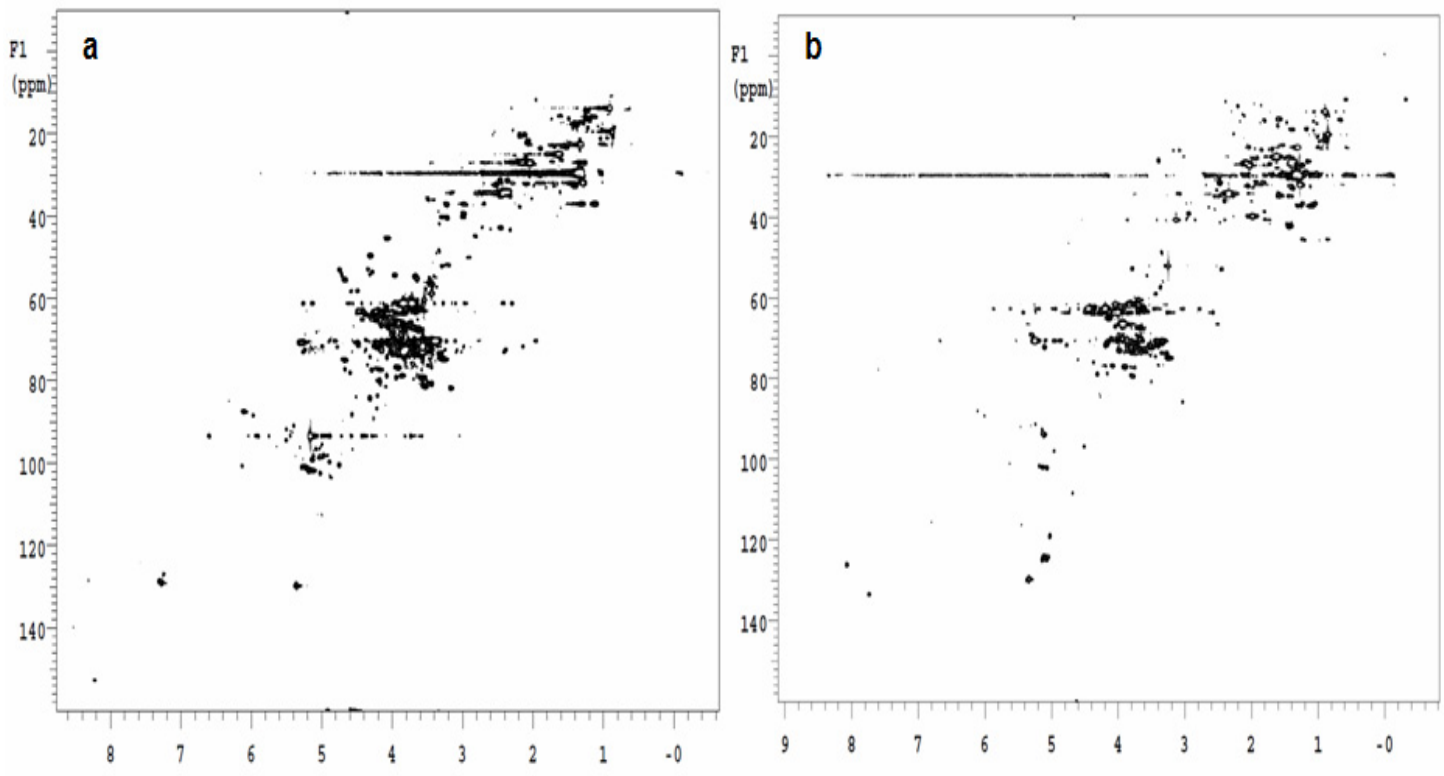

Figure A.7 Lipid Profiles Obtained from the Extraction of 10mls Culture

Lipid profiles were acquired using 3mm IFC NMR probe of $M$. avium, (a), and $M$. tuberculosis, (b). 

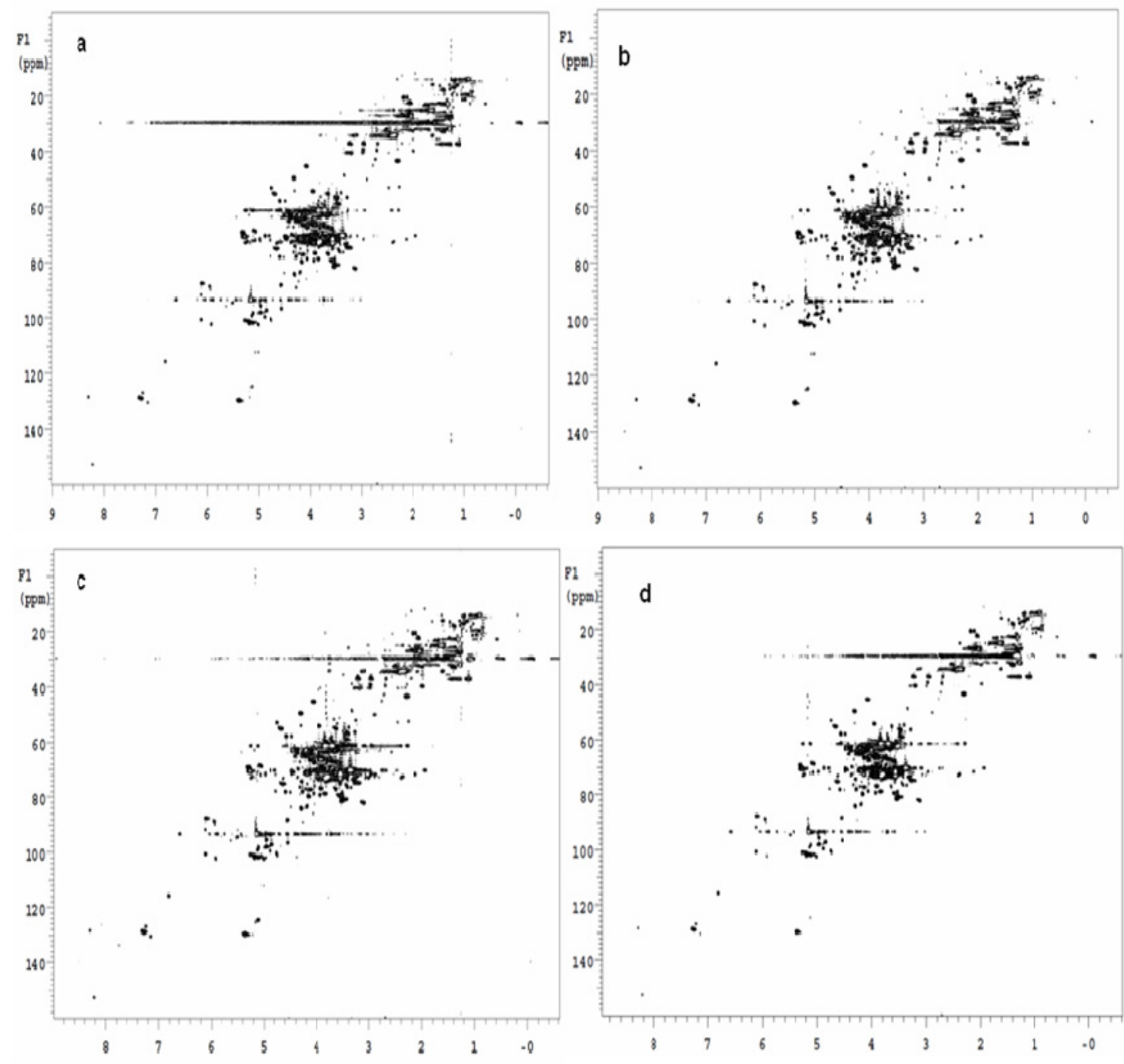

Figure A.8 Effect of Different Extraction Time on the Quality of the Lipid Profiles

M. smegmatis cells were harvested and extracted for 20 minutes, (a), 40 minutes, (b), 90 minutes, (c), and 120 minutes, (d). All profiles were acquired for 40 minutes. 

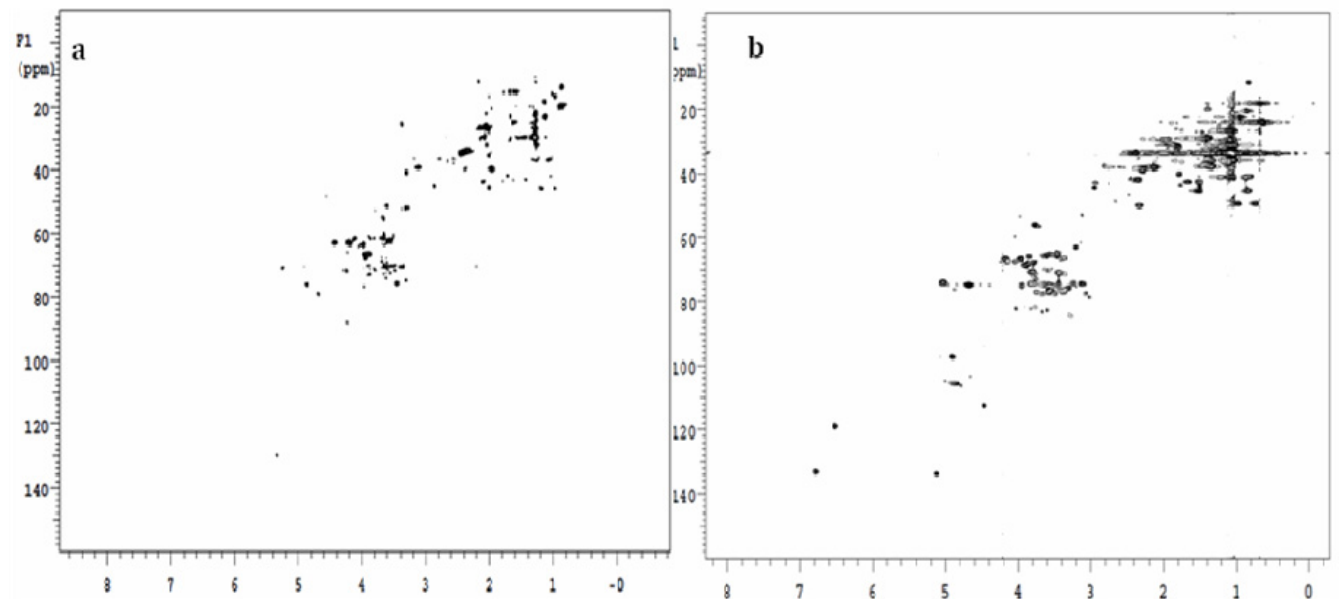

Figure A.9 Lipid Profiles Produced by the Extraction of the Washed Cell Pellet with Acetone- $\mathrm{d}_{6}$

M. ulcerans cells were extracted for 90 minutes with either acetone- $\mathrm{d}_{6}$, (a) or 2:1 mixture of $\mathrm{CDCl}_{3}: \mathrm{CD}_{3} \mathrm{OD}$, (b).

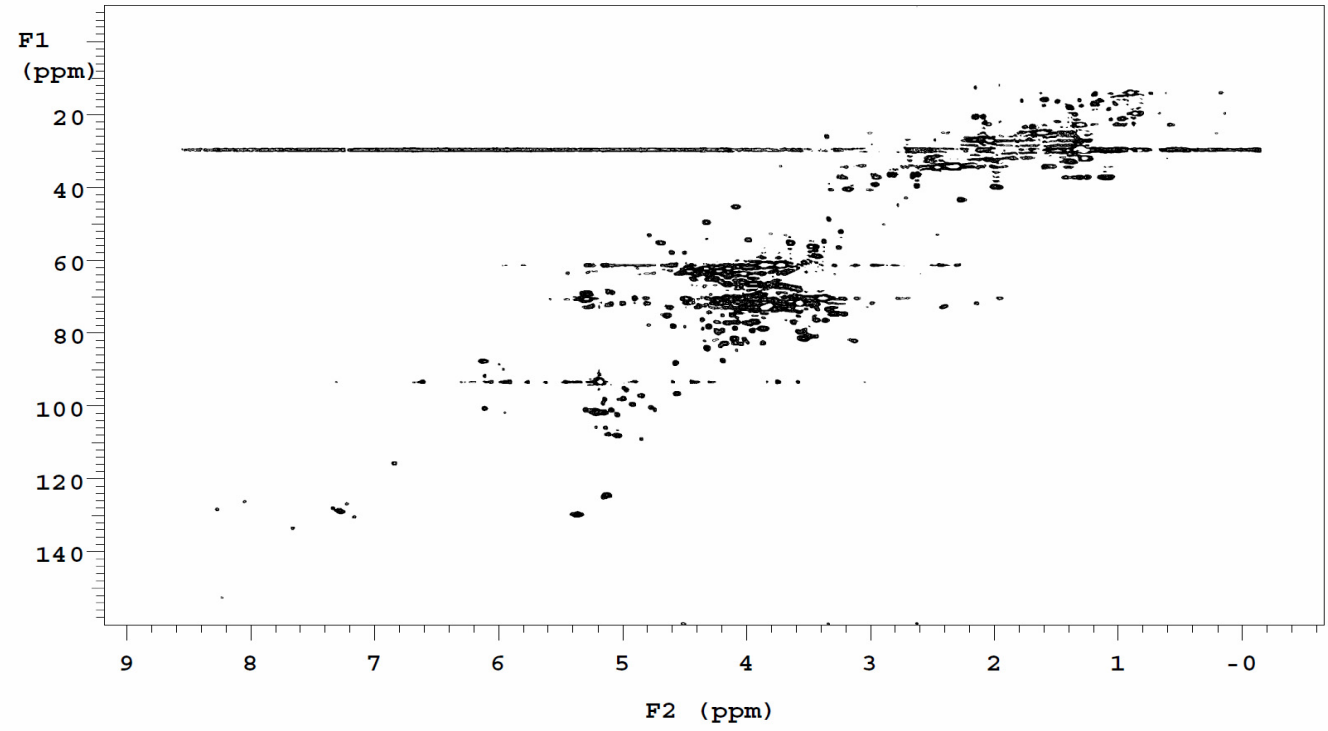

Figure A.10 Effect of Extraction with Benzene- $d_{6}$

Lipid profile of cells extracted with benzene- $\mathrm{d}_{6}$ included only signals of fatty acids. Shown above is the lipid profile obtained when a $2: 1$ mixture of $\mathrm{CDCl}_{3}: \mathrm{CD}_{3} \mathrm{OD}$ was used for re-extraction of the pellet remaining after extraction with benzene. 

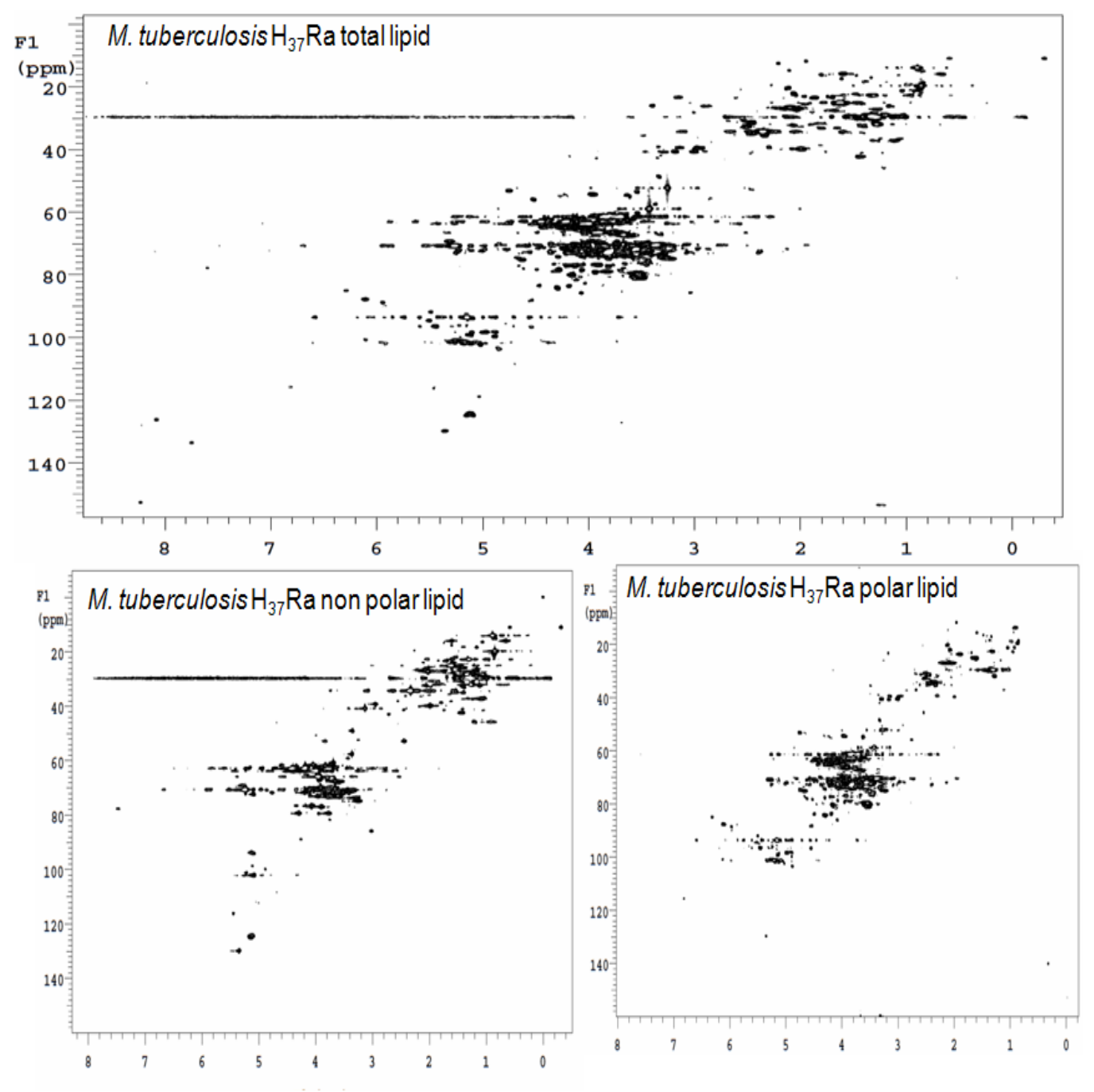

Figure A.11 Effect of Solvent Partition on the Lipid Profiles of M. tuberculosis $\mathrm{H}_{37} \mathrm{Ra}$ 


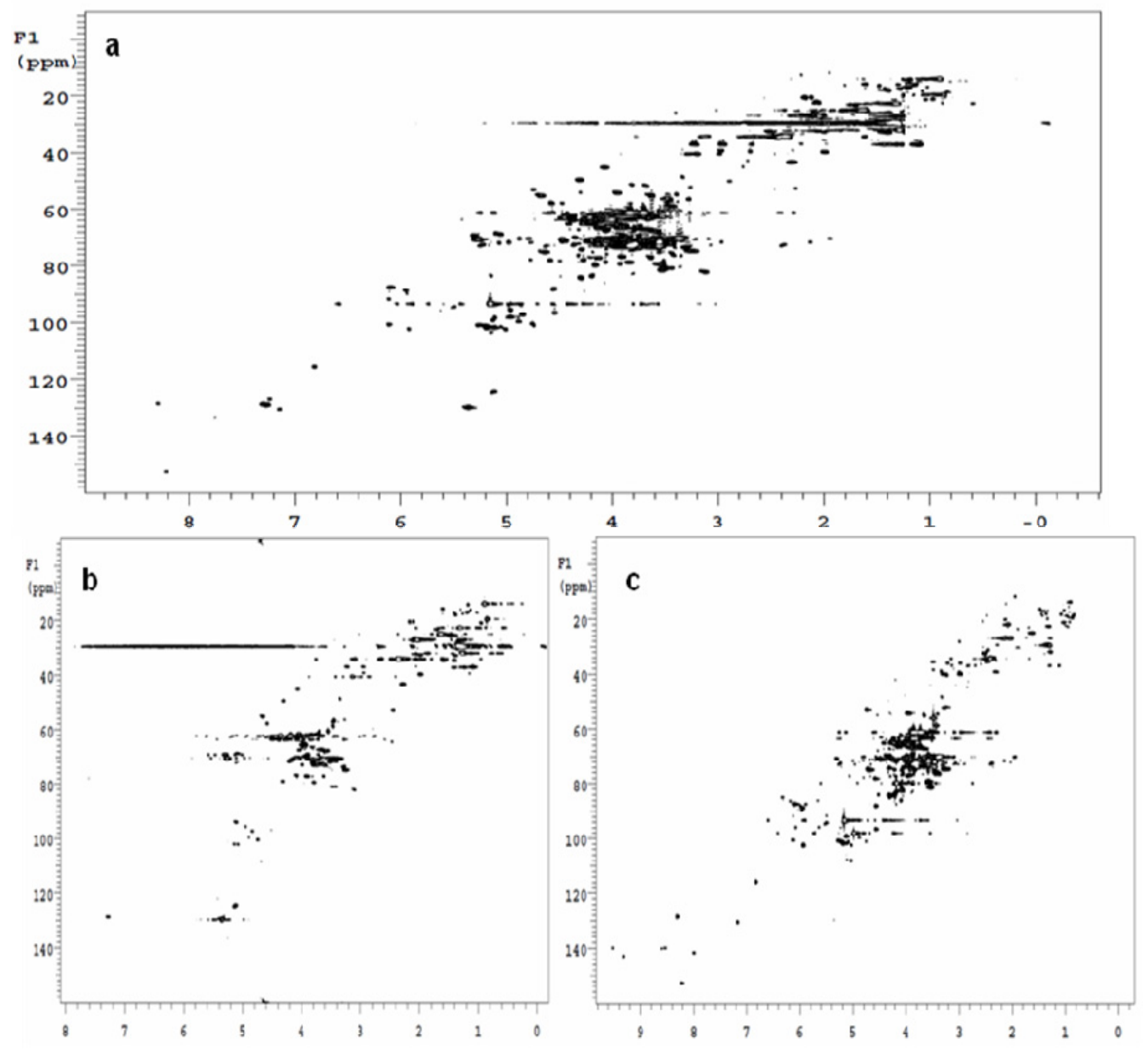

Figure A.12 Effect of Solvent Partition on the Lipid Profiles of M. smegmatis

Total lipid profile is shown in (a), apolar lipid profile is shown in (b), and the polar lipid profile is shown in (c). 
APPENDIX B. SUPPLEMENTARY FIGURES FOR CHAPTER 3

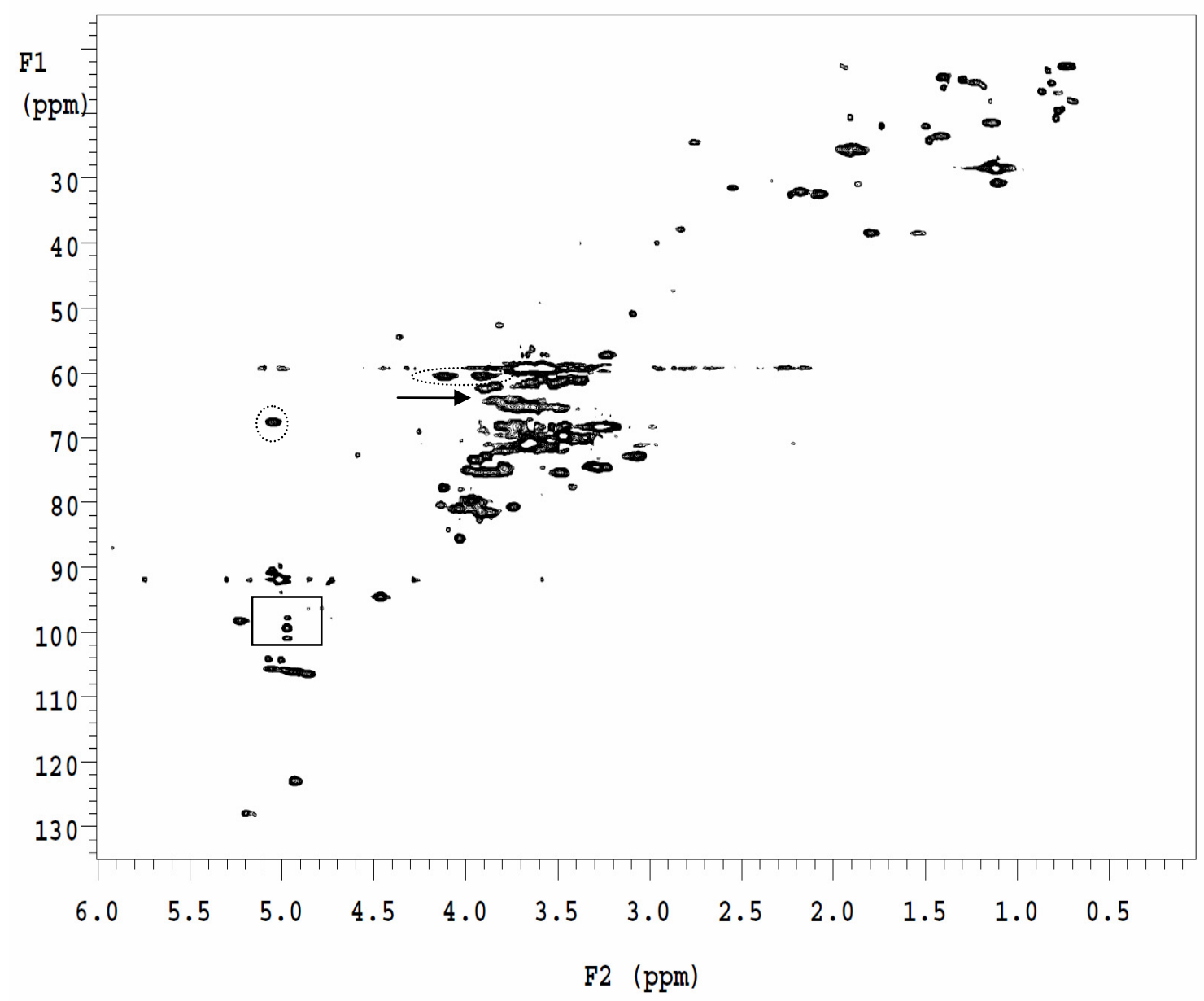

Figure B.1 HR-MAS 2D-HSQC Spectrum of Live M. abscessus

Highlighted in this figure is the presence of low LAM levels (enclosed in a solid-line box), the presence of phosphoinositol caps (very low intensity signal indicated by a black arrow) and the presence of high levels of TAG (enclosed in dashed-line circles). 


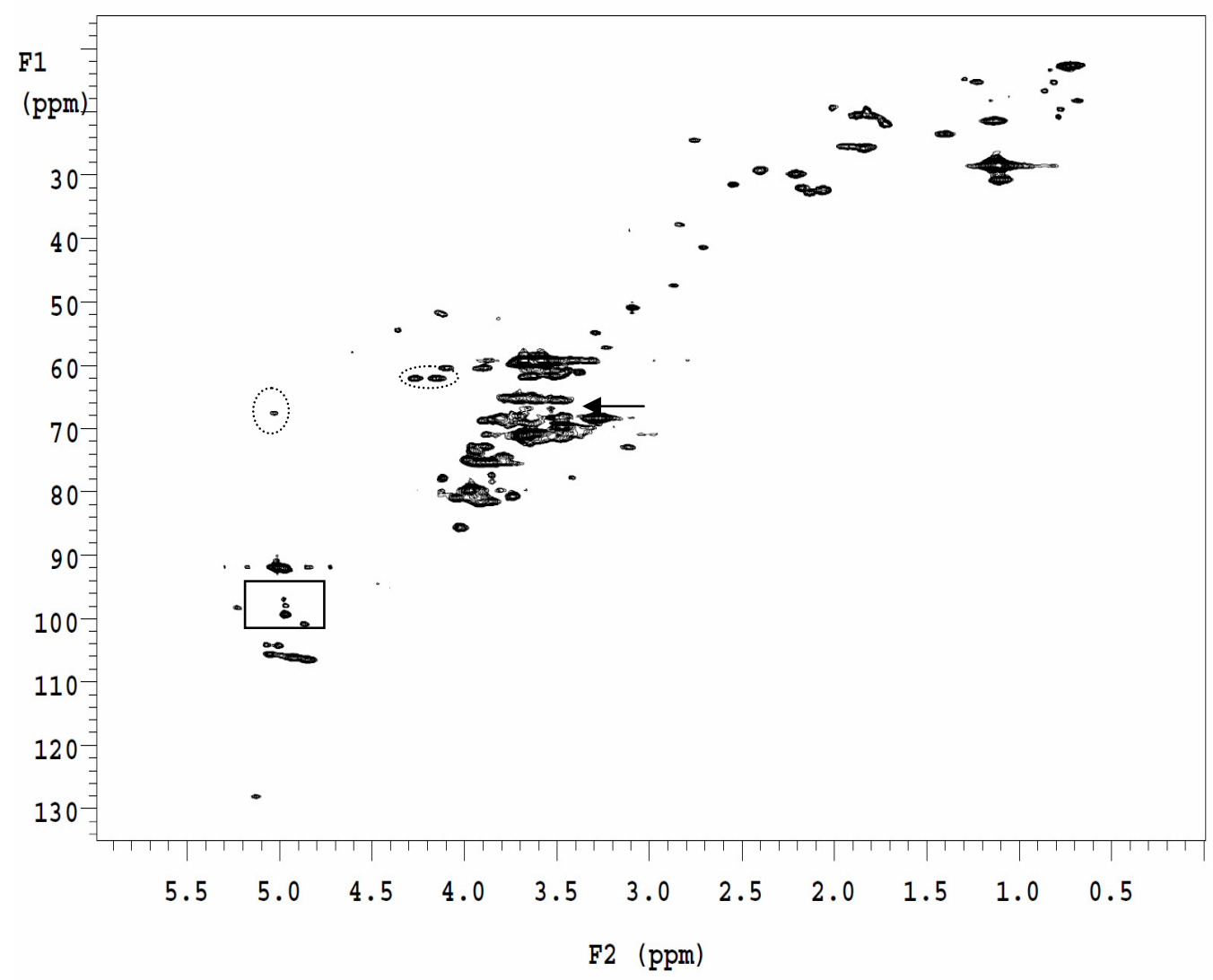

Figure B.2 HR-MAS 2D-HSQC Spectrum of Live M. avium

Highlighted in this figure is the presence of low LAM levels (enclosed in a solid-line box), the presence of mannose caps (indicated by a black arrow) and the presence of low levels of TAG (enclosed in dashed-line circles). 


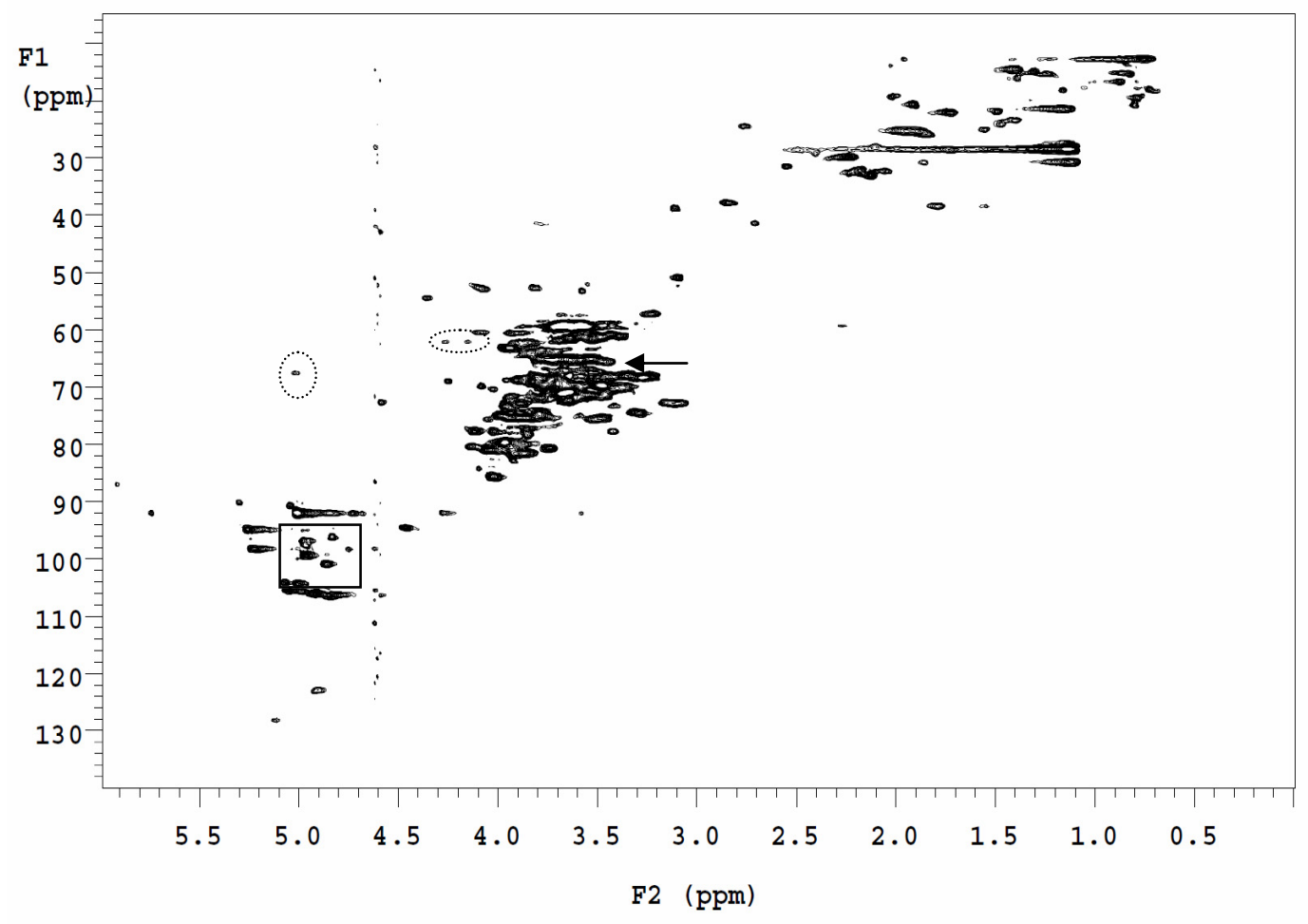

Figure B.3 HR-MAS 2D-HSQC Spectrum of Live M. bovis BCG

Highlighted in this figure is the presence of high LAM levels (enclosed in a solid-line box), the presence of mannose caps (indicated by a black arrow) and the presence of low levels of TAG (enclosed in dashed-line circles). 


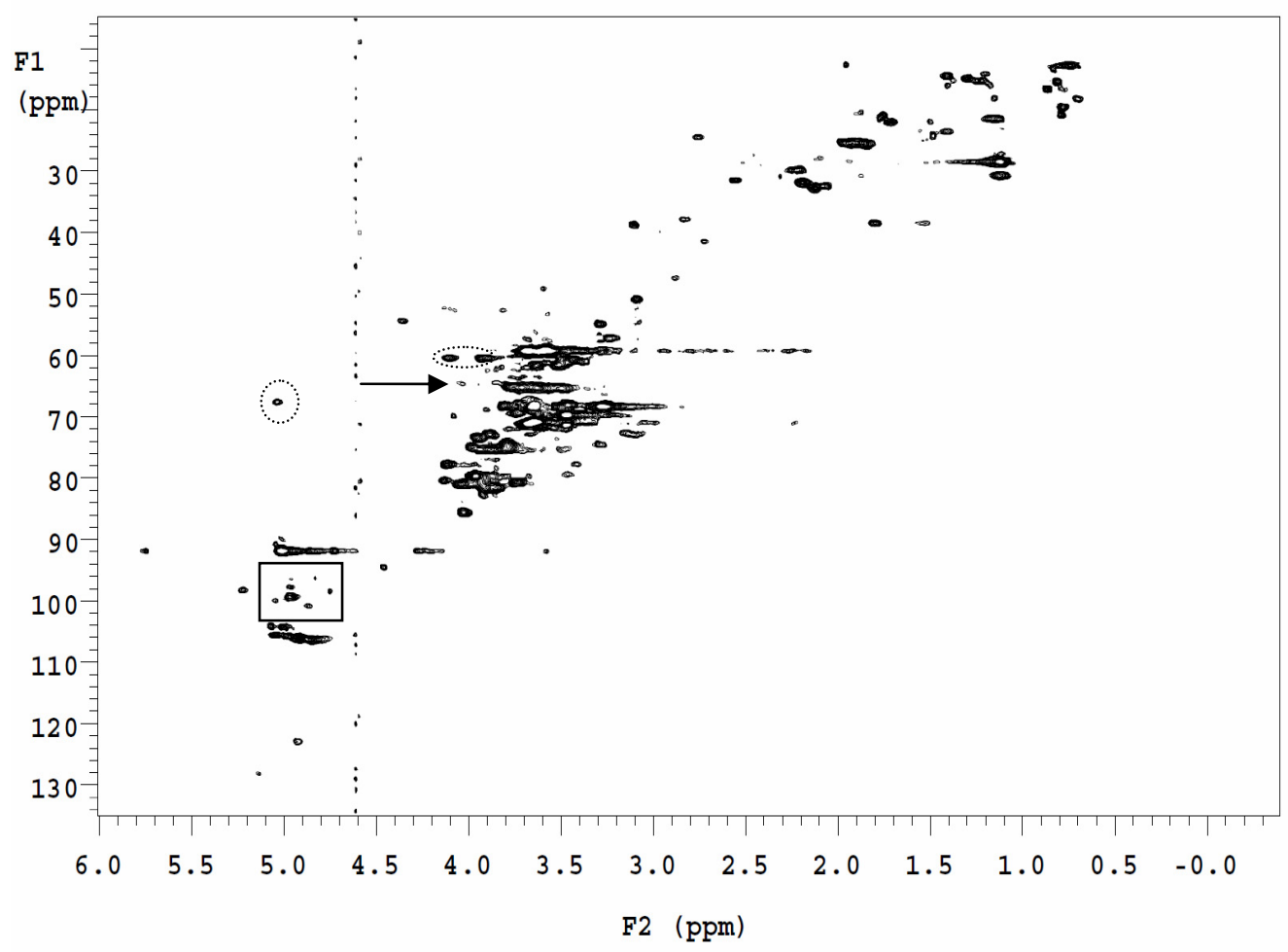

Figure B.4 HR-MAS 2D-HSQC Spectrum of Live M. fortitum

Highlighted in this figure is the presence of moderate LAM levels (enclosed in a solidline box), the presence of phosphoinositol caps (indicated by a black arrow) and the presence of high levels of TAG (enclosed in dashed-line circles). 


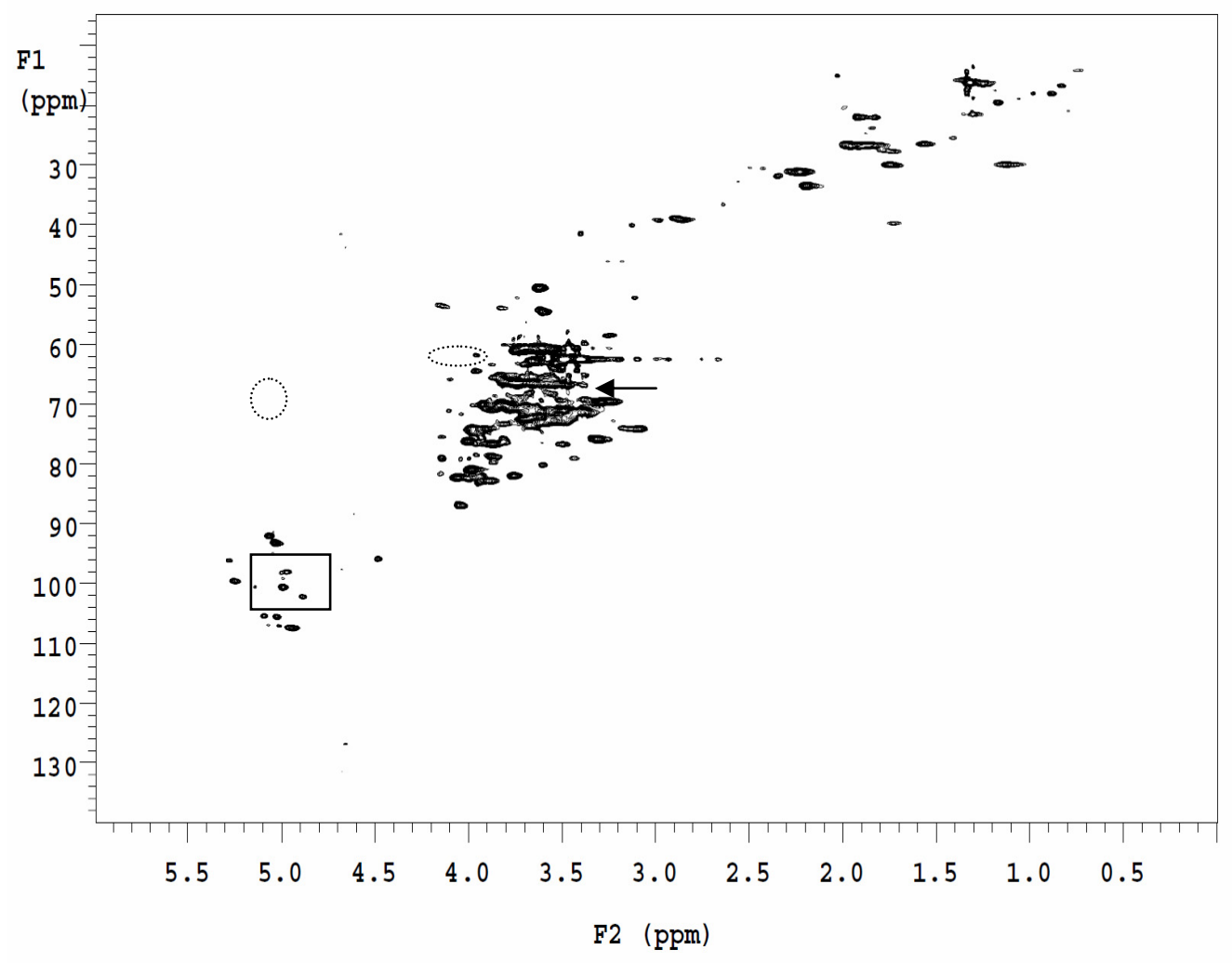

Figure B.5 HR-MAS 2D-HSQC Spectrum of Live M. liflandii

Highlighted in this figure is the presence of moderate LAM levels (enclosed in a solidline box), the presence of mannose caps (indicated by a black arrow) and the presence of low levels of TAG (very weak signal enclosed in dashed-line circles). 


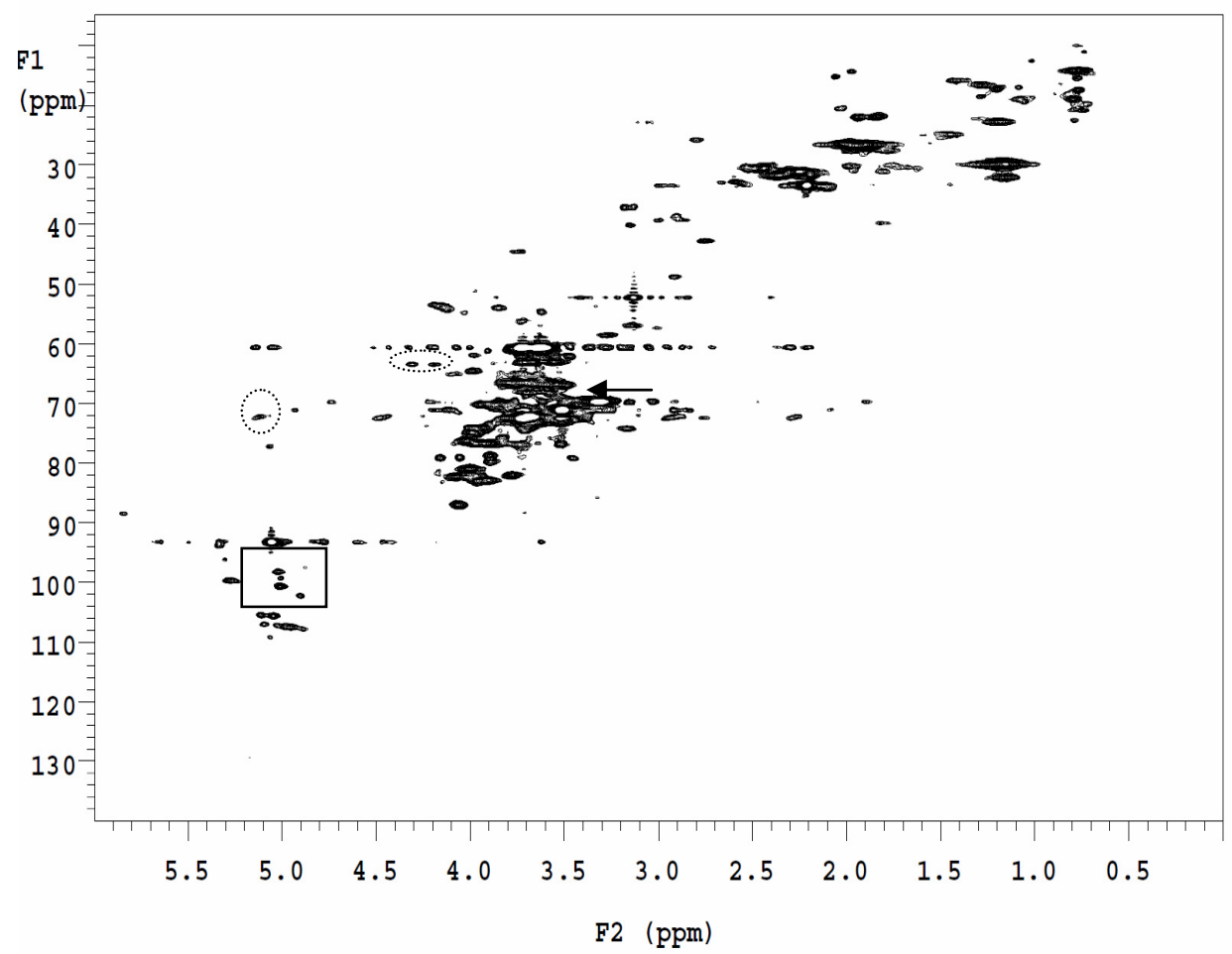

Figure B.6 HR-MAS 2D-HSQC Spectrum of Live M. marinum

Highlighted in this figure is the presence of moderate LAM levels (enclosed in a solidline box), the presence of mannose caps (indicated by a black arrow) and the presence of low levels of TAG (enclosed in dashed-line circles). 


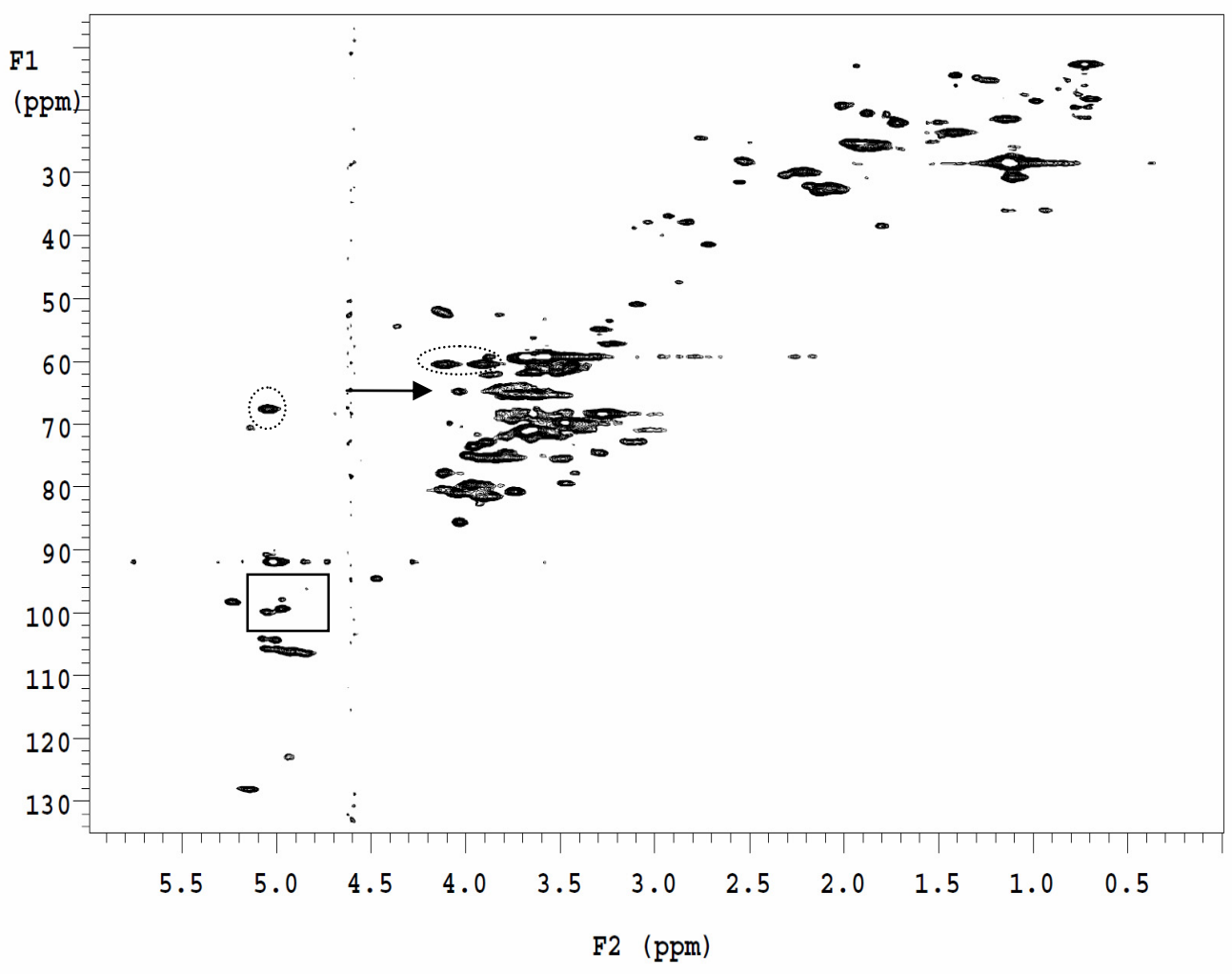

Figure B.7 HR-MAS 2D-HSQC Spectrum of Live M. phlei

Highlighted in this figure is the presence of low LAM levels (enclosed in a solid-line box), the presence of phosphoinositol caps (indicated by a black arrow) and the presence of high levels of TAG (enclosed in dashed-line circles). 


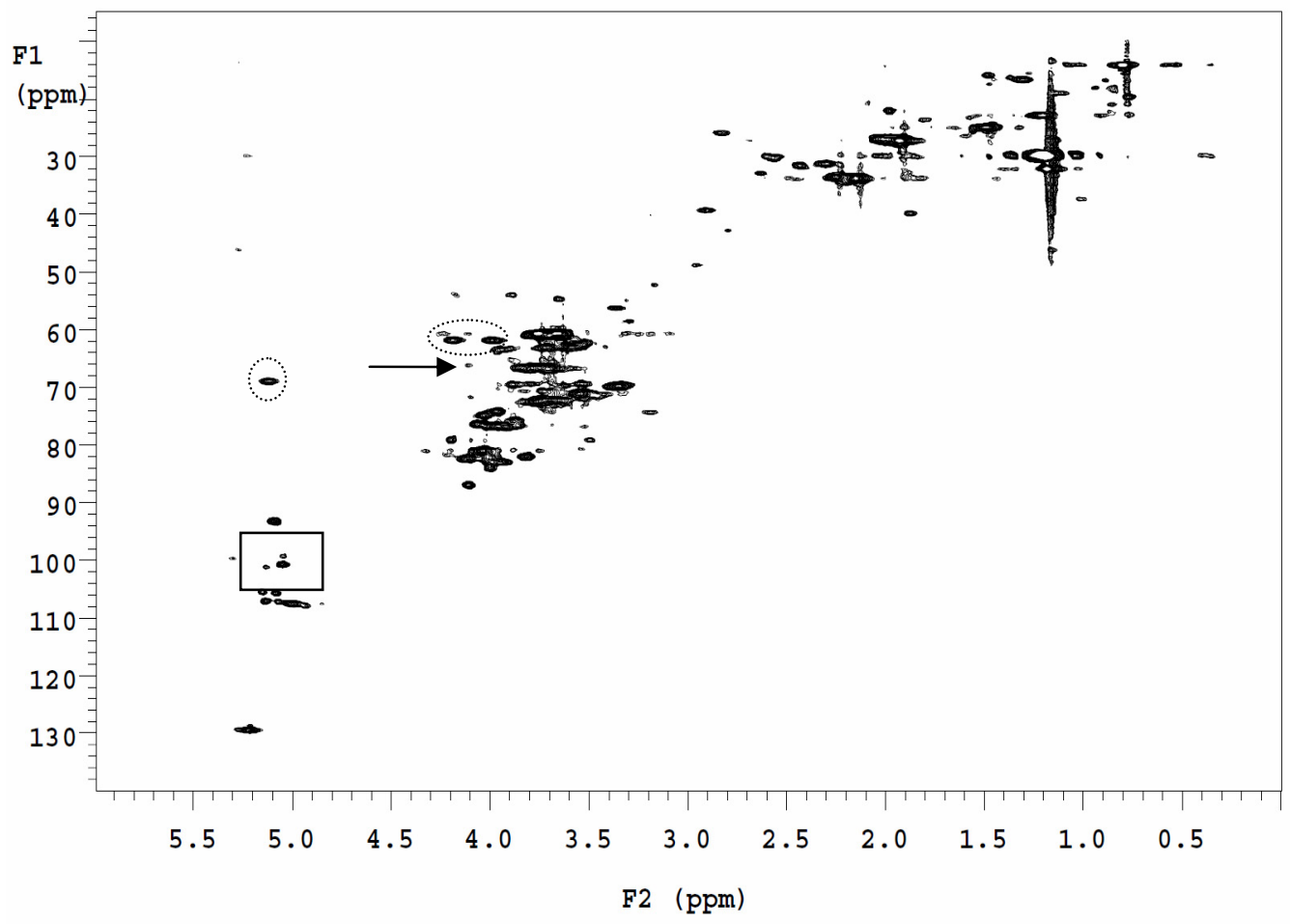

Figure B.8 HR-MAS 2D-HSQC Spectrum of Live M. smegmatis

Highlighted in this figure is the presence of low LAM levels (enclosed in a solid-line box), the presence of phosphoinositol caps (indicated by a black arrow) and the presence of high levels of TAG (enclosed in dashed-line circles). 


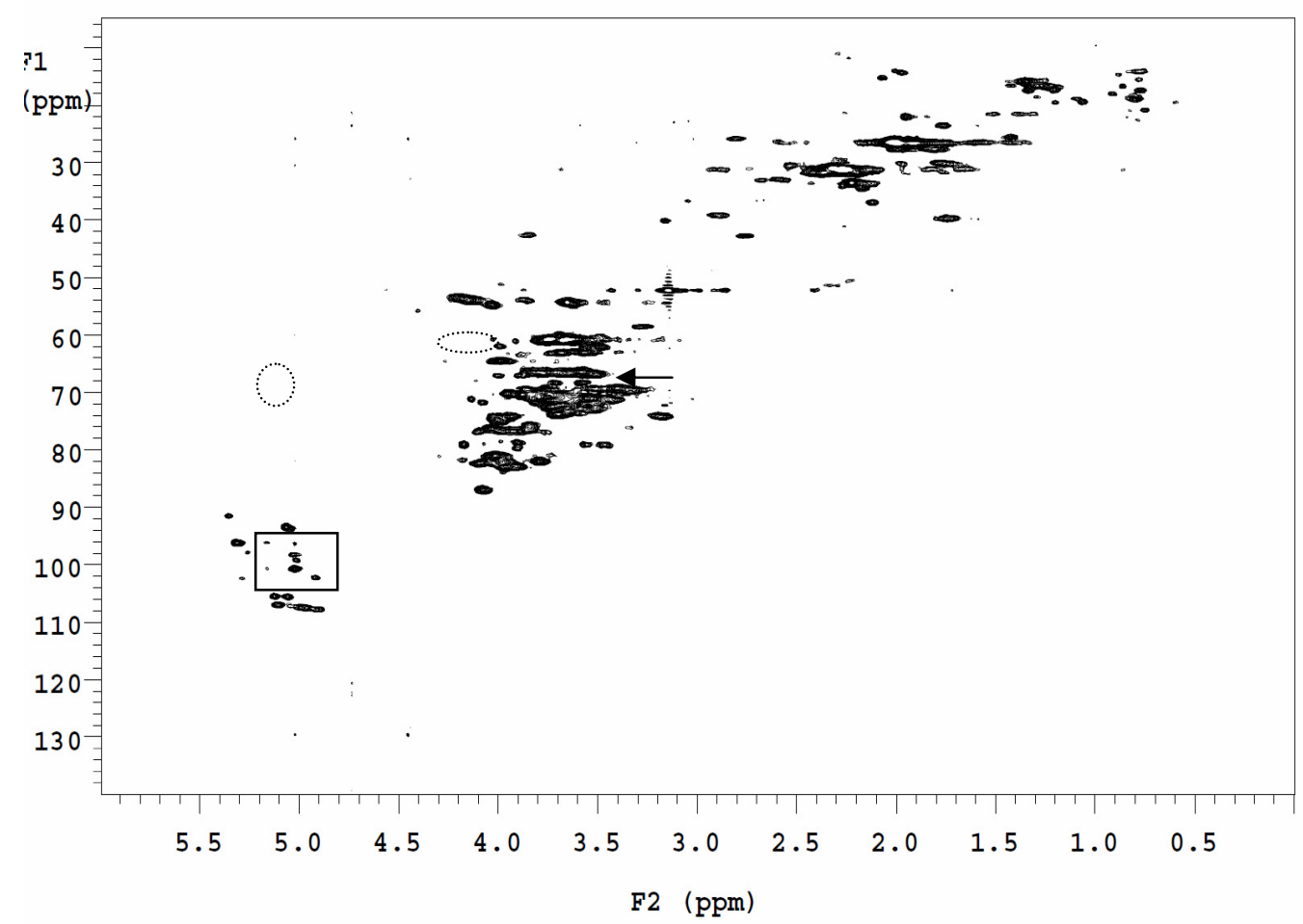

Figure B.9 HR-MAS 2D-HSQC Spectrum of Live M. tuberculosis $\mathrm{H}_{37} \mathrm{Ra}$

Highlighted in this figure is the presence of high LAM levels (enclosed in a solid-line box), the presence of mannose caps (indicated by a black arrow) and the absence of TAG (enclosed in dashed-line circles). 


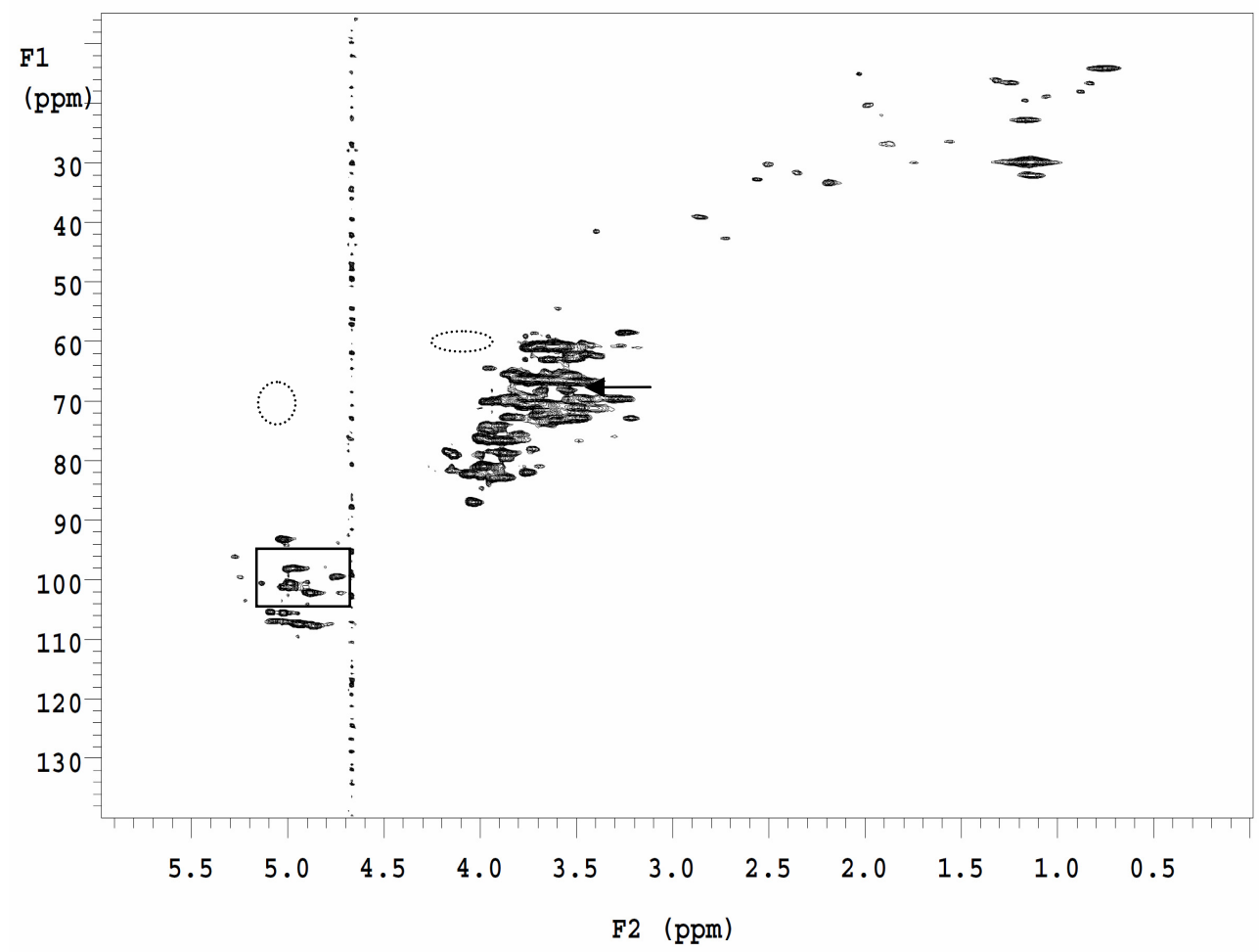

Figure B.10 HR-MAS 2D-HSQC Spectrum of Live M. ulcerans

Highlighted in this figure is the presence of high LAM levels (enclosed in a solid-line box), the presence of mannose caps (indicated by a black arrow) and the absence of TAG (enclosed in dashed-line circles). 


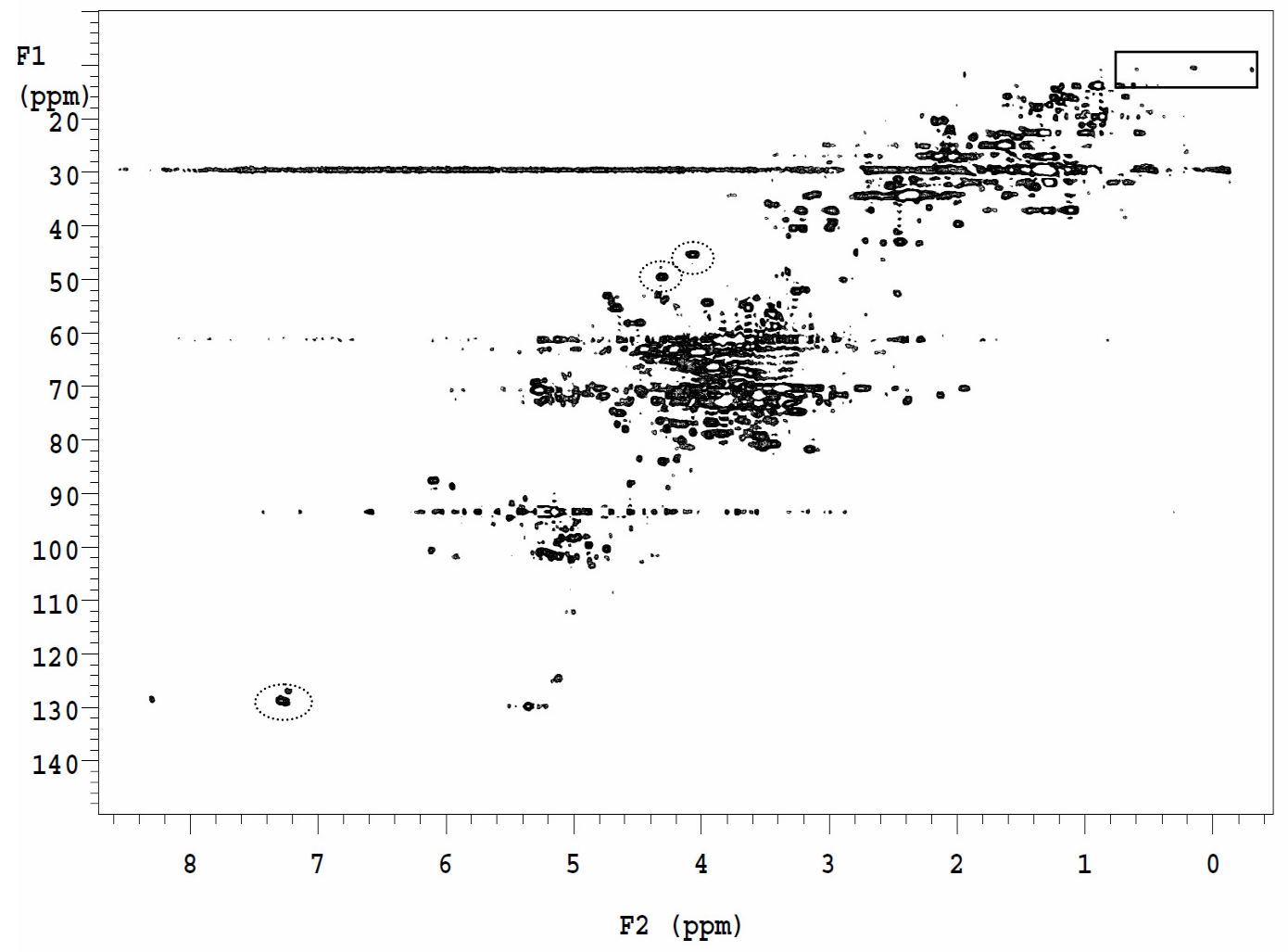

Figure B.11 2D-HSQC Lipid Profile of M. avium

Highlighted in the figure are signals of cyclopropyl mycolates (enclosed in a rectangular box) and signals of the GPL core (enclosed in dashed-line circles). 


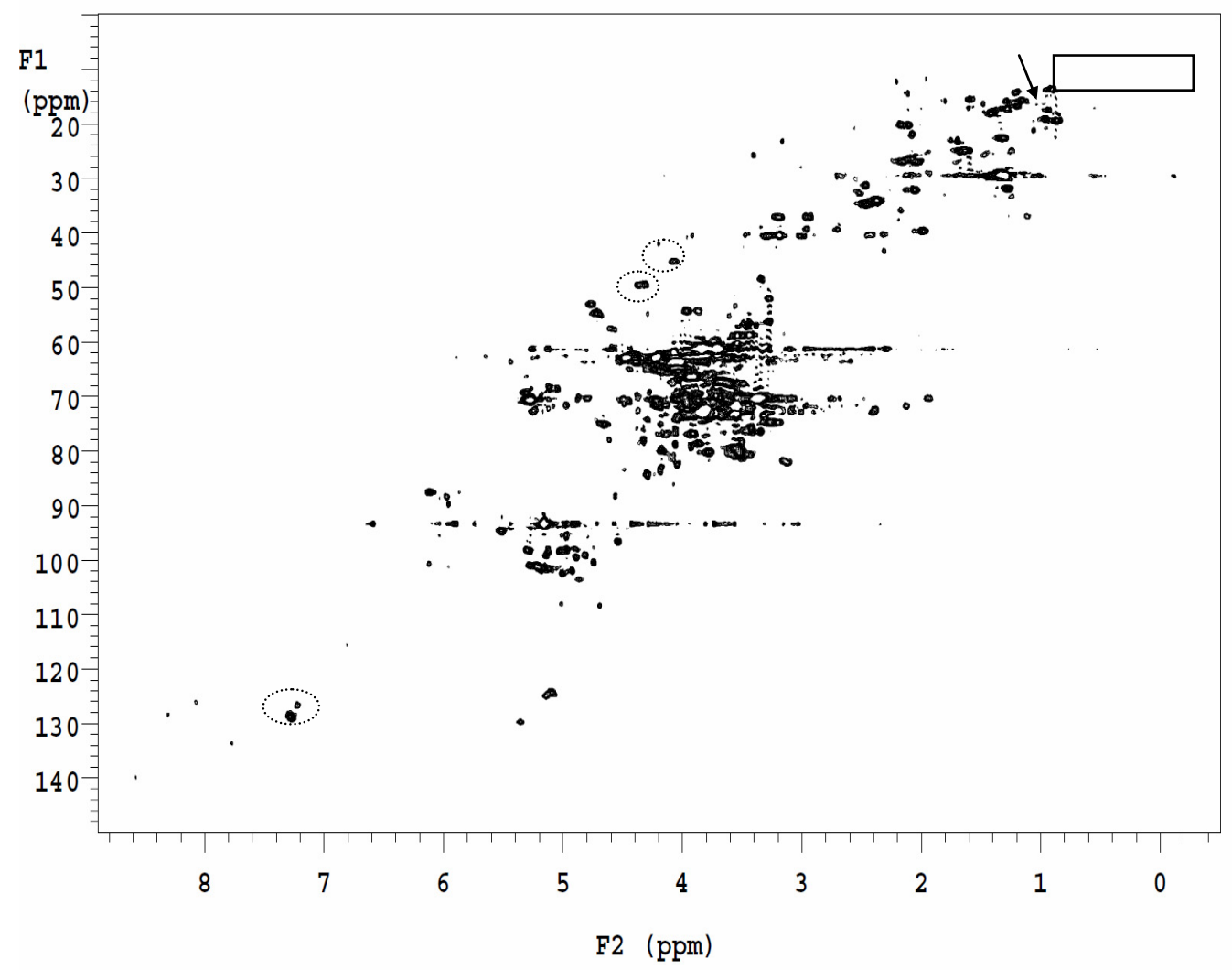

Figure B.12 2D-HSQC Lipid Profile of M. abcessus

Highlighted in the figure are signals from the GPL core, enclosed in dashed-line circles. Also noticeable, is the absence of cyclopropyl signals (as indicated by the rectangular box) and the presence of low intensity signals of methyl-branched fatty acids, indicated by a small arrow. 


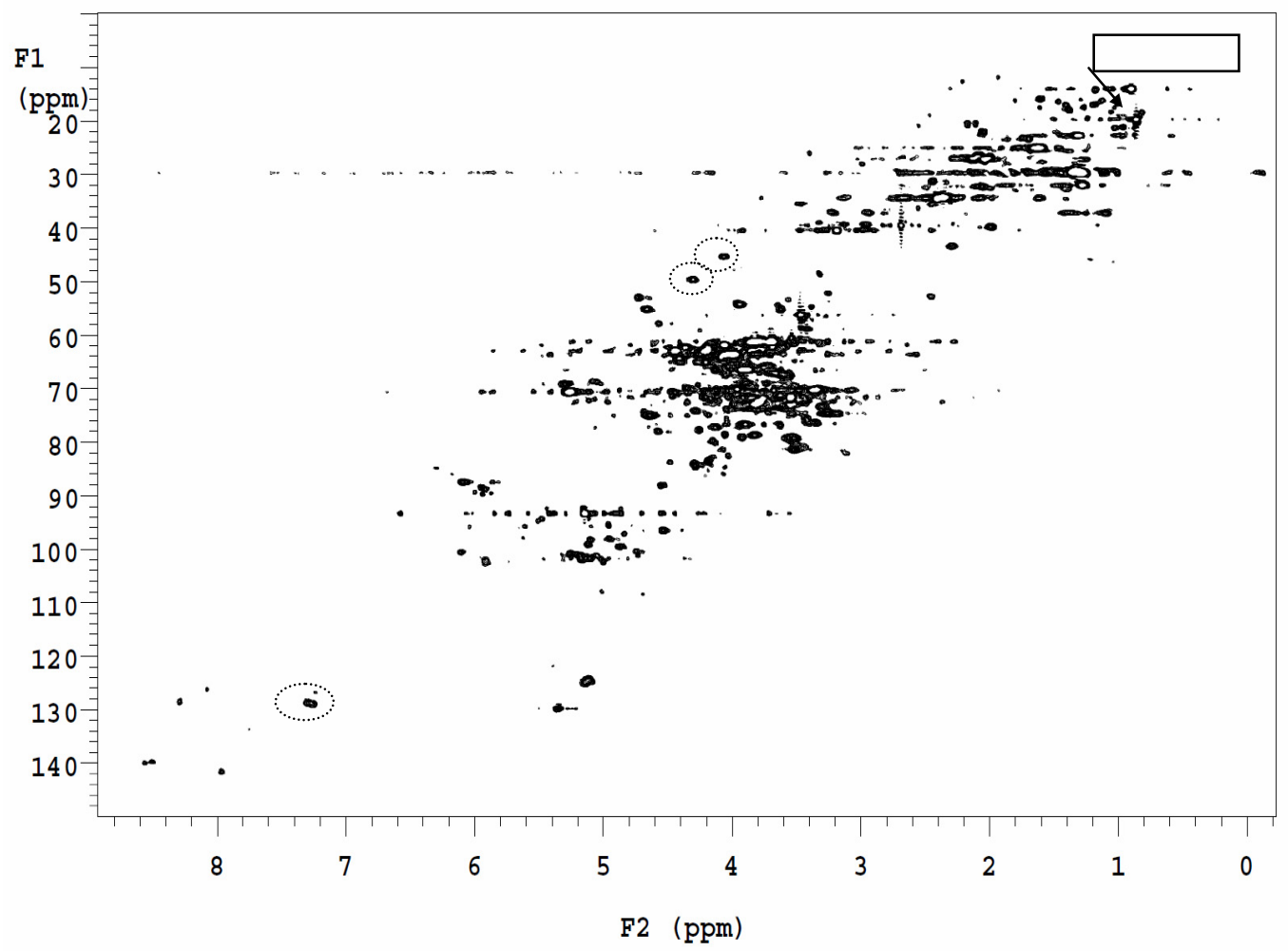

Figure B.13 2D-HSQC Lipid Profile of M. smegmatis

Highlighted in the figure are signals from the GPL core, enclosed in dashed-line circles. Also noticeable is the absence of cyclopropyl signals (as indicated by the rectangular box) and the absence of signals indicative of methyl-branched fatty acids, indicated by a small arrow. 


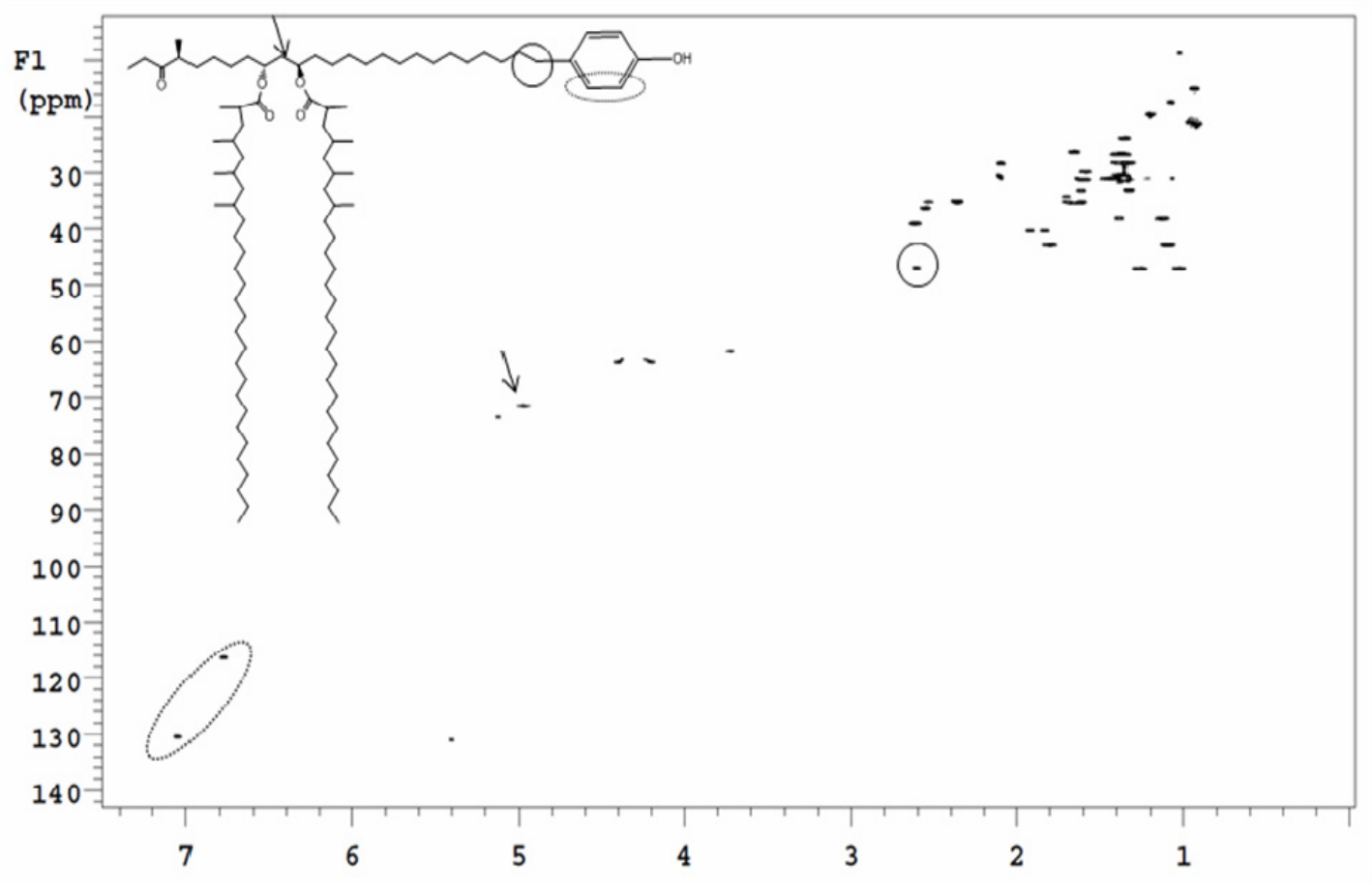

Figure B.14 2D-HSQC of Purified Phenol Phthiocerol Isolated from M. ulcerans

Highlighted in the 2D-HSQC spectrum and in the corresponding phenol phthiocerol are selected biomarker signals from different parts of the molecule. 


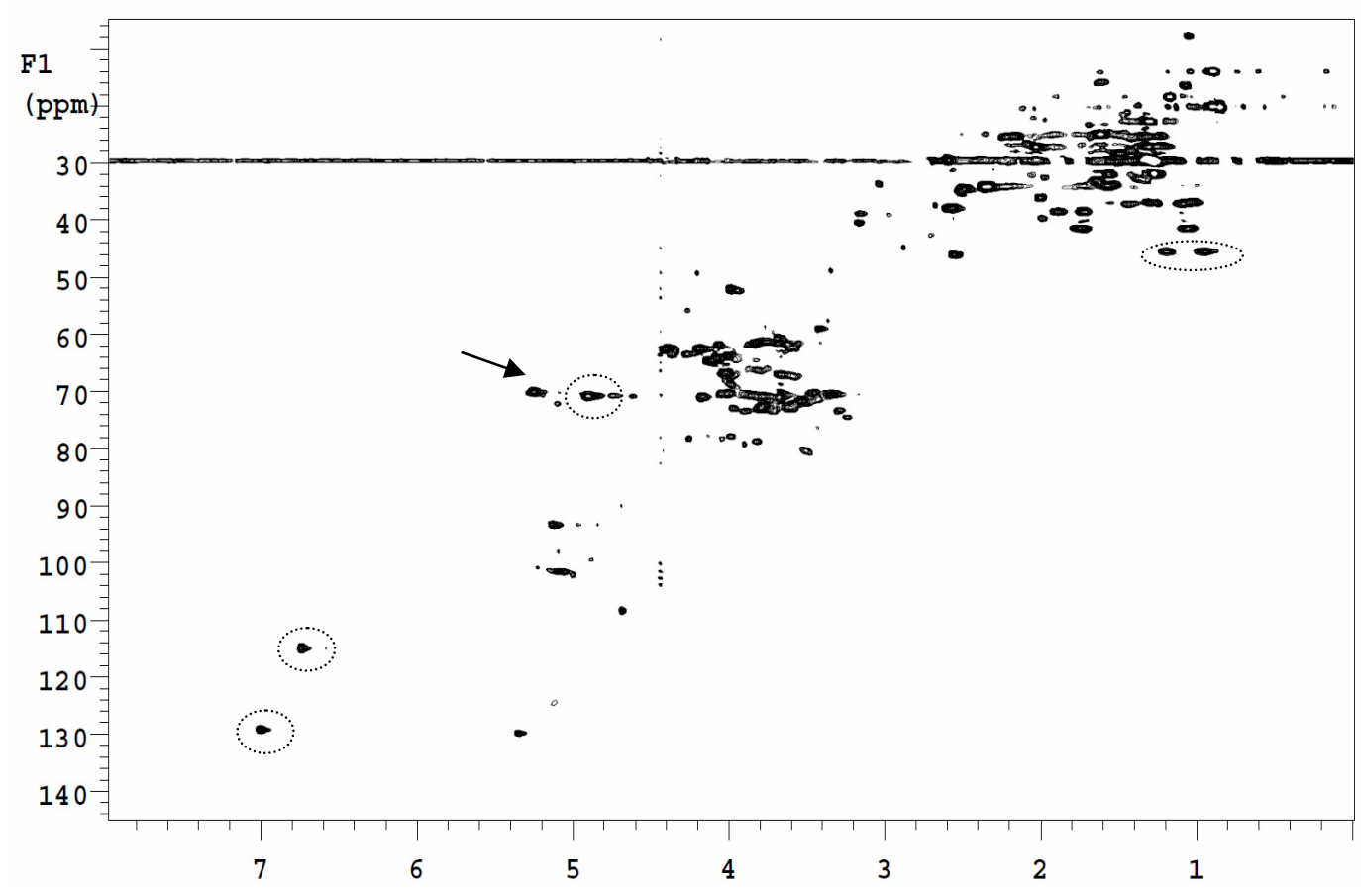

Figure B.15 2D-HSQC Lipid Profile of M. ulcerans

Highlighted in the figure are signals of phenol phthiocerol (enclosed in dashed-line circles). The absence of TAG signal from the lipid profile is indicated by a small arrow. 


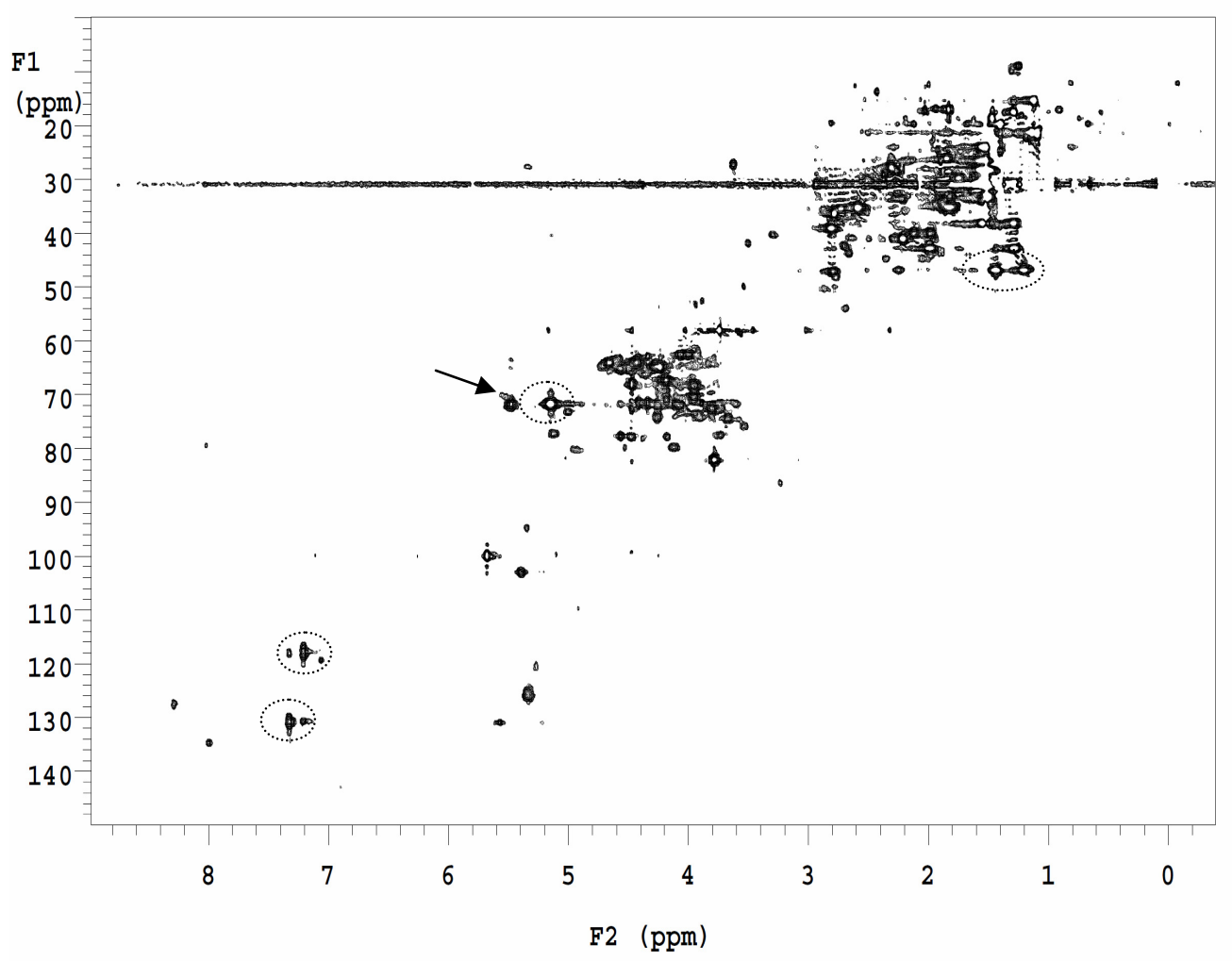

Figure B.16 2D-HSQC Lipid Profile of M. liflandii

Highlighted in the figure are signals of phenol phthiocerol and its corresponding PGL (enclosed in dashed-line circles, the aromatic signals of PGL are recorded at significantly higher intensities). The presence of low amount of TAG in the lipid profile is indicated by a small arrow. 


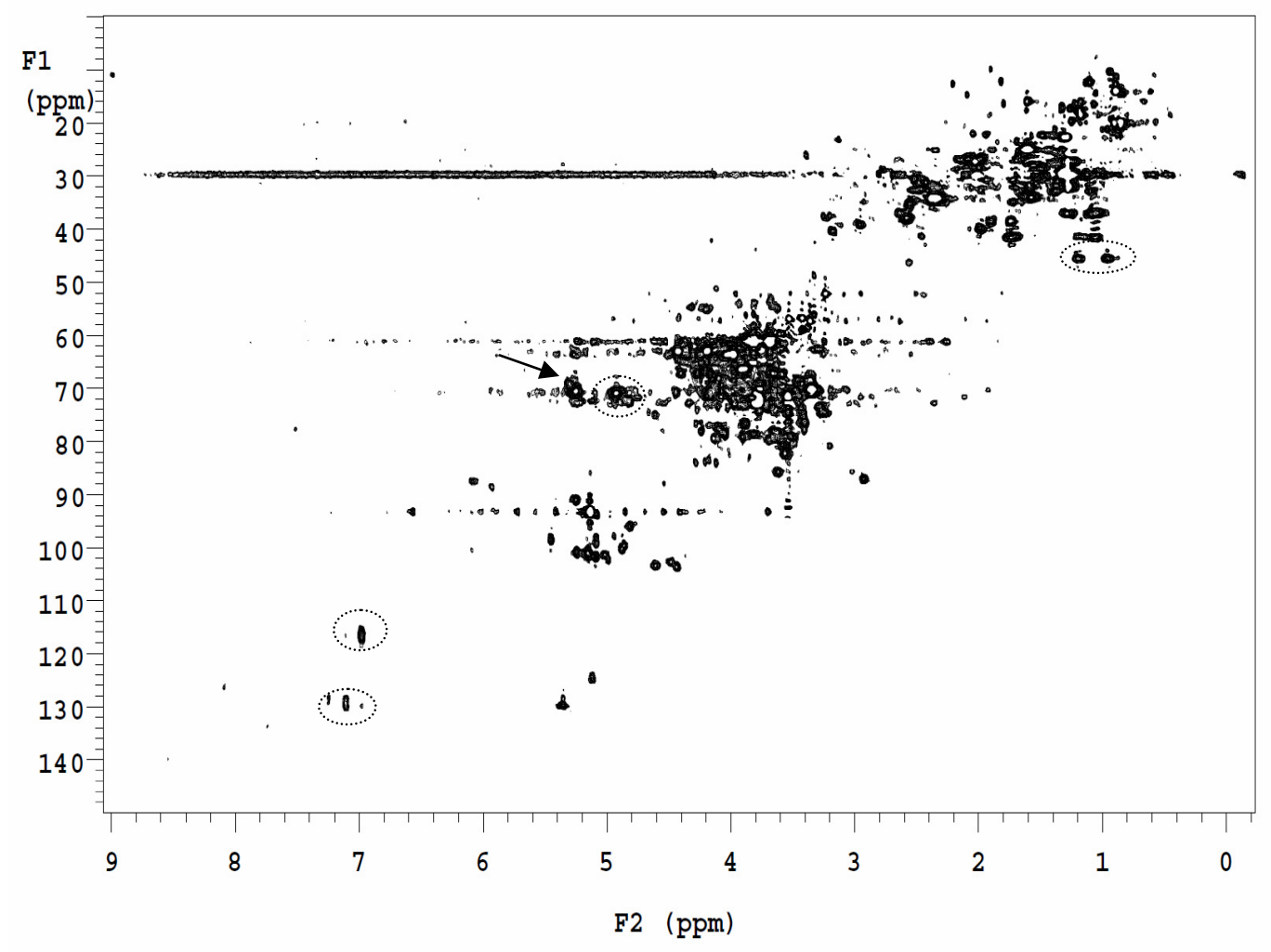

Figure B.17 2D-HSQC Lipid Profile of M. marinum

Highlighted in the figure are signals of phenol phthiocerol and its corresponding PGL (enclosed in dashed-line circles, the aromatic signals of PGL are recorded at significantly higher intensities). The presence of high levels of TAG in the lipid profile is indicated by a small arrow. 


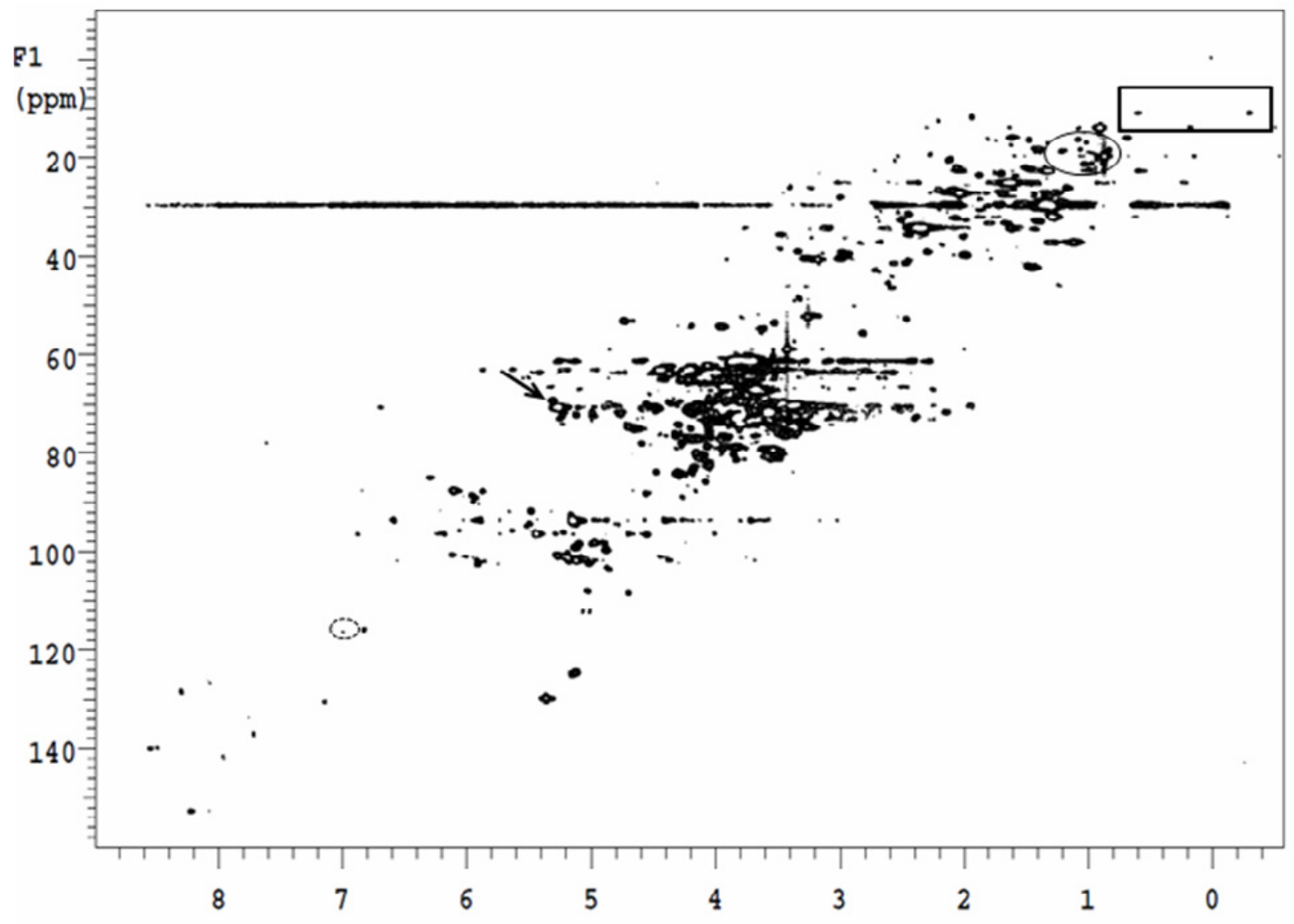

Figure B.18 2D-HSQC Lipid Profile of M. bovis $B C G$

Highlighted in the figure are signals indicative for cyclopropyl mycolates (in solid-line rectangle), methyl-branched fatty acids (in solid line circles), Mycoside B (the aromatic signal in dashed-line circles) and TAG (indicated by a black arrow). 


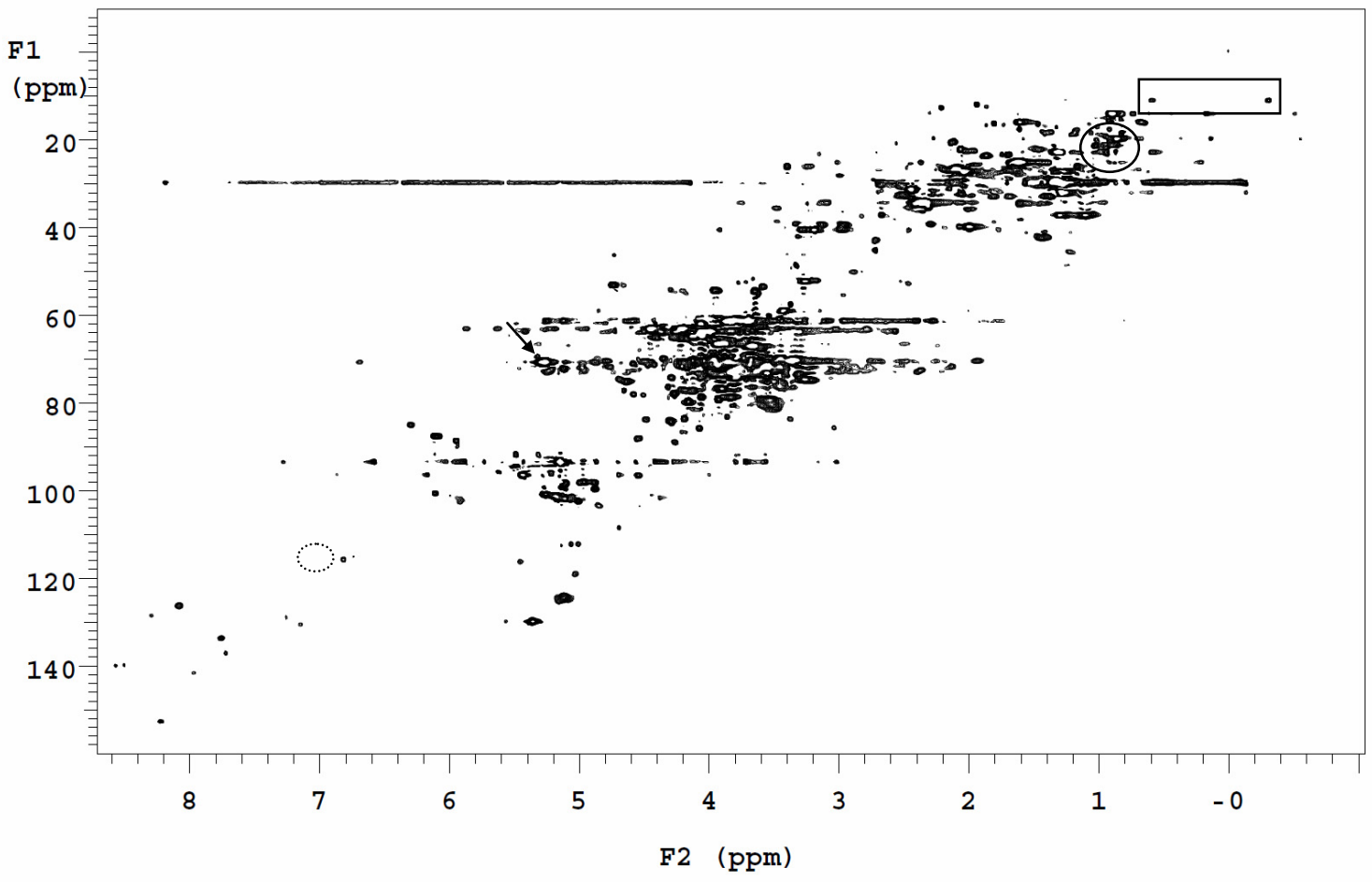

Figure B.19 2D-HSQC Lipid Profile of M. tubrerculosis $\mathrm{H}_{37} \mathrm{Rv}$

Highlighted in the figure are signals indicative for cyclopropyl mycolates (in solid-line rectangle), highly abundant methyl-branched fatty acids (in solid line circles), and low levels of TAG (indicated by a black arrow). The absence of mycoside B is indicated by the absence of its aromatic signal (dashed-line circle). 


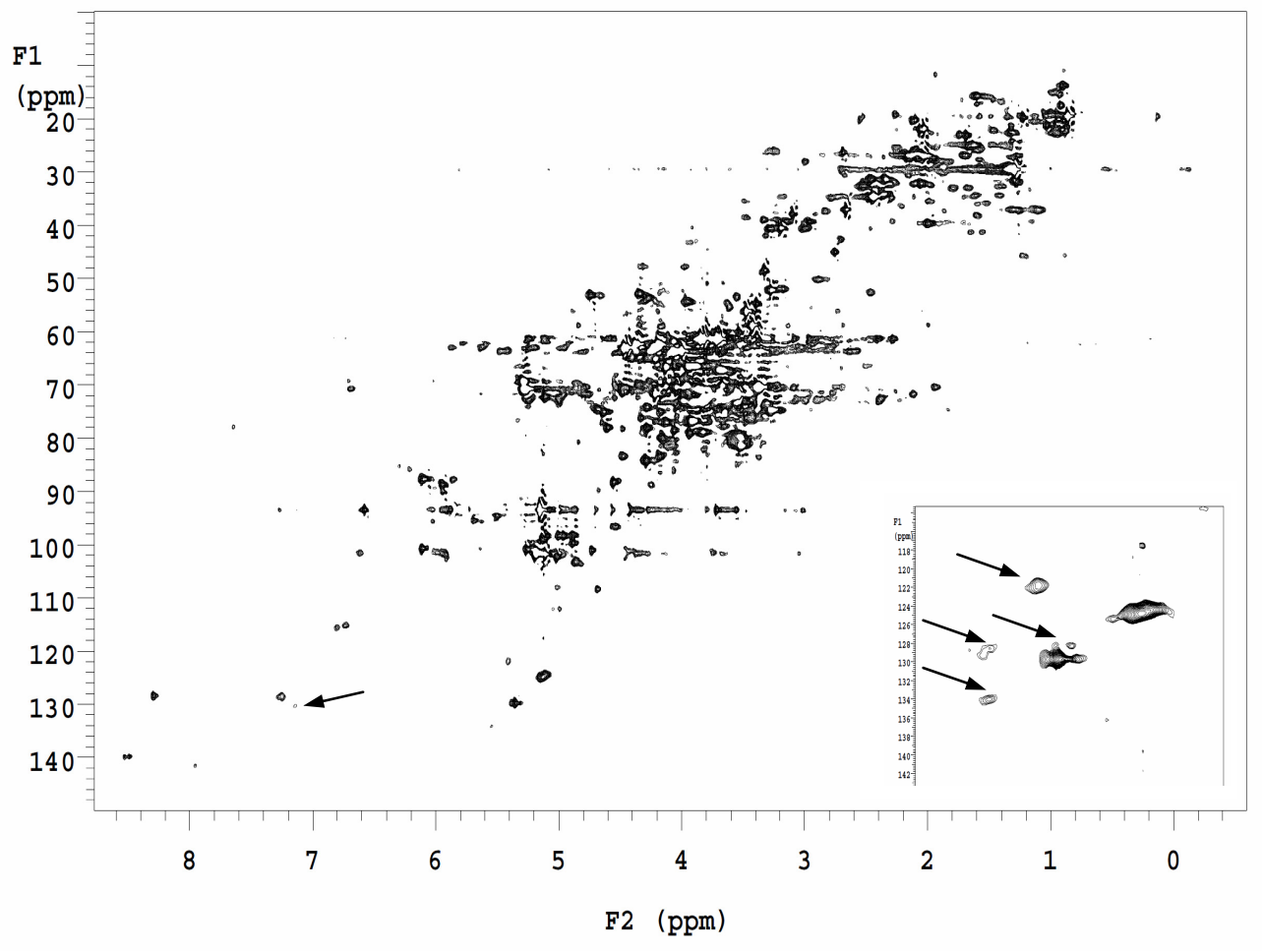

Figure B.20 2D-HSQC Lipid Profile of M. phlei

The bottom insert show the area where signals for trehalose phleiates can be observed. Phleic acid is a polyunsatured fatty acid. 


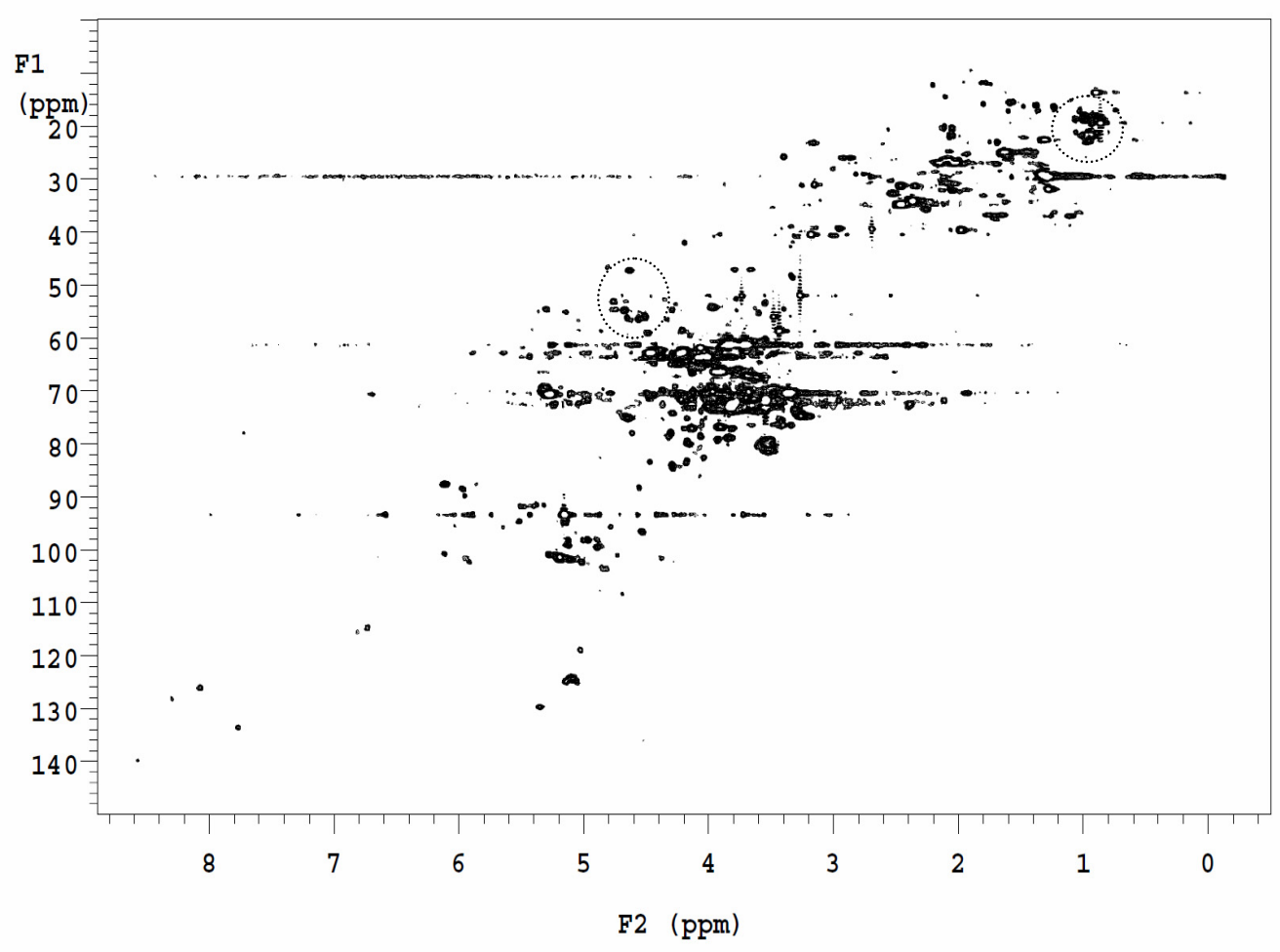

Figure B.21 2D-HSQC Lipid Profile of M. fortitum

Highlighted in the figure are signals specific to M. fortitum that are likely to be associated with its unique glycolipid, Mycolactone F. 


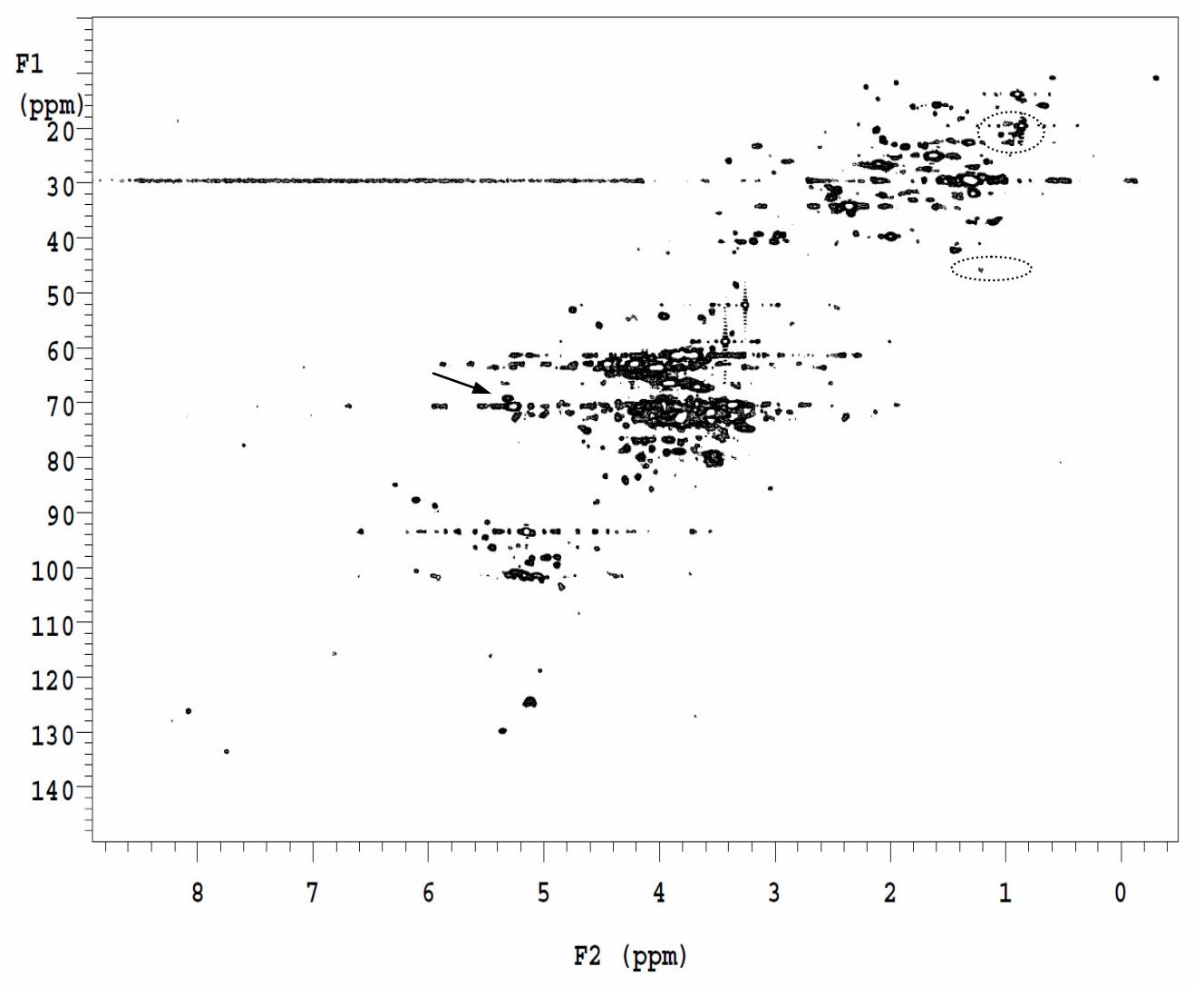

Figure B.22 2D-HSQC Lipid Profile of M. tuberculosis H37 Ra

Highlighted in the figure are signals indicative of methyl-branched fatty acids (reported at relatively low intensity and highlighted in dashed-line circles), and low levels of TAG (indicated by a black arrow). 


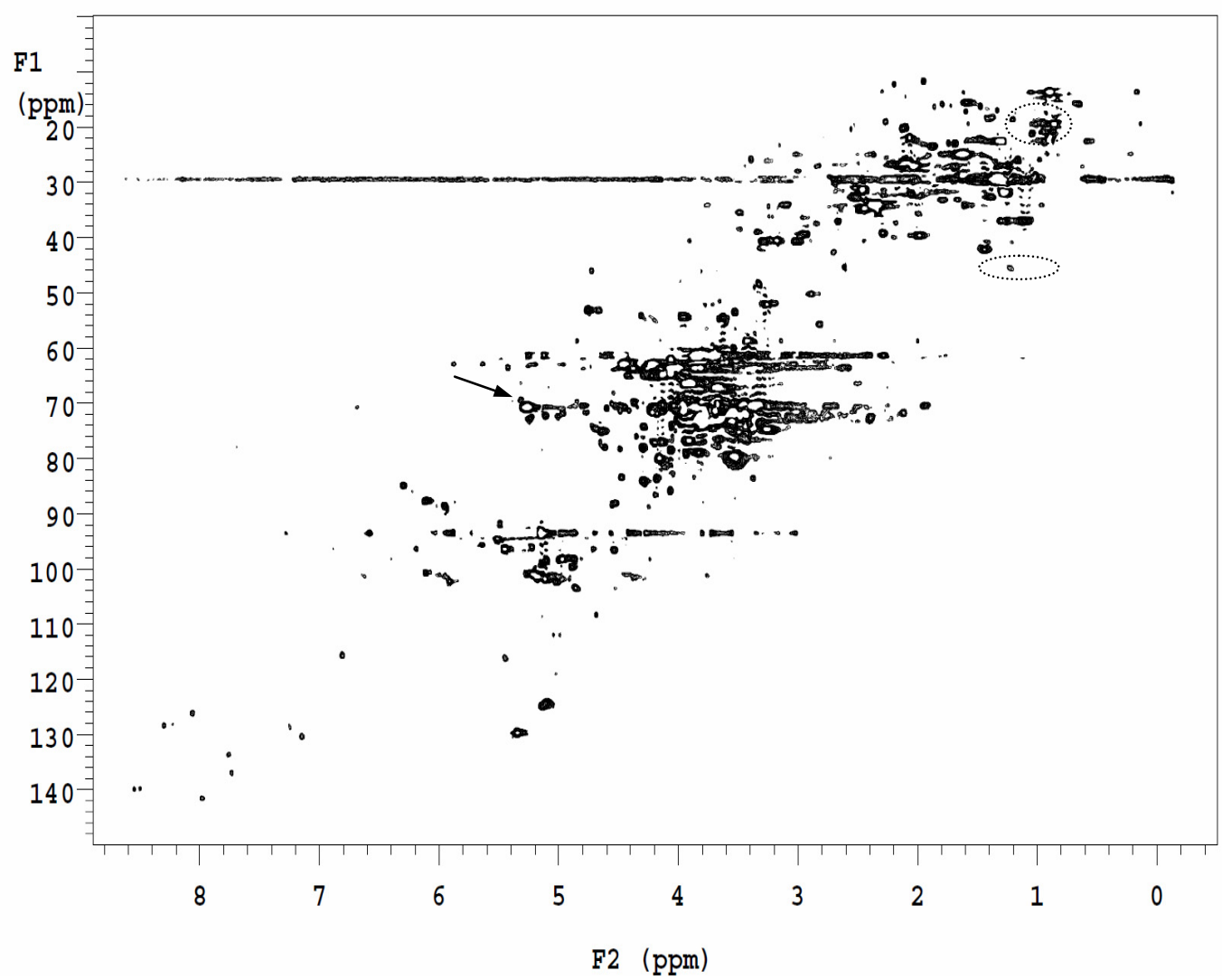

Figure B.23 2D-HSQC Lipid Profile of M. tuberculosis Erdman's Strain

Highlighted in the figure are signals indicative of methyl-branched fatty acids (reported at relatively higher intensity and highlighted in dashed-line circles), and low levels of TAG (indicated by a black arrow). 


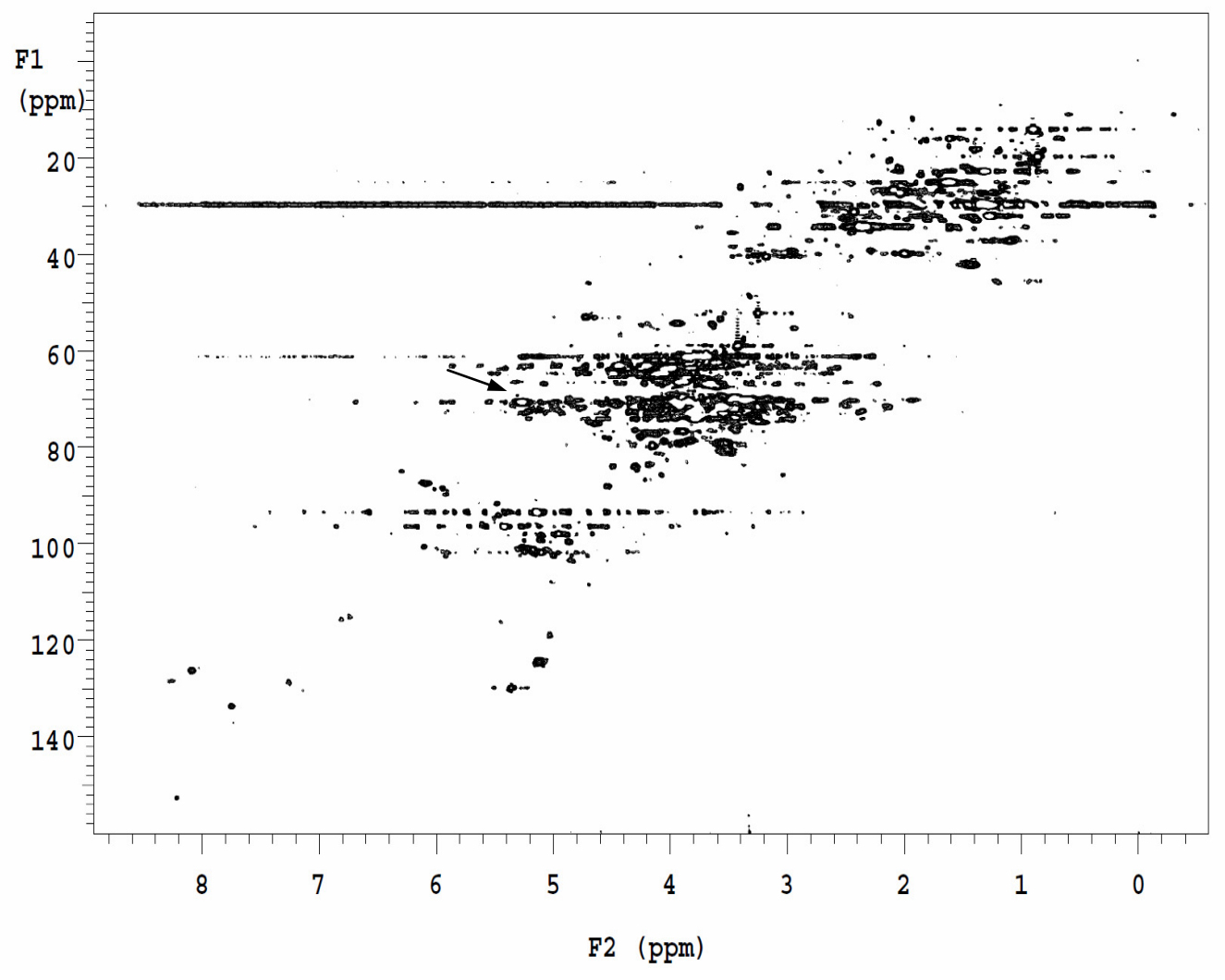

Figure B.24 2D-HSQC Lipid Profile of M. tuberculosis CDC1551 Strain

The presence of very low TAG levels is highlighted by a black arrow. 


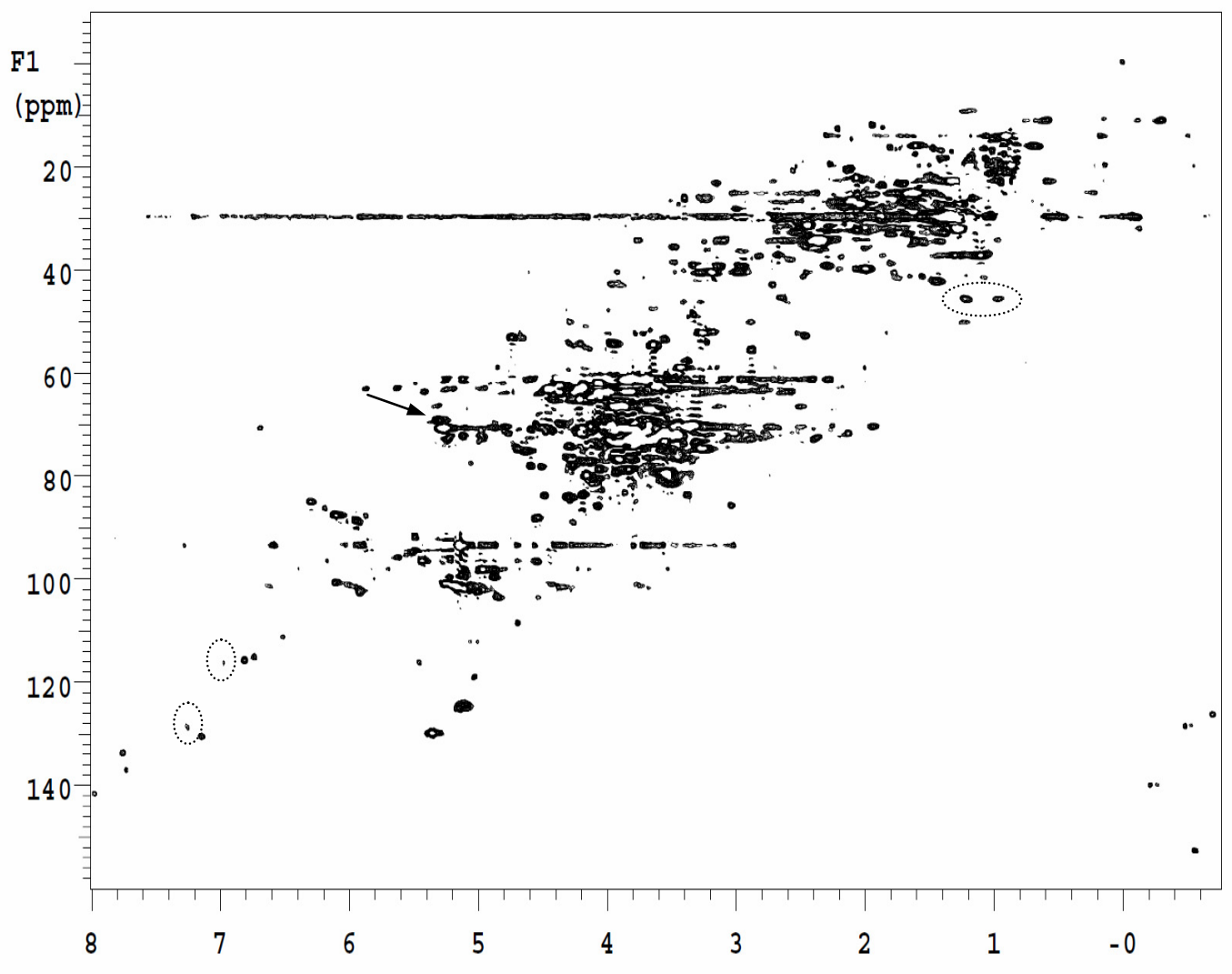

Figure B.25 2D-HSQC Lipid Profile of M. tuberculosis HN878 Strain

Highlighted in the figure, are signals indicative for M. tuberculosis PGL (signals for PGL are enclosed in dashed-line circles). Also highlighted by a black arrow are relatively higher levels of TAG. 


\section{VITA}

Engy Abdelhameed Mahrous was born in Giza, Egypt on June 6, 1977. She obtained her Bachelor degree in Pharmacy and Pharmaceutical Sciences from the College of Pharmacy, Cairo University in 1999. After graduation, she was appointed as a laboratory instructor in the department of pharmacognosy in the same college. She started working as a research assistant in the department of Veteran Affairs in Memphis in February of 2004 under the supervision of Dr. Malak Kotb. In August 2004, she joined the graduate program of pharmaceutical sciences in the University of Tennessee Health Science Center and joined the research group of Dr. Richard E. Lee. 